\title{
Dietary (Poly)phenolics in Human Health: Structures, Bioavailability, and Evidence of Protective Effects Against Chronic Diseases
}

\author{
Daniele Del Rio,, Ana Rodriguez-Mateos, ${ }^{2}$ Jeremy P.E. Spencer, ${ }^{2}$ \\ Massimiliano Tognolini, ${ }^{3}$ Gina Borges, ${ }^{4}$ and Alan Crozier ${ }^{4}$
}

\begin{abstract}
Human intervention trials have provided evidence for protective effects of various (poly)phenol-rich foods against chronic disease, including cardiovascular disease, neurodegeneration, and cancer. While there are considerable data suggesting benefits of (poly)phenol intake, conclusions regarding their preventive potential remain unresolved due to several limitations in existing studies. Bioactivity investigations using cell lines have made an extensive use of both (poly)phenolic aglycones and sugar conjugates, these being the typical forms that exist in planta, at concentrations in the low- $\mu M$-to-mM range. However, after ingestion, dietary (poly)phenolics appear in the circulatory system not as the parent compounds, but as phase II metabolites, and their presence in plasma after dietary intake rarely exceeds $\mathrm{nM}$ concentrations. Substantial quantities of both the parent compounds and their metabolites pass to the colon where they are degraded by the action of the local microbiota, giving rise principally to small phenolic acid and aromatic catabolites that are absorbed into the circulatory system. This comprehensive review describes the different groups of compounds that have been reported to be involved in human nutrition, their fate in the body as they pass through the gastrointestinal tract and are absorbed into the circulatory system, the evidence of their impact on human chronic diseases, and the possible mechanisms of action through which (poly)phenol metabolites and catabolites may exert these protective actions. It is concluded that better performed in vivo intervention and in vitro mechanistic studies are needed to fully understand how these molecules interact with human physiological and pathological processes. Antioxid. Redox Signal. 18, 1818-1892.
\end{abstract}

I. Introduction

II. Classification of Phenolic Compounds

A. Flavonoids

1. Flavonols

2. Flavones

3. Isoflavones

4. Flavanones

5. Anthocyanidins

6. Flavan-3-ols

B. Nonflavonoids

Reviewing Editors: Jeffrey Blumberg, Evan P. Cherniack, Jose M. Estrela, Isaac Ginsburg, Liudmila Korkina, Hasan Mukhtar, Etsuo Niki, Patricia Oteiza, Sampath Parthasarathy, Saveria Pastore, Marzia Perluigi, and Francesco Visioli

\footnotetext{
${ }^{1}$ The Laboratory of Phytochemicals in Physiology, Human Nutrition Unit, Department of Food Science, University of Parma, Parma, Italy.

${ }^{2}$ Molecular Nutrition Group, Department of Food and Nutritional Sciences, School of Chemistry, Food and Pharmacy, University of Reading, Reading, United Kingdom.

${ }^{3}$ Department of Pharmacy, University of Parma, Parma, Italy.

${ }^{4}$ Plant Products and Human Nutrition Group, School of Medicine, University of Glasgow, Glasgow, United Kingdom.
} 
III. Bioavailability of Flavonoids and Related Compounds 1825

A. Flavonols and flavanones 1828

B. Anthocyanins 1830

C. Flavones

D. Isoflavones 1834

E. Flavan-3-ols

1. Cocoa flavan-3-ol monomers 1838

2. Green tea flavan-3-ol monomers 1838

$\begin{array}{lr}\text { a. Dose effects } & 1839\end{array}$

b. Colonic catabolism of green tea flavan-3-ols $\quad 1840$

c. Identification and quantification of flavan-3-ol metabolites 1840

3. Black tea theaflavins and thearubigins 1842

F. Proanthocyanidins 1843

G. Dihydrochalcones 1843

H. Ellagitannins 1845

I. Chlorogenic acids

$\begin{array}{ll}\text { J. Resveratrol } & 1848\end{array}$

K. Plant lignans 1849

IV. Evidence for the Accumulation of (Poly)phenol Metabolites in Body Tissues 1849

V. In Vitro Biological Activity and Mode of Action 1851

A. Anthocyanins 1851

B. Chlorogenic acids 1852

C. Ellagitannins 1853

D. Flavan-3-ols

E. Flavonols

F. Lignans and isoflavones 1858

G. Flavanones 1859

VI. Feeding Studies and Evidence of Protective Effects 1859

A. Cardiovascular effects of dietary polyphenols 1859

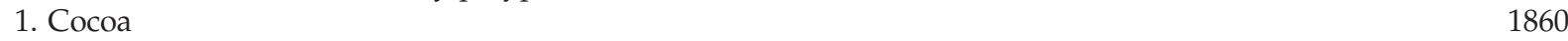

2. Red wine 1861

3. Tea 1868

$\begin{array}{ll}\text { 4. Berries } & 1868\end{array}$

5. Pomegranate 1869

$\begin{array}{ll}\text { 6. Citrus fruit } & 1870\end{array}$

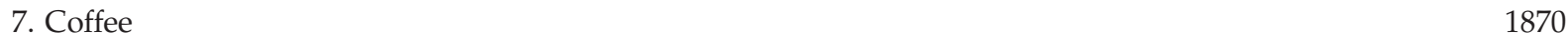

8. Nuts 1870

B. (Poly)phenols and neurodegenerative diseases 1870

C. (Poly)phenols and cancer 1871

VII. Conclusions 1872

\section{Introduction}

W HILE NOT ESSENTIAL for the successful growth and development of most plants, flavonoids and related phenolic compounds* can occur in high concentrations in some species and are referred to as secondary metabolites. They are structurally diverse with in excess of 8000 structures having been reported (447), and many are found in only a limited number of species. In planta, they have various functions, including protecting plants from herbivores and microbial infection, as attractants for pollinators and seeddispersing animals, as allelopathic agents, UV protectants, and signal molecules in the formation of nitrogen-fixing root nodules $(95,218)$.

*Flavonoids and related compounds are also referred to as polyphenols. However, a number of compounds, including hydroxycinnamates and phenolic acids, have only one phenolic ring. In this review, therefore, the term (poly)phenolic compounds will be used.
The role of flavonoids and related compounds, as components responsible, in part, for the protective effects of a fruitand vegetable-rich diet has become an increasingly important area of human nutrition research. Unlike the traditional vitamins, they are not essential for short-term well being, but there is increasing evidence that modest long-term intakes can have favorable effects on the incidence of cancers and chronic diseases, including cardiovascular disease (CVD), type II diabetes, and impaired cognitive function, which are occurring with increasing frequency in Western populations (433). This review will summarize the different groups of compounds that are involved, their fate in the body after ingestion as they pass through the gastrointestinal tract (GIT) and are absorbed into the circulatory system, the evidence of their protective impact on human health, and the possible mechanisms of action through which their metabolites may exert such effects.

\section{Classification of Phenolic Compounds}

Phenolic compounds have at least one aromatic ring with one or more hydroxyl groups attached and are classified as 
flavonoids and nonflavonoids. Processed foods and beverages, such as black tea, matured red wine, coffee, and cocoa, may contain phenolic transformation products that are best described as derived polyphenols (95).

\section{A. Flavonoids}

Flavonoids are polyphenolic compounds comprising 15 carbons with two aromatic rings connected by a three-carbon bridge (Fig. 1). The main subclasses of these $C_{6}-C_{3}-C_{6}$ compounds are the flavones, flavonols, flavan-3-ols, isoflavones, flavanones, and anthocyanidins. Other flavonoid groups that are more minor dietary components are the chalcones, dihydrochalcones, dihydroflavonols, flavan-3,4-diols, coumarins, and aurones. The basic flavonoid skeleton can have numerous substituents. The majority of flavonoids occur naturally as glycosides rather than aglycones.

1. Flavonols. Flavonols occur widely throughout the plant kingdom with the exception of fungi and algae. The most common flavonols, kaempferol, quercetin, isorhamnetin, and myricetin (Fig. 2), are typically found as glycosides with conjugation occurring at the $5,7,3^{\prime}, 4^{\prime}$, and $5^{\prime}$ positions. Although the number of aglycones is limited, there are more than 200 sugar conjugates of kaempferol (447). There is information on the flavonol content of commonly consumed fruits, vegetables, and beverages with sizable differences in the amounts found in seemingly similar produce, possibly due to local growing conditions, seasonal changes, and varietal differences $(94,194,195,298)$. Yellow and red onions (Allium cepa) are especially rich source of flavonols containing high concentrations of quercetin- $4^{\prime}$-O-glucoside and quercetin-3,4'-O-diglucoside. The disaccharide quercetin3-O-rutinoside (Fig. 2) is a common dietary component.

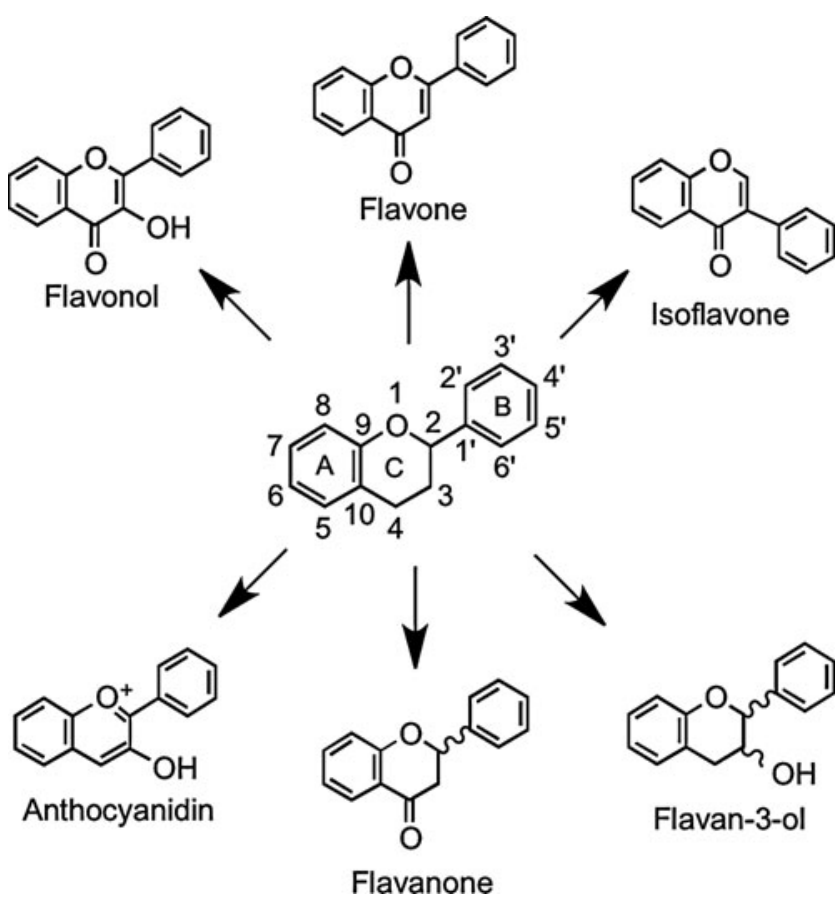

FIG. 1. Structure of the flavonoid skeleton.
2. Flavones. Flavones, such as apigenin, luteolin, wogonin, and baicalein, are similar structurally to flavonols, except they lack oxygenation at C-3 (Fig. 3). A wide range of substitutions is possible with flavones, including hydroxylation, methylation, $\mathrm{O}$ - and $\mathrm{C}$-glycosylation, and alkylation. In general, flavones are not distributed widely, although substantial amounts have been detected in celery (Apium graveolens), parsley (Petroselinum hortense), and some herbs. Many flavones occur as 7-O-glycosides, although rooibos tea, a caffeine-free beverage prepared from leaves of the South African shrub Aspalathus linearis, contains small amounts of apigenin-8-C-glucoside (vitexin), apigenin-6-C-glucoside (isovitexin), luteolin-8-C-glucoside (orientin), and luteolin-6C-glucoside (iso-orientin) $(80,437)$. Polymethoxylated flavones, such as nobiletin and tangeretin (Fig. 3), occur in citrus species.

3. Isoflavones. Isoflavones have the B-ring attached at C-3 rather than at the C-2 position (Fig. 1). They are found almost exclusively in leguminous plants with substantial quantities of daidzein and genistein occurring in soybean (Glycine max) principally as 7-O-(6"-O-malonyl)glucosides with lower amounts of the corresponding 7-O-(6"-Oacetyl)glucosides, 7-O-glucosides, and the aglycones (Fig. 4). Fermented soy products can be rich in the aglycones as a result of hydrolysis of the glycosides, whereas products whose manufacture involves heating, such as soy milk and tofu, contain reduced quantities of isoflavones, mainly in the form of the daidzein and genistein glucosides, which form as a result of degradation of malonyl- and acetylglucosides (88).

Because of their structural similarity to estrogen, isoflavones are classified as phytoestrogens, as are the nonflavonoid lignans, which are a diverse group of compounds that occur in high concentrations principally in cereal grains (Fig. 5).

4. Flavanones. Flavanones such as naringenin and hesperetin are characterized by the absence of $\Delta^{2,3}$ double bond and the presence of a chiral center at C-2 (Fig. 1). In planta flavanones occur predominantly as the $S$ - or (-)-enantiomer with the C-ring attached to the B-ring at C-2 in the $\alpha$ configuration (93). Flavanones occur as hydroxyl, glycosylated, and $\mathrm{O}$-methylated derivatives (Fig. 6). They are present in especially high amounts in flavedo of citrus fruits. The most common flavanone glycoside is hesperetin-7-O-rutinoside (hesperidin). Flavanone rutinosides are tasteless, in contrast to flavanone neohesperidoside conjugates, such as hesperetin7-O-neohesperidoside (neohesperidin) from bitter oranges (Citrus aurantium) and naringenin-7-O-neohesperidoside (naringin) from grapefruit (Citrus paradise), which have an intense bitter taste.

5. Anthocyanidins. The most common anthocyanidin aglycones are pelargonidin, cyanidin, delphinidin, peonidin, petunidin, and malvidin (Fig. 7), which form conjugates with sugars and organic acids to generate a multitude of anthocyanins of differing colors, ranging from orange and red to blue and purple, and as a consequence, they are readily visible in fruits and flowers $(219,338)$.

6. Flavan-3-ols. Flavan-3-ols, unusually, do not exist in planta predominantly as glycosides. They are the most 
<smiles>O=c1c(O)c(-c2ccc(O)cc2)oc2cc(O)cc(O)c12</smiles>

Kaempferol<smiles>COc1cc(-c2oc3cc(O)cc(O)c3c(=O)c2O)ccc1O</smiles>

Isorhamnetin<smiles></smiles>

Quercetin-4'-O-glucoside (spiroeoside)<smiles>O=c1c(O)c(-c2ccc(O)c(O)c2)oc2cc(O)cc(O)c12</smiles>

Quercetin<smiles>O=c1c(O)c(-c2cc(O)c(O)c(O)c2)oc2cc(O)cc(O)c12</smiles>

Myricetin

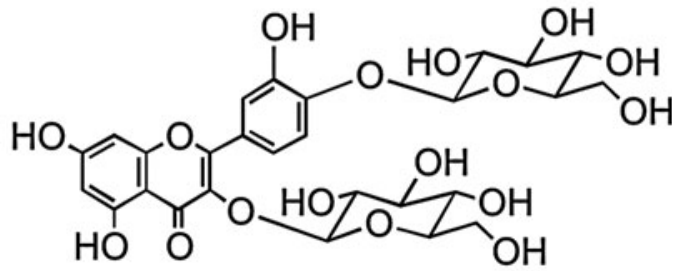

\section{Quercetin-3,4'-O-diglucoside}

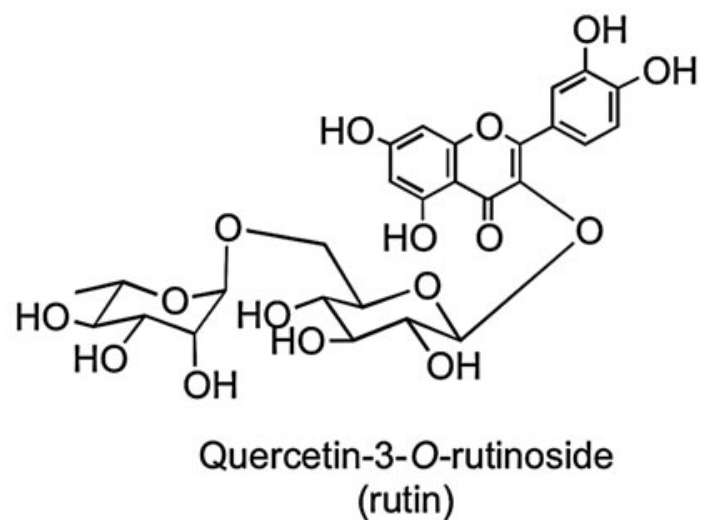

FIG. 2. Structures of the flavonol aglycones kaempferol, quercetin, isorhamnetin, and myricetin and three common quercetin-O-glycosides.

complex subclass of flavonoids, ranging from the simple monomers to the oligomeric and polymeric proanthocyanidins, which are also known as condensed tannins. The two chiral centers at C2 and C3 of the monomeric flavan-3-ol (Fig. 1) produce four isomers for each level of B-ring hydroxylation, two of which, (+)-catechin and (-)-epicatechin, are widespread in nature, while others such as $(-)$-epiafzelechin have a more limited distribution (Fig. 8) (12, 93). Pairs of enantiomers can be resolved by chiral chromatography, but not with the more commonly used reversed-phase high-performance liquid chromatography (HPLC), and as a consequence, they are easily overlooked.
Oligomeric and polymeric proanthocyanidins have an additional chiral center at $\mathrm{C} 4$ in the upper and lower units. Type B proanthocyanidins are formed from (+)-catechin and (-)epicatechin by oxidative coupling between the C-4 of the upper monomer and the C-6 or C-8 of the adjacent lower or extension unit to create oligomers or polymers. Type A proanthocyanidins have an additional ether bond between $\mathrm{C}$ 2 in the B-ring of one monomer and C-7 in the A-ring of the other monomer (Fig. 9). Proanthocyanidins can occur as polymers of up to 50 units. Proanthocyanidins that consist exclusively of (epi)catechin units are called procyanidins, and are the most abundant type of proanthocyanidins in plants. 
<smiles>O=c1cc(-c2ccc(O)cc2)oc2cc(O)cc(O)c12</smiles>

Apigenin<smiles>O=c1cc(-c2ccc(O)c(O)c2)oc2cc(O)cc(O)c12</smiles>

Luteolin<smiles>O=c1cc(-c2ccccc2)oc2cc(O)c(O)c(O)c12</smiles>

Baicalein<smiles>COc1c(O)cc(O)c2c(=O)cc(-c3ccccc3)oc12</smiles>

Wogonin<smiles>O=c1cc(-c2ccc(O)c(O)c2)oc2c(C3OC(CO)C(O)C(O)C(O)C3O)c(O)cc(O)c12</smiles>

Luteolin-8-C-glucoside (orientin)<smiles></smiles>

Apigenin-8-C-glucoside (vitexin)<smiles>COc1ccc(-c2cc(=O)c3c(OC)c(OC)c(OC)c(OC)c3o2)cc1OC</smiles>

Nobiletin

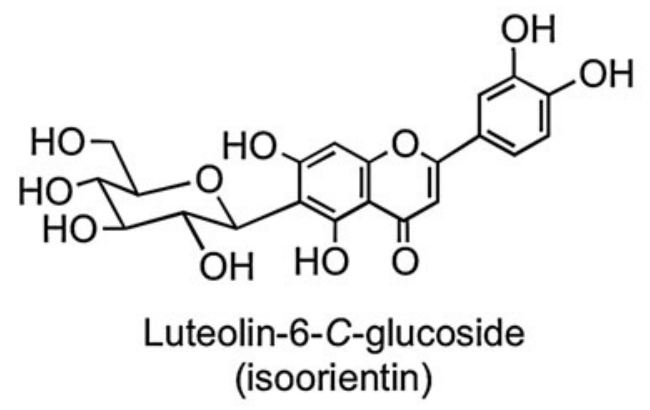<smiles>O=c1cc(-c2ccc(O)cc2)oc2cc(O)c(C(O)OCC(O)C(O)CO)c(O)c12</smiles>
(isovitexin)<smiles>COc1ccc(-c2cc(=O)c3c(OC)c(OC)c(OC)c(OC)c3o2)cc1</smiles>

Tangeretin

FIG. 3. The flavone aglycones apigenin, luteolin, baicalein, and wogonin; the flavone $C$-glycosides orientin, isoorientin, vitexin, and isovitexin; and the polymethoxylated flavones nobiletin and tangeretin.

The less-common proanthocyanidins containing (epi)afzelechin or (epi)gallocatechin subunits are propelargonidins and prodelphinidins, respectively (80).

Green tea (Camellia sinensis) contains very high levels of flavan-3-ol monomers with the main components being (-)-epigallocatechin, (-)-epigallocatechin-3-O-gallate, and (-)-epicatechin-3-O-gallate (Fig. 9). The levels of these flavan- 3-ols decline during fermentation of the green leaves to produce black tea, principally as a result of the action of polyphenol oxidase, and there is a concomitant accumulation of theaflavins and thearubigins (110). Theaflavin, theaflavin-3O-gallate, theaflavin-3'-O-gallate, and theaflavin-3,3'-Odigallate are dimer-like structures (Fig. 9) that contribute to the quality of the black tea beverage. The brownish, water-soluble, 
<smiles>O=C(O)c1ccc(O)cc1C(=O)c1ccc(O)cc1</smiles>

Daidzein<smiles>O=C(O)C12OC(C(O)=C1O)C(O)C2c1ccc(O)cc1</smiles>

Genistein

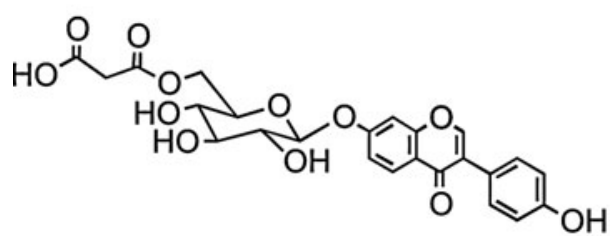

Daidzein-7-0-(6"-O-malonyl)glucoside

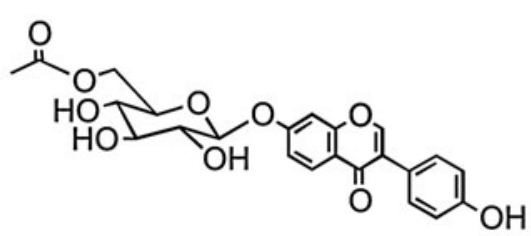

Daidzein-7-O-(6"-O-acetyl)glucoside

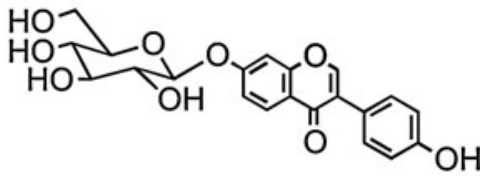

Daidzein-7-O-glucoside

FIG. 4. The isoflavone aglycones daidzein and genistein and $O$-glycosides of daidzein, which along with similar genistein conjugates, are found in soy products.

high-molecular-weight thearubigins are the major phenolic fraction in black tea. Recent pioneering studies have established that black teas contain on average 5000 thearubigin components in the mass range of 1000-2100 amu $(261,262)$. A typical cup of black tea contains $\sim 100 \mathrm{mg}$ of thearubigins $(164,499)$.
7. Dihydrochalcones. This minor group of flavonoids, which have an open C-ring structure, is of limited dietary significance, restricted to the presence of phloretin-2'-Oglucoside (phloridzin) and phloretin-2'-O-(2"-O-xylosyl) glucoside in apples (Malus domestica) and $2^{\prime}, 3,4,4^{\prime}, 6^{\prime}$ -<smiles>COc1cc(C[C@@H](CO)[C@H](CO)Cc2ccc(O)c(OC)c2)ccc1O</smiles>

Secoisolariciresinol<smiles>COc1cc(C[C@@H]2C(=O)OC[C@@H]2Cc2ccc(O)c(OC)c2)ccc1O</smiles>

Matairesinol<smiles>COc1cc([C@H]2OC[C@@H](c3ccc(O)c(OC)c3)[C@@H]2CO)ccc1O</smiles>

Pinoresinol<smiles>COc1cc(C[C@@H]2CO[C@H](c3ccc(O)c(OC)c3)[C@H]2CO)ccc1O</smiles>

Lariciresinol<smiles>COC[C@@H]1[C@@H](c2cc(OC)c(O)c(OC)c2)OC[C@H]1c1cc(OC)c(O)c(OC)c1</smiles>

Syringaresinol<smiles>c1cc([C@H]2CO[C@H](c3ccc4c(c3)OCO4)[C@H]2c2ccc3c(c2)OCO3)co1</smiles>

Sesamin<smiles>Oc1c([C@H]2[CH][C@@H](c3ccc4c(c3)OCO4)OC2)ccc2c1OCO2</smiles>

Sesaminol<smiles>COc1cc([C@@H]2CO[C@H]3CO[C@H](c4cc(OC)c(O)c(OC)c4)[C@@H]32)ccc1O</smiles>

Medioresinol<smiles>COc1ccc(C[C@H]2C(=O)OC[C@H]2Cc2ccc(O)c(OC)c2)cc1O</smiles>

7'-Hydroxymatairesinol

FIG. 5. Structures of plant lignans present in cereal grains and sesame seeds. 
<smiles>COc1ccc([C]2CC(=O)c3c(O)cc(O)cc3O2)cc1O</smiles>

Hesperetin<smiles>O=C1C[C@H](c2ccc(O)cc2)Oc2cc(O)cc(O)c21</smiles>

Naringenin<smiles></smiles>

Hesperetin-7-O-rutinoside (hesperidin)<smiles>COc1ccc([C@H]2CC(=O)c3c(O)cc(OC4OCC(CO)C(O)C(OC5OC(CO)C(O)C(O)C5O)C(O)C4O)cc3O2)cc1O</smiles>

Hesperetin-7-O-neohesperidoside (neohesperidin)

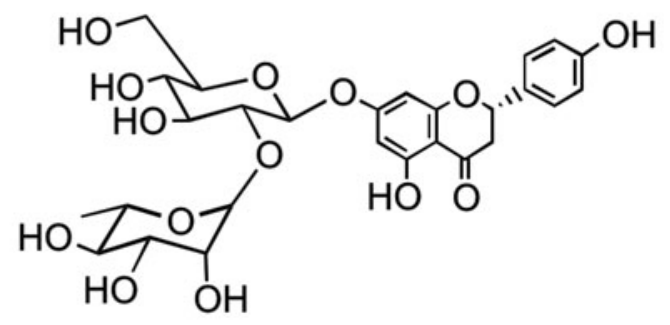

Naringenin-7-O-neohesperidoside (naringin)

FIG. 6. The S-enantiomers of the flavanone aglycones naringenin and hesperetin together with some of their naturally occurring glycosides.<smiles></smiles>

\begin{tabular}{lll}
\hline Anthocyanidin & $\mathbf{R}_{\mathbf{1}}$ & $\mathbf{R}_{\mathbf{2}}$ \\
\hline Pelargonidin & $\mathrm{H}$ & $\mathrm{H}$ \\
Cyanidin & $\mathrm{OH}$ & $\mathrm{H}$ \\
Delphinidin & $\mathrm{OH}$ & $\mathrm{OH}$ \\
Peonidin & $\mathrm{OCH}_{3}$ & $\mathrm{H}$ \\
Petunidin & $\mathrm{OCH}_{3}$ & $\mathrm{OH}$ \\
Malvidin & $\mathrm{OCH}_{3}$ & $\mathrm{OCH}_{3}$ \\
\hline
\end{tabular}

FIG. 7. Structures of the major anthocyanidins.
pentahydroxydihydrochalcone-3'-C-glucoside (aspalathin) and 2',4,4', $6^{\prime}$-tetrahydroxydihydrochalcone-3'-C-glucoside (nothofagin) (Fig. 10) in rooibos tea $(254,437)$.

\section{B. Nonflavonoids}

Among the nonflavonoids of dietary significance are the $\mathrm{C}_{6}-\mathrm{C}_{1}$ phenolic acids. Gallic acid is the commonest phenolic acid, and occurs widely as complex sugar esters in gallotannins, such as 2-O-digalloyl-tetra-O-galloyl-glucose (Fig. $11)$, which are minor dietary components. The related ellagic acid-based ellagitannins, such as sanguiin H-6 and punicalagin (Fig. 11), are found in a diversity of food, including raspberries (Rubus idaeus), strawberries (Fragaria ananassa), blackberries (Rubus spp.), and many other fruits, including pomegranate (Punica granatum) and persimmon (Diospyros kaki), as well as walnuts (Juglans regia), hazelnuts (Corylus avellana), and oak-aged wines where they are leached from the oak during maturation of the wines (267). The ellagitannin content of some food products can be high with a glass of pomegranate juice and a $100 \mathrm{~g}$ serving of raspberries providing $\sim 300 \mathrm{mg}$, and four walnuts $\sim 400 \mathrm{mg}$ (269)

The $\mathrm{C}_{6}-\mathrm{C}_{3}$ hydroxycinnamates occur mainly as conjugates, for example, with tartaric acid or quinic acid, and collectively 
FIG. 8. Structures of monomeric flavan-3-ol enantiomers.<smiles>Oc1cc(O)c2c(c1)O[C@H](c1ccc(O)c(O)c1)[C@H](O)C2</smiles>

(-)-Epicatechin<smiles>Oc1cc(O)c2c(c1)O[C@H](c1ccc(O)c(O)c1)[C@H](O)C2</smiles>

(+)-Epicatechin<smiles>Oc1cc(O)c2c(c1)O[C@H](c1ccc(O)c(O)c1)[C@H](O)C2</smiles>

$(+)$-Catechin<smiles>Oc1cc(O)c2c(c1)O[C@H](c1ccc(O)c(O)c1)[C@H](O)C2</smiles>

$(-)$-Catechin<smiles>Oc1ccc([C@@H]2Oc3cc(O)cc(O)c3C[C@H]2O)cc1</smiles>

(-)-Epiafzelechin<smiles>Oc1ccc([C@@H]2Oc3cc(O)cc(O)c3C[C@H]2O)cc1</smiles>

$(+)$-Epiafzelechin are referred to as chlorogenic acids (Fig. 12). Chlorogenic acids, principally 3-O-, 4-O-, and 5-O-caffeoylquinic acids, form $\sim 10 \%$ of green robusta coffee beans (Coffea canephora). Regular consumers of coffee may provide a daily intake in excess of $1 \mathrm{~g}$ of chlorogenic acids, and these for many people will be the major dietary phenolics. Accumulating in the flesh of grapes, caftaric acid is the main hydroxycinnamate in both red and white wines produced from Vitis vinifera and well as Concord grape juice, which is a product of grapes of Vitis lambrusca (435).

Stilbenes have a $\mathrm{C}_{6}-\mathrm{C}_{2}-\mathrm{C}_{6}$ structure and are phytoalexins produced by plants in response to disease, injury, and stress (268). Although only extremely minor dietary components, the main stilbene is resveratrol (3,5,4'-trihdroxystilbene), which occurs as cis and trans isomers as well as conjugated derivatives, including trans-resveratrol-3-O-glucoside (transpiceid) (Fig. 13). The woody root of the noxious weed Polygonum cuspidatum (Japanese knotweed or Mexican bamboo) contains unusually high levels of trans-resveratrol and its glucoside with concentrations of up to $377 \mathrm{mg} / 100 \mathrm{~g}$ dry weight (474). Red wines contain a diversity of stilbene derivatives, but invariably in very low concentrations compared to the levels of other (poly)phenolic components (92).

\section{Bioavailability of Flavonoids and Related Compounds}

The absorption, distribution, metabolism, and excretion of flavonoids and related phenolics after dietary intake have been topics of increasing research in recent years. After the acute ingestion, absorption of some, but by no means all, components into the circulatory system occurs in the small intestine. Typically, the absorption of flavonoid glycosides, as illustrated in Figure 14, is associated with cleavage and release of the aglycone as a result of the action of lactase phloridzin hydrolase (LPH) in the brush border of the small intestine epithelial cells. LPH exhibits broad substrate specificity for flavonoid-O- $\beta$-D-glucosides, and the released aglycone may then enter the epithelial cells by passive diffusion as a result of its increased lipophilicity and its proximity to the cellular membrane (104). An alternative hydrolytic step is mediated by a cytosolic $\beta$-glucosidase (CBG) within the epithelial cells.
For CBG-catalyzed hydrolysis to occur, the polar glucosides must be transported into the epithelial cells, possibly with the involvement of the active sodium-dependent glucose transporter 1 (SGLT1) (151). Thus, there are two possible routes by which the glycoside conjugates are hydrolyzed, and the resultant aglycones appear in the epithelial cells, namely LPH/ diffusion and transport/CBG (Fig. 14). However, an investigation in which SGLT1 was expressed in Xenopus laevis oocytes has shown that at least in this model system, SLGT1 does not transport flavonoids, and that glycosylated flavonoids and some aglycones have the capability to inhibit the glucose transporter (253). Using Caco-2 cells, Johnson et al. (228) found that glucose uptake into cells under sodiumdependent conditions was inhibited by flavonoid glycosides and nonglycosylated polyphenols, whereas aglycones and phenolic acids were without effect.

Before passage into the blood stream, the aglycones undergo some degree of phase II metabolism forming sulfate, glucuronide, and/or methylated metabolites through the respective action of sulfotransferases (SULTs), uridine-5'-diphosphate glucuronosyltransferases (UGT), and catechol-Omethyltransferases (COMTs). There is also efflux of some of the metabolites back into the lumen of the small intestine, and this is thought to involve members of the adenosine triphosphate-binding cassette (ABC) family of transporters, including multidrug resistance protein (MRP) and P-glycoprotein (Fig. 14). MRP-3 and the glucose transporter GLUT2 have also been implicated in the efflux of metabolites from the basolateral membrane of the enterocytes $(293,468)$. Once in the portal bloodstream, metabolites rapidly reach the liver, where they can be subjected to further phase II metabolism, and enterohepatic recirculation may result in some recycling back to the small intestine through bile excretion (118).

(Poly)phenol conjugates with sugar moieties that are resistant to the action of LPH/CBG (104) are not absorbed in the small intestine to any degree and pass to the colon. Analysis of ileal fluid collected from ileostomists after the ingestion of various foodstuffs has shown that even when dietary (poly)phenols are absorbed in the proximal GIT, substantial quantities nonetheless pass from the small to the large intestine $(220,235,236,294)$, where the colonic microbiota cleave 
<smiles>Oc1cc(O)c2c(c1)O[C@H](c1ccc(O)c(O)c1)[C@H](O)[C@H]2c1c(O)cc(O)c2c1O[C@H](c1ccc(O)c(O)c1)[C@H](O)C2</smiles>

Proanthocyanidin $\mathrm{B}_{2}$ dimer<smiles>Oc1cc(O)c2c(c1)O[C@H](c1cc(O)c(O)c(O)c1)[C@H](O)C2</smiles>

(-)-Epigallocatechin<smiles></smiles>

Theaflavin-3-O-gallate<smiles>Oc1cc(O)c2c(c1)O[C@H](c1ccc(O)c(O)c1)[C@H](O)[C@H]2c1c(O)cc2c(c1O)C[C@@H](O)[C@H](c1ccc(O)c(O)c1)O2</smiles>

Proanthocyanidin $\mathrm{B}_{5}$ dimer<smiles>O=C(O[C@H]1Cc2c(O)cc(O)cc2O[C@H]1c1cc(O)c(O)c(O)c1)c1cc(O)c(O)c(O)c1</smiles>

(-)-Epigallocatechin-3-O-gallate<smiles></smiles>

Theaflavin-3'-O-gallate

FIG. 9. Flavan-3-ol structures.

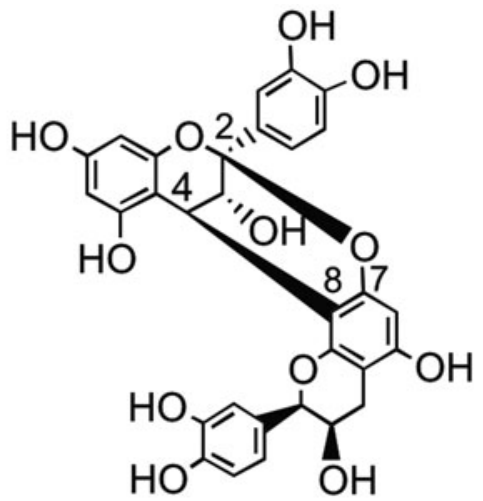

Proanthocyanidin $\mathrm{A}_{2}$ dimer<smiles>O=C(O[C@H]1Cc2c(O)cc(O)cc2O[C@H]1c1ccc(O)c(O)c1)c1cc(O)c(O)c(O)c1</smiles>

(-)-Epicatechin-3-O-gallate<smiles></smiles>

Theaflavin-3,3'-O-digallate 
<smiles>O=C(CCc1ccc(O)cc1)c1c(O)cc(O)cc1OC1OCC(O)C(CO)O1</smiles>

Phloretin-2'-O-glucoside<smiles>O=C(CCc1ccc(O)cc1)c1c(O)cc(O)cc1OC(CO)OC(O)CO</smiles>

FIG. 10. Naturally occurring dihydrochalcone glycosides.<smiles></smiles>

Aspalathin<smiles>O=C(CCc1ccc(O)cc1)c1c(O)cc(O)c(C(O)C(O)C(O)C(O)CO)c1O</smiles>

Nothofagin<smiles>O=C(O)c1cc(O)c(O)c(O)c1</smiles>

Gallic acid<smiles>O=c1oc2c(O)c(O)cc3c(=O)oc4c(O)c(O)cc1c4c23</smiles>

Ellagic acid<smiles>O=C(OOC(=O)C1OCCOC(OOC(=O)c2cc(O)c(O)c(OC(=O)c3cc(O)c(O)c(O)c3)c2)C1OOC(=O)c1cc(O)c(O)c(O)c1)c1cc(O)c(O)c(O)c1</smiles>

2-O-Digalloyl-tetra-O-galloyl-glucose

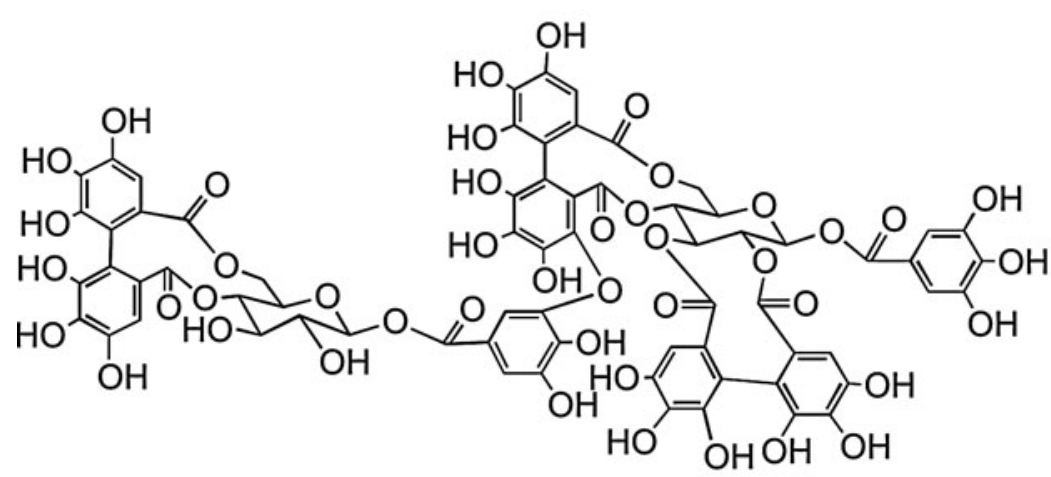

Sanguiin $\mathrm{H}-10$

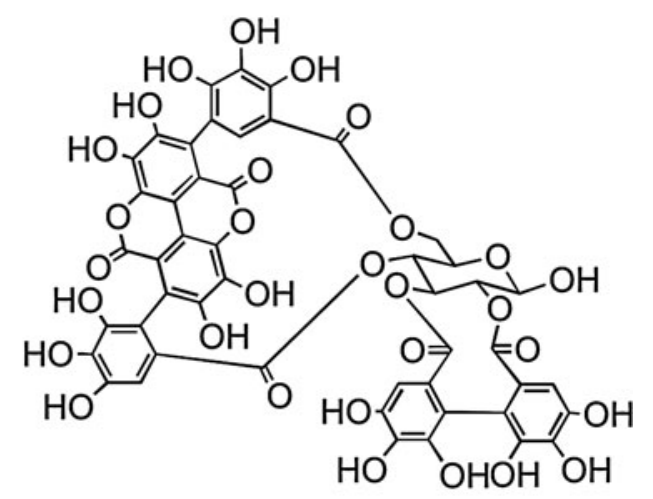

Punicalagin

FIG. 11. Gallic acid, ellagic acid, the gallotannin 2-O-digalloyl-tetra-O-galloyl-glucose, and the ellagitannins sanguiin $H-6$, and punicalagin. 
<smiles>O=C(O)C1(O)CC(O)C(O)C(O)CC(O)(C(=O)O)C1</smiles>

3-O-Caffeoylquinic acid<smiles>O=C(/C=C/c1ccc(O)c(O)c1)OC1C(O)CC(O)(C(=O)O)CC1O</smiles>

4-O-Caffeoylquinic acid<smiles>O=C(O)CC(O)C(O)CC(O)(CCCOC(=O)/C=C/c1ccc(O)c(O)c1)C(=O)O</smiles>

5-O-Caffeoylquinic acid<smiles>O=C(/C=C/c1ccc(O)c(O)c1)OC(C(=O)O)C(O)C(=O)O</smiles>

\section{Caftaric acid}

FIG. 12. The conjugated hydroxycinnamates 3-O-, 4-O-, and 5-O-caffeoylquinic acids and caftaric acid.

conjugating moieties, and the resultant aglycones undergo ring fission leading to the production of smaller molecules, including phenolic acids and hydroxycinnamates. These can be absorbed and may be subjected to metabolism in the liver before being excreted in urine in amounts that, in most instances, are well in excess of the metabolites that enter the circulatory system via the small intestine $(220,387,438,439)$.

As a result of more recent studies on the bioavailability of dietary (poly)phenolic compounds [see $(91,433)]$, there is a growing realization that (poly)phenolic glucuronide, methyl, and sulfate conjugates are treated by the body as xenobiotics, and instead of accumulating in the circulatory system, they are rapidly turned over and removed by excretion via the kidneys. As a consequence, although plasma pharmacoki-<smiles>Oc1ccc(C=Cc2cc(O)cc(O)c2)cc1</smiles>

trans-Resveratrol<smiles>Oc1ccc(/C=C\c2cc(O)cc(O)c2)cc1</smiles>

cis-Resveratrol<smiles>OCC1COC(Oc2cc(O)cc(C=Cc3ccc(O)cc3)c2)C(O)C(O)C1</smiles>

\section{trans-Resveratrol-3-O-glucoside}

FIG. 13. Common stilbene structures. netics of these metabolites provides useful information, estimates such as of area-under-the curve values do not necessarily yield accurate quantitative data on absorption. In the circumstances, urinary excretion provides a more realistic assessment. ${ }^{\dagger}$ However, as this does not include the possibility of sequestration in body tissues, this too is theoretically an underestimate of absorption, but to what degree remains undetermined. However, the fact that with few exceptions, tissue sequestration on any scale has yet to be convincingly demonstrated suggests that it can only be at low levels, if at all (see Section IV).

\section{A. Flavonols and flavanones}

A human feeding study with lightly fried onions containing a total of $275 \mu \mathrm{mol}$ of flavonol glucosides, principally in the form of quercetin-4'-O-glucoside and quercetin-3,4'-Odiglucoside (Fig. 2), resulted in the appearance of quercetin-3'$\mathrm{O}$-sulfate, quercetin-3-O-glucuronide, isorhamnetin-3-O-glucuronide (Fig. 15), and two partially identified metabolites, a quercetin-O-diglucuronide and a quercetin-O-glucuronide- $O$ sulfate, in the circulatory system within $30 \mathrm{~min}$ of ingestion (321). This is indicative of cleavage of the conjugating glucose moieties in the proximal GIT and metabolism of the released aglycone by SULTs, UGTs, and COMTs in the enterocyte before entry of the metabolites into circulatory system. Sub$\mu M$ peak plasma concentrations $\left(C_{\max }\right)$ were attained in $<1 \mathrm{~h}$ $\left(\mathrm{T}_{\max }\right)$, in the case of the majority of the metabolites, most of which had a relative short elimination half-life $\left(\mathrm{T}_{1 / 2}\right)$ as they

\footnotetext{
†Tis is because, as will be discussed later in the text, at a similar level of intake, the peak plasma concentration for quercetin metabolites can be $\sim 500 \mathrm{mM}$ and that of (-)-epicatechin metabolites $\sim 200 \mathrm{nM}$. In contrast, urinary excretion of the flavonol metabolites corresponds to $\sim 4 \%$ of intake, while that of the flavan-3-ol is $\sim 30 \%$ of the ingested dose.
} 
FIG. 14. Proposed mechanisms for the absorption and metabolism of (poly)phenolic compounds in the small intestine. $\mathrm{CBG}$, cytosolic $\beta$ glucosidase; COMT, catecholO-methyl transferase; GLUT2, glucose transporter; LPH, lactase phloridzin hydrolase; MRP1-2-3, multidrug-resistant proteins; PP, (poly)phenol aglycone; PP-gly, (poly)phenol glycoside, PP-met, polyphenol sulfate/glucuronide/methyl metabolites; SGLT1, sodiumdependent glucose transporter; SULT, sulfotransferase; UGT, uridine-5'-diphosphate glucuronosyltransferase. (To see this illustration in color, the reader is referred to the web version of this article at www.liebertpub.com/ars.)

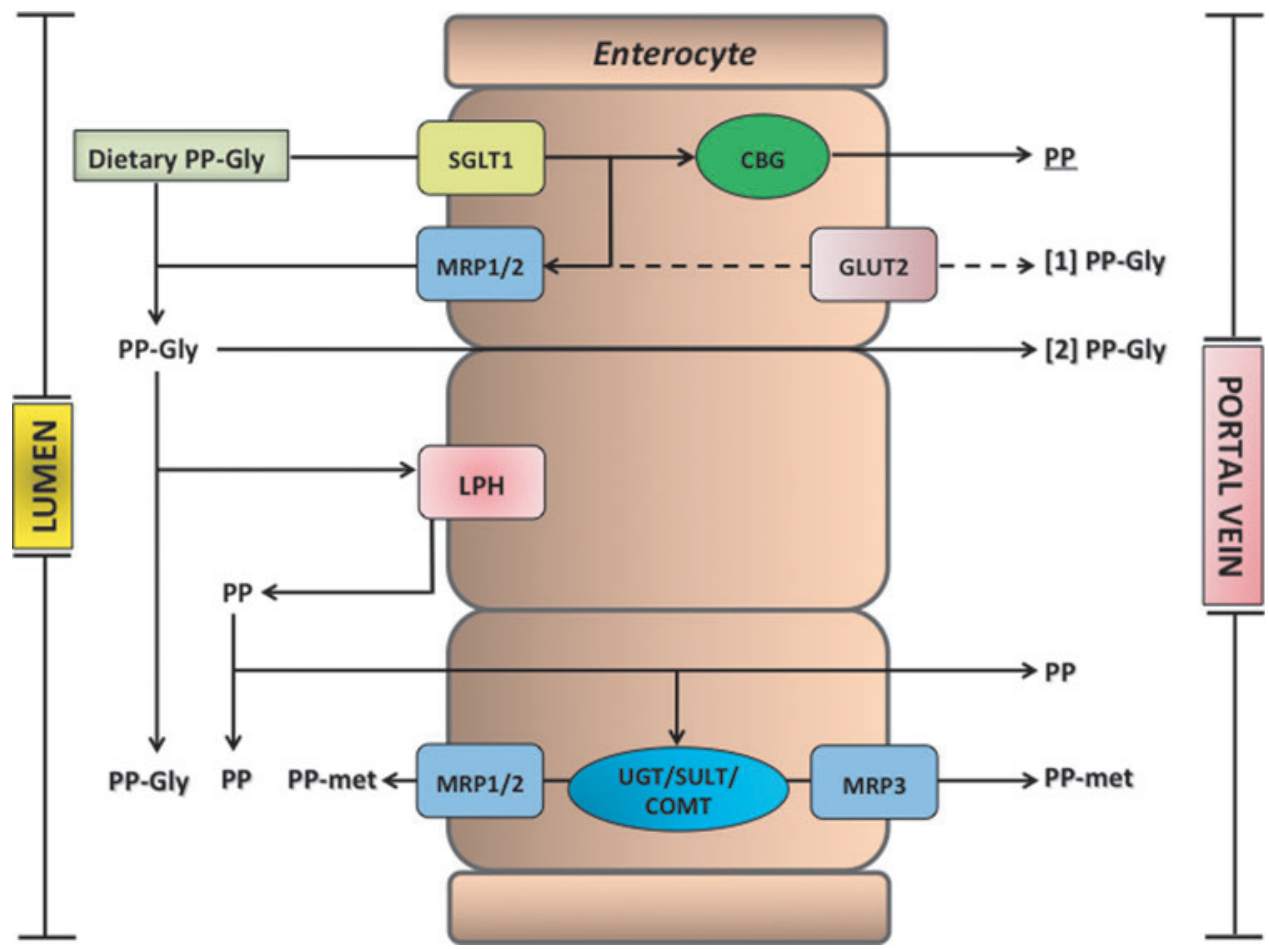

were rapidly removed from the bloodstream (Table 1). A more complex array of metabolites was detected in urine, indicating substantial phase II metabolism of the quercetin derivatives before excretion. Most of the metabolites were excreted within the initial $12 \mathrm{~h}$ after ingestion of the onions, and over a $0-24$-h collection period, a total of $12.9 \mu \mathrm{mol}$ of metabolites was excreted, which corresponds to $4.7 \%$ of intake.

After drinking tomato juice containing $176 \mu \mathrm{mol}$ of quercetin-3-O-rutinoside, quercetin-3-O-glucuronide and iso-
rhamnetin-3-O-glucuronide were detected in plasma (220). Their $C_{\max }$ values were $\sim 25$-fold lower than in the onion study, and the $T_{\max }$ times were extended to $\sim 5 \mathrm{~h}$ (Table 2 ), which is characteristic of absorption in the large rather than small intestine. Absorption in the large intestine was confirmed when the tomato juice was fed to ileostomists, as neither plasma nor urinary metabolites of quercetin were detected, and ileal fluid collected post-tomato juice consumption contained $86 \%$ of the ingested quercetin-3-O-
FIG. 15. Structures of the quercetin metabolites quercetin- $3^{\prime}-O$-sulfate, quercetin-3-O-glucuronide, and isorhamnetin-3-O-glucuronide $(105,321)$.

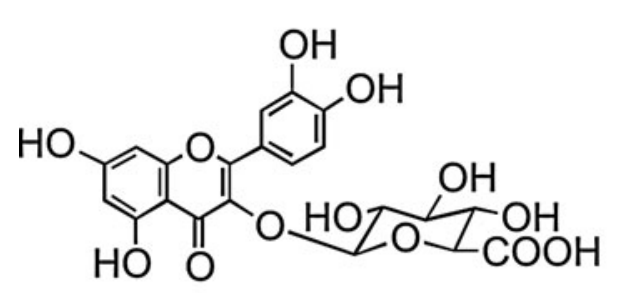

Quercetin-3-O-glucuronide<smiles>COc1cc(-c2oc3cc(O)cc(O)c3c(=O)c2OC(O)C2OC3OC2C(O)C(O)C(O)C3C(=O)O)ccc1O</smiles>

Isorhamnetin-3-O-glucuronide 
Table 1. Pharmacokinetic Analysis of Quercetin Metabolites in the Plasma of Volunteers After the Consumption of 270 g of Fried Onions Containing $275 \mu \mathrm{MOL}$ of Flavonol Glucosides

\begin{tabular}{lrrr}
\hline Metabolites & $C_{\max }(n \mathrm{M})$ & $T_{\max }(h)$ & $T_{1 / 2}(h)$ \\
\hline Quercetin-3'-O-sulfate & $665 \pm 82$ & $0.75 \pm 0.12$ & 1.71 \\
Quercetin-3-O-glucuronide & $351 \pm 27$ & $0.60 \pm 0.10$ & 2.33 \\
Isorhamnetin-3-O-glucuronide & $112 \pm 18$ & $0.60 \pm 0.10$ & 5.34 \\
Quercetin-O-diglucuronide & $62 \pm 12$ & $0.80 \pm 0.12$ & 1.76 \\
Quercetin-O-glucuronide- & $123 \pm 26$ & $2.5 \pm 0.22$ & 4.54 \\
$\quad$ & & & \\
$\quad$ O-sulfate & & &
\end{tabular}

Data presented as mean values \pm standard error $(n=6)$.

Adapted from Mullen et al. (321).

rutinoside. This is in keeping with the report of Day et al. (104) that the flavonol disaccharide is not cleaved by LPH. In healthy subjects, quercetin-3-O-rutinoside will pass from the small to large intestine, where cleavage of the sugar moiety occurs as a result of the action of the colonic microbiota, and the released aglycone undergoes low-level methylation and glucuronidation before absorption into the portal vein. Most of the quercetin, however, is subject to ring fission, resulting in the production of hydroxyphenylacetic acid catabolites (Fig. 16) in amounts equivalent to $\sim 22 \%$ of intake of the rutinoside. These catabolites were not excreted by ileostomists after ingestion of tomato juice containing quercetin-3-Orutinoside (220).

It is of interest to note that quercetin released in the small intestine by cleavage of quercetin glucosides is converted to glucuronide, sulfate, and methylated metabolites (321), whereas quercetin produced in the colon from quercetin-3-Orutinoside is metabolized to methyl and glucuronide derivatives, but not sulfate metabolites (220). This suggests that sulfation of quercetin is a feature of SULTs in the wall of the small intestine rather than the colon or the liver.

Flavanone bioavailability studies with healthy human subjects have been carried out using a $250-\mathrm{ml}$ supplement of orange (Citrus sinensis) juice containing $168 \mu \mathrm{mol}$ of hesperetin-7-Orutinoside and $12 \mu \mathrm{mol}$ of naringenin-7-O-rutinoside (319). The hesperetin-7-O-rutinoside dose was therefore very similar to that of the quercetin-3-O-rutinoside in the tomato juice study (220). Plasma contained hesperetin-7-O-glucuronide (Fig. 17) and a second unassigned hesperetin-O-glucuronide, and the combined $\mathrm{C}_{\max }$ for the metabolites was $922 \mathrm{nM}$ at a $\mathrm{T}_{\max }$ of $4.4 \mathrm{~h}$. The two hesperetin metabolites were also excreted in urine along with a third hesperetin-O-glucuronide, two hesperetin- $O$ glucuronide-O-sulfates, and a hesperetin-O-diglucuronide. These marked differences in the plasma and urinary hesperetin metabolite profiles demonstrate that substantial postabsorption phase II metabolism is occurring. The quantities of metabolites excreted $0-24 \mathrm{~h}$ after ingestion corresponded to $6.5 \%$ of hesperetin-7-O-rutinoside intake. Although no naringenin metabolites were detected in plasma, urine contained naringenin-7-O-glu-

\footnotetext{
In a subsequent orange juice feeding study, urinary hesperetin metabolites that were identified were hesperetin-7-O-glucuronide, hesperetin-5-O-glucuronide, hesperetin-3'-O-glucuronide, hesperetin5,7-O-diglucuronide, hesperetin-3,7-O-diglucuronide, and hesperetin$3^{\prime}$-O-sulfate along with a hesperetin-O-glucuronide-O-sulfate (Fig. 17) (46).
}

Table 2. Pharmacokinetic Analysis of Quercetin Metabolites in the Plasma of Volunteers After the Consumption of 250 ML of Tomato Juice Containing $176 \mu$ mol of Quercetin-3-O-Rutinoside

\begin{tabular}{lcc}
\hline Metabolites & $C_{\max }(n \mathrm{M})$ & $T_{\max }(h)$ \\
\hline Quercetin-3-O-glucuronide & $12 \pm 2$ & $4.7 \pm 0.3$ \\
Isorhamnetin-3-O-glucuronide & $4.3 \pm 1.5$ & $5.4 \pm 0.2$
\end{tabular}

Data presented as mean values \pm standard error $(n=6)$.

Adapted from Jaganath et al. (220).

curonide, narigenin-4'-O-glucuronide (Fig. 17), and a naringenin-O-diglucuronide in amounts equivalent to $17.3 \%$ of the ingested naringenin-7-O-rutinoside. The differing levels of excretion of hesperetin and naringenin metabolites, relative to the amounts ingested, is a trend that has been observed in some, but not all, flavanone-feeding studies (291). While it could be a dose effect reflecting the higher intake of the hesperetin conjugate, it is more likely to be due to naringenin-7-O-rutinoside being more bioavailable than hesperetin-7-O-rutinoside, indicating that the $3^{\prime}$ and $4^{\prime}$ substituents impact on absorption.

Although both are absorbed in the large intestine, the $922 \mathrm{nM} \mathrm{C}_{\max }$ of the hesperetin-O-glucuronides is more than 50 -fold higher than that of the quercetin-3-O-rutinoside metabolites despite the amounts ingested being similar. This, coupled with the higher level of excretion of the orange juice metabolites, indicates that hesperetin-7-O-rutinoside metabolites are absorbed from the large intestine much more effectively than those of quercetin-3-O-rutinoside. This may be a consequence of the hesperetin-7-O-rutinoside being converted to glucuronides in the large intestine more efficiently than quercetin-3-O-rutinoside, perhaps because it is less prone to degradation by colonic bacteria. Among the urinary hesperetin metabolites were two $\mathrm{O}$-glucuronide- $\mathrm{O}$-sulfates. This contrasts with the absence of both sulfated naringenin metabolites and sulfated quercetin metabolites derived from large intestine absorption of quercetin-3-O-rutinoside in the tomato juice feed (Section III.A). Thus, there appear to be clear differences in the substrate specificity of flavonoid SULTs in the large intestine and/or the liver.

Analysis of phenolic acids and aromatic compounds excreted in urine after the ingestion of orange juice indicates that the hesperetin, released through colonic bacterium-mediated deglycosylation, as well as being glucuronidated, undergoes ring fission and is catabolized, producing 3(3'-hydroxyphenyl) hydracrylic acid, 3(3'-methoxy-4'-hydroxyphenyl)hydracrylic acid, 3(3'-methoxy-4'-hydroxyphenyl)propionic acid (dihydroferulic acid) 3'-hydroxyphenylacetic acid, and 3'-hydroxyhippuric acid (Fig. 18) (386). The overall level of the five phenolic acids excreted $0-24 \mathrm{~h}$ after drinking water was $6.7 \mu \mathrm{mol}$, and this rose to $62 \mu \mathrm{mol}$, equivalent to $37 \%$ of the ingested flavanones, after orange juice consumption.

\section{B. Anthocyanins}

Anthocyanins can be major dietary components with intakes in excess of $1 \mathrm{~g}$ being feasible for those who routinely eat berries and drink red wine. Unlike other flavonoids that are absorbed and excreted, they do not appear to undergo extensive metabolism to glucuronide and sulfate derivatives. In feeding studies with humans, typical recoveries of 


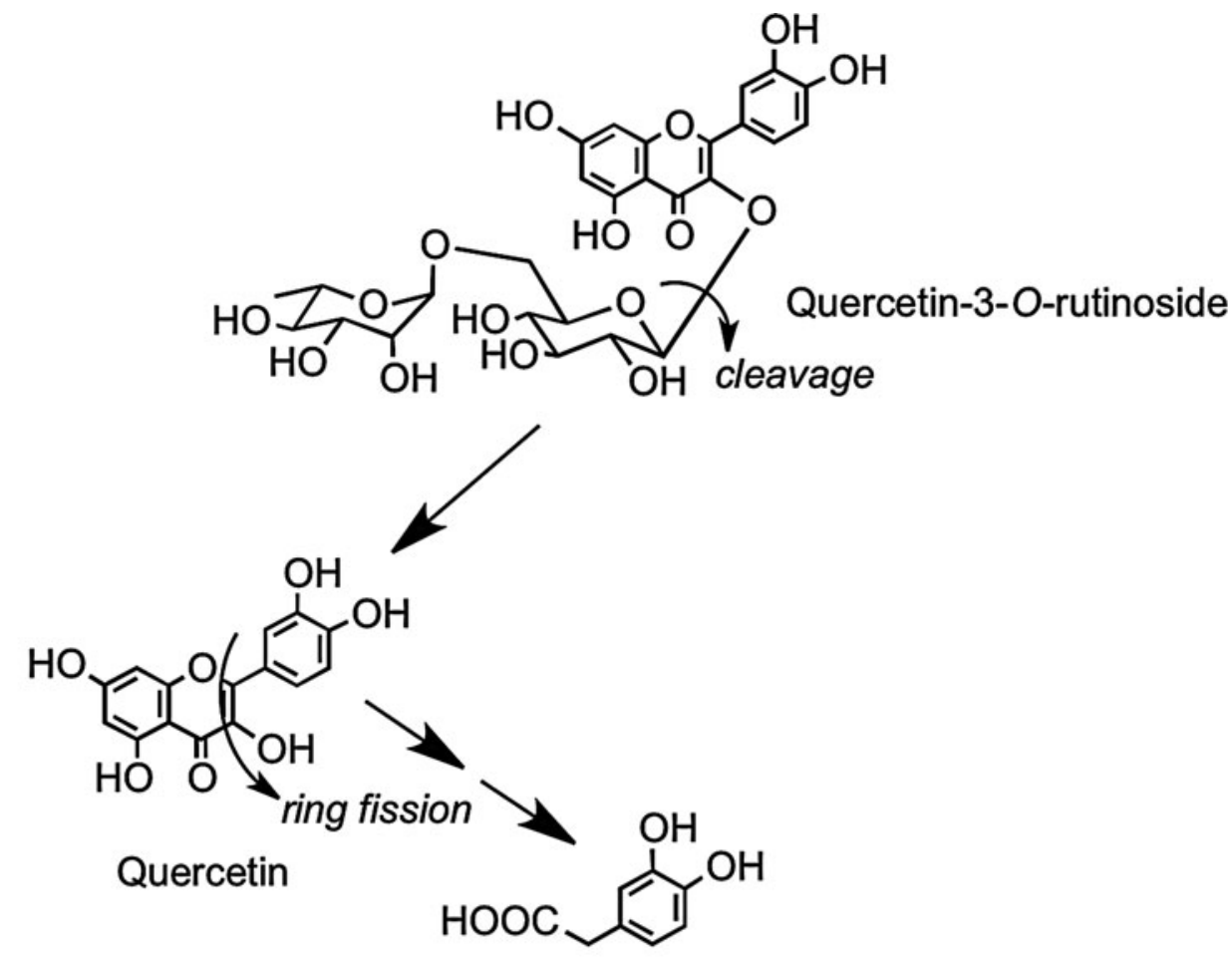

3',4'-Dihydroxyphenylacetic acid

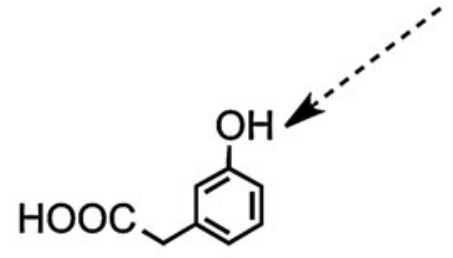

3'-Hydroxyphenylacetic acid

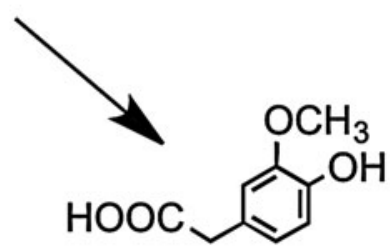

3'-Methoxy-4'-hydroxyphenylacetic acid

FIG. 16. Proposed pathway for colonic bacterium-mediated catabolism of quercetin-3-O-rutinoside in the human large intestine resulting in the production of $3^{\prime}, 4^{\prime}$-dihydroxyphenylacetic acid and smaller quantities of $3^{\prime}$-hydroxyphenylacetic acid with subsequent hepatic conversion of $3^{\prime}, 4^{\prime}$-dihydroxyphenylacetic acid to $3^{\prime}$-methyoxy-4'-hydroxyphenylacetic acid before urinary excretion. Adapted from Jaganath et al. (220).

anthocyanins in urine are $<0.1 \%$ of intake (109). For instance, $\mathrm{Wu}$ et al. (504) report a urinary recovery of blueberry (Vaccinium corymbosum) anthocyanins fed to elderly women of $0.004 \%$. To some degree, these low recoveries could be a consequence of anthocyanins undergoing structural rearrangements in response to $\mathrm{pH}$. The red flavylium cation predominates at $\mathrm{pH} 1-3$, but as the $\mathrm{pH}$ increases to 4 and above, the colorless carbinol pseudobase becomes the major component along with smaller amounts of a colorless chalcone pseudobase and a blue quinoidal base (78). Such changes are likely to occur in vivo as anthocyanins pass from a low $\mathrm{pH}$ in the stomach to the more-basic conditions of the small intestine.

The available evidence implies that the limited absorption and excretion of anthocyanins that does take place are influenced by the nature of both the sugar moiety and the anthocyanidin structure $(299,361,505)$. There are much complex data on the limited bioavailability of anthocyanins, which is, in part, due to the use of supplements that contain several structurally diverse anthocyanins. For instance, raspberries (Rubies idaeus) contain 10 or more anthocyanins as cyanidinand pelargonidin-3-O-glycosides ranging from mono- to trisaccharides, while more than 14 anthocyanins comprising mainly 3-O-glucoside, galactosides, and arabinosides of cyanidin, delphinidin, petunidin, and malvidin occur in blueberries (41). This makes the trace levels of complex anthocyanins in plasma and urine exceedingly difficult, if not impossible, to interpret in terms of absorption, metabolism, excretion, and potential phase I and phase II metabolism. Especially so when $3^{\prime}-O$-methylation can convert cyanidin to peonidin, and delphinidin to petunidin and 5'-O-methylation results in the metabolism of petunidin to malvidin.

Comparatively, simple anthocyanin profiles are found in strawberries and blackberries (Rubus fruticosa), as they contain one predominant anthocyanin, pelargonidin-3-Oglucoside, in the former and cyanidin-3-O-glucoside in the latter. After feeding strawberries to volunteers, the main component in plasma was a pelargonidin-O-glucuronide, 


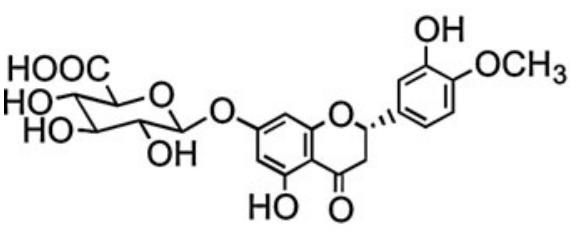

Hesperetin-7-O-glucuronide

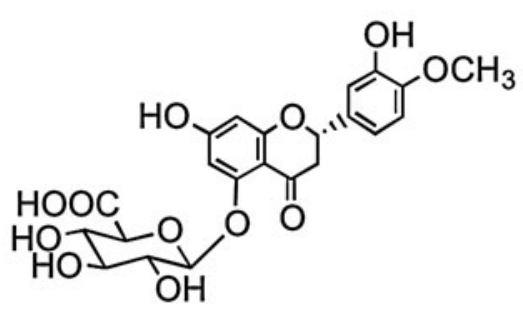

Hesperetin-5-O-glucuronide<smiles>COc1ccc([C@@H]2CC(=O)c3c(O)cc(O)cc3O2)cc1OC1OC(O)C(O)C(O)C(O)C1C(=O)O</smiles>

Hesperetin-3'-O-glucuronide

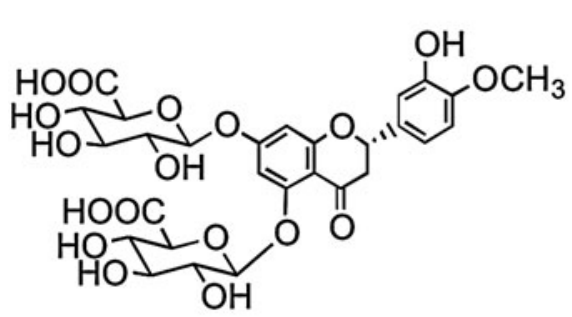

Hesperetin-5,7-O-diglucuronide

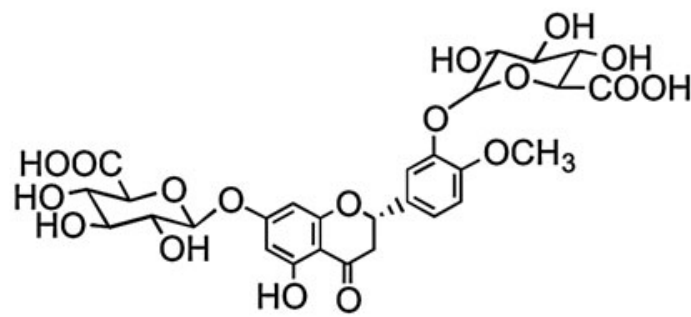

Hesperetin-3',7-O-diglucuronide<smiles>COc1ccc([C@@H]2CC(=O)c3c(O)cc(O)cc3O2)cc1OC</smiles>

Hesperetin-3'-O-sulfate

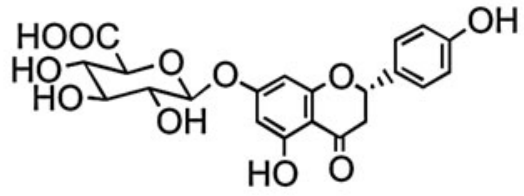

Naringenin-7-O-glucuronide

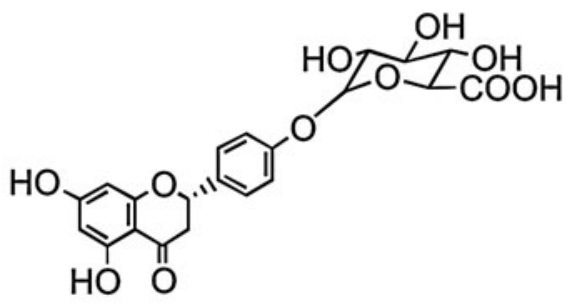

Naringenin-4'-O-glucuronide

FIG. 17. Narigenin and hesperetin metabolites $(46,49,319)$.

with plasma $\mathrm{C}_{\max }$ of $274 \mathrm{nM}$ and a $\mathrm{T}_{\max }$ of $1.1 \mathrm{~h}$, indicating absorption from the small intestine, whereas urinary excretion of the glucuronide corresponded to $0.75 \%$ of pelargonidin-3-O-glucoside intake (322). In other strawberry feeding studies, urinary excretion of $\sim 1.8 \%$ of the ingested pelargonidin-3-O-glucoside was obtained $(62,139)$. After feeding blackberries, 12 anthocyanins were detected in urine, including unmetabolized cyanidin-3-O-glucoside, a cyanidinO-glucuronide, and a peonidin-O-glucuronide, in quantities equivalent to $\sim 0.18 \%$ of intake. It, therefore, appears that the 3'-hydroxyanthocyanin, pelargonidin-3-O-glucoside, is metabolized to fewer products and may be absorbed more readily that its $3^{\prime}, 4^{\prime}$-dihydroxy analog, cyanidin-3-O-glucoside. This is in keeping with the evidence obtained with other flavonoids that the presence of a substituent group at the $3^{\prime}$ and $4^{\prime}$-positions can influence absorption. For instance, kaempferol with a $4^{\prime}$-hydroxyl group is more bioavailable than quercetin, which has a $3^{\prime}, 4^{\prime}$-dihydroxy structure (Fig. 2) $(38,125)$. Also, as noted above, naringenin with a $4^{\prime}$-hydroxyl group is absorbed more readily than hesperetin, which has $3^{\prime}$ hydroxyl and 4'-methoxy groups (Fig. 6).

Consumption of raspberries by ileostomists has shown that $40 \%$ of anthocyanin intake remained in the ileal fluid, although the recovery of individual compounds varied greatly ranging from $5.9 \%$ for cyanidin-3-O-glucoside to $93 \%$ for cy-
anidin-3-O-(2"-O-xylosyl)rutinoside (158). Analysis of urine collected from healthy volunteers after raspberry consumption and incubation of raspberry anthocyanins in fecal slurries under anaerobic conditions indicate that in vivo, the cyanidin-based anthocyanins are degraded by the colonic microbiota to a diversity of phenolic and aromatic catabolites by the pathways illustrated in Figure 19 (159). Vitaglione et al. have reported that protocatechuic acid (3,4-dihydroxybenzoic acid) is the principal catabolite of cyanidin-3-O-glucoside in humans (478).

Further complicating matters, anthocyanins also breakdown to phenolic acid and aldehyde constituents when subjected to simulated physiological conditions and during sample processing before analysis. In this regard, experiments with pelargonidin, cyanidin, and delphinidin showed that increased B-ring hydroxylation is associated with decreased in vitro stability (500).

\section{Flavones}

Compared to other flavonoids, there have been relatively few human feeding studies involving naturally occurring flavones. After feeding an infusion of the Chinese medicinal herb medicine Scutellariae Radix, which contains the flavones baicalein and wogonin (Fig. 3) and their 7-O-glucuronides, 36-h urinary excretion of baicalein metabolites was 
<smiles>COc1cc(C(O)CC(=O)O)ccc1O</smiles><smiles>O=C(O)C[C@H](O)c1cccc(O)c1</smiles>

\section{3-(3'-Methoxy-4'-hydroxyphenyl)hydracrylic acid}

\section{3-(3'-hydroxyphenyl)hydracrylic acid}<smiles>COc1cc(CCC(=O)O)ccc1O</smiles>

3-(3'-Methoxy-4'-hydroxyphenyl)propionic acid (dihydroferulic acid)<smiles>O=C(O)Cc1cccc(O)c1</smiles>

3'-Hydroxyphenylacetic acid<smiles>O=C(O)NC(=O)c1cccc(O)c1</smiles>

3'-Hydroxyhippuric acid

FIG. 18. Colonic phenolic acid catabolites of hesperetin.

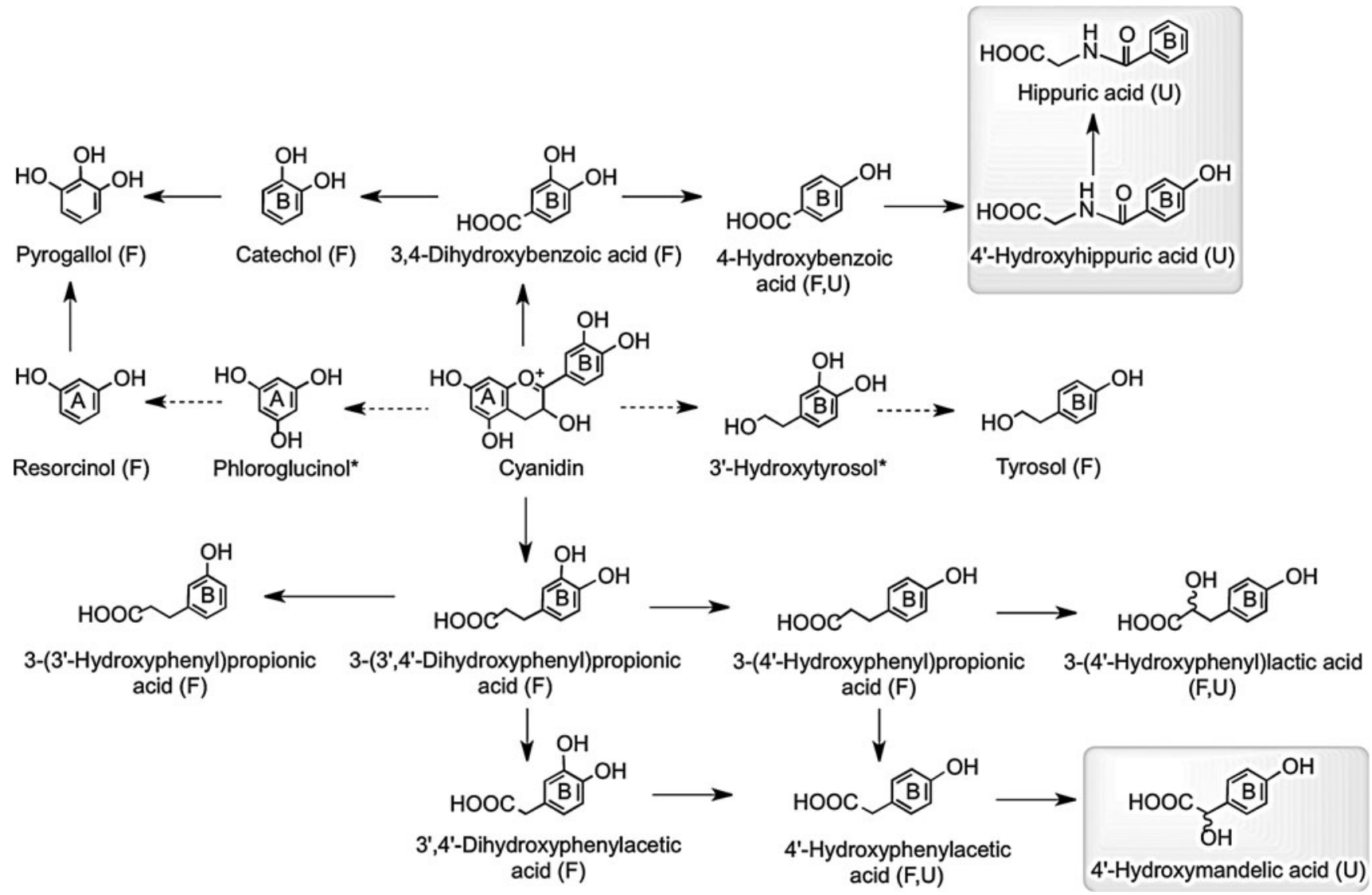

FIG. 19. Proposed pathways for the conversion of cyanidin-based red raspberry anthocyanins to phenolic acids and aromatic compounds. After consumption of $300 \mathrm{~g}$ of raspberries $\sim 40 \%$ of the ingested anthocyanins, principally cyanidin-Oglycosides, pass into the large intestine. When raspberry anthocyanins are incubated with fecal suspensions under anaerobic conditions, the cyanidin aglycone is released and catabolized by the colonic microflora undergoing C-ring fission, releasing phenolic acids, originating from both the A- and B-rings, which are metabolized via the pathways illustrated. Analysis of urine collected after raspberry consumption indicates that some of the colonic catabolites enter the circulatory and undergo further metabolism before being excreted in urine. These catabolites are thus detected in urine, but not fecal suspensions. $\mathrm{F}$, catabolites detected in fecal suspensions; $\mathrm{U}$, catabolites detected in urine (also highlighted in gray); ${ }^{*}$ potential intermediates that did not accumulate in detectable quantities. Adapted from Gonzalez-Barrio et al. (159). 
$4.0 \%$ of intake compared to $7.1 \%$ for wogonin metabolites (265). This implies that the presence of a methoxy group at C-8 reduces flavone absorption by $\sim 50 \%$. After ingestion of unfermented and fermented rooibos teas, which contain low levels of the flavone C-linked conjugates, vitexin, isovitexin, orientin, and iso-orientin (Fig. 3), no flavones or flavone metabolites were detected in either plasma or urine (437). This is probably a reflection of these compounds not being absorbed in either the small or the large intestine, and that the colonic microbiota were unable to cleave the C-linked sugar moiety.

\section{Isoflavones}

After consumption of soy milk containing mainly the 7-Oglucosides of daidzein and genistein (Fig. 4), Setchell et al. (410) detected urinary isoflavones as deglycosylated sulfates and glucuronides, implying the involvement of intestinal metabolism in their bioavailability. In a subsequent study, the same group fed several doses of $\left[4-{ }^{13} \mathrm{C}\right]$ daidzein and $\left[4-^{13} \mathrm{C}\right]$ genistein to premenopausal women (411). Metabolites of both $\left[{ }^{13} \mathrm{C}\right]$ isoflavones began appearing in plasma within $1 \mathrm{~h}$ of feeding, and sub- $\mu M \mathrm{C}_{\max }$ values were associated with $\mathrm{T}_{\max }$ times of $5.5 \mathrm{~h}$ for genistein and $7.4 \mathrm{~h}$ for daidzein metabolites, and $\mathrm{T}_{1 / 2}$ times of $\sim 7.7 \mathrm{~h}$. This suggests that the isoflavone metabolites are being absorbed into the circulatory system from both the small and large intestine. Urinary recoveries were $31 \%$ for $\left[{ }^{13} \mathrm{C}\right]$ daidzein and $9.0 \%$ for $\left[{ }^{13} \mathrm{C}\right]$ genistein, demonstrating the reduced bioavailability of the 5-hydroxylated isoflavone.

In a more recent investigation, Hosoda et al. (213) fed kinako, baked soybean flour, containing daidzein-7-Oglucoside $(13.2 \mu \mathrm{mol})$, daidzein $(23.9 \mu \mathrm{mol})$, genistein-7-Oglucoside $(21.2 \mu \mathrm{mol})$, and genistein $(41.0 \mu \mathrm{mol})$ to human volunteers. Plasma and urine collected over a 48-h postingestion period contained the aglycone daidzein, daidzein4'-O-glucuronide, daidzein-7-O-glucuronide, daidzein-4',7$O$-diglucuronide, daidzein-4'-O-sulfate, daidzein-7-O-sulfate, and daidzein-4'-O-sulfate-7-O-glucuronide (Fig. 20) together with a similarly comprehensive spectrum of genistein metabolites. Plasma $\mathrm{T}_{\max }$ times ranged from 1.6 to $17.1 \mathrm{~h}$, indicating that absorption seemingly takes place in both the proximal and distal GIT. Although no individual component attained a $\mu M \mathrm{C}_{\max }$, urinary excretion of genistein metabolites was $29.5 \%$ of intake and that of daidzein derivatives $72.1 \%$ (Table 3), confirming that isoflavones, especially daidzein, are highly bioavailable compared to most other (poly)phenols.

In vitro anaerobic incubations with human feces have shown two routes of metabolism for daidzein, depending on subjects and their gut microbiota. Some subjects produce equol via dihydrodaidzein and tetrahydrodaidzein, and others produce $O$-desmethylangolensin via 2'-dehydro-Odesmethylangolensin (Fig. 21). Thus, there are two subpopulations: those who have the microbiota capable of<smiles>O=c1c(-c2ccc(O)cc2)coc2cc(O)ccc12</smiles>

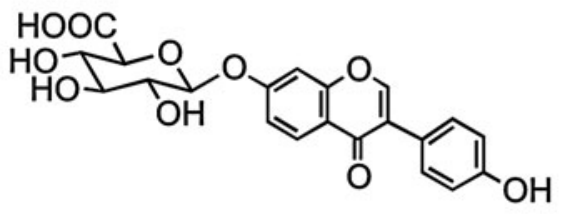

Daidzein-7-O-glucuronide<smiles>O=c1c(-c2ccc(O)cc2)coc2cc(OS(=O)(=O)[O-])ccc12</smiles>

Daidzein-7-O-sulfate<smiles>O=C(O)C1C2COC(OC(Oc3ccc(-c4coc5cc(O)ccc5c4=O)cc3)C2O)C1(O)O</smiles>

Daidzein-4'-O-glucuronide<smiles>COS(=O)(=O)Oc1ccc(-c2coc3cc(O)ccc3c2=O)cc1</smiles>

Daidzein-4'-O-sulfate

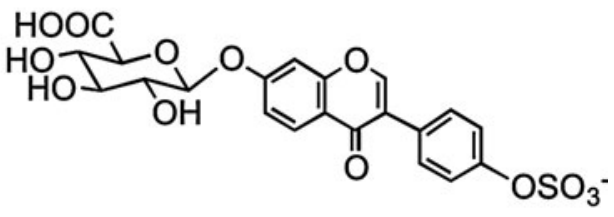

Daidzein-4',7-O-diglucuronide

Daidzein-4'-O-sulfate-7-O-glucuronide

FIG. 20. Daidzein metabolites detected in plasma and urine after acute supplementation with baked soy flour in milk. A similar array of genistein metabolites was also detected. Adapted from Hosoda, et al. (213). 
Table 3. Pharmacokinetic Analysis of Isoflavone Metabolites in the Plasma of Volunteers After the Consumption of 10 g of Baked Soybean Flour Containing $275 \mu$ mol of Daidzein, Genistein, AND Their 7-O-GLucosides

\begin{tabular}{lcrr}
\hline Isoflavones & $T_{\max }(h)$ & $C_{\max }(\mu \mathrm{M})$ & Excretion $(\mu \mathrm{mol})$ \\
\hline Daidzein & $2.3 \pm 0.5$ & $0.01 \pm 0.01$ & $0.39 \pm 0.01$ \\
Daidzein-4'-O-glucuronide & $4.5 \pm 0.8$ & $0.10 \pm 0.02$ & $4.65 \pm 0.55$ \\
Daidzein-7-O-glucuronide & $6.1 \pm 0.8$ & $0.09 \pm 0.01$ & $12.86 \pm 0.99$ \\
Daidzein-4',7-O-diglucuronide & $2.9 \pm 0.5$ & $0.05 \pm 0.00$ & $3.26 \pm 0.81$ \\
Daidzein-4'-O-sulfate & $4.1 \pm 1.0$ & $0.04 \pm 0.00$ & $0.57 \pm 0.1$ \\
Daidzein-7-O-sulfate & $1.6 \pm 0.4$ & $0.09 \pm 0.01$ & $0.70 \pm 0.13$ \\
Daidzein-4'-O-sulfate-7-O-glucuronide & $7.4 \pm 0.6$ & $0.34 \pm 0.30$ & $4.30 \pm 0.99$ \\
Total/mean & 4.7 & $0.64 \pm 0.06$ & $26.73 \pm 1.92(72.1 \%)$ \\
Genistein & $8.0 \pm 2.8$ & $0.01 \pm 0.01$ & $0.11 \pm 0.03$ \\
Genistein-4'-O-glucuronide & $3.9 \pm 0.8$ & $0.14 \pm 0.02$ & $4.95 \pm 1.02$ \\
Genistein-7-O-glucuronide-4'-O-glucuronide & $5.0 \pm 0.5$ & $0.11 \pm 0.01$ & $5.23 \pm 0.98$ \\
Genistein-4',7-O-diglucuronide-7-O-glucuronide & $6.8 \pm 0.9$ & $0.45 \pm 0.08$ & $3.66 \pm 0.74$ \\
Genistein-4'-O-sulfate-4',7-O-diglucuronide & $17.1 \pm 5.1$ & $0.02 \pm 0.04$ & $0.34 \pm 0.05$ \\
Genistein-7-O-sulfate-4'-O-sulfate & $1.7 \pm 0.4$ & $0.18 \pm 0.03$ & $1.09 \pm 0.41$ \\
Genistein-4'-O-sulfate-7-O-glucuronide-7-O-sulfate & $6.4 \pm 0.6$ & $0.85 \pm 0.12$ & $2.96 \pm 0.79$ \\
Total/mean & 5.4 & $1.58 \pm 0.16$ & $18.34 \pm 2.67(29.5 \%)$ \\
\end{tabular}

Data presented as mean values \pm standard error $(n=10)$. Italicized figures in parentheses represent urinary recoveries as a percentage of intake. Adapted from Hosoda et al. (213).

synthesizing equol, as it $S$-enantiomer, and those who lack the microbes to do so $(225,409)$.

Microbial metabolism of genistein is different to that of daidzein. Genistein is reduced to dihydrogenistein, which is further metabolized to 6'-hydroxy-O-desmethylangolensin (225). In vitro incubations with human fecal and rat cecal microbiota revealed formation of 2-(4'-hydroxyphenyl)propionic acid from 6'-hydroxy-O-desmethylangolensin, indicating $\mathrm{C}$ - ring fission (81) (Fig. 22). Heinonen et al. (186) identified four novel isoflavonoid metabolites from human urine after soy supplementation: $3^{\prime \prime}$-hydroxy-O-desmethylangolensin, $3^{\prime}, 4^{\prime}, 7-$ trihydroxyisoflavanone, $4^{\prime}, 7,8$-trihydroxyisoflavanone, and 4',6,7-trihydroxyisoflavanone (Fig. 23).

Red clover (Trifolium pratense), which contains the isoflavones biochanin A and formononetin, is consumed by cows in Finland, and as a consequence, high levels of equol have<smiles>O=c1c(-c2ccc(O)cc2)coc2cc(O)ccc12</smiles>
Daidzein

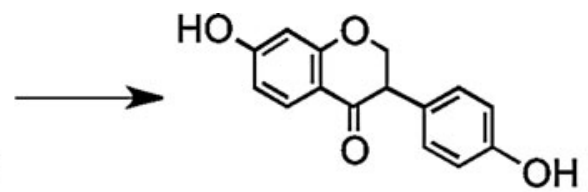

Dihydrodaidzein

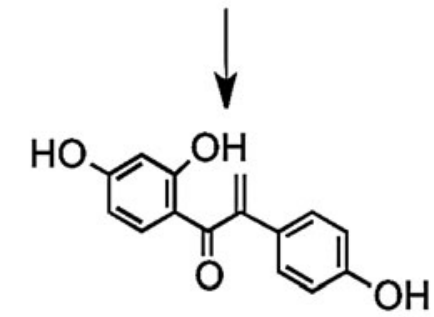

2'-Dehydro-O-desmethylangolensin

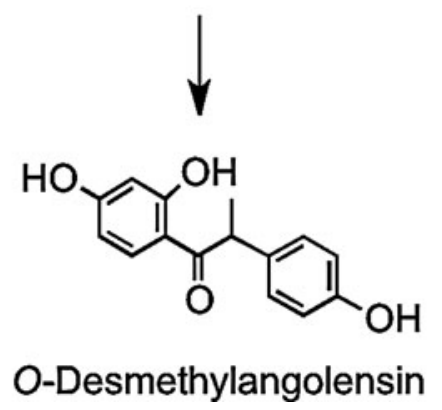<smiles>Oc1ccc(C2COc3cc(O)ccc3C2O)cc1</smiles>

Tetrahydrodaidzein<smiles>Oc1ccc(C2COc3cc(O)ccc3C2)cc1</smiles>

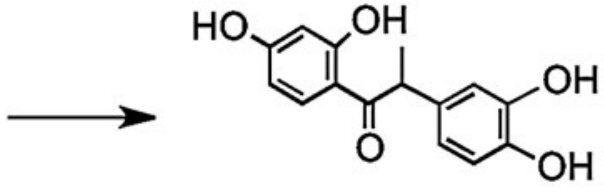

3"-Hydroxy-O-desmethylangolensin

FIG. 21. Microbial metabolism of the isoflavonoid genistein. Adapted from Joannou et al. (225) and Setchell et al. (409). 
<smiles>O=c1c(-c2ccc(O)cc2)coc2cc(O)cc(O)c12</smiles>

Genistein

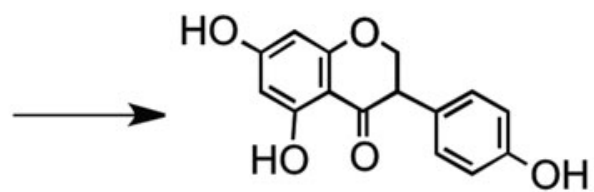

Dihydrogenistein

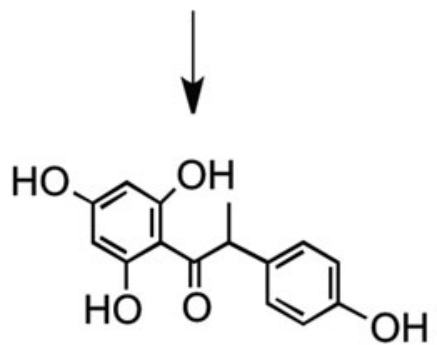

FIG. 22. Microbial metabolism of the isoflavonoid daidzein. Adapted from Coldham et al. (81) and Joannou et al. (225).

\section{6'-Hydroxy-O-desmethylangolensin}<smiles>CC(C)CC(C)C(=O)O</smiles>

\section{2-(4'-Hydroxyphenyl)propionic acid}

been detected in Finnish milk that is sold for human consumption (208). In humans, urinary excretion indicates that formononetin is readily metabolized to daidzein, which can be converted to $\mathrm{O}$-desmethylangolensin and equol, as well as being metabolized to dihydroformononetin and angolensin. Biochanin $\mathrm{A}$ is metabolized to dihydrobiochanin $\mathrm{A}$ and $6^{\prime}$ hydroxyangolensin and is also converted, via genistein, to $6^{\prime}$ hydroxy-O-desmethylangolensin (Fig. 24) (185). This explains the similarity of these microbial metabolites to those of daidzein and genistein (15).

\section{E. Flavan-3-ols}

Bioavailability studies with flavan-3-ols typically involve acute supplementation with either green tea or cocoa-based products. In a recent study, Ottaviani et al. (336) investigated the bioavailability of different enantiomeric forms of flavan3-ol monomers in a study in which adult human males consumed equal quantities of ( -$)$-epicatechin, ( -$)$-catechin, (+)-epicatechin, and (+)-catechin (see Fig. 8) in a cocoa drink. Based on plasma concentrations and urinary excretion, the<smiles>CC(C(=O)c1ccc(O)cc1O)c1ccc(O)c(O)c1</smiles>

\section{3"-Hydroxy-O-desmethylangolensin}<smiles>O=C1c2ccc(O)c(O)c2OCC1c1ccc(O)cc1</smiles>

4',7,8-Trihydroxy-isoflavanone<smiles>O=C1c2cc(O)c(O)cc2OCC1c1ccc(O)cc1</smiles>

\section{4',6,7-Trihydroxy-isoflavanone}<smiles>O=C1c2ccc(O)cc2OCC1c1ccc(O)c(O)c1</smiles>

3',4',7-Trihydroxy-isoflavanone
FIG. 23. Human urinary metabolites excreted after the consumption of soy. Adapted from Heinonen et al. (186) 
<smiles>COc1ccc(-c2coc3cc(O)ccc3c2=O)cc1</smiles>

Formononetin

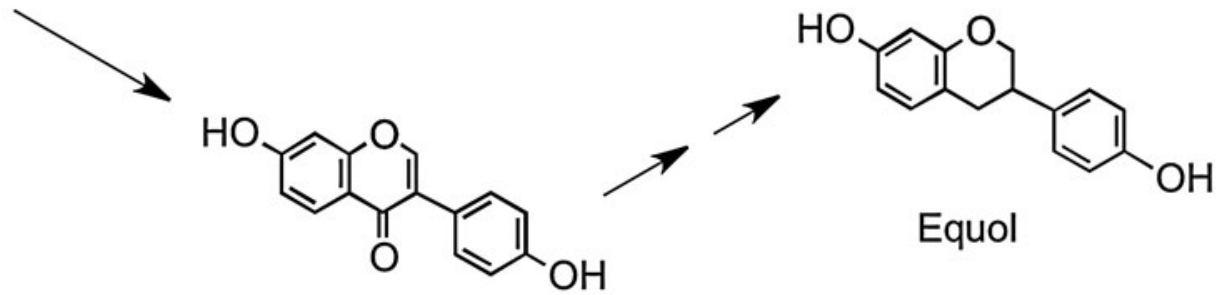

Daidzein

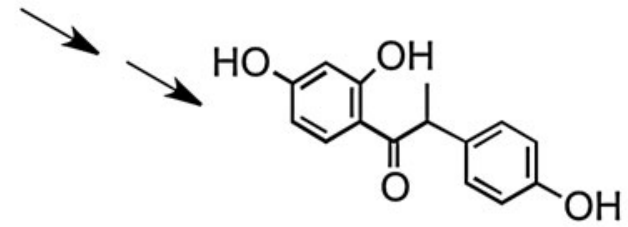

O-Desmethylangolensin

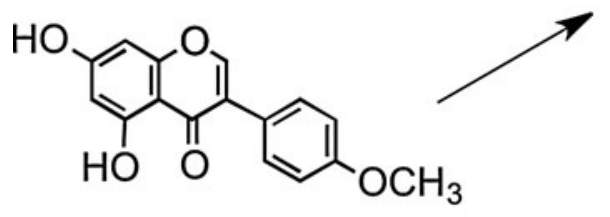<smiles>COc1ccc(C(CCCCCCOc2ccccc2)c2c(O)cc(O)cc2O)cc1</smiles>

Biochanin A

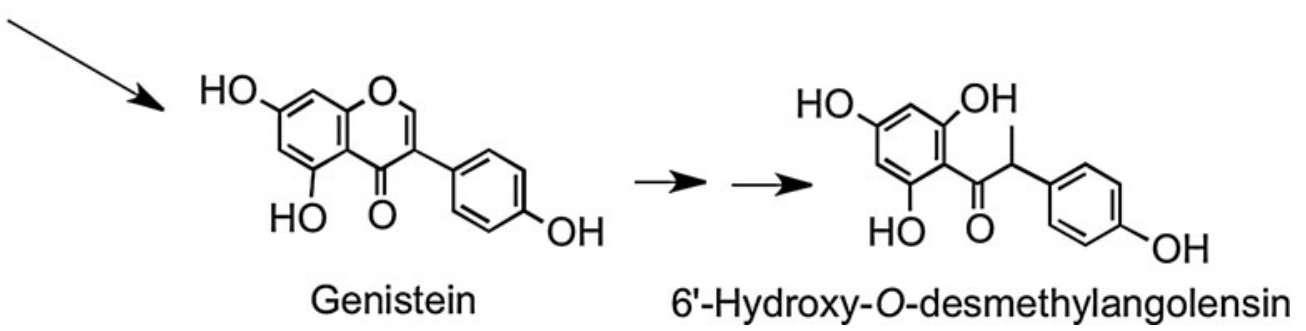

FIG. 24. Human metabolism of the red clover isoflavones formononetin and biochanin A. Adapted from Heinonen et al. (185).

bioavailability of the stereoisomers was ranked as (-)epicatechin $>(+)$-epicatechin $=(+)$-catechin $>(-)$-catechin. There were also differences in the metabolic fate of the catechin and epicatechin epimers as reflected in the ratios of their $3^{\prime}$ - and 4'-O-methylated metabolites. In addition, the levels of nonmethylated metabolites of $(-)$ - and $(+)$ epicatechin in plasma and urine differed, demonstrating that flavan-3-ol stereochemistry also affects metabolic pathways other than $\mathrm{O}$-methylation. The samples were analyzed as aglycones released by glucuronidase/sulfa- tase treatment, so it was not possible to determine in any detail to what degree this impacted on the production of glucuronide and sulfate metabolites. As the individual flavan-3-ol stereoisomers in cocoa products and green teas used in feeding studies are usually not determined, this finding raises the possibility that varying stereochemical ratios could be a contributing factor in the different (epi)catechin metabolite profiles reported in the literature, with the stereochemical variation reflecting differences in product processing. 
1. Cocoa flavan-3-ol monomers. Because the stereochemistry of the flavan-3-ols consumed in cocoa as well as the stereochemistry of the flavan-3-ol metabolites observed in plasma and urine have rarely been characterized to the enantiomer level, this uncertainty will be made clear by using, for example, (epi)catechin and (epi)gallocatechin rather than the apparently more-precise terms used in almost all the original publications.

Early human studies on the postingestion fate of cocoa flavan-3-ols treated plasma and urine samples with $\beta$ glucuronidase/sulfatase and analyzed the released (epi) catechin monomers by reverse-phase $\operatorname{HPLC}(199,372,376$, 482). Richelle et al. (376) showed that after the consumption of $40 \mathrm{~g}$ of dark chocolate containing $282 \mu \mathrm{mol}$ of (-)-epicatechin, the (epi)catechin metabolite levels in plasma rose rapidly and reached a $C_{\max }$ of $355 \mathrm{nM}$ with a $\mathrm{T}_{\max }$ of $2.0 \mathrm{~h}$, characteristic of absorption in the upper GIT rather than the large intestine. With double the chocolate intake, the $\mathrm{C}_{\max }$ increased to $676 \mathrm{nM}$ while the $\mathrm{T}_{\max }$ was extended to $2.6 \mathrm{~h}$, which was attributed to the ad libitum consumption of bread by the volunteers rather than the increased intake of chocolate. Wang et al. (482) also carried out a dose study in which varying amounts of chocolate were served with $40 \mathrm{~g}$ of bread. The data, which are summarized in Table 4, show a positive relationship between intake and (epi)catechin plasma concentrations.

In a further study, Baba et al. (20) fed a chocolate containing $760 \mu \mathrm{mol}$ of (-)-epicatechin and $214 \mu \mathrm{mol}$ of catechin, most probably the (-)-isomer, to human subjects and collected plasma and urine over the ensuing $24-\mathrm{h}$ period. The combined $\mathrm{C}_{\max }$ of the metabolites was $4.8 \pm 0.9 \mu \mathrm{M}$, and the $\mathrm{T}_{\max }$ was $2 \mathrm{~h}$. The total 0-24-h urinary excretion of the (epi)catechin and methyl-(epi)catechin metabolites was $227 \pm 39 \mu \mathrm{mol}$, which corresponds to $29.9 \%$ of the ingested (-)-epicatechin. As will be discussed later, this high level of excretion of (epi)catechin metabolites has been confirmed in a number of subsequent feeding studies with both cocoa and green tea.

More recent cocoa flavan-3-ol bioavailability studies have analyzed plasma and urine samples using the HPLC-MS ${ }^{2}$ methodology. In one such study by Mullen et al. (320), volunteers drank a cocoa beverage containing $22.3 \mu \mathrm{mol}$ of catechin, almost all of it as the less-bioavailable (-)-isomer, and $23.0 \mu \mathrm{mol}$ of (-)-epicatechin. Two flavan-3-ol metabolites were detected in plasma, an $\mathrm{O}$-methyl-(epi)catechin-O-sulfate and an (epi)catechin- $O$-sulfate. Both had a $\mathrm{C}_{\max }$ below $100 \mathrm{nM}$ and a $\mathrm{T}_{\max }$ of $<1.5 \mathrm{~h}$, and after $8 \mathrm{~h}$, only trace levels remained in the circulatory system. The two sulfated flavan-3-ols were

Table 4. Concentration of (Epi)catechin Metabolites in Plasma of Volunteers 0, 2, ANd 6 H After the

Ingestion of Chocolate Containing 159, 312, AND $417 \mu \mathrm{MOL}$ OF $(-)$-EPICATECHIN

\begin{tabular}{lccc}
\hline $\begin{array}{l}(-)-E p i c a t e c h i n \\
\text { intake }(\mu \mathrm{mol})\end{array}$ & $0 \mathrm{~h}$ & $2 \mathrm{~h}$ & $6 \mathrm{~h}$ \\
\hline 0 & $1 \pm 1^{\mathrm{a}}$ & $19 \pm 14^{\mathrm{a}}$ & $1 \pm 1^{\mathrm{a}}$ \\
159 & $2 \pm 2^{\mathrm{a}}$ & $133 \pm 27^{\mathrm{c}}$ & $26 \pm 8^{\mathrm{b}}$ \\
312 & $4 \pm 2^{\mathrm{a}}$ & $258 \pm 29^{\mathrm{c}}$ & $66 \pm 8^{\mathrm{b}}$ \\
471 & $4 \pm 3^{\mathrm{a}}$ & $355 \pm 49^{\mathrm{c}}$ & $103 \pm 16^{\mathrm{b}}$
\end{tabular}

Data expressed as mean values in $\mathrm{n} M \pm$ standard error $(n=9-13)$. Mean values with a different superscript are significantly different $(p<0.05)$.

Adapted from Wang et al. (482). also the main metabolites in urine which, in addition, contained an (epi)catechin-O-glucuronide, and a further (epi)catechin-O-sulfate. The amount of flavan-3-ol metabolites excreted in urine over the $0-24$-h collection period was $7.32 \pm 0.82 \mu \mathrm{mol}$, which is equivalent to $16.3 \% \pm 1.8 \%$ of intake. Considering that half of the flavan-3-ol monomer content of the cocoa was (-)-catechin, which has reduced bioavailability, the real figure for (-)-epicatechin absorption was probably nearer $30 \%$, and as such is comparable with urinary (epi)catechin excretion levels reported by Baba et al. (20).

2. Green tea flavan-3-ol monomers. Green tea contains high concentrations of flavan-3-ol monomers. In addition to (-)-epicatechin and (+)-catechin, (epi)gallocatechins and 3$O$-galloylated flavan-3-ols are present, components that do not occur in cocoa. Typically, (-)-epigallocatechin-3-O-gallate, (-)-epigallocatechin, and (-)-epicatechin (Figs. 8 and 9) predominate (110).

In an acute feeding study, healthy human subjects consumed $500 \mathrm{ml}$ of green tea containing $648 \mu \mathrm{mol}$ of flavan-3-ols, after which plasma and urine collected over a 24-h period were analyzed by HPLC-MS ${ }^{3}$ (440). The plasma contained a total of 12 metabolites in the form of $O$-methylated, sulfated, and glucuronide conjugates of (epi)catechin and (epi) gallocatechin along with the native green tea flavan-3-ols (-)-epigallocatechin-3-O-gallate and (-)-epicatechin-3-Ogallate. An analysis of the pharmacokinetic profiles of these compounds is presented in Table 5. None of the flavan-3-ols were present in the circulatory system at $0 \mathrm{~h}$, but they did appear in detectable quantities $30 \mathrm{~min}$ after green tea consumption. The main component that accumulated was an (epi)gallocatechin-O-glucuronide, with a $\mathrm{C}_{\max }$ of $126 \mathrm{nM}$ and a $\mathrm{T}_{\max }$ of $2.2 \mathrm{~h}$, whereas an (epi)catechin-O-glucuronide attained a $\mathrm{C}_{\max }$ of $29 \mathrm{nM}$ with a $1.7 \mathrm{~h} \mathrm{~T}_{\max }$. The unmetabolized flavan-3-ols (-)-epigallocatechin-3-O-gallate and (-)-epicatechin-3-O-gallate had $\mathrm{C}_{\max }$ values of 55 and $25 \mathrm{nM}$ after 1.6 and $2.3 \mathrm{~h}$, respectively. The $\mathrm{T}_{\max }$ durations ranged from 1.6 to $2.3 \mathrm{~h}$ (Table 5); all the flavan-3-ols and their metabolites were present in only trace quantities after $8 \mathrm{~h}$, and were not detected in the 24 -h plasma. These $T_{\max }$ values and the pharmacokinetic profiles are indicative of absorption in the small intestine.

Table 5. Pharmacokinetic Analysis of Flavan-3-ols and Their Metabolites Detected in Plasma of Volunteers Following the Ingestion of 500 ML OF GREen TEA

\begin{tabular}{lcc}
\hline Flavan-3-ols (number of isomers) & $C_{\max }(n \mathrm{M})$ & $T_{\max }(h)$ \\
\hline (Epi)catechin-O-glucuronide (1) & $29 \pm 4.7$ & $1.7 \pm 0.2$ \\
(Epi)catechin-O-sulfates (2) & $89 \pm 15$ & $1.6 \pm 0.2$ \\
O-Methyl-(epi)catechin-O- & $90 \pm 15$ & $1.7 \pm 0.2$ \\
$\quad$ sulfates (5) & & \\
(Epi)gallocatechin-O-glucuronide (1) & $126 \pm 19$ & $2.2 \pm 0.2$ \\
4'-O-Methyl-(epi)gallocatechin- & $46 \pm 6.3$ & $2.3 \pm 0.3$ \\
$\quad$ O-glucuronide (1) & $79 \pm 12$ & $2.2 \pm 0.2$ \\
4'-O-Methyl-(epi)gallocatechin- & & \\
O-sulfates (2) & $55 \pm 12$ & $1.9 \pm 0.1$ \\
(-)-Epigallocatechin-3-O-gallate (1) & $25 \pm 3.0$ & $1.6 \pm 0.2$ \\
(-)-Epicatechin-3-O-gallate (1) & &
\end{tabular}

Data expressed as mean values \pm standard error $(n=10)$.

Adapted from Stalmach et al. (440). 
Table 6. Quantification of the Major Groups of Flavan-3-ol Metabolites Excreted in Urine 0-24 H After the Ingestion of $500 \mathrm{ML}$ of Green TeA BY VOLUNTEERS

\begin{tabular}{lc}
\hline $\begin{array}{l}\text { Flavan-3-ol metabolites } \\
\text { (number of isomers) }\end{array}$ & $\begin{array}{c}0-24 \mathrm{~h} \text { excretion } \\
(\mu \mathrm{mol})\end{array}$ \\
\hline $\begin{array}{l}\text { (Epi)gallocatechin-O-glucuronide (1) } \\
\text { 4'-O-Methyl-(epi)gallocatechin- }\end{array}$ & $6.5 \pm 1.2$ \\
O-glucuronide (1) & $4.4 \pm 1.5$ \\
4'-O-Methyl-(epi)gallocatechin- & $19.8 \pm 0.3$ \\
O-sulfates (2) & \\
(Epi)gallocatechin-O-sulfates (3) & $2.6 \pm 3.0$ \\
Total (epi)gallocatechin metabolites & $33.3(11.4 \%)$ \\
(Epi)catechin-O-glucuronide (1) & $1.5 \pm 0.3$ \\
(Epi)catechin-O-sulfates (2) & $6.7 \pm 0.7$ \\
O-Methyl-(epi)catechin-O-sulfates (5) & $10.9 \pm 1.2$ \\
Total (epi)catechin metabolites & $19.1(28.5 \%)$ \\
Total flavan-3-ol metabolites & $52.4(8.1 \%)$ \\
\hline
\end{tabular}

Data expressed as mean value \pm standard error $(n=10)$. Italicized figures in parentheses indicate the amount excreted as a percentage of intake.

Adapted from Stalmach et al. (440).

The appearance of unmetabolized flavonoids in plasma is unusual. The passage of (-)-epicatechin-3-O-gallate and (-)epigallocatechin-3-O-gallate through the wall of the small intestine into the circulatory system without metabolism could be a consequence of the presence of the 3-O-galloyl moiety interfering with phase II metabolism. Gallic acid per se is readily absorbed with a reported urinary excretion of $37 \%$ of intake $(412,413)$, and so the gallate ester might exhibit improved absorption.

Urine collected $0-24 \mathrm{~h}$ after green tea ingestion contained flavan-3-ol metabolites similar to those detected in plasma, except for the presence of minor amounts of three additional (epi)gallocatechin-O-sulfates and the absence of (-)-epicatechin-3-O-gallate and (-)-epigallocatechin-3-O-gallate (Table 6) (440). There were, however, differences in the relative amount of individual metabolites in plasma and urine. For instance, an (epi)gallocatechin-O-glucuronide was the main metabolite in plasma (Table 5), but not in urine (Table 6). In total, $52.4 \mu \mathrm{mol}$ of metabolites was excreted, which was equivalent to $8.1 \%$ of the ingested green tea flavan-3-ols. When the urinary (epi)gallocatechin and (epi)catechin metabolites were considered separately, a somewhat different picture emerged. The $33.3 \mu \mathrm{mol}$ excretion of (epi)gallocatechin metabolites was $11.4 \%$ of the ingested (-)-epigallocatechin and (+)-gallocatechin, while the $19.1 \pm 2.2 \mu \mathrm{mol}$ recovery of (epi)catechin represented $28.5 \%$ of intake (Table 6$)$. This is in keeping with high urinary recoveries of (epi)catechin metabolites obtained in the studies with cocoa products (see Section III.E.1), indicating that (-)-epicatechin is highly bioavailable and is absorbed and excreted to a much greater extent than other flavonoids, with the possible exception of isoflavones (see Section III.D).

The absence of detectable amounts of ( - )-epigallocatechin3-O-gallate in urine, despite its presence in plasma, an event observed by several investigators $(75,192,464)$, is difficult to explain. It is possible that the kidneys are unable to remove (-)-epigallocatechin-3-O-gallate from the bloodstream, but if this is the case, there must be other mechanisms that result in
TABle 7. URinary ExCRETION OF Flavan-3-OL Metabolites After the Ingestion of INCREASING Doses of (-)-EPICATECHIN AND ( - )-EPIGALLOCATECHIN in Polyphenon E by Humans with an Ileostomy

\begin{tabular}{lccc}
\hline & $\begin{array}{c}22 \mu \mathrm{mol} \\
\text { dose }\end{array}$ & $\begin{array}{c}55 \mu \mathrm{mol} \\
\text { dose }\end{array}$ & $\begin{array}{c}165 \mu \mathrm{mol} \\
\text { dose }\end{array}$ \\
\hline Glucuronides & $1.8(17 \%)$ & $1.0(18 \%)$ & $1.7(19 \%)$ \\
Sulfates & $3.9(37 \%)$ & $2.0(36 \%)$ & $3.6(40 \%)$ \\
Methylated & $4.8(46 \%)$ & $2.6(46 \%)$ & $4.7(51 \%)$ \\
Total (epi)gallocatechin & $5.7 \pm 1.9^{\mathrm{a}}$ & $3.0 \pm 0.8^{\mathrm{a}}$ & $5.3 \pm 1.2^{\mathrm{a}}$ \\
$\quad$ metabolites & $77 \mu \mathrm{mol}$ & $192 \mu \mathrm{mol}$ & $577 \mu \mathrm{mol}$ \\
& dose & dose & dose \\
& $3.4(7 \%)$ & $14(9 \%)$ & $38(10 \%)$ \\
Glucuronides & $33(64 \%)$ & $93(58 \%)$ & $224(59 \%)$ \\
Sulfates & $15(29 \%)$ & $53(33 \%)$ & $120(31 \%)$ \\
Methylated & $36 \pm 9^{\mathrm{a}}$ & $107 \pm 27^{\mathrm{b}}$ & $262 \pm 26^{\mathrm{c}}$ \\
Total (epi)catechin & & & \\
$\quad$ metabolites & & &
\end{tabular}

Values for total (epi)gallocatechin and (epi)catechin metabolites with different superscripts are significantly different $(p<0.05)$.

Metabolites excreted over a 24-h period after ingestion expressed as $\mu \mathrm{mol}$, and italicized figures in parentheses represent the percentage of glucuronide, sulfate, and methylated metabolites.

Adapted from Auger et al. (14).

its rapid decline after reaching $C_{\max }$. Studies with rats have led to speculation that (-)-epigallocatechin-3-O-gallate may be removed from the bloodstream in the liver and returned to the small intestine in the bile $(247,252)$. To what extent enterohepatic recirculation of (-)-epigallocatechin-3-O-gallate, and also (-)-epicatechin-3-O-gallate, occurs in humans remains to be established. Quite possibly, these bile-excreted flavan-3-ols would be degallated by the gut microbiota, and if not more extensively degraded, would be excreted in urine as (epi)catechin and (epi)gallocatechin metabolites. It is of note that feeding studies with $\left[2-{ }^{14} \mathrm{C}\right]$ resveratrol have provided evidence that metabolites of the stilbene do undergo enterohepatic recycling in humans (480).

a. Dose effects. Auger et al. (14) fed ileostomists increasing doses of Polyphenon E, a green tea extract containing a characteristic array of flavan-3-ols; urinary excretion of metabolites was used as a measure of absorption in the small intestine. The data obtained with (epi)gallocatechin and (epi)catechin metabolites are summarized in Table 7. At a dose of $22 \mu \mathrm{mol}$, the $0-24-\mathrm{h}$ excretion of (epi)gallocatechin metabolites was $5.7 \pm 1.9 \mu \mathrm{mol}$, and this figure did not increase significantly with intakes of 55 and $165 \mu \mathrm{mol}$. There is, therefore, a strict limit on the extent to which (epi)gallocatechins can be absorbed. After the ingestion of $77 \mu \mathrm{mol}$ of (epi)catechins, 36 $\pm 9 \mu \mathrm{mol}$ was excreted, and with doses of 192 and $577 \mu \mathrm{mol}$, and urinary excretion increased significantly to $107 \pm 27$ and $262 \pm 26 \mu$ mol. Thus, even the highest dose of (epi)catechins, unlike (epi)gallocatechins, is still readily absorbed. The addition of a 5'-hydroxyl group to (epi)catechin, therefore, markedly reduces the extent to which the molecule can enter the circulatory system from the small intestine. It is also of note that at the three doses that were administered, the ratio of the urinary glucuronide, sulfate, and methylated (epi)catechin metabolites changed little (Table 7), implying that even at the highest intake, the UGT, SULT and COMT 
enzymes involved in the formation of the (epi)catechin metabolites do not become saturated and limit conversions.

b. Colonic catabolism of green tea flavan-3-ols. Stalmach et al. (438) carried out an acute green tea feeding study with ileostomists. The volunteers drank tea containing $634 \mu \mathrm{mol}$ of flavan-3-ols, a very similar intake to that used in their studies with healthy subjects (440). The plasma flavan-3-ol pharmacokinetics were similar to those presented in Table 5, which were obtained from healthy subjects with an intact colon. Urinary excretion by the ileostomists was $8.0 \%$ of intake for (epi)gallocatechin metabolites and $27.4 \%$ of (epi)catechin metabolites, values that were similar to those observed with healthy subjects (see Table 6). This confirms that the flavan-3ol monomers are absorbed in the upper part of the GIT, and suggests that absorption of (epi)catechin and (epi)gallocatechin after degallation of flavan-3-ol gallates excreted in bile does not contribute significantly to bioavailability of the intact flavan-3-ols in consumers with an intact colon. Despite the substantial absorption of green tea flavan-3-ols in the upper GIT, Stalmach et al. (438) found that $69 \%$ of intake was recovered in 0-24-h ileal fluid as a mixture of native compounds and metabolites. Thus, in volunteers with a colon, most of the ingested flavan-3-ols will pass from the small to large intestine, where their fate is a key part of the overall bioavailability equation.

To mimic events taking place in the large intestine, two sets of experiments were carried out by Roowi et al. (387). First, $50 \mu \mathrm{mol}$ of (-)-epicatechin, (-)-epigallocatechin, and (-)epigallocatechin-3-O-gallate was incubated with fecal suspensions in vitro under anaerobic conditions and their degradation to phenolic acid and aromatic catabolites by the microbiota monitored. Secondly, to complement the in vitro incubations, catabolites excreted in urine $0-24 \mathrm{~h}$ after (i) the ingestion of green tea and water by healthy subjects in a crossover study and (ii) the consumption of green tea by ileostomists were also investigated (387).

The data obtained in these studies provided the basis for the operation of the proposed catabolic pathways illustrated in Figure 25. Some of these catabolites, such as 4'-hydroxyphenylacetic acid and hippuric acid ( $N$-benzoylglycine), were detected in urine from subjects with an ileostomy, indicating that they are produced in the body by additional routes unrelated to colonic degradation of flavan-3-ols. It is, for instance, well known that there are pathways to hippuric acid from compounds such as benzoic acid, quinic acid (79), tryptophan, tyrosine, and phenylalanine (50, 171, 408). Nonetheless, the elevated urinary excretion of hippuric acid and 4'-hydroxyphenylacetic acid occurring after green tea consumption is likely to be partially derived from flavan-3-ol degradation.

Quantitative estimates of the extent of ring fission of the flavan-3-ol skeleton are difficult to assess, because as noted above, the production of some of the urinary catabolites is not exclusive to colonic degradation of flavan-3-ols. If these compounds are excluded along with pyrogallol and pyrocatechol, which are derived from cleavage of the gallate moiety from (-)-epigallocatechin-3-O-gallate rather than ring fission, the excretion of the remaining urinary phenolic acids, namely 4hydroxybenzoic acid, 3'-methoxy-4'-hydroxyphenylacetic acid, 3-(3'-hydroxyphenyl)hydracrylic acid, and 5-(3', 4', 5'-trihydroxyphenyl)- $\gamma$-valerolactone, after ingestion of green tea was $210 \mu \mathrm{mol}$ compared to $38 \mu \mathrm{mol}$ after drinking water (387). The $172-\mu \mathrm{mol}$ difference between these figures corresponds to $27 \%$ of the $634 \mu \mathrm{mol}$ of flavan-3-ols present in the ingested green tea (438). Added to this is the $\sim 8 \%$ excretion of glucuronide, sulfate, and methylated flavan-3-ols originating from absorption in the small intestine. This estimate of a $35 \%$ recovery is nonetheless a minimum value, because with the analytical methodology used, some urinary catabolites will have escaped detection (387). This will include glucuronide and sulfate metabolites of 5-( $3^{\prime}, 4^{\prime}, 5^{\prime}-$ trihydroxyphenyl)- $\gamma$-valerolactone, 5-( $3^{\prime}, 4^{\prime}$-dihydroxyphenyl)- $\gamma$ valerolactone, and 5-(3',5'-dihydroxyphenyl)- $\gamma$-valerolactone, which were detected after green tea consumption with a cumulative $0-24-h$ excretion corresponding to $16 \%$ of flavan-3-ol intake $(277,303,400)$. More recently, in a similar study in which urine was collected for $24 \mathrm{~h}$ after green tea intake, valerolactone metabolites were excreted in quantities equivalent to $36 \%$ of intake (108). When added to the $35 \%$ recovery of Roowi et al. (387), this gives a total of $71 \%$ of intake. While this figure is obviously an approximation because of factors such as different volunteers, flavan-3-ol intakes, and analytical methodologies, it does demonstrate that despite substantial modification as they pass through the body, there is a very high urinary recovery of flavan-3-ols, principally in the form of colon-derived catabolites. Moreover, the excretion of colonic valerolactones has been shown to continue far beyond $24 \mathrm{~h}$ after ingestion, as some of these metabolites have been detected in urine after $54 \mathrm{~h}$ (108). This observation is consistent with a possible underestimation of the real bioavailability of flavan-3-ols, at least when green tea is consumed. In this context, the study of Calani et al. (60), who monitored urinary excretion of (epi)catechin catabolites by 20 healthy volunteers for $48 \mathrm{~h}$ after ingestion of green tea, is of interest. On a mole-for-mole basis, some volunteers exhibited a $100 \%$ recovery of flavan-3-ols in urine, whereas with others, excretion was $<30 \%$ of intake. Arguably, this suggests that colonic ring-fission catabolism could be a key factor in the bioactivity of green tea flavan-3-ols.

c. Identification and quantification of flavan-3-ol metabolites. Rather different (epi)catechin metabolite profiles have been obtained by different research groups who in the absence of standards used HPLC-MS ${ }^{3}$ to partially identify metabolites that were then quantified by reference to (-)-epicatechin. In the Mullen et al. (320) cocoa investigation, an (epi)catechin$\mathrm{O}$-sulfate and an O-methyl-(epi)catechin-O-sulfate were detected in plasma. These sulfates were also the main metabolites in urine, which also contained an additional (epi)catechin-O-sulfate and smaller amounts of an (epi)catechin-O-glucuronide. As outlined above, Auger et al. (14) and Stalmach et al. $(438,440)$, using an identical analytical methodology, detected a similar spectrum of (epi)catechin metabolites in plasma and urine collected after ingestion of green tea flavan-3-ols. However, in cocoa studies by other groups in which plasma and urine samples were analyzed by HPLC$\mathrm{MS}^{3}$, an (epi)catechin-O-glucuronide was the main metabolite, and sulfates were either absent or minor components (390-393, 458). The reason for these varying metabolite profiles, especially the dominance of glucuronides in some studies and sulfates in others, could be due to losses of (epi)catechin-O-sulfates that are known to be unstable during sample processing before analysis (106). A further factor influencing quantitative estimates is that the response of the mass spectrometers used by the different groups may vary to an extent, which in the absence of reference compounds 
<smiles>C=CC(C)C(=O)c1cc(O)c(O)c(O)c1</smiles>

(-)-Epigallocatechin-3-O-gallate (IF)<smiles>CCCOc1cccc(O)c1O</smiles>

Pyrogallol (F,U)<smiles>Oc1ccccc1O</smiles>

Pyrocatechol $(F, U)$<smiles>C=C=CCOc1cc(O)cc(O)c1C[C@@H](O)c1cc(O)c(O)c(O)c1</smiles>

(-)-Epigallocatechin (IF)<smiles>CCCOc1cc(O)cc(O)c1CC(O)Cc1cc(O)c(O)c(O)c1</smiles>

1-(3',4',5'-Trihydroxyphenyl)3-(2",4",6"-trihydroxy)propan-2-ol*

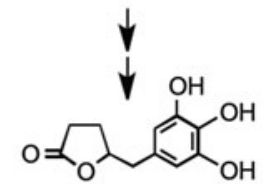

5-(3',4',5'-Trihydroxyphenyl) $-\gamma$-valerolactone $(F, U)$

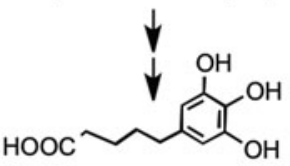

5-(3',4',5'-Trihydroxyphenyl)-valeric acid*<smiles>C=C=CC=Cc1cc(O)cc(O)c1C[C@@H](O)[C@H]1Cc2cc(O)cc(O)c2O1</smiles>

(-)-Epicatechin (IF)<smiles>CC(C)(C)COc1cc(O)cc(O)c1CC(O)Cc1ccc(O)c(O)c1</smiles>

1-(3',4'-Dihydroxyphenyl)3-(2",4",6"-trihydroxy)propan-2-ol*

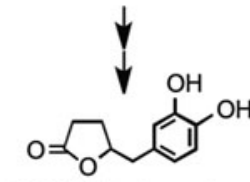

5-(3',4'-Dihydroxyphenyl) $-\gamma$-valerolactone (F)

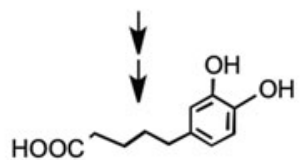

5-(3',4'-Dihydroxyphenyl)-valeric acid (F)
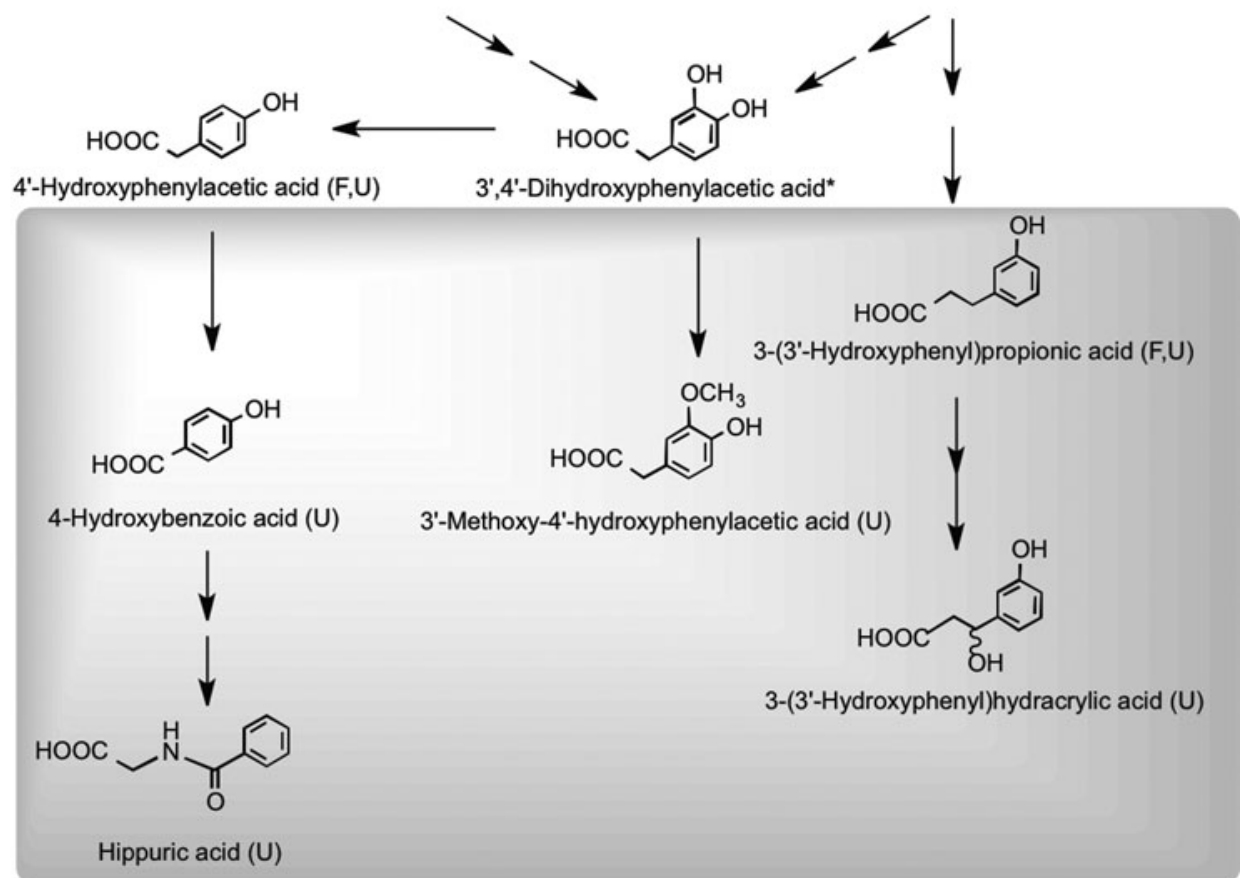

FIG. 25. Proposed pathways involved in the colonic catabolism and urinary excretion of green tea flavan-3-ols. After consumption of green tea, more than $50 \%$ of the ingested flavan-3-ols (gray structures) pass into the large intestine. When incubated with fecal slurries, these compounds are catabolized by the colonic microflora, probably via the proposed pathways illustrated. Analysis of urine after green tea consumption indicates that some of the colonic catabolites enter the circulation and undergo further phase II metabolism before being excreted in urine. Structures in the gray box indicate such catabolites that are detected in urine, but not produced by fecal fermentation of (-)-epicatechin, (-)-epigallocatechin, or $(-)$ epigallocatechin-3-O-gallate. The dotted arrow between pyrogallol and pyrocatechol indicates that this is a minor conversion. Double arrows indicate conversions where the intermediates did not accumulate and are unknown. Compounds detected in ileal fluid after green tea consumption (IF); catabolites detected in fecal slurries (F) and in urine (U); potential intermediates that did not accumulate in detectable quantities in fecal slurries $\left(^{*}\right)$. Adapted from Roowi et al. (387). 


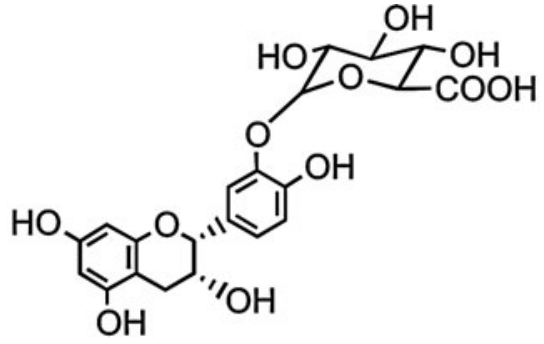

(-)-Epicatechin-3'-O-glucuronide<smiles>COc1ccc([C@H]2Oc3cc(OC4(O)OC(CO)C(O)C(O)C(O)C4O)cc(O)c3C[C@H]2O)cc1O</smiles>

4'-O-Methyl-(-)-epicatechin-7-O-glucuronide<smiles>O=S(=O)([O-])Oc1cc([C@@H]2Oc3cc(O)cc(O)c3C[C@H](O)O2)ccc1O</smiles>

(-)-Epicatechin-3'-O-sulfate<smiles>O=S(=O)(O)c1cc(O)cc2c1C[C@H](O)[C@H](c1ccc(O)c(O)c1)O2</smiles>

(-)-Epicatechin-5-O-sulfate<smiles></smiles>

(-)-Epicatechin-7-O-sulfate

FIG. 26. Human (-)-epicatechin plasma metabolites detected in plasma after the consumption of an-(-)-epicatechinrich cocoa drink. Adapted from Ottaviani et al. (337).

cannot be determined. Thus, it is possible that the Mullen et al. (320), Auger et al. (14), and Stalmach et al. $(438,440)$ studies, and others by this group $(42,435)$, may have used instruments that are more sensitive to sulfates than to glucuronides, and vice versa for other investigators. One way around this is to treat samples with sulfatase/glucuronidase to release the aglycones, but this too has its drawbacks, as different batches of the enzyme preparations vary in the effectiveness with which they hydrolyze flavan-3-ol sulfate metabolites (337).

In a recent human cocoa feeding study in which (i) authentic flavan-3-ol metabolites were available to assist analysis, and (ii) the (-)-epicatechin intake was $6.2 \mathrm{mmol} / \mathrm{kg}$ body weight, the following metabolites were identified in plasma with a $\mathrm{T}_{\max }$ of $2 \mathrm{~h}$ : (-)-epicatechin-3'-O-glucuronide $\left(\mathrm{C}_{\max }, 589 \pm 85 \mathrm{nM}\right)$, 4'-O-methyl-(-)-epicatechin-7-O-glucuronide $\left(\mathrm{C}_{\max }, \sim 20 \mathrm{nM}\right)$, (-)-epicatechin-3'-O-sulfate $\left(\mathrm{C}_{\max }\right.$, $331 \pm 26 \mathrm{nM})$, ( - )-epicatechin-5-O-sulfate $\left(\mathrm{C}_{\max }, 37 \pm 3 \mathrm{nM}\right)$, and (-)-epicatechin-7-O-sulfate $\left(\mathrm{C}_{\max }, 12 \pm 1 \mathrm{nM}\right)$ (Fig. 26). Also present in trace amounts were four distinct $3^{\prime}$ - and $4^{\prime}-O$ methyl-(-)-epicatechin-O-sulfates, each of which was sulfated at the either C-5 or C-7 position (337). This establishes that the main (-)-epicatechin metabolite in humans is (-)-
epicatechin-3'-O-glucuronide, and that sulfate metabolites accumulate in plasma at slightly lower concentrations.

3. Black tea theaflavins and thearubigins. Although consumed far more extensively in Europe than green tea, the absorption of flavan-3-ols from black tea, their gut microbial catabolism, and human metabolism have been investigated less extensively, and such studies as have been performed focused on the absorption of flavan-3-ol monomers (192). The appearance of the gallic acid metabolites 3-O-methylgallic acid, 4-O-methylgallic acid, and 3,4-di-O-methylgallic acid in urine (Fig. 27) has also been reported and used as an index of black tea consumption $(201,202)$, although these metabolites are also to be expected after not only green and black tea consumption but also the ingestion of certain fruits, such as grapes, and the associated wines. The absorption and metabolism of flavan-3ol monomers from black tea are not obviously different from those observed after green tea consumption, although pro rata, the dose of flavan-3-ols is much reduced.

To date, only one study has investigated the absorption of mixed theaflavins (theaflavin 17.7\%, theaflavin-3-O-gallate $31.8 \%$, theaflavin- $3^{\prime}-O$-gallate $16.7 \%$, and theaflavin-3, $3^{\prime}-$<smiles>COc1cc(C(=O)O)cc(O)c1O</smiles>

3-O-Methylgallic acid<smiles>COc1c(O)cc(C(=O)O)cc1O</smiles>

4-O-Methylgallic acid<smiles>COc1cc(C(=O)O)cc(O)c1OC</smiles>

3,4-O-Dimethylgallic acid

FIG. 27. Methylated gallic acid derivatives excreted after the consumption of black tea. 
digallate 31.4\%) (Fig. 9) (318). An extremely high-dose $700 \mathrm{mg}$ mixed theaflavins, equivalent to about 30 cups of black tea, was given to two healthy volunteers, one male and one female. Plasma and urine concentrations were analyzed by HPLC-MS ${ }^{2}$ after enzymatic deconjugation with $\beta$ glucuronidase and sulfatase. Only theaflavin was detected because the enzyme treatment also removed ester gallate. Maximum theaflavin concentrations detected in the plasma of the female and male volunteers were 1.8 and $0.9 \mathrm{fM}$, respectively, and maximum urine concentrations were 1.1 and $7.4 \mathrm{fM}$, respectively, all at $2 \mathrm{~h}$. These values should be doubled to correct for the relatively poor recovery observed with standard theaflavin, but even so, the total amount of theaflavin excreted was considerably $<0.001 \%$ of the very large dose consumed (318).

Feeding studies have demonstrated that the consumption of black tea beverage results in a substantially increased excretion of hippuric acid relative to baseline, suggesting that a combination of gut microbial catabolism and postabsorption metabolism results in a significant production of benzoic acid $(79,107,317)$. The yield of benzoic acid excreted as hippuric acid is such that it points to thearubigins and theaflavins serving as substrates in vivo, and being degraded to aromatic acids. The yield of hippuric acid was not significantly affected by the presence of caffeine in the black tea or by the addition of milk, but varied by $\sim 4$-fold between individuals for a given intake of black tea (79).

\section{F. Proanthocyanidins}

There have been numerous feeding studies with animals and humans, indicating that the oligomeric and polymeric proanthocyanidins are not absorbed to any degree. A study in which ileostomists consumed apple juice indicates that most pass unaltered to the large intestine (233), where they are catabolized by the colonic microbiota, yielding a diversity of phenolic acids and aromatic components $(133,291)$, including 3-(3'-hydroxyphenyl)propionic acid and 4-O-methyl-gallic acid $(111,156)$, which are absorbed into the circulatory system and excreted in the urine. There is a report based on data obtained from an in vitro model of gastrointestinal conditions that procyanidins degrade, yielding more readily absorbable flavan-3-ol monomers (234). However, in vivo studies $(119,380,462)$, including feeds to ileostomists (233), have not supported this conclusion, suggesting that no more than $\sim 10 \%$ of procyanidin dimers are converted to monomers in this way $(10,445)$.

There is a report of minor quantities of procyanidin $\mathrm{B}_{2}$ being detected in enzyme-treated human plasma collected after the consumption of cocoa (210). The $\mathrm{T}_{\max }$ and pharmacokinetic profile of the $B_{2}$ dimer were similar to those of flavan-3ol monomers, but the $C_{\max }$ was $\sim 100$-fold lower. Urpi-Sarda et al. (465) also detected and quantified procyanidin $B_{2}$ in human and rat urine after cocoa intake.

Recent studies using procyanidin $\mathrm{B}_{2}$ and $\left[{ }^{14} \mathrm{C}\right]$ procyanidin $B_{2}$ have provided information on their in vitro catabolism by the gut microbiota $(10,444,445)$ and rodent pharmacokinetics (446). After oral dosing of rats with a $\left[{ }^{14} \mathrm{C}\right]$ procyanidin $\mathrm{B}_{2}$ dimer, $\sim 60 \%$ of the radioactivity was excreted in urine after $96 \mathrm{~h}$ with the vast majority in a form very different from the intact procyanidin dosed (446). This observation is consistent with the in vitro studies that show extensive catabolism by the gut microbiota. The scission of the interflavan bond represents a minor route, and the dominant products are a series of phenolic acids having one or two phenolic hydroxyls and between one and five aliphatic carbons in the side chain (10, $444,445)$. There were, in addition, some $\mathrm{C}_{6}-\mathrm{C}_{5}$ catabolites with a side chain hydroxyl group and associated lactones and several diaryl-propan-2-ols, most of which are also produced from the flavan-3-ol monomers via the proposed routes illustrated in Figure 25, whereas $3^{\prime}, 4^{\prime}$-dihydroxyphenylacetic acid is derived from cleavage of the C-ring of the upper flavan-3-ol unit. However, the findings of Stoupi et al. (445) also indicate that a feature of flavan-3-ol catabolism is the conversion to $\mathrm{C}_{6}-\mathrm{C}_{5}$ valerolactones and the progressive $\beta$-oxidation to $\mathrm{C}_{6}-\mathrm{C}_{3}$ and $\mathrm{C}_{6}-\mathrm{C}_{1}$ products, broadly in keeping with the (-)-epicatechin catabolism routes illustrated in Figure 28.

The potential biological effects of procyanidins are generally attributed to their more readily absorbed colonic breakdown products, the phenolic acids and valerolactones, although there is a lack of detailed investigation in this area. There is, however, a dissenting view as trace levels of procyanidins, in contrast to (-)-catechin and (+)-epicatechin, inhibit platelet aggregation in vitro and suppress the synthesis of the vasoconstricting peptide endothelin- 1 by cultured endothelial cells (84). Supporting this view is a study in which individual procyanidins were fed to rats after which dimers through to pentamers were detected in the plasma that was extracted with $8 \mathrm{M}$ urea, rather than the more traditional methanol/acetonitrile, which was proposed to have prevented or reversed the binding of procyanidins to plasma proteins (419). The procyanidins were, however, administered by gavage at an extremely high dose, $1 \mathrm{~g} / \mathrm{kg}$ body weight, and it remains to be determined if procyanidins can be similarly detected in urea-extracted plasma after the ingestion by humans of more dietary-relevant quantities in cocoa or chocolate products.

\section{G. Dihydrochalcones}

Human bioavailability studies with dihydrochalcones have been restricted to feeds with apples, apple cider, and rooibos tea (377). After ingestion, the principal components in apples and cider, phloretin glucosides (Fig. 10), undergo cleavage in the small intestine with the released phloretin being subject to glucuronidation before appearing rapidly in the circulatory system as phloretin-2'-O-glucuronide with a $\mathrm{T}_{\max }$ of $<1 \mathrm{~h}$ (Table 8). The short duration of the $T_{\max }$ values and the similar plasma $C_{\max }$ of the glucuronide in healthy subjects and ileostomists after apple cider consumption (294) are all indicative of absorption in the proximal GIT. Overall recoveries of phloretin derivatives in ileal fluid have varied from $47.3 \%$ (175) to $38.5 \%$ (294) and $24.8 \%$ (233). Ileal fluid contained phloretin-2'-O-(2"-O-xylosyl)glucoside, but none of the ingested phloretin-2'-O-glucoside. This implies that the glucoside is more readily cleaved than the xylosyl-glucoside by LPH in the brush border of the small intestine or by CBG in the epithelial cells. Also detected in ileal fluid were the aglycone phloretin, two further phloretin-O-glucuronides, and two phloretin-O-sulfates (294).

In feeds with apple cider and a polyphenol-rich drink, the main dihydrochalcones in urine was phloretin-2'-O-glucuronide with smaller amounts of two additional phloretin- $O$ glucuronides and a phloretin-O-glucuronide- $O$-sulfate. The overall excretion of these metabolites was $\sim 5.0 \%$ of intake in 


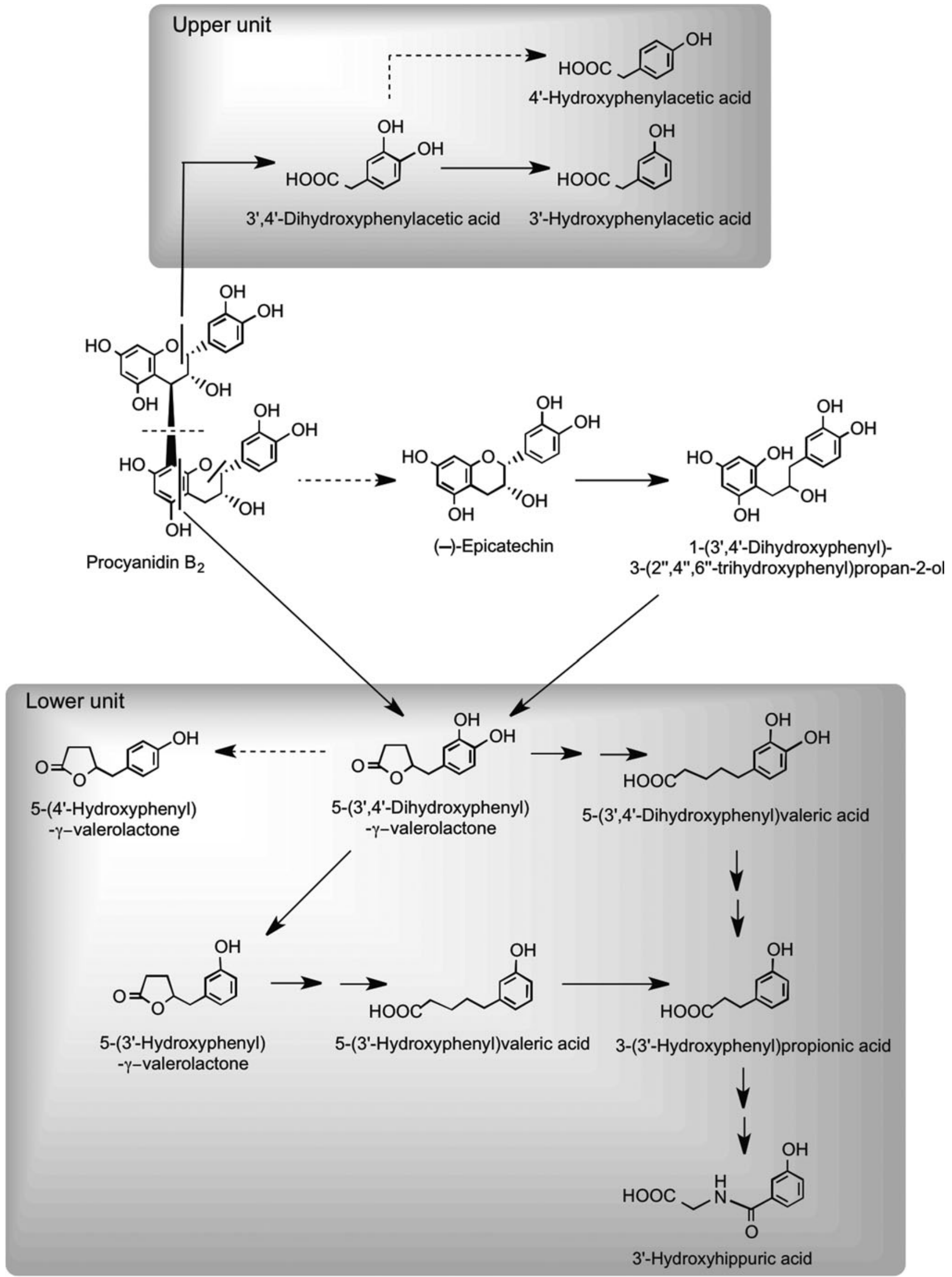

FIG. 28. Proposed pathways for human microbial degradation of procyanidin $\mathbf{B}_{\mathbf{1}}$ dimer. Main routes are indicated with solid arrows, and minor pathways with dotted arrows. Metabolites derived from upper and lower units are grouped within the shaded rectangles. Adapted from Appeldoorn et al. (10) and Stouppi et al. (444, 445). 
Table 8. Plasma Pharmacokinetic Data for Phloretin-2'-O-Glucuronide After the Consumption of Apple Cider and a Polyphenol-Rich Drink by Healthy Volunteers and Subjects WITH AN ILEOSTOMY

\begin{tabular}{lcccc}
\hline & $\begin{array}{c}\text { Intake of } \\
\text { phloretin } \\
\text { derivatives } \\
(\mu \operatorname{mol})\end{array}$ & $\begin{array}{c}\mathrm{C}_{\max } \\
(\mathrm{nM})\end{array}$ & $\begin{array}{c}\mathrm{T}_{\max } \\
(\mathrm{h})\end{array}$ & Reference \\
$\begin{array}{l}\text { Supplement } \\
\text { and volunteers }\end{array}$ & 46 & $73 \pm 11$ & $0.6 \pm 0.1$ & \\
\hline $\begin{array}{l}\text { Apple cider } \\
\text { Healthy volunteers }\end{array}$ & 46 & $93 \pm 29$ & $0.5 \pm 0.0$ & \\
$\quad$ Ileostomists & & & & 42 \\
$\begin{array}{l}\text { Polyphenol-rich drink } \\
\text { Health volunteers }\end{array}$ & 83 & $204 \pm 26$ & $0.6 \pm 0.1$ & \\
\hline
\end{tabular}

both healthy subjects and volunteers with an ileostomy (Table 9), providing further evidence of the absorption of these compounds in the small intestine. Nonetheless, the data obtained with ileal fluid reveal that in subjects with a colon, substantial amounts of ingested phloretin derivatives reach the colon $(42,294)$.

There is only limited information on the fate of dihydrochalcones when they come into contact with the colonic microbiota. Rats fed phloretin excrete the parent dihydrochalcone along with 3-(4'-hydroxyphenyl)propionic acid and 1,3,5-trihydroxybenzene (phloroglucinol). The metabolism and degradation of phloretin- $O$-glycosides after ingestion, therefore, probably follow the pathways outlined in Figure 29 (314). In vitro fecal bacteria convert the synthetic sweetener neohesperidin dihydrochalcone to 3-(3'dihydroxy-4'-methoxyphenyl)propionic acid and 3-(3', 4'hydroxyphenyl)propionic acid with the intermediates hesperetin dihydrochalcone- $4^{\prime}$-O-glucoside and hesperetin dihydrochalcone also being detected (Fig. 30) (45).

As noted in Section II.A.7, the principal components in unfermented rooibos tea are the dihydrochalcone $\mathrm{C}$-glucosides aspalathin and nothofagin (Fig. 10) with smaller amounts of flavanone and flavone $C$-glucosides and flavonol $\mathrm{O}$-glycosides. Fermentation results in a decline in the dihydrochalcones and an increase in the flavanone $\mathrm{C}$-glucosides, while the other flavonoids remained largely unchanged (437).

Table 9. Urinary Excretion of Phloretin Metabolites Over a 24-h Period After the Consumption of Apple Cider and a Polyphenol-Rich Drink by Healthy Volunteers and SubJects With an Ileostomy

\begin{tabular}{lcccc}
\hline & $\begin{array}{c}\text { Intake of } \\
\text { phloretin }\end{array}$ & Urinary excretion & \\
$\begin{array}{c}\text { Supplement } \\
\text { and volunteers }\end{array}$ & $\begin{array}{c}\text { derivatives } \\
(\mu \mathrm{mol})\end{array}$ & $\mu$ mole & $\begin{array}{c}\text { \% of } \\
\text { intake }\end{array}$ & Reference \\
\hline Apple cider & & & & 294 \\
$\quad$ Healthy volunteers & 46 & $2.3 \pm 0.4$ & $5.0 \%$ & \\
$\quad$ Ileostomists & 46 & $2.5 \pm 0.2$ & $5.5 \%$ & \\
$\begin{array}{l}\text { Polyphenol-rich drink } \\
\quad \text { Health volunteers }\end{array}$ & 83 & $4.0 \pm 0.4$ & $4.9 \%$ & \\
\hline
\end{tabular}

Five-hundred-milliliter volumes of the unfermented and fermented rooibos teas that were analyzed by Stalmach et al. (437) were, in the same study, also fed to volunteers, after which plasma and urine collected at intervals over a 24-h period were analyzed by HPLC-MS ${ }^{3}$. This was the first detailed study in which the bioavailability of flavonoid C-glycosides, as opposed to O-glycosides, was investigated in humans. Presumably, because of their low concentration in the teas and/or their low bioavailability, no dihydrochalcones were detected in plasma, although aspalathin metabolites were present in urine in detectable quantities.

Urine contained glucuronides, sulfates, methyl, methyl sulfates, and methyl glucuronide metabolites of aspalathin. In contrast, no nothofagin metabolites were detected. Most urinary excretion of the aspalathin metabolites occurred during the first $5 \mathrm{~h}$ after intake $(80 \%-90 \%$ of the total amounts excreted), indicative of small intestine absorption. In total, $316 \mathrm{nmol}$ of aspalathin metabolites was excreted after an intake of $90 \mu \mathrm{mol}$ with unfermented tea. This represents a recovery of $0.35 \%$ (Table 10 ). After the ingestion of $8 \mu \mathrm{mol}$ of aspalathin with the fermented tea, there was urinary recovery of $14.3 \mathrm{nmol}$ of metabolites corresponding to $0.18 \%$ of intake. Aspalathin, therefore, has very limited bioavailability.

Incubation of aspalathin with artificial gastric juice for up to $2 \mathrm{~h}$ led to a recovery of $\sim 100 \%$, suggesting that the dihydrochalcone-C-glucoside is not subject to degradation in the stomach. The very low urinary recoveries of aspalathin metabolites and their failure to accumulate in detectable quantities in plasma are probably consequences of the $C$-glucoside moiety not being readily cleaved by either LPH or CBG as the dihydrochalcone passes through the small intestine (437).

Kreuz et al. (256) reported urinary excretion of aspalathin in the form of glucuronidated, methyl glucuronidated, methylated, and free aspalathin, in pigs, after intakes of $15.3 \mathrm{~g}$ of aspalathin for 11 days-a daily intake equivalent to $\sim 400$ times that given as a single dose by Stalmach et al. (437). They reported urinary excretion levels ranging from $0.16 \%$ to $0.87 \%$ in three pigs after 7 days of treatment, which is in broad agreement with the observed level of human urinary excretion. This suggests that limited absorption occurs regardless of the dose ingested.

\section{H. Ellagitannins}

Most studies on the bioavailability of ellagitannins have involved feeding humans pomegranate juice, which contains punicalin and punicalagin (Fig. 11) $(68,407)$. However, the bioavailability of ellagitannins in raspberries (158), strawberries, walnuts, and oak-aged wines has also been investigated (69).

After drinking pomegranate juice containing $318 \mathrm{mg}$ of punicalagins, ellagic acid was detected in plasma with $\mathrm{C}_{\max }$ of $60 \mathrm{nM}$ at a $\mathrm{T}_{\max }$ of $0.98 \mathrm{~h}$, suggesting acid hydrolysis of at least some of the ellagitannins releasing free ellagic acid, which is absorbed directly from the stomach or the proximal small intestine (407). Also detected in the plasma of some, but not all, volunteers, mainly $6 \mathrm{~h}$ after supplementation, were urolithin A, urolithin A-3-O-glucuronide, urolithin B, and methylated urolithin $\mathrm{B}$. Urinary metabolites that began to appear after $12 \mathrm{~h}$ included urolithin A-3-O-glucuronide, urolithin B-3-O-glucuronide, and 3,8-O-dimethylellagic acid-2O-glucuronide (see Fig. 31), and excretion continued for up to 


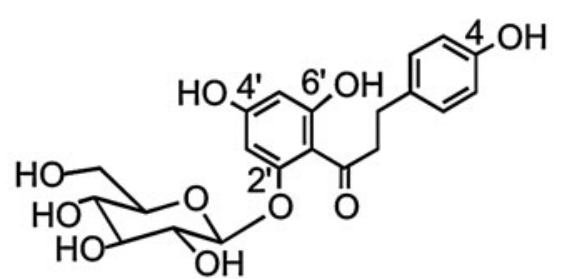

Phloretin-2'-O-glucoside

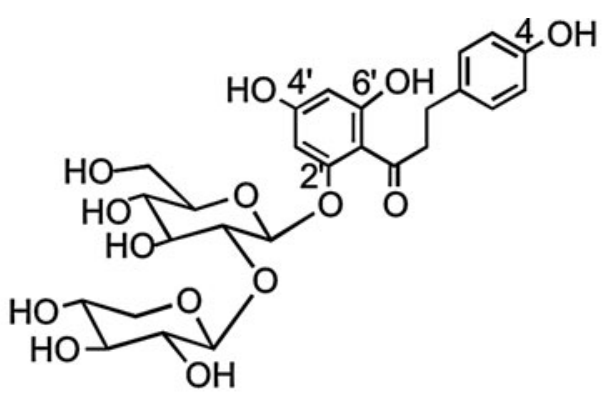

Phloretin-2'-O-(2"-O-xylosyl)glucoside

$L P H, C B G$ or gut microbiota

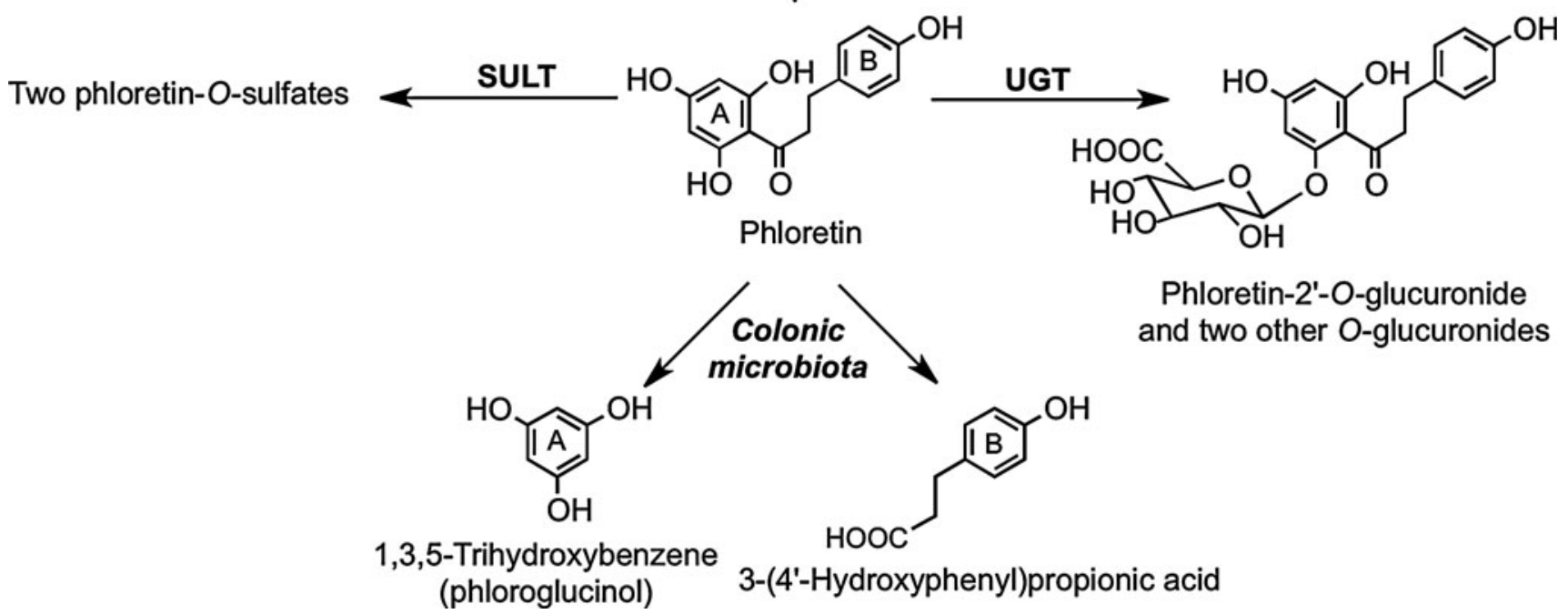

FIG. 29. Metabolism of phloretin-O-glycosides after ingestion. Adapted from Richling (377).

further $36 \mathrm{~h}$. None of these compounds were quantified, and there was much subject-to-subject variation in the spectrum of metabolites produced. This implies that when the ellagitannins and/or ellagic acid reaches the distal part of the small intestine and the colon, they are metabolized by the gut microbiota, producing urolithins $\mathrm{A}$ and $\mathrm{B}$, which are then absorbed along with ellagic acid and subjected to the action of phase II UGTs and/or COMTs before being excreted in urine (407).

In a further study in which volunteers ingested 1 liter of pomegranate juice containing $4.37 \mathrm{~g}$ of punicalagins on a daily basis for 5 days, circulating urolithin levels reached a concentration of $18.6 \mu M$ (68). Feeding human subjects a single dose of strawberries, raspberries, walnuts, and oak-aged red wine, all of which contain ellagitannins, resulted in excretion of urolithin A 3-O-glucuronide in quantities equivalent to $2.8 \%$ (strawberries), 3.4\% (raspberries), 6.5\% (oak-aged red wine), and $16.6 \%$ (walnuts) of intake (69).

After the ingestion of a raspberry supplement, containing $123 \mu \mathrm{mol}$ of ellagitannins, mainly as sanguiin $\mathrm{H}-6$, by healthy subjects, urolithin A-3-O-glucuronide, two of its isomers, and urolithin B-O-glucuronide were excreted in 7-48-h urine (158). There was a marked variation in the urolithin profile of individual volunteers with recoveries relative to the ingested ellagitannins ranging from $0 \%$ to $8.2 \%$, indicative of differences in the colonic microbiota responsible for ellagitannin degradation.

In vitro anaerobic incubations of punicalagin with fecal slurries resulted in the appearance of ellagic acid, urolithin A, isourolithin A, and urolithin B (Fig. 32) (159). There were substantial qualitative and quantitative differences in the profile of metabolites obtained with residual punicalagin concentrations at the end of the 72-h incubation ranging from 1.3 to $27.7 \mu \mathrm{M}$ and the final concentration of urolithins varying from 0 to $27.4 \mu \mathrm{M}$. Once again, this is almost certainly a consequence of person-to-person variations in bacterial composition of the colonic micobiota. Although there was again much sample-to-sample variation, fecal incubations converted ellagic acid to urolithins much more efficiently than punicalagin (Fig. 33). Thus, in vivo, the anaerobic bacterial conversion of ellagitannins to ellagic acid appears to be the rate-limiting step in the production of urolithins in the colon. Furthermore, the fecal incubations did not yield urolithin glucuronides that are excreted in urine after in vivo feeding studies. This points to glucuronidation of urolithins occurring in the wall of the large intestine and/or postabsorption in the liver.

The most detailed study on ellagitannins to date has been carried out with Iberian pigs, which in their natural habit feed 
<smiles>COc1ccc(CC(=O)c2c(O)cc(OC3OC4COC5OC(O)C(O)C(O)C(O)C(O)C5OC3C(O)C4O)cc2O)cc1O</smiles>

Neohesperidin dihydrochalcone<smiles>COc1ccc(CCC(=O)c2c(O)cc(OC3OC(CO)C(O)C(O)C3O)cc2O)cc1O</smiles>

Hesperetin dihydrochalcone-4'-O-glucoside

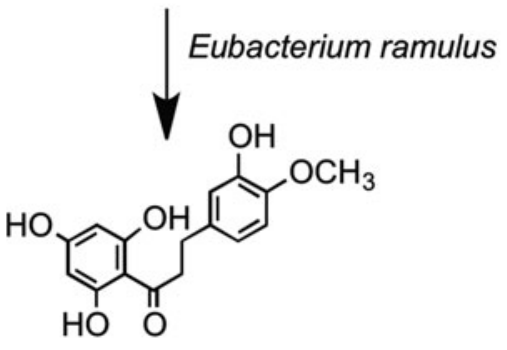

Hesperetin dihydrochalcone

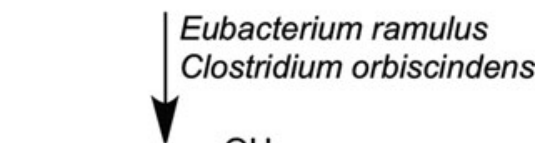<smiles>COc1ccc(CCC(=O)O)cc1O</smiles>

3-(3'-Hydroxy-4'-methoxyphenyl)propionic acid<smiles>CCCCCCCCCCCCCCC(=O)O</smiles>

\section{3-(3',4'-Dihydroxyphenyl)propionic acid}

FIG. 30. Catabolism of neohesperidin dihydrochalcone to 3-(3', $4^{\prime}$-dihydroxyphenyl)propionic acid by human fecal slurries. Steps catalyzed by the colonic bacteria Eubacterium ramulus and Clostridium orbiscindens are indicated. Adapted from Braune et al. (45).

on oak acorns, a rich source of ellagitannins (134). The pigs consumed an average of $4.04 \mathrm{~kg}$ of acorns on a daily basis for 117 days after which tissues and body fluids were processed and analyzed by HPLC-MS ${ }^{3}$. A total of 31 ellagitanninderived metabolites were detected, including 25 urolithin and six ellagic acid derivatives. A summary of the complex picture that emerges is that in the jejunum, the acorn ellagitannins
TABle 10. Summary of the Urinary ExCRETION of Aspalathin Metabolites in Urine Collected From 10 Volunteers After Drinking $500 \mathrm{ML}$ of Unfermented Rooibos Tea Containing $90 \mu \mathrm{MOL}$ of Aspalathin

\begin{tabular}{|c|c|c|c|c|}
\hline \multirow[b]{2}{*}{ Metabolites } & \multicolumn{4}{|c|}{ Quantity excreted in urine (nmol) } \\
\hline & $0-5 h$ & $5-12 h$ & $12-24 h$ & $0-24 h$ \\
\hline $\begin{array}{l}\text { Aspalathin-O- } \\
\text { glucuronide }\end{array}$ & $6.2 \pm 1.1$ & $5.0 \pm 1.1$ & $1.3 \pm 0.6$ & $12 \pm 1.6$ \\
\hline $\begin{array}{l}\text { O-Methyl-aspalathin- } \\
\text { O-glucuronide }\end{array}$ & $180 \pm 22$ & $42 \pm 9.1$ & $7.2 \pm 2.5$ & $229 \pm 26$ \\
\hline Aspalathin-O-sulfate & $40 \pm 6.6$ & $11 \pm 2.3$ & $1.3 \pm 1.1$ & $52 \pm 6.8$ \\
\hline $\begin{array}{l}\text { O-Methyl-aspalathin- } \\
\text { O-sulfate }\end{array}$ & $20 \pm 3.0$ & $2.7 \pm 0.9$ & n.d. & $23 \pm 3.2$ \\
\hline $\begin{array}{l}\text { Total aspalathin } \\
\text { metabolites (\% intake) }\end{array}$ & & & & $0.35 \%$ \\
\hline
\end{tabular}

Data expressed as mean values in $n m o l \pm$ standard error $(n=10)$. Italicized figure in parentheses indicates $0-24-\mathrm{h}$ excretion as a percentage of intake.

Adapted from Stalmach et al. (437).

n.d., not detected.

release ellagic acid (which is metabolized sequentially by intestinal microbiota), producing urolithin $\mathrm{D}$, urolithin $\mathrm{C}$, urolithin A, and urolithin B, as illustrated in Figure 31. These urolithins are absorbed preferentially as their lipophilicity increases with plasma containing mainly urolithin A-3-Oglucuronide and urolithin B-3-O-glucuronide with traces of a urolithin $\mathrm{C}-\mathrm{O}$-glucuronide and 3,8-O-dimethylellagic acid-2$\mathrm{O}$-glucuronide. The urolithin $\mathrm{A}$ and $\mathrm{B}$ glucuronides were the major components in urine. Among the 26 conjugated metabolites were detected in bile were glucuronides and methyl glucuronides of ellagic acid and sizable quantities of urolithin A, C, and D derivatives. This indicates substantial hepatic metabolism and active enterohepatic circulation, and also explains the persistence of urinary urolithin metabolites observed in the human studies. No ellagitannins or their metabolites were detected in body tissues of the Iberian pigs outside the GIT (134). However, after consumption of pomegranate juice and walnuts, urolithin $\mathrm{A}-\mathrm{O}-3$ - glucuronide and traces of urolithin $\mathrm{B}$ have been detected in human prostate tissue (162).

\section{Chlorogenic acids}

Coffee beans as noted in Section II.B is a rich source of chlorogenic acids, mainly 3-O-, 4-O-, and 5-O-caffeoylquinic acids, and smaller amounts of the equivalent feruloylquinic acids, which as a result of their ready extraction by hot water can be consumed in a quantity by regular coffee drinkers (96). The most detailed research on the fate of chlorogenic acids after ingestion of coffee is that of Stalmach et al's. $(436,439)$ in studies with healthy humans and ileostomists, in which it was possible to distinguish between events occurring in the proximal and distal GIT and elucidate the metabolic pathways illustrated in Figure 34. Ileostomists consumed coffee containing $385 \mu \mathrm{mol}$ of chlorogenic acids of which $274 \mu \mathrm{mol}$ was recovered as both the parent compounds and sulfate and glucuronide conjugates in a $0-24$-h ileal fluid. Thus, $\sim 30 \%$ of intake is absorbed in the small intestine, and in healthy 


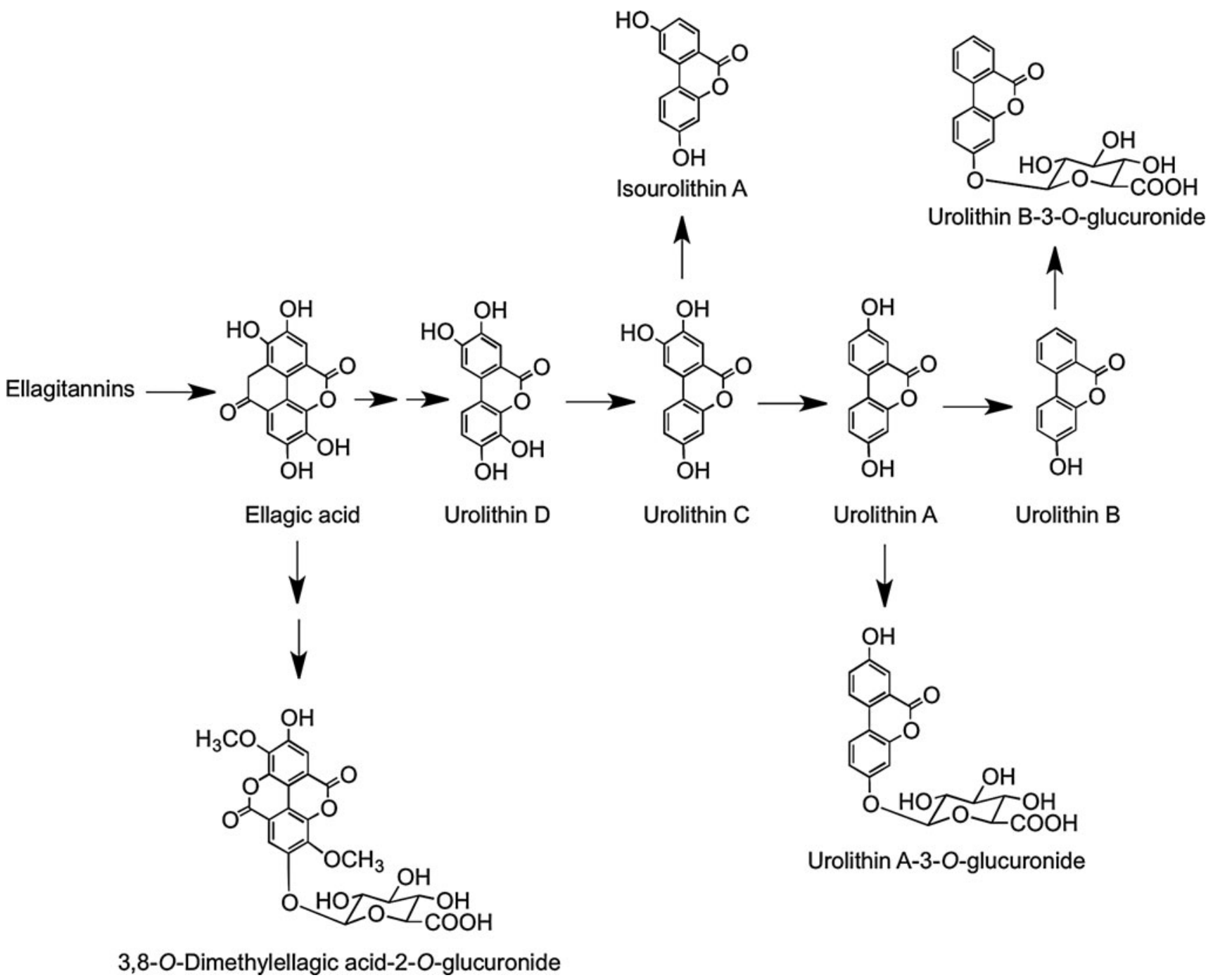

FIG. 31. Potential pathways for the conversion of ellagitannins to urolithins.

subjects with an intact colon, $70 \%$ of the ingested chlorogenic acids pass from the small to large intestine, where they are subject to the action of the colonic microbiota. Urinary excretion, principally of metabolites, by healthy subjects was equivalent to $29.2 \%$ of intake, but only $8.0 \%$ with ileostomists, demonstrating the role of the colon in the bioavailability of chlorogenic acids. The study also showed that urinary dihydrocaffeic acid-3'-O-sulfate and feruloylglycine serve as sensitive biomarkers for the consumption of relatively small amounts of coffee.

Concord grape juice from grapes of $V$. lambrusca contains sizable amounts of hydroxycinnamate tartarate esters with the main component being caftaric acid (Fig. 12). After ingestion, esterases cleave the conjugating tartartic acid, and the released caffeic acid is metabolized and absorbed in a manner similar, but not identical, to that of caffeic acid cleaved from caffeoylquinic acids in coffee $(434,435)$.

\section{J. Resveratrol}

trans-Resveratrol is widely credited with being responsible for the protective effects of red wine in both the press (405) and the scientific literature $(76,399)$. This is almost certainly not true, as the level of the stilbenes in red wines is so low that $>60$ liters would have to be consumed on a daily basis by humans for intake to reach the amounts that are required to increase longevity and provide protective effects in model animal systems (85). Resveratrol is an extremely minor component in the human diet, and as such, its potential use is as a therapeutic agent at pharmacological doses.

Bioavailability studies in humans with trans-resveratrol have shown absorption and a rapid metabolism, but relatively low excretion in urine and feces. Gastric absorption explains the peak of free resveratrol in the plasma $30 \mathrm{~min}$ after ingestion $(8,39,475,479)$. However, the $C_{\max }$ of free resveratrol is typically extremely low: $<37 \mathrm{nM}$ after oral ingestion at physiological doses ranging from 1.1 to $110 \mu \mathrm{mol}(154,479$, 480). In acute feeds with doses of $0.44-22 \mathrm{mmol}$, the higher trans-resveratrol dosage increased the $\mathrm{C}_{\max }$ to $\sim 280 \mathrm{nM}(8,39$, 475). The general consensus is that resveratrol-O-glucuronides and sulfates are the major plasma and urine metabolites, with the sulfates being predominant $(39,40,57,467)$.

Resveratrol-3-O-glucuronide, resveratrol-4'-O-glucuronide, resveratrol-3-O-sulfate, resveratrol-4'-O-sulfate (Fig. 35), a 


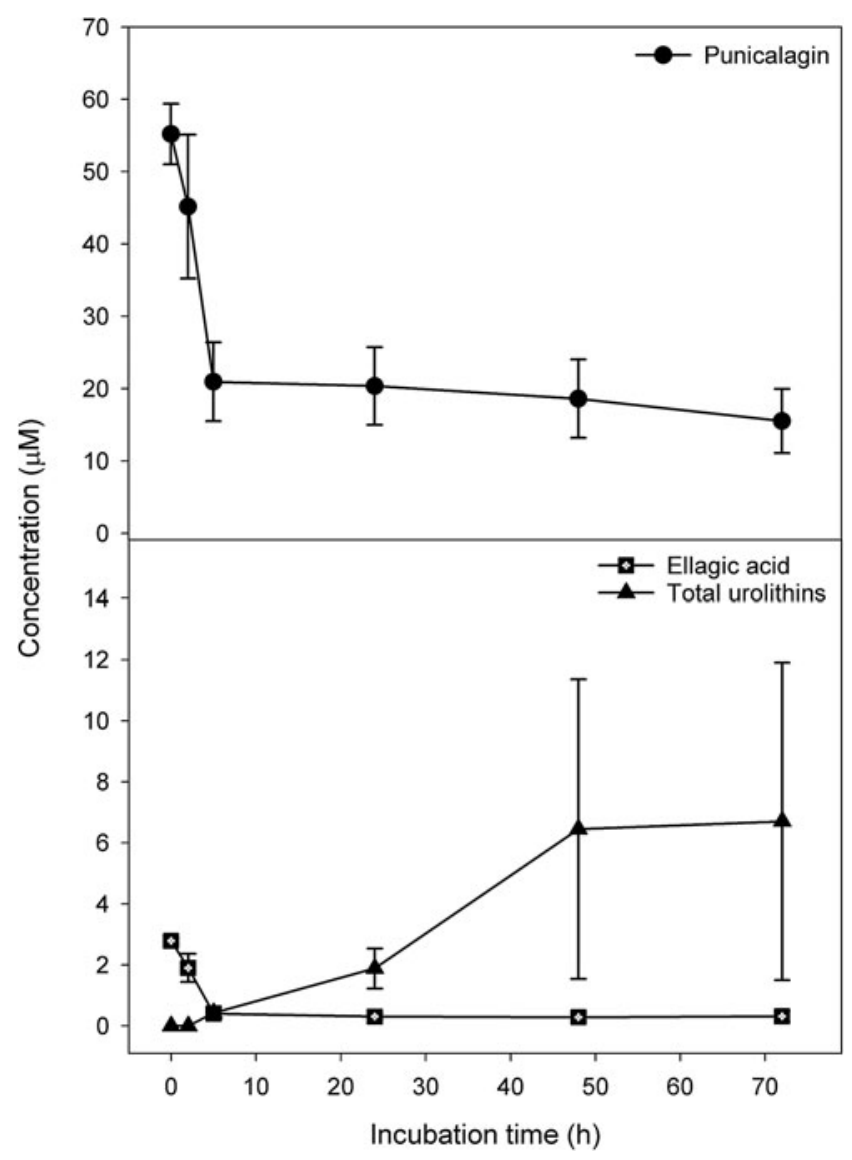

FIG. 32. Anaerobic metabolism of punicalagins to ellagic acid and urolithins in fecal suspensions. Bars represent standard errors $(n=5)$. The initial concentration of punicalagin was $100 \mu \mathrm{M}$. Adapted from Gonzalez-Barrio et al. (159).

resveratrol-O-disulfate, and an $\mathrm{O}$-glucuronide- $\mathrm{O}$-sulfate were found in the plasma of both healthy humans and colorectal cancer patients ingesting 2.2 and $4.4 \mathrm{mmol}$ of trans-resveratrol $(40,348)$. In addition to these metabolites, two novel transresveratrol $\mathrm{C} / \mathrm{O}$-conjugated diglucuronides were detected in plasma and urine of subjects who ingested $128 \mu \mathrm{mol}$ of transpiceid (57).

\section{K. Plant lignans}

A diverse range of plant lignans, including secoisolariciresinol, matairesinol, medioresinol, pinoresinol, and lariciresinol (Fig. 5), are all converted to enterodiol and enterolactone (Fig. 36) by human gut microbiota $(15,128,184$, $308,353,354)$. Enterolactone production in vitro is slow compared with synthesis of enterodiol $(16,128)$. The ratio of enterolactone- and enterodiol-producing bacteria is 1:2000 as shown by culture-based and 16S rRNA-targeted molecular methods. These observations indicate that enterodiolsynthesizing bacteria are dominant, while enterolactoneforming microbiota are a minor population (77). Several research groups have shown a negative association between frequent bowel movements and enterolactone levels (227, 249). Human subjects with more rapid colonic transit may have less time to convert plant lignans to enterolactone than

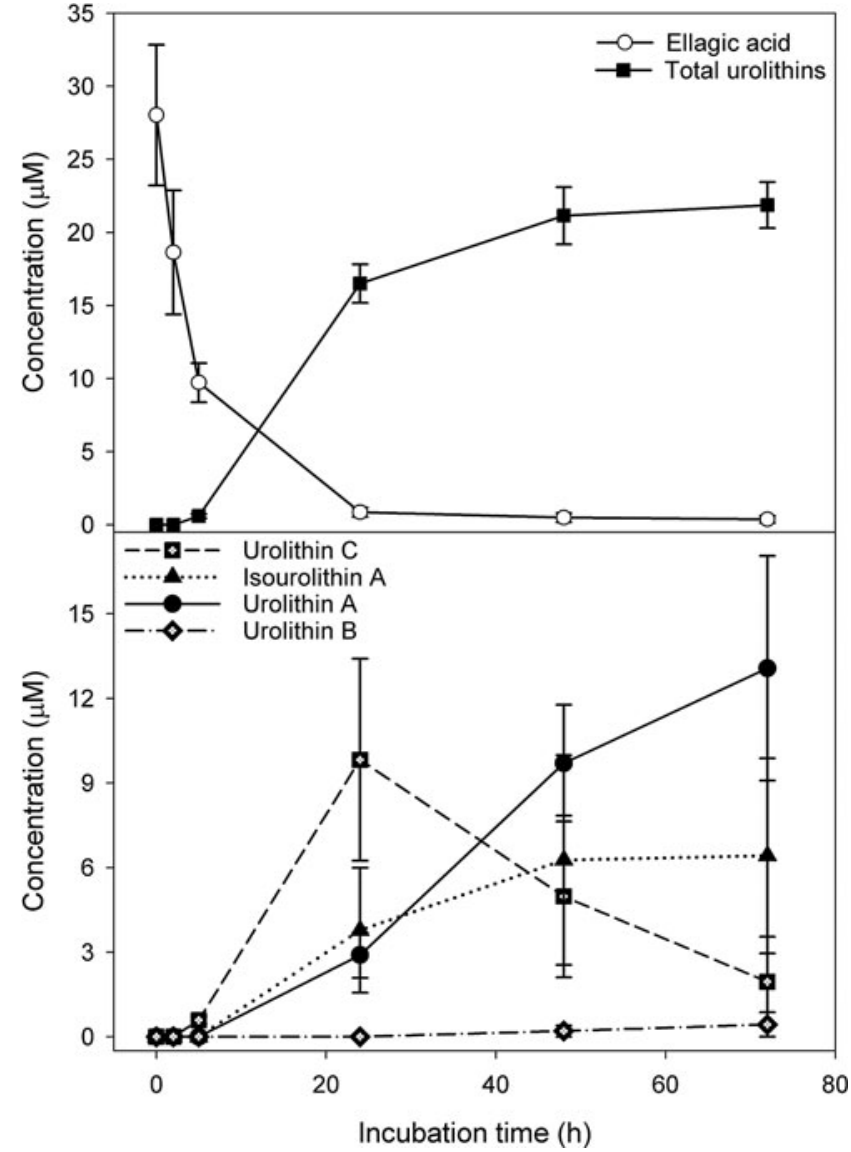

FIG. 33. Anaerobic metabolism of ellagic acid to urolithins in fecal suspensions. Bars represent standard errors $(n=4)$. The initial concentration of ellagic acid was $50 \mu \mathrm{M}$. Adapted from Gonzalez-Barrio et al. (159).

subjects whose colon motility is slow and the subsequent transit time of the colonic contents is more prolong.

\section{Evidence for the Accumulation of (Poly)phenol Metabolites in Body Tissues}

As will be discussed in Section VI, there is evidence from a number of sources that consumption of berry extracts and fruit juices can delay the decline of various aspects of cognitive function in elderly rats and humans (359). There is, however, contradictory evidence as to whether flavonoids themselves cross the blood-brain barrier.

In one of the early studies, Andres-Lacueva et al. (9) detected trace levels of several anthocyanins in brains of rats that had received a diet supplemented with a blueberry extract, containing unspecified amounts of anthocyanins, for a period of 10 weeks. In a further study, $18 \mathrm{~h}$ after feeding pelargonidin to rats by gavage at a dose of $184 \mu \mathrm{mol} / \mathrm{kg}$, the unmetabolized anthocyanidin was detected in brains at a concentration of $c a$. $0.2 \mathrm{nmol} / \mathrm{g}$ (fresh weight) (129). In contrast, anthocyanins did not accumulate in detectable amounts in the brains of rats obtained up to $24 \mathrm{~h}$ after acute supplementation by gavage with $2.8 \mathrm{ml}$ of raspberry juice, which is a nutritional-relevant dose equivalent to a $70-\mathrm{kg}$ human drinking $700 \mathrm{ml}$ of juice (43). In contrast, after a 4-week supplementation of pigs with a 


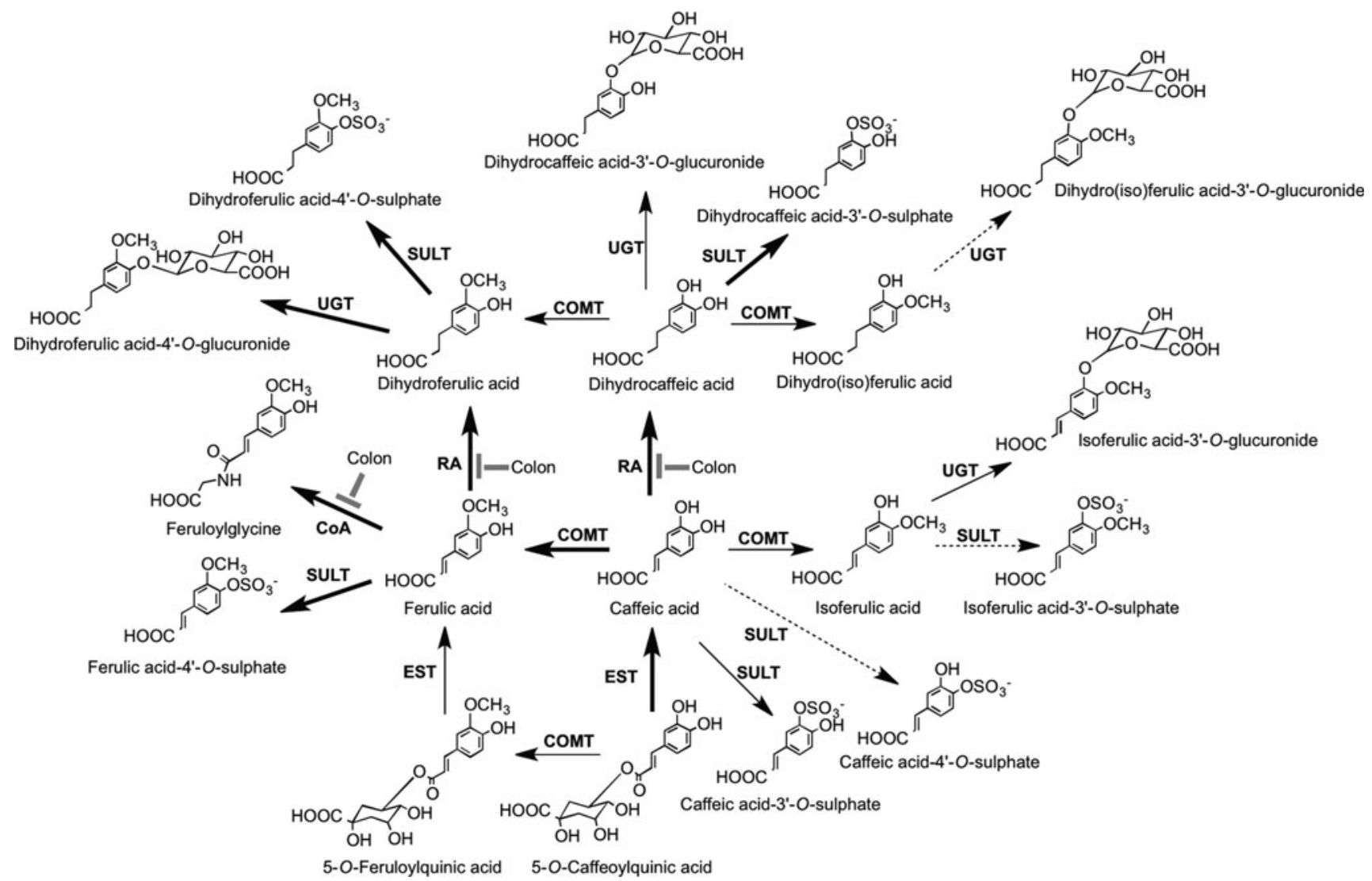

FIG. 34. Proposed metabolism of chlorogenic acids following the ingestion of coffee by volunteers. 5-Caffeoylquinic acid and 5-feruloylquinic acid are illustrated structures, but their respective 3- and 4-isomers would be metabolized in a similar manner. Co-A, Co-enzyme A; EST, esterase; RA, reductase. Bold arrows indicate major routes, and dotted arrows minor pathways. Steps blocked in subjects with an ileostomy, and hence occurring in the colon are indicated. Adapted from Stalmach et al. $(436,439)$.

blueberry extract, containing undefined amounts of anthocyanins, $300 \mathrm{pg}$ of anthocyanins / $\mathrm{g}$ was detected in cerebellum tissue and $700 \mathrm{pg} / \mathrm{g}$ in eye tissue (237). In another study, oral ingestion of $100 \mathrm{mg} / \mathrm{kg}$ of blackcurrant anthocyanins by rats resulted in a plasma anthocyanin $\mathrm{C}_{\max }$ of $1.9 \mu \mathrm{g} / \mathrm{ml} 30 \mathrm{~min}$ after ingestion and a maximum concentration of anthocyanins in the whole eye of $115 \mathrm{ng} / \mathrm{g}$, also after $30 \mathrm{~min}$ (295). However, in a study with male mice, feeding a bilberry (Vaccinium myrtillus) extract for 2 weeks resulted in the accumulation of anthocyanins in detectable amounts in plasma, the liver, kidney, testes, and lungs, but not in other tissues, including the brain and eyes (398). One of the possible reasons for the<smiles>O=C(O)C1(CO)OC(Oc2cc(O)cc(C=Cc3ccc(O)cc3)c2)C(O)C1O</smiles>

trans-Resveratrol-3-O-glucuronide<smiles>O=S(=O)([O-])c1cc(O)cc(/C=C/c2ccc(O)cc2)c1</smiles>

trans-Resveratrol-3-O-sulfate<smiles>O=C(O)C1C(O)OC2(O)OC(Oc3ccc(/C=C/c4cc(O)cc(O)c4)cc3)C1C2(O)O</smiles>

trans-Resveratrol-4'-O-glucuronide<smiles>O=S(=O)(O[Na])Oc1ccc(/C=C/c2cc(O)cc(O)c2)cc1</smiles>

trans-Resveratrol-4'-O-sulfate
FIG. 35. Human metabolites of trans-resveratrol. Adapted from Boocock et al. (40) and Patel et al. (348). 


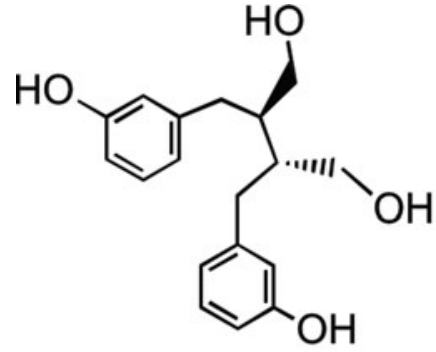

Enterodiol

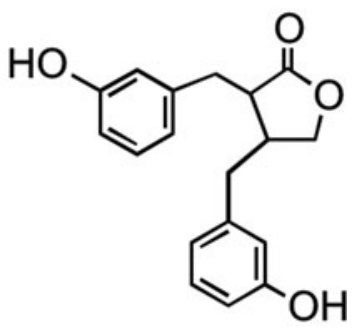

Enterolactone
FIG. 36. The mammalian lignans enterodiol and enterolactone.

seemingly contradictory data obtained in these studies could be the use of extracts containing very high amounts of anthocyanins well in excess of what could be ingested as part of a normal berry-based diet.

As far as localization of other flavonoids within the body is concerned, acute supplementation of rats with $\left[2-{ }^{14} \mathrm{C}\right]$ quercetin$4^{\prime}$-O-glucoside at a dose of $4 \mathrm{mg} / \mathrm{kg}$ body weight is a study of relevance (323). The bulk of the ingested radiolabeled flavonol reached the cecum and colon, and it was rapidly degraded to phenolic acids, principally 3-hydroxyphenylacetic acid and benzoic acid. Most of radioactivity, over a 72-h period, was rapidly excreted in urine without any noticeable buildup in the circulatory system or body tissues, including the brain. In contrast, feeds of unlabeled quercetin to rats at substantially higher doses of 50 and $200 \mathrm{mg} / \mathrm{kg}$, corresponding to intakes of 271 and $1084 \mathrm{mg}$ for a $70-\mathrm{kg}$ human, did result in the detection of trace levels of quercetin in perfused rat brain tissues (216). In a human study in which volunteers drank $250 \mathrm{ml}$ of green tea containing $505 \mu \mathrm{mol}$ of flavan-3-ol monomers, typical (epi)catechin and (epi)gallocatechin metabolites were detected in plasma $1 \mathrm{~h}$ after ingestion, but they were not present in the cerebrospinal fluid collected $1 \mathrm{~h}$ later (513). In contrast, small amounts of radioactivity were detected in the brain of rats 6 and $24 \mathrm{~h}$ after feeding $\left[{ }^{3} \mathrm{H}\right]-(-)$-epigallocatechin-3-O-gallate by gavage (449). Radioactivity was also detected in the brain of rats after feeding mixtures of ${ }^{14} \mathrm{C}$-labeled grape (poly)phenols (222). However, in neither of these studies was the identity of radiolabeled compounds in brain tissues determined.

There is a report on the detection of flavan-3-ols in extracts of glucuronidase/sulfatase-treated prostate biopsy tissue obtained from subjects who had consumed $1.42 \mathrm{~L}$ of either green or black tea for a period of 5 days (192), and as noted earlier, after consumption of pomegranate juice and walnuts, urolithin A glucuronide and traces of urolithin B have also been detected in human prostate tissue (162). In a further study, glucuronide metabolites of epicatechin and methylepicatechin were detected in the thymus, testes, and liver, with lower amounts in the lymph nodes and spleen, of rats that had been fed a 10\% cocoa diet for a period of 15 days (466). In addition, after the ingestion of isoflavones, the presence of daidzein and genistein and their metabolites equol, enterolactone, and enterodiol has also been detected in human prostate tissue, whereas after ingesting $\sim 300 \mu \mathrm{mol}$ of daidzein, concentrations up to $6 \mu \mathrm{M}$ of equol accumulated in the breast tissue [see(93)].
Immunohistochemical studies have established that quercetin-3-O-glucuronide (Fig. 15), one of the main quercetin metabolites in the circulatory system (105), accumulates in macrophage-derived foam cells of human atherosclerotic lesions, but not in the normal aorta (243). In vitro experiments with murine macrophage cell lines showed that quercetin-3O-glucuronide was taken up and deconjugated to its more bioactive aglycone quercetin, which was in turn partially converted to a methylated metabolite. In addition, mRNA expression of the class A scavenger receptor and CD36, which play key roles in the formation of foam cells, was suppressed by treatment with quercetin-3-O-glucuronide (243). Likewise, immunohistochemical studies have also shown that (-)epicatechin-3-O-gallate, a constituent of green tea that appears in plasma in trace amounts after ingestion (438), is also specifically localized in macrophage-derived foam cells and similarly suppresses gene expression of CD36 (244).

\section{In Vitro Biological Activity and Mode of Action}

Bioactivity investigations using human or animal cell lines have made extensive use of both (poly)phenol aglycones and their sugar conjugates, the latter being the typical form in which they exist in planta. The concentrations at which these compounds have been assayed have usually been in the low$\mu M$-to-m $M$ range $(340,385,502)$. However, as outlined in previous sections of this review, after ingestion, with few exceptions, dietary (poly)phenolics appear in the circulatory system not as the parent compounds, but as glucuronide, methyl, and sulfate metabolites, and their presence in plasma after normal dietary intake rarely exceeds $\mathrm{n} M$ concentrations, even at $C_{\max }(93,109)$. Moreover, substantial quantities of (poly)phenols are not absorbed in the small intestine, but pass to the colon where they are degraded by the action of the microbiota, to give rise to a plethora of small phenolic acid and aromatic catabolites that are absorbed into the circulatory system before being excreted in quantities that vastly exceed those of metabolites absorbed in the small intestine (108). Therefore, the focus of this section of the review will be on the in vitro bioactivity of in vivo metabolites and catabolites formed within body after intake of dietary-relevant doses of (poly)phenol-rich foods.

\section{A. Anthocyanins}

Protocatechuic acid (3,4-dihydroxybenzoic acid) is one of the catabolites derived from colonic microbial degradation of cyanidin-O-glucosides $(159,478)$ (see Fig. 19), and its bioactivity in vitro has been investigated in some detail, with its influence in cell proliferation and death being among the areas of interest. It has apoptotic effects on human gastric adenocarcinoma cells in a time- and dose-dependent manner (280). This study also showed that the effect of protocatechuic acid on an adenocarcinoma cell line might be mediated via sustained phosphorylation and activation of c-Jun N-terminal kinase (JNK) and p38 mitogen-activated protein kinase (MAPK), but not extracellular-signal-regulated kinases (ERK), thus defining a very specific mechanism of action. This is in good agreement with Yip et al. (508), who reported cytotoxicity of protocatechuic acid against HepG2 hepatocellular carcinoma cells exerted by stimulating JNK and p38 subgroups of the MAPK family. In contrast to these 
proapoptotic effects, protocatechuic acid was able to increase the viability of cultured neural stem cells and to stimulate cell proliferation. The reduced level of apoptosis of neural stem cells was probably brought about by lowering the level of reactive oxygen species (ROS) and decreasing the activity of caspase-3 (172). This indicates that protocatechuic acid can act in varying ways in different environments, acting in a proapoptotic manner in actively proliferating cells, while protecting normal cells from apoptosis. This antiapoptotic ability was seemingly confirmed in a study in which treatment of cultured rat adrenal gland pheochromocytoma (PC12) cells with protocatechuic acid prevented 1-methyl-4phenylpyridinium ion $\left(\mathrm{MPP}^{+}\right)$-induced loss of mitochondrial membrane potential, formation of ROS, glutathione depletion, activation of caspase-3, and downregulation of Bcl-2 (173). However, the concentration of protocatechuic acid used to treat cells in these studies ranged from 0.06 to $8 \mathrm{mM}$, which vastly exceeds the levels likely to be attained in vivo (478), making the reported effects of this anthocyanin catabolite of questionable physiological relevance, in this instance.

Consumption of fruits and vegetables and related products, rich in anthocyanins and other (poly)phenols, is thought to reduce inflammatory stress, and as a consequence, provides some degree of protection against the development of chronic diseases, including cancer, cardiovascular, and neurodegenerative pathologies $(4,150,402)$. From the inflammation perspective, protocatechuic acid has been reported to inhibit monocyte adhesion to tumour necrosis factor- $\alpha$ (TNF$\alpha$ )-activated mouse aortic endothelial cells, and also to reduce vascular cell adhesion molecule 1 (VCAM-1), intercellular adhesion molecule 1 (ICAM-1) expression, the nuclear content of p65, and nuclear factor $\kappa \mathrm{B}$ (NF- $\kappa \mathrm{B})$-binding activity (481). In the same study, protocatechuic acid administered to apolipoprotein E (ApoE)-deficient mice reduced aortic VCAM-1 and ICAM-1 expression, NF- $\kappa$ B activity, and plasma-soluble VCAM-1 and ICAM-1 levels, thus inhibiting the development of atherosclerosis. These results suggest that protocatechuic acid could be considered as at least partially antiatherogenic because of its vascular anti-inflammatory activity.

Verzelloni et al. (476) observed that 3'-hydroxyphenylacetic acid, 3',4'-dihydroxyphenylacetic acid, and $3^{\prime}$-methoxy-4'hydroxyphenylacetic acid, known anthocyanin microbial catabolites (493), inhibited the formation of advanced glycation end products (AGEs) when applied as a mixture at a combined concentration of $2.0 \mu \mathrm{M}$. The same set of catabolites was effective in preserving cultivated neuron cells from death due to oxidative stress at a concentration of $1.5 \mu \mathrm{M}$. Additional experiments were carried out with the AGE model that pointed to the glucose-mediated pathway as the likely site of action of the catabolites. In another study by this group, hydroxycinnamate catabolites, derived from caffeoylquinic acids in coffee, were able to inhibit protein glycation, which was related to a reduction of Amadori products, suggesting that these compounds could act as pre-Amadori inhibitors of protein glycation (477). Since phenolic compounds have the ability to bind to proteins, it was hypothesized that the catabolites may protect albumin from glycation by a mechanism involving some form of specific chemical binding. These studies are good examples of testing the right compounds, as the phenolic catabolites known to be generated in vivo were able, at physiological concentrations, to counteract two key features of diabetic complications, at least in vitro, namely protein glycation and neurodegeneration.

Evidence exists for an inverse relationship between moderate intake of red wine and the incidence of rectal cancer (351), and this could be attributed to the colonic metabolites of malvidin-3-O-glucoside and other anthocyanins, which along with procyanidins are major components in many red wines. Forester and Waterhouse (144) tested the anthocyanin microbial catabolites gallic acid, 3-O-methyl-gallic acid, protocatechuic acid, vanillic acid (3-methoxy-4-hydroxybenzoic acid), syringic acid (3,5-dimethoxy-4-hydroxybenzoic acid), and 2,4,6-trihydroxybenzaldehyde for their ability to induce apoptosis and inhibit cell proliferation in a colon cancer model using Caco-2 cells. Gallic acid, 3-O-methyl-gallic acid, and 2,4,6-trihydroxybenzaldehyde all reduced cell proliferation without being cytotoxic and were more effective than the parent anthocyanins. The catabolites degraded rapidly in the culture medium, leaving open the possible involvement of subsequent breakdown products in the reduction in cell viability.

Russell et al. (396) compared the anti-inflammatory properties of blueberry microbial catabolites, mostly derivatives of benzoic acid, phenylacetic acid, and phenylpropionic acid, obtained by fermenting a blueberry extract with fecal slurries from two volunteers. This was done by assessing the ability of the catabolites to modulate prostanoid production in a cell system in which the inflammatory pathways were upregulated by application of a cytokineinduced stimulation. After interleukin-1 beta (IL-1 $\beta$ ) treatment of these cells, the catabolites generated by the fecal material from volunteer 1 decreased the amount of prostanoids produced, whereas the aromatic compounds produced by slurries from volunteer 2 had the opposite effect and increased prostanoid production. Although the number of volunteers used in this study makes any conclusions extremely tentative, the results suggest that the antiinflammatory effect of blueberry (poly)phenolics in the colon may be dependent upon microbial catabolism which, as noted reviously, can vary somewhat as a consequence of person-to-person diversity in the composition of the colonic microbiota.

\section{B. Chlorogenic acids}

Colonic metabolites of chlorogenic acids such as $m$ coumaric acid and dihydroferulic acid [3-(3'-methoxy-4'hydroxyphenyl)propionic acid] showed high antioxidant activity in a study carried out by Gómez-Ruiz et al. (155). However, despite these antioxidant properties, they were not able to protect human low-density lipoprotein (LDL) oxidation induced by copper and 2,2'-azobis(2-amidinopropane) dihydrochloride.

Platelet activation and subsequent aggregation play a major role in the pathogenesis of thromboembolic diseases such as myocardial infarction and ischemic heart disease. Hyperactive platelets with a high predisposition for activation are known to be apparent in conditions such as diabetes and heart disease (197). Dihydroferulic acid and 3-(3'hydroxyphenyl)propionic acid, chlorogenic acid, and anthocyanin colonic catabolites $(371,436)$ partly reversed hyper-reactivity of platelets induced by oxidative stress, and 
could also counteract the negative effects of hormonal stressinduced platelet hyper-reactivity (370).

The results regarding the other main chlorogenic acid catabolite, dihydrocaffeic acid [3-(3', 4'-dihydroxyphenyl) propionic acid], suggested that this compound is able to scavenge intracellular ROS (215). In the same study, dihydrocaffeic acid also increased nitric oxide (NO) synthase activity in a dose-dependent manner in cultured cells, with a comparable increase in endothelial $\mathrm{NO}$ synthase protein. Although the concentrations of the hydroxycinnamate required to achieve this effect were higher $(>50 \mu \mathrm{M})$ than those observed in plasma after ingestion of a cup of coffee (436), these results do indicate that dihydrocaffeic acid may function as an intracellular antioxidant.

The antioxidant character of dihydrocaffeic acid was confirmed in another study in which it reduced cytotoxicity and proinflammatory cytokine production (IL-6 and IL-8) in HaCaT cells, a keratinocyte model, after UV radiation. However, these effects may be attributed to direct radical scavenging of the ROS formed, and to reinforcement of the internal antioxidant defenses of the keratinocytes, as well as to a direct interference with the pathway involved in cytokine stimulation (358).

The fact that dihydrocaffeic acid is not just an antioxidant was convincingly established by Miene et al. (307), who demonstrated that it was able to upregulate glutathione $S$ transferase T2 (GSTT2) and decrease cyclooxygenase-2 (COX2) expression in human adenoma cells LT97 at concentrations ranging from 5 to $25 \mu \mathrm{M}$. Cumene hydroperoxide-induced DNA damage was significantly reduced by the catabolite. It was proposed that an upregulation of GSTT2 with a concomitant downregulation of COX-2 may contribute to the chemopreventive potential of (poly)phenols after degradation in the distal GIT.

Recently, chlorogenic acid-derived catabolites (dihydrocaffeic acid, dihydroferulic acid, and feruloylglycine) (436) (Fig. 34), when tested in combination at physiological concentrations, were shown to be effective in protecting human neuronal cells from oxidative insults in an in vitro experimental model (476). However, despite the fact that the induced cellular stress was of oxidative origin $(2 \mu M$ 2,3dimethoxy-1,4-naphthoquinone), the combination of chlorogenic acid-derived catabolites was the most effective, and independent of the antioxidant activity of the individual components, indicating that the protective mechanisms should not be attributed to the mere ability to prevent oxidation. Another ferulic acid catabolite, ferulaldehyde (aka coniferaldehyde) (Fig. 37), had been previously tested in a murine lipopolysaccharide (LPS)-induced septic shock model (367). It was found that intraperitoneally administered ferulaldehyde $(6 \mathrm{mg} / \mathrm{kg}$ every $12 \mathrm{~h})$ prolonged the lifespan of LPS-treated mice, decreased the inflammatory response, decreased early proinflammatory cytokines (TNF- $\alpha$ and IL- $1 \beta$ ), increased anti-inflammatory IL-10 levels in serum, and inhibited LPS-induced activation of NF- $\kappa$ B in the liver of the mice.

\section{Ellagitannins}

There is a rapidly growing body of literature describing the biological activities of ellagitannin-derived urolithins. The antioxidant activity of seven urolithins was evaluated in a cell-based assay (31), and the antioxidant activity was corre- lated with the number of hydroxyl groups and the lipophilicity of the urolithin molecule. The most potent antioxidants were urolithins $\mathrm{D}$ and $\mathrm{C}$, with four and three hydroxyl groups, respectively, whereas the dihydroxylated urolithin A exhibited only weak activity. However, all these urolithins possessed less antioxidant activity than their parent molecules, ellagic acid and punicalagins. Contrary to what was observed in this study, Ito (217) reported that urolithins exhibited more potent antioxidant activities in the oxygenradical absorbance capacity (ORAC) assay than intact ellagitannins, with urolithin A being among the most active.

However, as discussed with other components, antioxidant properties are not central and other more specific and biologically relevant activities are beginning to emerge. In a human colon cancer cell line, urolithin A and urolithin B, at concentrations achievable in the lumen from a dietary intake, influenced the expression levels of both signaling genes, such as growth factor receptors, oncogenes, and tumour suppressors, and genes involved in the cell cycle. These effects can be linked to cancer prevention in epithelial cells lining the colon (161). In a similar cell model, urolithins A and B induced the expression and activity of phase I and II enzymes, including cytochrome P450 mono-oxygenase 1A1 and UGT 1A10, and inhibited several SULTs (160). In a well-known rat model of inflammatory bowel disease, urolithins $\mathrm{A}$ and $\mathrm{B}$ reduced a number of inflammation markers (i.e., inducible NO synthase, COX-2, prostaglandin E [PGE] synthase, and PGE2 in colonic mucosa), favorably modulated the gut microbiota, preserved colonic architecture, but did not decrease oxidative stress in plasma and colon mucosa (271). Interestingly, in the same study, the formation of urolithins from punicalagin in rats fed a pomegranate extract was prevented by inflammation, suggesting that urolithin $\mathrm{A}$, in particular, could be an active antiinflammatory compound in healthy subjects, but that other molecules, arguably punicalagin, may be responsible for antiinflammatory activity when a pathological condition appears. In a subsequent study with colon fibroblasts (163), urolithin A and $\mathrm{B}(10 \mu \mathrm{M})$ inhibited prostaglandin E2 production after IL$1 \beta$ stimulation. Urolithin A, but not urolithin B, downregulated COX-2 and microsomal PGE synthase-1 (mPGES-1) mRNA expression and protein levels. Both urolithins inhibited NF- $\kappa$ B translocation to the nucleus. Slight, but significant, effects were found in the activation of MAPK pathways. Urolithin A lowered JNK phosphorylation state, and both urolithins inhibited p38 activation. Very interestingly, no urolithin-derived metabolites were found in the cell incubation medium, and only traces of urolithins were found inside the cells.

The current results, therefore, suggest that urolithins, the dihydroxy urolithin A in particular, have anti-inflammatory

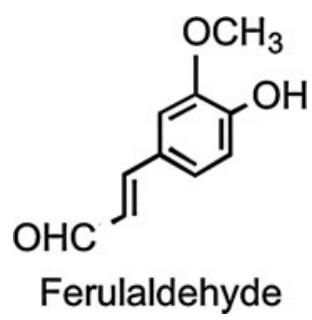

FIG. 37. Ferulaldehyde. 
Table 11. Mechanisms of Action Exerted by Ellagitannin Metabolites in Models Related to Cancer and Inflammation

\begin{tabular}{|c|c|c|c|}
\hline Metabolite & Mechanisms & Model/cell line & References \\
\hline Urolithin A & $\mathrm{PGE}_{2} \downarrow, \mathrm{COX}-2-, \mathrm{NF}-\kappa \mathrm{B} \downarrow, \mathrm{JNK} \downarrow$ & Colon fibroblast, & 163 \\
\hline Urolithin B & $\mathrm{PGE}_{2} \downarrow, \mathrm{COX}-2=, \mathrm{NF}-\kappa \mathrm{B} \downarrow, \mathrm{JNK}=$ & Colon fibroblast & 163 \\
\hline Ellagic acid & $\mathrm{PGE}_{2}=, \mathrm{COX}-2=, \mathrm{NF}-\kappa \mathrm{B}=, \mathrm{JNK}=$ & Colon fibroblast & 163 \\
\hline $\begin{array}{l}\text { Urolithins A and } \\
\mathrm{B}+\text { ellagic acid }\end{array}$ & $\begin{array}{l}\text { Cell cycle arrest, FGFR2-, } \\
\text { DUSP6 + , c-Myc +, }\end{array}$ & $\begin{array}{l}\text { Caco-2, microarray / } \\
\text { RT-PCR, WB }\end{array}$ & 160,161 \\
\hline Urolithin A & $\begin{array}{l}\text { Normal cell cycle, FGFR2-, } \\
\text { DUSP6 +, c-Myc +, CYP1A1 +, } \\
\text { UGT1A10+ }\end{array}$ & $\begin{array}{l}\text { Caco-2, microarray/ } \\
\text { RT-PCR; WB }\end{array}$ & 160,161 \\
\hline Urolithin B & $\begin{array}{l}\text { Normal cell cycle, FGFR2-, } \\
\text { DUSP6 + , c-Myc +, CYP1A1 +, } \\
\text { UGT1A10+ }\end{array}$ & $\begin{array}{l}\text { Caco-2, microarray/ } \\
\text { RT-PCR, WB }\end{array}$ & 160,161 \\
\hline Ellagic acid & $\begin{array}{l}\text { Normal cell cycle, FGFR2-, } \\
\text { DUSP6 +, c-Myc +, CYP1A1 +, } \\
\text { UGT1A10+ }\end{array}$ & $\begin{array}{l}\text { Caco-2, microarray/ } \\
\text { RT-PCR, WB }\end{array}$ & 160,161 \\
\hline Urolithin A & COX-2-, iNOS,- PTGES,$- \mathrm{PGE}_{2} \downarrow$ & $\begin{array}{l}\text { Rat inflammatory } \\
\text { bowel disease, WB }\end{array}$ & 271 \\
\hline
\end{tabular}

$\downarrow$, signaling/effect inhibition; =, no significant change; + , expression induction (upregulation), - , expression inhibition (downregulation); c-Myc, v-myc myelocytomatosis viral oncogene homolog (avian); COX-2, cyclooxygenase-2; CYP1B1, cytochrome p450 1B1; DUSP-6, dualspecificity phosphatase 6; FGFR2, fibroblast growth factor receptor 2; iNOS, inducible nitric oxide synthases; JNK, c-Jun N-terminal kinase; PTGES, prostaglandin E synthase; Uro A/B, urolithin A/B; VEGF, vascular endothelial growth factor; WB, western blot.

properties, and that the mode of action involves inhibition of NF- $\kappa$ B and MAPK activation, downregulation of COX-2 and mPGES-1 expression, and consequently, a reduction of PGE2 production. It must be pointed out that as urolithins were not able to efficiently enter the cells, interference for IL-1 $\beta$ membrane receptors cannot be excluded.

This anti-inflammatory ability of ellagitannin metabolites could be translated into a relevant cancer preventive effect, at least in the environment of the large intestine, where the concentration of these compounds is comparable to those applied in experimental in vitro models. To corroborate this hypothesis, in vitro urolithins were shown to inhibit 2,3,7,8tetrachlorodibenzo- $p$-dioxin-induced CYP1-mediated ethoxyresorufin- $O$-de-ethylase activity with $\mathrm{IC}_{50}$ values ranging from $57 \mu \mathrm{M}$ for urolithin A to $75 \mu \mathrm{M}$ for urolithin $\mathrm{C}$, which may be reasonably achievable concentrations in physiological conditions in the colon. These compounds, applied at similar concentrations, exhibited dose- and time-dependent effects in decreasing cell proliferation and the clonogenic efficiency of HT-29 cells. Inhibition of cell proliferation was mediated through cell cycle arrest at the $S$ phase, followed by the induction of apoptosis (241). These findings indicate that the urolithins released in the colon after consumption of ellagitannin-rich foods, such as walnuts, raspberries, or pomegranate juice (269), can potentially curtail the risk of colon cancer development by inhibiting cell proliferation and inducing apoptosis.

Another study investigated the effects of urolithins on Wnt signaling in a human $293 \mathrm{~T}$ cell line using a luciferase reporter of canonical Wnt pathway-mediated transcriptional activation (414). Investigating this pathway is extremely relevant as Wnt proteins are a family of highly conserved, secreted signaling molecules playing a pivotal role in cellular development and carcinogenesis, with a large percentage ( $\sim 90 \%)$ of colon cancers arising from activating mutations in the Wnt pathway (250). Urolithin A was able to inhibit Wnt signaling at physiological intestinal concentrations, suggesting that el- lagitannin-rich foods have the potential to prevent or counteract colon carcinogenesis through this novel mechanism.

Urolithin activity has also been investigated in the context of other forms of cancer. Seeram et al. (406) noted that urolithins accumulated in the prostate tissue of C57BL/ 6 wildtype male mice fed a pomegranate extract and demonstrated that urolithins were able to inhibit the growth of human prostate cancer cells in vitro. Subsequently, Kasimsetty et al. (241) reported that urolithin C, 8-O-methylurolithin A, and 8,9-di-O-methylurolithin $\mathrm{C}$ caused potent inhibition of CYP1-mediated ethoxyresorufin-O-de-ethylase activity also in 22Rv1 prostate carcinoma cells after a 24-h incubation at micromolar concentrations. Neutral red uptake assay results indicated that the same three metabolites induced profound cytotoxicity in the proximity of their CYP1 inhibitory $\mathrm{IC}_{50}$ values. Moreover, urolithins interfered with the expression of CYP1B1 protein, an established target in prostate cancer chemoprevention. Thus, urolithins were found to display a dual mode mechanism of cancer chemopreventive activity, by decreasing CYP1B1 activity and expression.

Urolithins A and B together with their acetylated, methylated, and sulfated analogs were examined for their ability to inhibit aromatase activity and testosterone-induced breast cancer cell proliferation at concentrations in the low micromolar range (1). Urolithin B was the most effective inhibitor of aromatase activity in a live cell assay. Kinetic analysis of urolithin B showed mixed inhibition, suggesting more than one inhibitory mechanism. Urolithin B was also the most effective in inhibiting testosterone-induced MCF-7aro cell proliferation, whereas the other urolithins were effective to a much lesser degree. These observations suggest that ellagitanninderived urolithin B has potential for the prevention of estrogen-responsive breast cancers. In fact, both urolithins A and B had been previously shown to exert estrogenic activity in a dose-dependent manner, even at high concentrations, and without antiproliferative or toxic effects in estrogen-sensitive human breast cancer MCF-7 cells, whereas common 
FIG. 38. The Eph-ephrin system regulates cell adhesion, shape and movements, cell proliferation, survival, differentiation, and secretion and plays a role in embryogenesis, neural development, plasticity and regeneration, angiogenesis, bone remodeling, insulin secretion, intestinal homeostasis, cancer suppression, and/or promotion (347). Pyrogallol (1,2,3-trihydroxybenzene), a common colonic metabolite of several (poly)phenolic compounds, has been reported as able to perturb the proteinprotein interactions in a dose-dependent way (457). (To see this illustration in color, the reader is referred to the web version of this article at www.liebertpub.com/ars.)

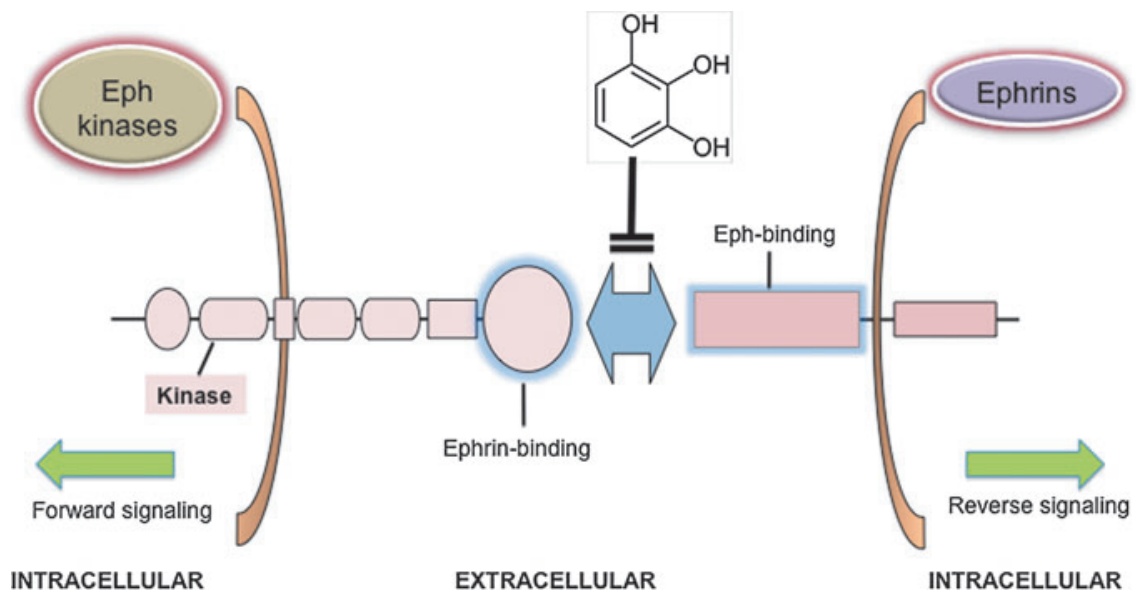

phytoestrogens such as isoflavones are reported to inhibit cell proliferation only at high concentrations (270). Overall, urolithins exhibited weaker estrogenic activity than other well-known phytoestrogens, but displayed a slightly higher antiestrogenic activity.

In the previously cited antiglycative experimental model, urolithins A and B, together with pyrogallol (1,2,3trihydroxybenzene), inhibited protein glycation by $37 \%$ and $44 \%$ at 1 and $2 \mu \mathrm{M}$, respectively, showing a potential effect in protecting circulating proteins during hyperglycemic conditions (476). Table 11 summarizes the mechanisms of action exerted by ellagitannin metabolites in models related to cancer and inflammation.

The Eph-ephrin system coordinates the physiology and homeostasis of many adult organs, and therefore, an imbalance of Eph-ephrin function may contribute to a variety of diseases, including neurodegeneration, diabetes, CVDs, and cancer (347). It has recently been demonstrated that pyrogallol is able to functionally interfere with the EphA2-ephrinA1 system. The concentrations used were relatively high, but considering the very high levels that could be reached in the colon after consumption of a wide array of different (poly)phenol-containing foods, interactions with colonic Ephephrin protein in vivo are feasible (457) (Fig. 38).

\section{Flavan-3-ols}

Once again, antioxidant activity of the main metabolites of flavan-3-ols has been tested in several studies. Starting with the phase II human metabolites, the $3^{\prime}-O$ - and 4'-O-methyl derivatives of $(+)$-catechin and $(-)$-epicatechin, have been characterized, and their antioxidant activity was evaluated and compared to those of the corresponding quercetin metabolites. The antioxidant activity was assessed using the ferric-reducing antioxidant power (FRAP) assay and two methods based on the ability to scavenge the $\mathrm{ABTS}^{\bullet+}$ radical cation at different $\mathrm{pH}$ values (121). The $\mathrm{O}$-methylation at the B-ring level resulted in reduced antioxidant activity with respect to the parent compounds. However, the methylated metabolites still retained significant radical-scavenging activity at $\mathrm{pH} 7.4$, suggesting that they could act as potential antioxidants under physiological conditions. The highest antioxidant activity was that of $3^{\prime}$-O-methyl-catechin at $\mathrm{pH}$
7.4, comparable to those of its parent compound and the quercetin metabolites. In a more recent study, (-)-epigallocatechin-3-O-gallate exhibited antioxidant activity more than five times higher than that of Trolox, whereas that of $4^{\prime}, 4^{\prime \prime}$ di-O-methyl-epigallocatechin-3-O-gallate was similar to Trolox. This observation demonstrates that dimethylation of (-)-epigallocatechin-3-O-gallate results in a dramatic loss of its reducing activity (285). Applying the 2,2-diphenyl-1-picrylhydrazyl (DPPH) antioxidant assay, it has also been reported that epigallocatechin-3-O-gallate-3'-O-glucuronide and epigallocatechin3-O-gallate-3-O-glucuronide have an antioxidant capacity similar to that of (-)-epigallocatechin-3-O-gallate. Epigallocatechin3-O-gallate-4"-O-glucuronide, epigallocatechin-3-O-gallate-7O-glucuronide, and epigallocatechin-7-O-glucuronide were less active than their parent compounds, and epigallocatechin-3'-Oglucuronide was much less active than (-)-epigallocatechin (286).

Regarding flavan-3-ol colon-derived catabolites, the antioxidant activity of 5-( $3^{\prime}, 4^{\prime}$-dihydroxyphenyl)- $\gamma$-valerolactone (M6), 5-(3',5'-dihydroxyphenyl)- $\gamma$-valerolactone (M6'), 5 -( $3^{\prime}, 4^{\prime}, 5^{\prime}$-trihydroxyphenyl)- $\gamma$-valerolactone (M4), and 5(3'-hydroxyphenyl)- $\gamma$-valerolactone (M7) was calculated by Takagaki et al. (452). The order of their antioxidant activity in this study was M4 > M6' > M6 > M7.

As already discussed, the antioxidant activity of (poly)phenol metabolites, even when they retain the activity of their unmetabolized parent compounds, is not necessarily the most relevant feature of their putative biological activity. Koga and Meydani (251) obtained plasma extracts of flavonoid metabolites prepared after intragastric administration of pure compounds to rats. The extracts contained a mixture of sulfate, glucuronide, and methyl metabolites. The investigators measured the adhesion of U937 monocytic cells to human aortic endothelial cells and the production of ROS in these cells when they were pretreated with either pure compounds or plasma extracts from control or treated rats. The data obtained demonstrated that pretreatment of endothelial cells with methylated (+)-catechin metabolites inhibited U937 cell adhesion, whereas (+)-catechin had no effect. Generation of ROS in hydrogen peroxide-stimulated endothelium was inhibited by (+)-catechin, methyl-catechin, and,unexpectedly, the control plasma extract, whereas ROS generation in IL- $1 \beta$ stimulated endothelial cells was inhibited only by methylcatechin. The merit of this 2001 report is that it was one of the 
first to point out that the metabolites of flavonoids, rather than their parent compounds, may contribute to the epidemiologically reported effects of flavonoids in terms of reducing the risk of CVD. However, the approach used and the tested metabolites are far from mimicking human physiology, as methylcatechin is not a common circulating metabolite in humans, and applying plasma extracts to cell cultures does not facilitate distinguishing between the molecules that exert a biological effect.

Methylated flavan-3-ols were reinvestigated in a more recent study that addressed the effects of (-)-epicatechin and some of its phase II human metabolites on NADPH oxidase activity in intact human umbilical vein endothelial cells (HUVECs) and in cell lysates (442). The parent flavan-3-ol proved to be an $\mathrm{O}_{2}{ }^{\bullet+}$-scavenger, but did not inhibit NADPH oxidase activity, whereas the opposite was observed for $3^{\prime}$ and 4'-O-methyl-(-)-epicatechin. In contrast, the dimer procyanidin B2 and an epicatechin-O-glucuronide, in which the position of the glucuronide moiety was not stated, were both $\mathrm{O}_{2}^{\bullet+}$-scavengers and inhibited NADPH oxidase.

Lu et al. (286) reported that epicatechin metabolites isolated from human and rat urine inhibited peroxynitrite-mediated tyrosine nitration, with the following order of potency: (-)-epicatechin-3'-O-glucuronide $>(-)$-epicatechin $>(-)$ epicatechin-7-O-glucuronide $=3^{\prime}-O-$ methyl- $(-)$-epicatechin-7$O$-glucuronide $=4^{\prime}-O$-methyl- $(-)$-epicatechin-3'-O-glucuronide. These results demonstrate that the metabolites of (-)-epicatechin can prevent peroxynitrite-induced physiological damages, to some extent, as oxidation of tyrosine residues, which is associated with the nitration of proteins, is of particular interest in pathologies related to inflammation. The relevance of these metabolites in the context of inflammation has been confirmed in other studies. At concentrations of 2 and $10 \mu \mathrm{M}$, which can be considered physiologically relevant, several flavan-3-ol glucuronides inhibited the release of arachidonic acid and its metabolites from HT-29 cells (286). At both concentrations, epigallocatechin-3'-O-glucuronide was less effective than epigallocatechin-7-O-glucuronide, which had comparable activity to (-)-epigallocatechin. Ascorbic acid had no effect on arachidonic acid release at concentrations as high as $50 \mu \mathrm{M}$, suggesting a mechanism that does not involve antioxidant activity. In a more recent study (285), 4',4"-di-O-methyl-epigallocatechin-3$O$-gallate selectively inhibited ICAM-1 expression with an $\mathrm{IC}_{50}$ value of $94 \mu \mathrm{M}$, whereas (-)-epigallocatechin-3-O-gallate and 4"-O-methyl-epigallocatechin-3-O-gallate had no effect. The inhibitory effect of $4^{\prime}, 4^{\prime \prime}$-di-O-methyl-epigallocatechin-3-O-gallate on adhesion molecule expression was not related to either its antioxidant-reducing capacity or an inhibition of $\mathrm{NF}-\kappa \mathrm{B}$ activation.

A few studies have investigated the biological activity of the microbial flavan-3-ol catabolites, the valerolactones. Two, M6 and its methylated analog 5-(3'-methoxy-4'-hydroxyphenyl)- $\gamma$-valerolactone, are catechin catabolites identified in human urine after the consumption of pine bark extract. These two molecules exhibited strong inhibitory activity toward metalloproteinases (MMP) 1, 2, and 9. Moreover, at the cellular level, $0.5 \mu \mathrm{M}$ concentrations of both valerolactones resulted in a $\sim 50 \%$ inhibition of MMP-9 secretion. M6 was more effective in superoxide scavenging than (+)-catechin, ascorbic acid, and Trolox, whereas its 3'-methylated analog lacked scavenging activity (170). In another study, M4, M6, and their methoxy-derivatives were assessed for their ability to inhibit the growth of a panel of immortalized and malignant human cell lines using the 3-(4,5-dimethylthiazol-2-yl)-2,5diphenyltetrazolium bromide (MTT) assay. M4 was the most active catabolite tested on human esophageal squamous cell carcinoma cells (KYSE150), human colon adenocarcinoma cells (HT-29 and HCT-116), immortalized human intestinal epithelial cells (INT-407), and an immortalized rat intestinal epithelial cell line (IEC-6) (266). This valerolactone was also able to inhibit the production of NO by LPS-stimulated murine macrophages (RAW264.7). The growth inhibitory activity of M4 against immortalized and cancer cell lines allows us to hypothesize that this catabolite may contribute to the cancer preventive effect of green tea observed in epidemiological studies. The peculiar sensitivity of INT-407 cells relative to the other cell lines may indicate that the trihydroxyvalerolactone has some selectivity for premalignant cells, but this remains to be more thoroughly investigated. Moreover, the ability of M4 to inhibit the production of LPS-stimulated macrophages may indicate that it can contribute to the anti-inflammatory activities of green tea.

Although the valerolactone metabolites of flavan-3-ols have been found in human internal compartments at levels that vastly exceed those of many other flavonoid metabolites (277), there are relative few reports on their biological activities in in vitro test systems. This is because standards cannot be obtained from commercial sources. However, chemical procedures for their synthesis, and that of their sulfated and glucuronide metabolites, are available (25), so information on their mode of action is likely to be forthcoming in the near future.

\section{E. Flavonols}

Flavonols, quercetin in particular, are a notable exception to what has been written in this review, as studies dealing with the bioactivity of their human metabolites date back more than 10 years ago.

Quercetin metabolites retain a part of the antioxidant activity of their parent aglycone, and this has been demonstrated in several different experimental models. Indirectly, plasma obtained from rats fed quercetin was more resistant to copper sulfate-induced lipid peroxidation than the control plasma on the basis of the accumulation of hydroperoxides and the degradation of $\alpha$-tocopherol. Based on the fact that quercetin is recovered in rat blood plasma principally as sulfate and glucuronide conjugates, these observations indicate that at least some metabolites of quercetin retain the ability to act as effective antioxidants (98). To clarify the antioxidant properties of one of the main phase II metabolites of quercetin in a cellular environment, the ability of quercetin-3-O-glucuronide (Fig. 15) to inhibit the production of $\mathrm{H}_{2} \mathrm{O}_{2}$-induced ROS in mouse 3T3-cultured fibroblasts was evaluated (418). When the cells were exposed to $\mathrm{H}_{2} \mathrm{O}_{2}$ in the presence of quercetin or its glucuronide, the metabolite was less active than the aglycone. In contrast, when fibroblasts were pretreated with the flavonols before exposure to $\mathrm{H}_{2} \mathrm{O}_{2}$, the glucuronide, but not the aglycone, inhibited the production of ROS. In a different model of oxidation, both 3'-methylation and 3-glucuronidation significantly reduced the ability of quercetin to protect against radical-induced $\left[2,2^{\prime}\right.$-azobis(2methylpropionamidine)dihydrochloride] oxidation (283). In contrast, however, methylated quercetin metabolites (namely 3'-O- and 4'-O-methyl-quercetin) retained some antioxidant activity when tested in the FRAP and the 2,2'-azino-bis 
(3-ethylbenzothiazoline-6-sulfonic acid) (ABTS) assays (121). In another study, 3'-O-methylquercetin and quercetin-3'-Osulfate were shown to have lower reducing activity than quercetin in the FRAP assay (285).

The inhibitory effect of quercetin-3-O-glucuronide on lipid peroxidation has been investigated using phosphatidylcholine large unilamellar vesicles as a plasma membrane model. The glucuronide inhibited lipid peroxidation generated via different initiators, but less effectively than quercetin. Ultrafiltration of phosphatidylcholine large unilamellar vesicles revealed that the metabolite exhibited a low, but significant, affinity for the membranes of phospholipid bilayers, and therefore may be able to act as an efficient antioxidant in cell membranes (417).

Quercetin colonic catabolites have also been evaluated for their antioxidant activity. When measured with the DPPH assay, $3^{\prime}, 4^{\prime}$-dihydroxyphenylacetic acid exerted a concentration-dependent scavenging effect in the range of 1-30 $\mu \mathrm{M}$, whereas 4'-hydroxyphenylacetic acid was almost ineffective up to $500 \mu M$ (153). It has also been shown that $3^{\prime}, 4^{\prime}$-dihydroxyphenylacetic acid, 3,4-dihydroxybenzoic acid, and taxifolin exhibit antioxidant activity in the FRAP assay, but no reaction was observed with 3'-hydroxyphenylacetic acid, 4hydroxybenzoic acid, and 3-(3'-hydroxyphenyl)propionic acid (221). In part, this contrasts with the findings of Pavlica and Gebhardt (349), who reported that the lipophilic metabolite 3,4-dihydroxytoluene exhibited strong antilipoperoxidation activity, whereas the hydrophilic metabolite 3', 4'-dihydroxyphenylacetic acid was less effective against oxidative stress induced in PC12 cells by peroxides and iron.

In a pioneering study, Day et al. (103) reported on the antiinflammatory activity, measured as the ability to inhibit xanthine oxidase and lipoxygenase, of several quercetin glucuronides. The $\mathrm{K}_{\mathrm{i}}$ for the inhibition of xanthine oxidase by the glucuronide metabolites was as follows: $4^{\prime}>3^{\prime}>7>3$, with quercetin-4'-O-glucuronide being a particularly potent inhibitor $\left(K_{\mathrm{i}}=0.25 \mu M\right)$.

The anti-inflammatory activity of quercetin metabolites has subsequently been investigated by other groups with a particular attention being paid to the vascular system as a promising target of these molecules. It has, for instance, been shown that 3'-O-methyl-quercetin and 4'-O-methyl-quercetin inhibit the expression of ICAM- 1 at physiological concentrations in human aortic endothelial cells. E-selectin expression was also suppressed by both metabolites to a somewhat lesser extent, whereas VCAM-1 was unaffected. In contrast, quercetin-3-O-glucuronide, quercetin-3'-O-sulfate, and phenolic acid catabolites of quercetin were unable to inhibit adhesion molecule expression (285). This is in partial agreement with a previous study in which quercetin-3'-O-sulfate, quercetin-3$\mathrm{O}$-glucuronide, and isorhamnetin 3-O-glucuronide either exhibited a reduced ability to inhibit the expression of these molecules compared with the parent aglycone or had no effect. However, all three metabolites inhibited VCAM-1 cell surface expression at a concentration of $2 \mu M$ (459). These results, taken together, indicate that quercetin metabolites, at physiological concentrations, can inhibit the expression of key molecules involved in monocyte recruitment during the early stages of atherosclerosis. However, in a study on human umbilical artery smooth muscle cells activated by TNF- $\alpha$, these observations were not confirmed; quercetin- $3^{\prime}-O$-sulfate, quercetin-3-O-glucuronide, and isorhamnetin-3-O-glu- curonide had no effect on either TNF $\alpha$-induced upregulation of adhesion molecules (VCAM-1 and ICAM-1) or chemokine expression (monocyte chemotactic protein-1 [MCP-1]) (496).

These studies suggest that the vascular anti-inflammatory effects of quercetin consumption are mediated through effects on endothelial cells, but not smooth muscle cells. Moreover, quercetin-3'-O-sulfate and quercetin-3-O-glucuronide exert anti-inflammatory effects on the vasculature, possibly through a mechanism involving inhibition of $\mathrm{NF} \kappa \mathrm{B}$, by preventing LPS-induced changes in vascular responses in porcine coronary artery segments at physiological concentrations (5). It has also been reported that quercetin and isorhamnetin, but not quercetin-3-O-glucuronide, decrease mRNA and protein levels of TNF- $\alpha$ in murine RAW264.7 macrophages stimulated with LPS. Furthermore, no significant decreases in the mRNA levels of IL- $1 \beta$, IL- 6 , macrophage inflammatory protein- $1 \alpha$, and inducible NO synthase or proinflammatory microRNA155 were evident in response to quercetin glucuronide treatment (36). It can be concluded that the anti-inflammatory effectiveness of quercetin phase II metabolites is probably strongly linked to specific tissues and specific conditions, and that further research is needed to clarify these inconsistencies.

Modulation of endothelial function by quercetin metabolites, which has a strong connection with inflammation, has also been investigated. In a study involving thoracic aortic rings isolated from Wistar rats, quercetin-3-O-glucuronide, isorhamnetin-3-O-glucuronide, and quercetin-3'-O-sulfate had no direct vasorelaxant effects, nor did they modify endothelial function or the biological activity of NO. However, all metabolites, at marginally supraphysiological concentrations, partially prevented the impairment of the endothelialderived NO response under conditions of high oxidative stress induced by diethyldithiocarbamic acid, a superoxide dismutase inhibitor, and protected the biological activity of exogenous NO. Quercetin-3'-O-sulfate and quercetin-3-Oglucuronide inhibited NADPH oxidase-derived $\mathrm{O}_{2}$ release, but this occurred only at very high concentrations. Quercetin3-O-glucuronide was able to prevent the endothelial dysfunction induced by incubation with endothelin-1 (282). Subsequently, quercetin phase II conjugates were tested on vasoconstrictor and vasodilator responses in the porcine isolated coronary artery (451). Quercetin-3'-O-sulfate inhibited receptor-mediated contractions of the porcine isolated coronary artery by an endothelium-independent action and was able to counteract glyceryl trinitrate-induced tolerance in vitro, which may be beneficial for patients treated for angina pectoris.

Another study investigated the effect of quercetin sulfate and glucuronide metabolites on the prevention of high-glucose-induced apoptosis of HUVECs. HUVECs were exposed to high-glucose concentrations $(33 \mathrm{mM})$ in the presence or absence of physiological concentrations of a mixture of sulfated and glucuronidated quercetin isolated from rat plasma. High-glucose-induced apoptosis was inhibited by both metabolites at $\mathrm{nM}$ concentrations in a dose-dependent manner, through a mechanism involving $\mathrm{H}_{2} \mathrm{O}_{2}$ quenching and the inhibition of JNK and caspase-3 activity (71).

Donnini et al. (117) observed opposing effects of quercetin metabolites on angiogenesis. While quercetin-3-O-glucuronide inhibited vascular endothelial growth factor (VEGF)-induced endothelial cell functions and angiogenesis, quercetin-3'-Osulfate promoted endothelial cell proliferation and 
angiogenesis, and both were tested in the $\mathrm{n} M$ range. The inhibitory effect elicited by the glucuronide was associated with the inhibition of ERK1/2 phosphorylation elicited by VEGF. On the other hand, the activation of endothelial cells by the sulfate was associated with stimulation of VEGF receptor-2 and with downstream signaling activation (phosphatidylinositol-3 kinase/Akt and NO synthase pathways), ultimately responsible for ERK1/2 phosphorylation. This observation indicates how quercetin metabolism in vivo can drastically change its bioactivity, shifting it from one effect to the other.

The effects of quercetin phase II metabolites on lung cancer cells have also been investigated. Yang et al. (506) demonstrated that quercetin glucuronides (not fully identified, as they were prepared from the serum of quercetin-fed rabbits) were able to inhibit proliferation through G2/M arrest of the cell cycle and induce apoptosis via the caspase- 3 cascade in the human lung cancer cell line NCI-H209. These results were partly confirmed by Yeh et al. (507), who investigated the effects of quercetin metabolite-enriched plasma (QMP, obtained from Mongolian gerbils $2 \mathrm{~h}$ after quercetin feeding) on the growth of A549 lung cancer cells and the possible mechanisms of these effects. QMP, but not control plasma, reduced cell growth and led to cell cycle arrest at the G2/M phase by downregulating the expression of cdk1 and cyclin B. QMP, but not control plasma or quercetin aglycone, significantly increased peroxisome proliferator-activated receptor gamma (PPAR- $\gamma$ ) expression, which was accompanied by an increase in phosphatase and tensin homolog deleted on chromosome 10 and a decrease in the phosphorylation of Akt. Moreover, quercetin-3-O-glucuronide and quercetin-3'-O-sulfate also significantly increased PPAR- $\gamma$ expression in these cultured lung cancer cells. Finally, GW9662, a PPAR- $\gamma$ antagonist, significantly suppressed the effects of QMP on cell proliferation and on the expression of cyclin B and cdk1. Taken together, these data suggest that the interaction with PPAR- $\gamma$ could play a paramount role in the antiproliferative effects of quercetin metabolites.

The hypothesis of Terao and colleagues that glucuronide conjugates of quercetin are bioactive agents against ROS, but only as precursors of hydrophobic aglycones $(216,243)$, merits comment. Quercetin aglycone was predicted to be generated at the target site (i.e., the cardiovascular system or central nervous system) by the action of $\beta$-glucuronidase activity, under oxidative stress or inflammatory conditions, on quercetin-O-glucuronide metabolites. This hypothesis goes some way to restoring the reputation of at least some of the numerous studies performed with aglycones such as quercetin. However, as yet, the generation of quercetin has not been firmly established, and the presence of its glucuronide metabolites at the extracellular level would still require that all in vitro tests be performed with these molecules.

One of the main colonic catabolites of quercetin, $3^{\prime}, 4^{\prime}$-dihydroxyphenylacetic acid (Fig. 16), has been found to possess significant reducing power and free-radical scavenging activity (221). However, the same molecule has only limited activity in the prevention of cell death, glutathione depletion, lipid peroxidation, and production of ROS in differentiated PC12 cells, used as a model system of neuronal cells (349). This finding was confirmed by Verzelloni et al. (476), who observed a negligible effect of $3^{\prime}, 4^{\prime}$-dihydroxyphenylacetic acid, at physiological concentrations, on neuroblastoma cell protection against oxidative stress. This study also tested antiglycative activity and found that $3^{\prime}, 4^{\prime}$-dihydroxyphenylacetic acid was almost ineffective in preventing serum albumin glycation. However, this observation is in contrast to the results of Pashikanti et al. (346), who found that the phenolic acid catabolite was a powerful inhibitor of $\mathrm{N}^{\varepsilon}$-carboxymethyl lysine and $\mathrm{N}^{\varepsilon}$-carboxymethyl lysinehistone $\mathrm{H} 1$ adduct formation and ADP-ribose histone $\mathrm{H} 1$ glycation. Finally, Miene et al. (307) demonstrated that $3^{\prime}, 4^{\prime}-$ dihydroxyphenylacetic acid was, like 3-( $3^{\prime}, 4^{\prime}$-dihydroxyphenyl)propionic acid (dihydrocaffeic acid), able to act as an anti-inflammatory agent, possibly contributing to the chemopreventive potential of (poly)phenol catabolites in the gut.

\section{F. Lignans and isoflavones}

Phytoestrogens, plant-derived compounds structurally similar to estrogen, may have both agonistic and antagonistic actions toward estrogen receptors (460). There are two major classes of phytoestrogens: lignans, which occur in high amounts in flaxseeds (Fig. 5), and isoflavones, found in high concentrations in soy with the 7-O-glucoside and 7-O-(6"-Omalonyl)glucoside of genistein and daidzein being the main components (Fig. 4) (see Section II.A.3).

After ingestion, plant lignans are converted by the intestinal microbiota in the upper part of the large bowel to enterolactone and enterodiol (Fig. 36), which are referred to as mammalian enterolignans (2). Chen et al. (73) reported that enterolactone, at $\mu M$ concentrations, suppressed the growth of LNCaP prostate cancer cells by triggering apoptosis. The mechanism of action involved a dose-dependent loss of mitochondrial membrane potential, the release of cytochrome C, and cleavage of procaspase- 3 and poly(ADP-ribose) polymerase. A series of other intracellular proteins (Akt, GSK-3B, MDM2, and p53) were shown to be involved in enterolactonedependent apoptosis. To better understand how these estrogenic metabolites might influence breast cancer progression and to unravel their mechanisms of action, Carreau et al. (64) compared the ability of enterodiol and enterolactone to induce the transactivation of the two estrogen receptors (ERs), $\alpha$ and $\beta$, to modulate $\mathrm{ER} \alpha$ target genes and MCF-7 breast cancer cell migration by acting on matrix MMPs at a concentration of $10 \mu M$, which arguably is unlikely to be achieved via a lignanrich diet. It was found that enterolignans have distinct abilities for the activation of $\operatorname{ER} \alpha$ and $\operatorname{ER} \beta$ and modulation of $E R \alpha$ mRNA and protein contents and cell proliferation. The immunomodulatory activity of enterolactone and enterodiol was also investigated by treating peripheral blood lymphocytes with increasing physiologically relevant concentrations of the two lignan metabolites after stimulus with LPS and anti-CD3 and anti-CD28 monoclonal antibodies (87). A doserelated inhibition of cell proliferation and cytokine production was observed, with enterolactone being the most effective metabolite. Additional experiments with THP-1 cells showed that both compounds prevented inhibitory- $\kappa \mathrm{B}$ degradation and NF $-\kappa \mathrm{B}$ activation, resulting in decreased TNF- $\alpha$ production.

After ingestion by humans, genistein and daidzein glycosides undergo a rapid degradation by the gut microbiota, giving rise to a wide range of metabolites, including dihydrodaidzein, dihydrogenistein, equol, and O-desmethylangolensin (15) (see Section II.D). Among these metabolites, equol has been investigated most extensively. It was reported 
to generate acute endothelium- and NO-dependent relaxation of aortic rings, a rapid, 2-min activation of endothelial nitric oxide synthase (eNOS) in human aortic and umbilical vein endothelial cells at physiological concentrations (1-100 $\mathrm{nM}$ ) (232). The effects of equol on markers of immune function have been tested in human peripheral blood mononuclear cells. The evaluated variables included lytic activity of natural killer cells, proliferation, secretion of cytokines, and apoptosis/necrosis. In addition, the cells were treated with estrogenic antagonists to determine whether an estrogen receptor-mediated pathway was involved (169). It was shown that equol was a potent modulator of immune function, but the effect was evident only at high, nonphysiological concentrations $(10-50 \mu \mathrm{M})$. Particularly noteworthy was the observation that the effects on cytokine secretion were not mediated by phytoestrogenic activity.

Li et al. (279) investigated the ability of equol to activate human and mouse pregnane $X$ receptor (PXR), an inducer of drug metabolism. In human primary hepatocytes, equol was a potent activator of PXR, with consequent increase of CYP3A4 mRNA and immunoreactive protein expression, whereas genistein or daidzein was more effective on mouse PXR. Interestingly, these observations are consistent with speciesspecific differences in cytochrome P450-mediated drug and steroid metabolism.

Equol was also able to prevent oxidized LDL-stimulated apoptosis in HUVECs at a concentration of $1 \mu \mathrm{M}$, but was most effective at $10 \mu \mathrm{M}$. The mechanism of action was described as being related mainly to the control of superoxide and NO intracellular production (238). Endothelial function was also investigated by Rowlands et al. (394), who reported that equol-stimulated mitochondrial ROS modulate endothelial redox signaling and NO release, involving transactivation of epidermal growth factor receptor kinase and reorganization of the F-actin cytoskeleton, providing valuable insights for therapeutic strategies to restore endothelial function in CVD.

In another set of endothelial cells, human pulmonary artery endothelial cells, treatment with the HIV protease inhibitor ritonavir significantly reduced eNOS expression, and $1 \mu \mathrm{M}$ equol effectively blocked this downregulation (74). In the same study, ritonavir significantly reduced endothelium-dependent relaxation in response to bradykinin in fresh porcine pulmonary artery rings, and equol was able to reverse this effect. The results of this study suggest that equol may have a clinical application in providing some prevention against HIV treatment-associated cardiovascular complications.

\section{G. Flavanones}

The interactions of a mixture of hesperetin 5-O- and 7-Oglucuronides with human fibroblasts and their implications for oxidative stress-induced cell death were examined by Proteggente et al. (362). The data obtained indicated that the hesperetin glucuronides, but not hesperetin, protect against UV-A-induced necrotic cell death. As it was recently shown that hesperetin may regulate primary rat osteoblast differentiation through bone morphogenetic protein signaling, the effect of hesperetin-7-O-glucuronide on primary rat osteoblast proliferation and differentiation was investigated by Trzeciakiewicz et al. (461). At a physiological concentration of $1 \mu \mathrm{M}$, the glucuronide did not affect proliferation, but en- hanced differentiation by increasing alkaline phosphatase activity and mRNA expression. It also induced mRNA expression of Runx2 and Osterix, two transcription factors implicated in the regulation of osteoblast functions. Moreover, phosphorylation of Smad1/5/8 was enhanced by the glucuronide, whereas ERK $1 / 2$ remained unchanged after $48 \mathrm{~h}$, and receptor activator of nuclear factor $\kappa$-B ligand (RANKL) gene expression was significantly decreased. These results suggest that hesperetin-7-O-glucuronide may regulate osteoblast differentiation through specific transcription factor stimulation, and might in turn be implicated in the regulation of osteoblast/osteoclast communication.

Tables 12 and 13 represent the synthesis of the molecular actions exerted by (poly)phenolic metabolites in experimental models of CVDs and cancer, respectively. Figures 39 and 40 represent a graphical summary of the main mechanisms of action involved in the cardiovascular and cancer protective effects of some (poly)phenol metabolites, respectively.

\section{Feeding Studies and Evidence of Protective Effects}

There is considerable epidemiological evidence indicating that the consumption of diets rich in fruit and vegetables is associated with a reduction in the risk of a number of chronic diseases, most notably CVD, specific cancers, and neurodegenerative diseases $(24,29,54,90,99,100,137,183,231,264$, $276,375)$. The beneficial effects of such diets have often been attributed to the (poly)phenols they contain. Indeed, associations have also been observed between the dietary intake of (poly)phenols and reductions in the risk of such chronic diseases $(13,83,97,193,309)$. However, to confirm if such observations are genuine, and whether (poly)phenols are indeed responsible for them, well-powered and well-controlled human intervention trials are necessary.

In the last 5-10 years, numerous such trials have been conducted, although arguably, many are flawed, as they lack proper controls, do not provide a detailed compositional analysis of the foods that are being tested, and/or lack a relevant study population. For example, to make sound causal relationships between (poly)phenol intake and the clinical/ physiological outcomes being assessed, a suitable control intervention is essential. A number of studies have strived to utilize such a control food/beverage, which is indistinguishable from the test food/beverage by means of taste and appearance, which is accurately matched for micro- and macronutrient composition, including other potential bioactive compounds.

In this section, we will present data relating principally to the role of (poly)phenols in human health, while considering the points raised above. In particular, we will focus on wellcontrolled, randomized human intervention studies examining the effects of (poly)phenol-rich foods/beverages against CVD, cancer, and neurodegenerative diseases, and/or clinically significant risk factors associated with such disorders.

\section{A. Cardiovascular effects of dietary polyphenols}

There have been a number of short-term, small-scale human intervention studies designed to test the effect of (poly)phenol-rich foods, and in particular those containing flavonoids, on well-characterized, medically significant CVD risk factors, including hypertension, endothelial dysfunction, lipid metabolism, and platelet activation. In this section, we 
Table 12. Mechanisms of Action Exerted by (Poly)phenol Metabolites in Experimental Models Related to Cardiovascular Diseases

\begin{tabular}{|c|c|c|c|c|}
\hline Class & Metabolite & Mechanisms & Model & Reference \\
\hline \multirow[t]{2}{*}{ Anthocyanins } & \multirow[t]{2}{*}{ Protocatechuic acid } & Monocyte adhesion $\downarrow$ & Mouse endothelial cells & 481 \\
\hline & & VCAM1 $\downarrow$, ICAM $1 \downarrow$ & Apo-E mice & 481 \\
\hline \multirow[t]{2}{*}{$\begin{array}{l}\text { Chlorogenic } \\
\text { acids }\end{array}$} & $\begin{array}{l}\text { m-Coumaric acid, } \\
\text { dihydroferulic acid }\end{array}$ & oxLDL $=$ & Chemical LDL oxidation & 155 \\
\hline & $\begin{array}{l}\text { Dihydroferulic acid, } \\
\text { 3-(3'-hydroxyphenyl) } \\
\text { propionic acid }\end{array}$ & $\begin{array}{l}\text { Platelet reactivity } \downarrow \text {, } \\
\text { P-selectin - , NOS } \uparrow\end{array}$ & Platelets & 371 \\
\hline \multirow[t]{2}{*}{ Flavan-3-ols } & $\begin{array}{l}\text { Mixture of plasma } \\
\text { metabolites from rats }\end{array}$ & $\begin{array}{l}\text { Monocyte adhesion } \downarrow \text {, } \\
\text { ROS } \downarrow\end{array}$ & Human aortic endothelial cells & 251 \\
\hline & $4^{\prime}, 4^{\prime \prime}$-Di-O-methyl-EGCG & ICAM1 - & Human aortic endothelial cells & 285 \\
\hline \multirow[t]{4}{*}{ Flavonols } & $3^{\prime}-$ and $4^{\prime}-O$-methyl-quercetin & $\begin{array}{l}\text { ICAM1 - , E-selectin - , } \\
\text { VCAM1 = }\end{array}$ & Human aortic endothelial cells & 285 \\
\hline & $\begin{array}{l}\text { Quercetin-3'-O-sulfate, } \\
\text { quercetin-3-O-glucuronide, } \\
\text { isorhamnetin 3-O-glucuronide }\end{array}$ & $\begin{array}{l}\mathrm{ICAM} 1=, \operatorname{VCAM} 1- \\
\mathrm{MCP} 1=\end{array}$ & HUVECs & 459 \\
\hline & $\begin{array}{l}\text { Quercetin-3'-O-sulfate, } \\
\text { quercetin-3-O-glucuronide, } \\
\text { isorhamnetin 3-O-glucuronide }\end{array}$ & $\begin{aligned} \text { VCAM1 } & =, \text { ICAM1 = } \\
\text { MCP1 } & =\end{aligned}$ & $\begin{array}{l}\text { TNF } \alpha \text {-activated human } \\
\text { artery smooth muscle cells }\end{array}$ & 496 \\
\hline & $\begin{array}{l}\text { Quercetin-3'-O-sulfate, } \\
\text { quercetin-3-O-glucuronide, } \\
\text { isorhamnetin 3-O-glucuronide }\end{array}$ & $\begin{array}{l}\text { Vasorelaxation }=, \mathrm{NO}= \\
\quad \mathrm{NOS}=\end{array}$ & Rat thoracic aortic rings & 282 \\
\hline \multirow[t]{3}{*}{$\begin{array}{l}\text { Lignans, } \\
\text { isoflavones }\end{array}$} & Equol & $\begin{array}{l}\text { Vasorelaxation } \uparrow, \mathrm{NO} \uparrow, \\
\quad \operatorname{eNOS} \uparrow\end{array}$ & Human aortic rings, HUVECs & 232,394 \\
\hline & Equol & PXR $\uparrow$, CYP3A4+ & Human primary hepatocytes & 279 \\
\hline & Equol & eNOS + , relaxation & $\begin{array}{l}\text { Ritonavir-induced eNOS } \\
\text { downregulation and blockage } \\
\text { of BK relaxation }\end{array}$ & 74 \\
\hline
\end{tabular}

$\uparrow$, signaling/effect activation; $\downarrow$, signaling/effect inhibition; $=$, no significant change; + , expression induction (upregulation); - , expression inhibition (downregulation); EGCG, (-)-epigallocatechin-3-O-gallate; HUVECs, human umbilical vein endothelial cells; ICAM-1, intercellular adhesion molecule 1; MCP-1, Monocyte chemotactic protein-1; NO, nitric oxide; NOS, nitric oxide synthase; oxLDL, oxidated low-density lipoprotein; PXR, pregnane X receptor; ROS, reactive oxygen species; TNF- $\alpha$, tumour necrosis factor- $\alpha$; VCAM- 1 , vascular cell adhesion molecule 1.

report on human randomized controlled trials (RCTs), where at least one of these markers has been assessed (see Table 14). The following (poly)phenol-rich foods will be discussed: cocoa, red wine, berries, pomegranate, tea, coffee, citrus fruits, and nuts, although the amount and quality of the data vary.

1. Cocoa. There is good evidence to suggest that cocoaderived (poly)phenols may have beneficial effects on blood pressure (BP) in healthy subjects and, in particular, hypertensive individuals $(89,112,146,165,166,168,211,325,326$, $453,454)$. A meta-analysis of 10 RCTs comprising healthy, normotensive, pre-, and hypertensive individuals reported a decrease of $4.5 \mathrm{~mm} \mathrm{Hg}$ in systolic BP and $2.5 \mathrm{~mm} \mathrm{Hg}$ in diastolic BP after intake of flavan-3-ol-rich chocolate or flavan3-ol-rich cocoa for 2-18 weeks (112). However, caution should be expressed over the fact that seven of the studies used in the meta-analysis were noted to have used white chocolate, which is very different in appearance, taste, and nutrient content from dark chocolate, as a control for the high-flavan3-ol test intervention. A more recent meta-analysis of 20 randomized controlled studies concluded that systolic BP decreased by $1.63 \mathrm{~mm} \mathrm{Hg}$, whereas diastolic BP and heart rate were unaffected after the consumption of flavan-3-ol-rich cocoa (421). Looking closely at these meta-analyses, it is evident that there are large differences in the quality of the selected studies.
With regard to endothelial function, a well-known surrogate marker for vascular health, flavan-3-ol-rich cocoa (176$963 \mathrm{mg}$ of flavan-3-ol monomers and oligomers), has been shown to acutely improve endothelium-dependent vasodilation assessed by flow-mediated dilation (FMD) via increasing plasma NO bioavailability in healthy individuals and in patients with coronary artery disease (CAD), hypertension, or diabetes, compared to a well-matched control containing 0$75 \mathrm{mg}$ flavan-3-ols $(22,102,136,187,188,190,404)$. These vascular improvements correlated in time with changes in plasma flavan-3-ol metabolites, and were repeatable with pure (-)-epicatechin intake, suggesting a cause-and-effect relationship between flavan-3-ols and vascular improvements (404). Flavan-3-ol-rich cocoa has also been shown to reverse endothelial dysfunction in smokers (190) and to improve endothelium-dependent coronary vasomotion in heart transplant recipients (143).

Short-term flavan-3-ol-rich cocoa intake has also been shown to increase the FMD response and hyperemic brachial artery blood flow after 2-12-week consumption in obese, hypercholesterolemic, hypertensive, and healthy subjects $(102,131,165,168,333,486)$. A sustained increase in baseline FMD levels and an additional acute-on chronic increase in the FMD response were also reported in, respectively, healthy smokers and diabetics, after 7 and 30 days of flavan-3-ol-rich cocoa consumption $(22,188)$. Nine studies on cocoa and endothelial function were included in the meta-analysis of 
Table 13. Mechanisms of Action Exerted by (Poly)phenol Metabolites in Experimental Models Related to Cancer

\begin{tabular}{|c|c|c|c|c|}
\hline Polyphenols & Metabolites & Mechanisms & Model/cell line & Reference \\
\hline \multirow[t]{5}{*}{ Anthocyanins } & Protocatechuic acid & $\begin{array}{l}\text { Apoptosis } \downarrow \text {, ROS } \downarrow \text {, } \\
\text { caspases } \downarrow\end{array}$ & Neural stem cells & 172 \\
\hline & & $\begin{array}{l}\text { Apoptosis } \uparrow, \mathrm{JNK} \uparrow, \\
\text { MAPK } \uparrow\end{array}$ & Gastric adenocarcinoma & 280 \\
\hline & & $\begin{array}{l}\text { Proliferation } \downarrow \text {, JNK } \uparrow \text {, } \\
\quad \text { MAPK } \uparrow\end{array}$ & HepG2 & 508 \\
\hline & & $\begin{array}{l}\text { ROS } \downarrow \text {, caspase } 3 \downarrow \text {, } \\
\text { Bcl }-,\end{array}$ & PC12 & 173 \\
\hline & Various & Proliferation $\downarrow$ & $\mathrm{Caco} 2$ & 144 \\
\hline \multirow[t]{5}{*}{ Ellagitannins } & Urolithin A & Wnt $\downarrow$ & $293 \mathrm{~T}$ & 414 \\
\hline & Urolithin A & Proliferation $\downarrow$ & LAPC-4 & 406 \\
\hline & Various urolithins & EROD $\downarrow$, CYP1B1 - & 22Rv1, prostate cancer & 241 \\
\hline & Urolithin A and B & $\begin{array}{l}\text { Aromatase } \downarrow \text {, } \\
\quad \text { proliferation } \downarrow\end{array}$ & MCF7aro & 1 \\
\hline & Pyrogallol & EphA2 $\downarrow$ & PC3 & 457 \\
\hline Flavan-3-ols & M4, M6 & Proliferation $\downarrow$ & Various cancer cells & 266 \\
\hline \multirow[t]{3}{*}{ Flavonols } & Quercetin-3-O-glucuronide & VEGF $\downarrow$, ERK $\downarrow$ & $\begin{array}{l}\text { Bovine coronary venular } \\
\text { endothelial cells }\end{array}$ & 117 \\
\hline & Quercetin-3'-O-sulfate & VEGF $\uparrow$, ERK $\uparrow$ & $\begin{array}{l}\text { Bovine coronary venular } \\
\text { endothelial cells }\end{array}$ & 117 \\
\hline & $\begin{array}{l}\text { Quercetin-3'-O-sulfate } \\
\text { Quercetin-3-O-glucuronide }\end{array}$ & Angiogenesis $=$ & Rabbit cornea & 117 \\
\hline \multirow[t]{3}{*}{$\begin{array}{l}\text { Lignans, } \\
\text { isoflavones }\end{array}$} & Enterolactone & $\begin{array}{l}\text { Proliferation } \downarrow \text {, } \\
\quad \text { apoptosis } \uparrow\end{array}$ & LNCaP & 73 \\
\hline & Enterolactone & $\mathrm{ER} \alpha+$, proliferation $=$ & MCF7 & 64 \\
\hline & Enterodiol & $\begin{array}{l}\mathrm{ER} \alpha+, \operatorname{ER} \beta+, \\
\text { proliferation } \uparrow\end{array}$ & MCF7 & 64 \\
\hline
\end{tabular}

$\uparrow$, signaling/effect activation; $\downarrow$, signaling/effect inhibition; =, no significant change; + expression induction (upregulation); - , expression inhibition (downregulation); Bbl2, B-cell lymphoma 2; ER, estrogen receptor; ERK, extracellular signal-regulated kinases; EROD, CYP1B1mediated ethoxy resorufin-O-de-ethylase assay; M4, 5-(3',4',5'-trihydroxyphenyl)- $\gamma$-valerolactone; M6, 5-(3', 4'-dihydroxyphenyl)- $\gamma$-valerolactone; MAPK, mitogen-activated protein kinase.

Shrime et al. (421), which concluded that flavan-3-ol-rich cocoa increased FMD in average by $1.53 \%$. A dose-dependent effect was also observed with FMD increasing up to a $500 \mathrm{mg}$ intake of flavan-3-ols and decreasing at higher doses. In the same meta-analysis, consumption of flavonoid-rich cocoa was also found to increase high-density lipoprotein (HDL) cholesterol by $0.046 \mathrm{mM}$ and decrease LDL cholesterol (LDL-C) by $0.07 \mathrm{mM}$. An indication of dose dependency between the flavan-3-ol and HDL cholesterol (HDL-C) was also observed. Furthermore, subgroup analysis revealed that HDL-C tended to increase in longer studies with low-fat consumption, and LDL and total cholesterol tended to decrease in shorter studies, in studies with younger populations, and in trials with high-fat consumption (421).

The effects of cocoa flavan-3-ol intervention on a number of other CVD risk factors have also been investigated. For example, there is good evidence to suggest that they may improve insulin resistance $(102,165,166,168)$. Notably, after $2-$ 12 weeks of cocoa flavan-3-ol intake of 500-1000 mg/day, a decrease of 0.94 points in the homeostasis model assessment of insulin resistance and a decrease of 4.95 points in the insulin sensitivity index were observed. However, there were no changes in the quantitative insulin sensitivity check index and fasting glucose levels. Together, these results suggest that cocoa intake may improve insulin sensitivity in healthy, hypertensive, and obese individuals (421). There are also extensive data regarding the effects of cocoa intake on platelet function $(209,326,350,373)$. Inhibition of platelet activation has been observed at 2 and $6 \mathrm{~h}$ post-flavan-3-ol-rich cocoa/ chocolate consumption in healthy individuals $(209,373)$. Such acute observations are supported by chronic intervention studies where cocoa flavan-3-ols improve platelet function after 28 days of supplementation (326). Although less effective, an aspirin-like effect for flavan-3-ols has been suggested, in inhibiting platelet activation (350).

2. Red wine. It is well accepted that excessive alcohol consumption leads to increased BP in normotensive and hypertensive subjects $(364,365)$, whereas low/moderate consumption reduces specific cardiovascular risk factors (51, 384). Reports regarding the effects of red wine and red wine (poly)phenols on BP are in most case inconsistent. While there is extensive evidence to support the influence of wine intake on cardiovascular health $(86,116,281)$, controversy remains whether red wine in particular exerts beneficial effects compared with other alcoholic beverages $(47,469)$ or simply alleviates the detrimental influence of alcohol on BP $(55,512)$. In acute studies with healthy volunteers, no changes in BP were observed, but an increase in the heart rate was reported after red wine consumption $(181,427)$, whereas in CAD patients, a decrease in systolic and diastolic BP was noted together with an increase in heart rate, $1 \mathrm{~h}$ postwine (red and white) intake (489). Other studies have reported no changes in hemodynamics or BP after medium-term daily intake of either red 


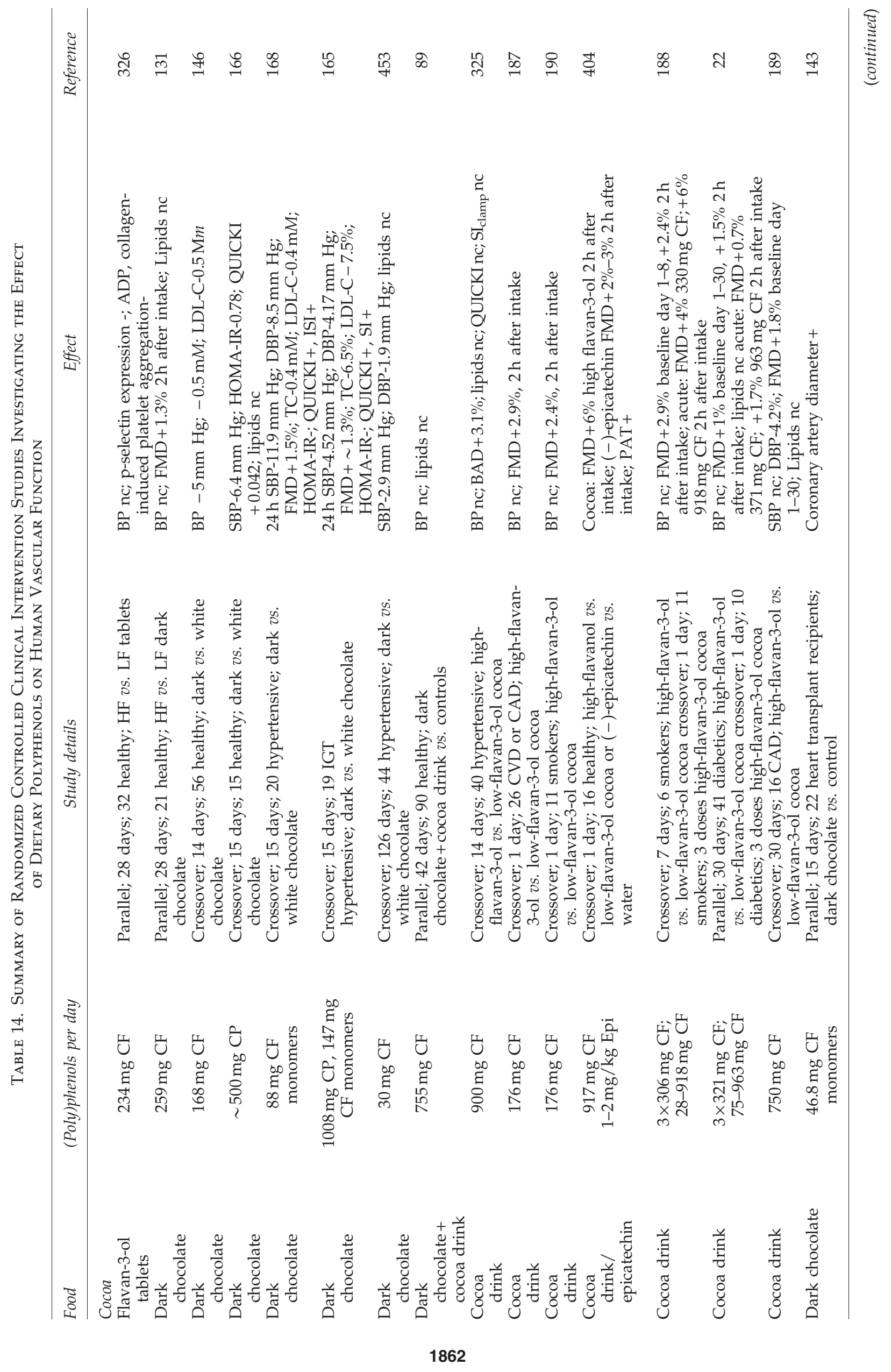




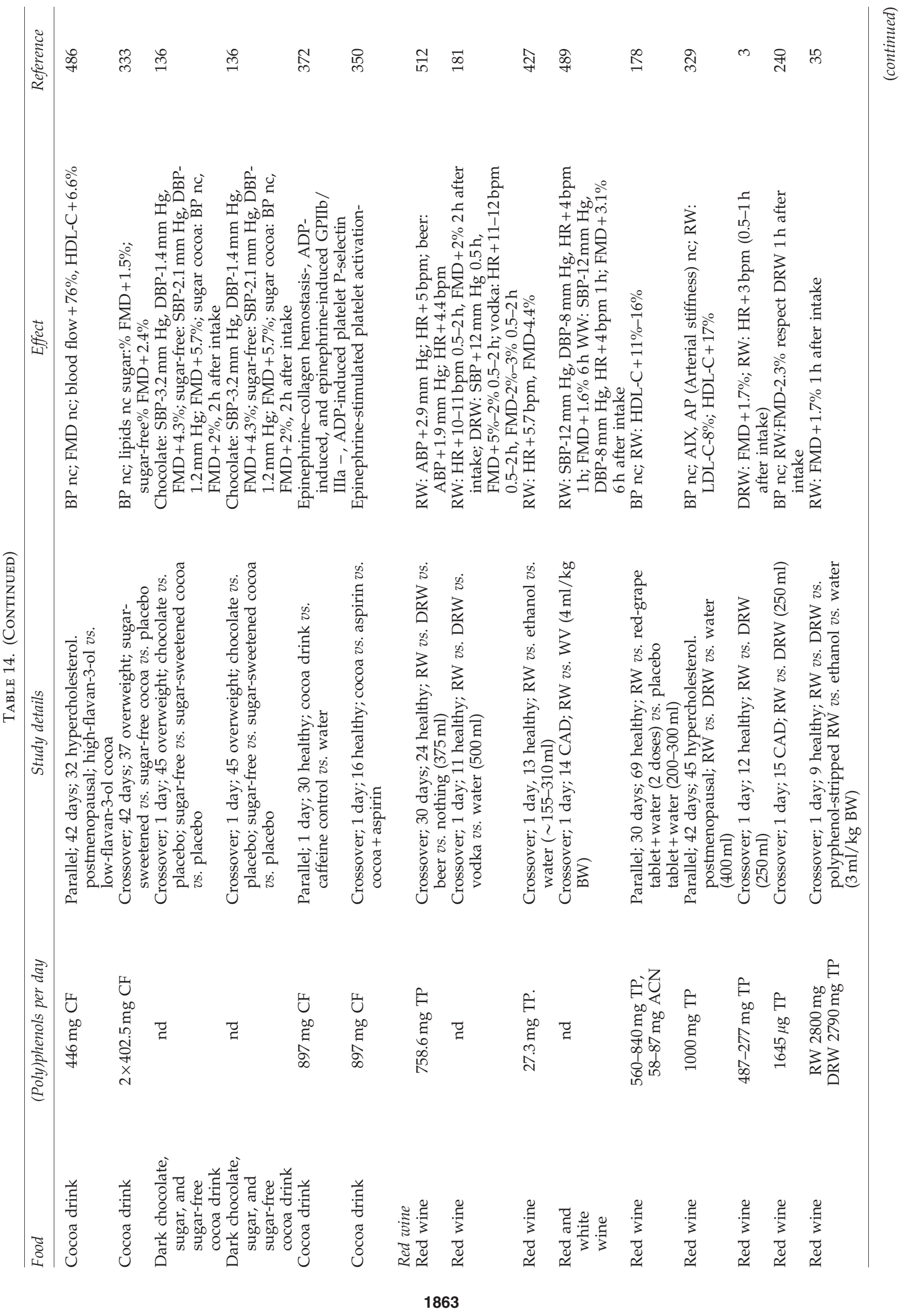




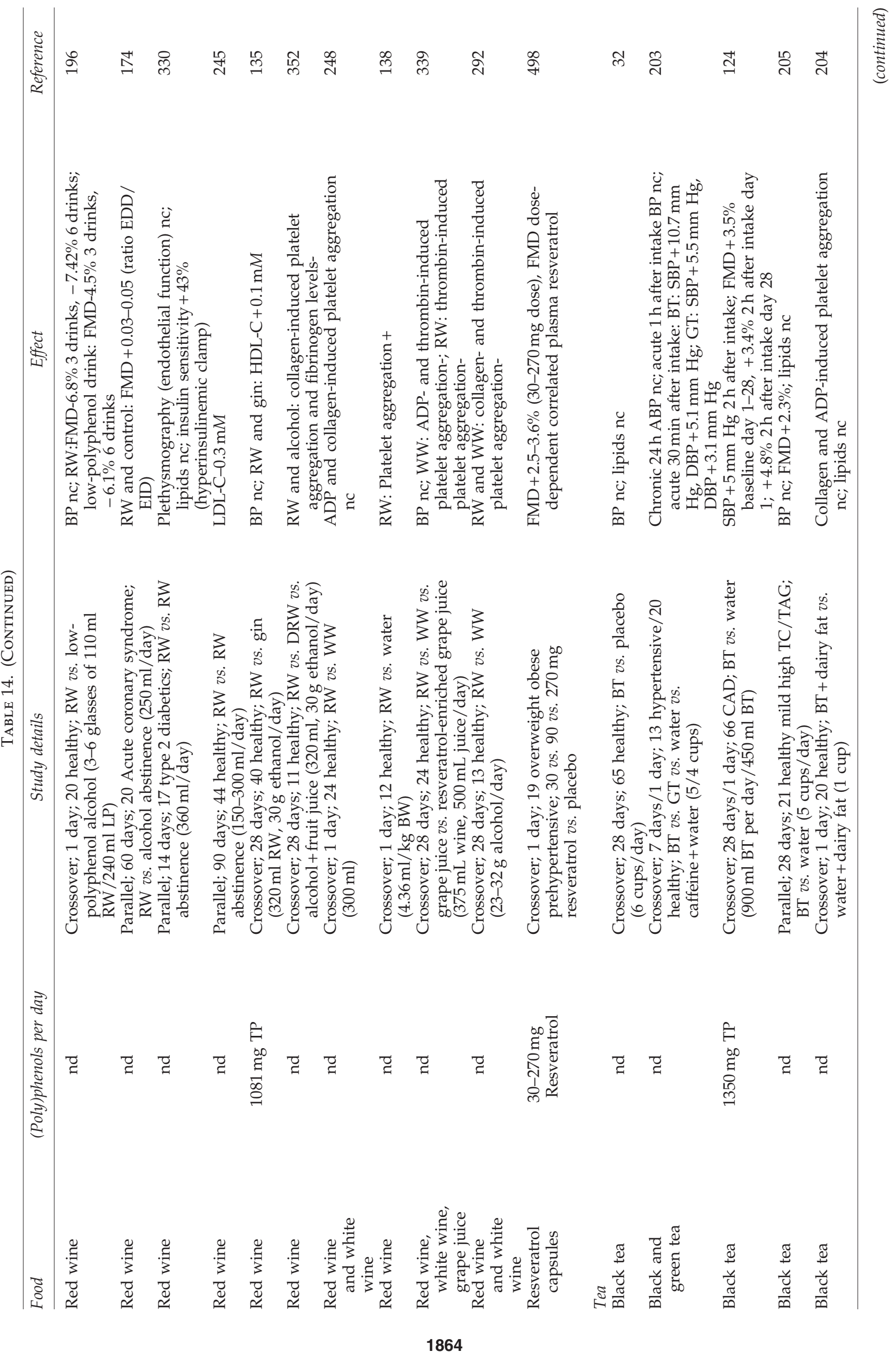




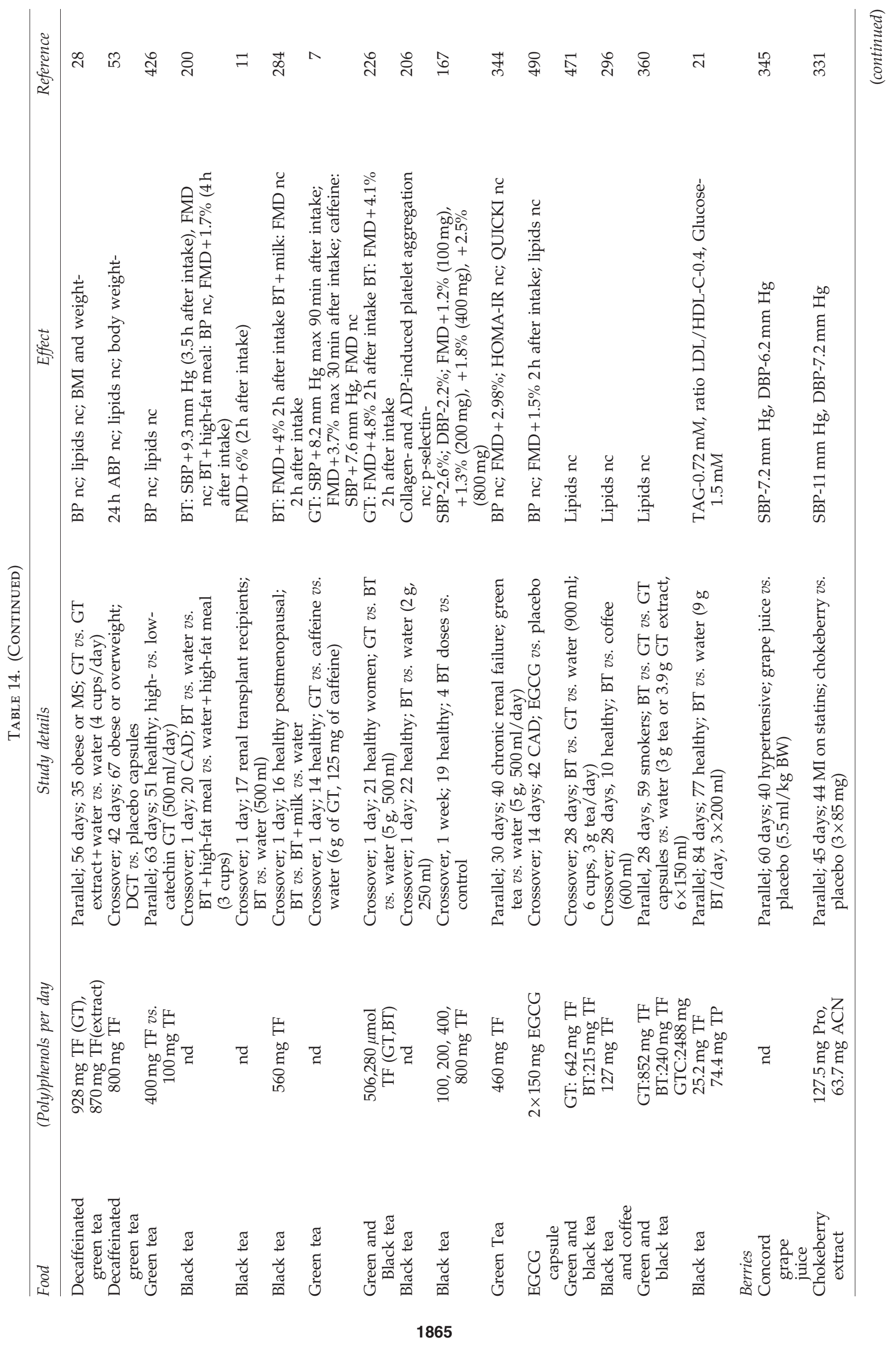




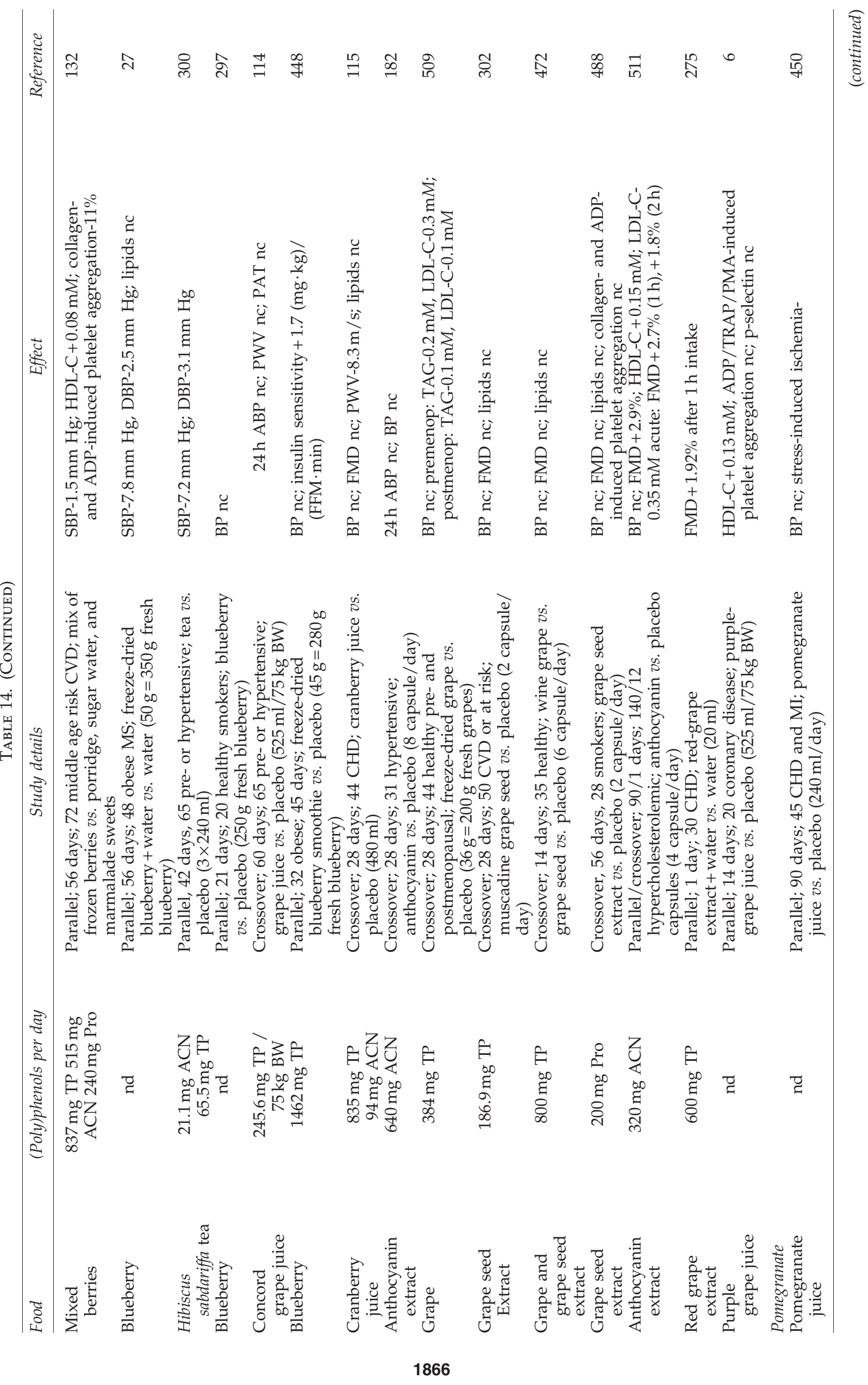




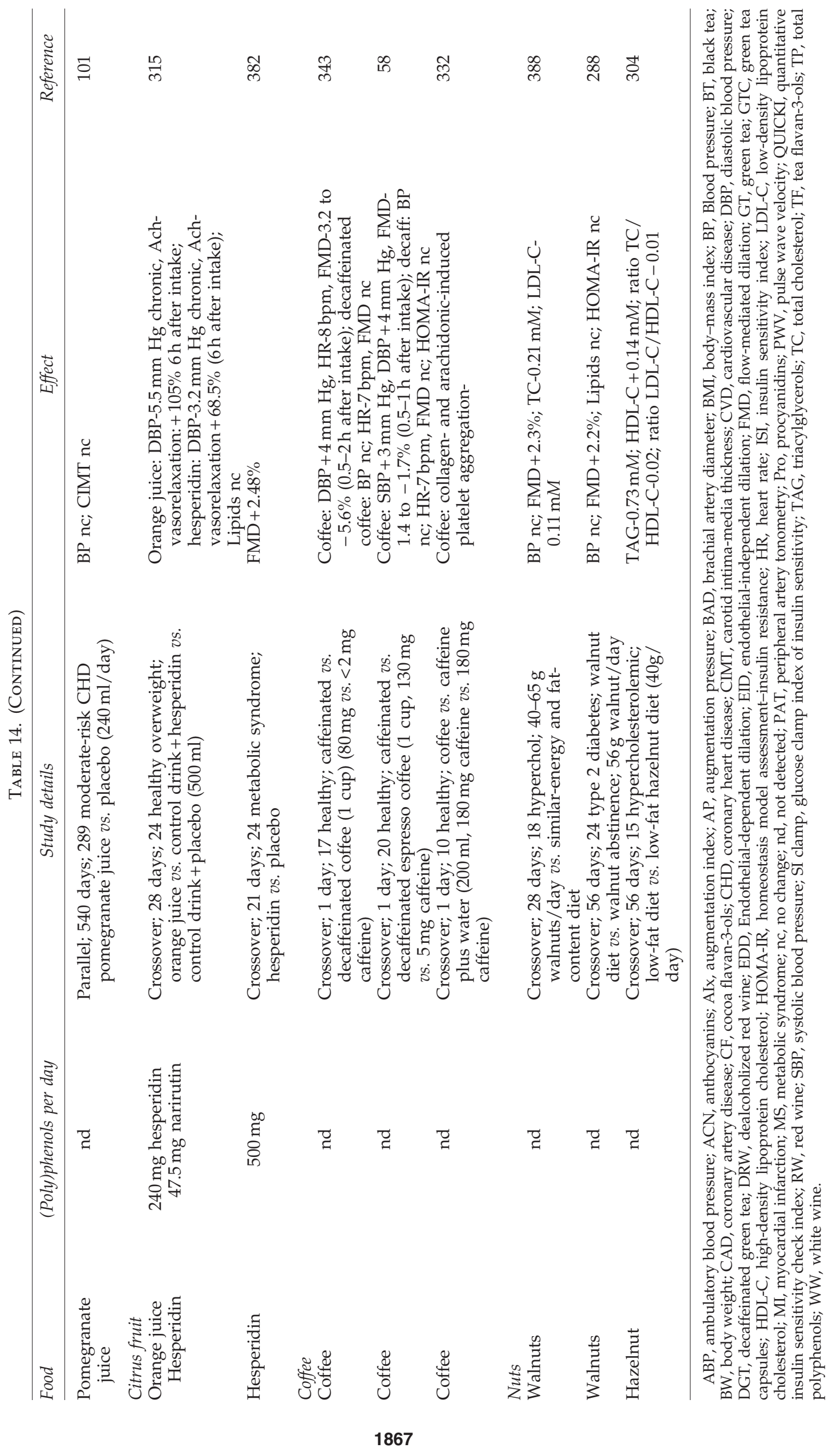


wine or its dealcoholized equivalent $(178,329)$. However, in the majority of these studies, no proper control was used.

Several studies report the effect of red wine and/or its dealcoholized equivalent on endothelial function, although again, there is inconsistency in the findings. For example, some studies indicate that dealcoholized wine, but not red wine, induces an increase in FMD $(3,240)$; others suggest the opposite (35); and another suggests that both red wine and dealcoholized red wine are equally effective (181). Other studies have shown no effect, or even a decrease, in FMD after red wine or alcohol consumption $(196,427)$. The reason for these inconsistencies may be the increase in the baseline brachial artery diameter due to alcohol reported in all the studies, which will affect FMD responses, as pointed out by Spaak et al. (427). With regard to longer-term (2 weeks to 2 months) red wine consumption, the majority of studies suggest that there is no significant effect on FMD or arterial stiffness (174, $329,330,512)$.

In addition to BP and endothelial function, several studies have also reported that regular medium-term intake of red wine (2-12 weeks) may increase HDL-C and lower LDL-C $(178,245,328,378)$. As there were no controls used in these studies with alcohol, it remains unclear as to whether the alcohol itself, rather than (poly)phenols, was responsible for the favorable effects of red wine. Indeed, HDL-C has been found to increase after a 28-day intake of either red wine or gin (135). Acute studies have failed to show a positive effect on platelet function after red wine consumption $(138,248)$. However, red wine, dealcoholized red wine, white wine, and alcohol all appear to have the same beneficial effects on platelet function after 4 weeks of intake $(292,339,352)$, which suggests that the positive effects of moderate consumption of wines on platelet function seem to be due to their alcohol content.

Resveratrol (Fig. 13), a stilbene found in grape seed, has been postulated to be partly responsible for the beneficial vascular effects of red wine. Indeed, 30, 90, or $290 \mathrm{mg}$ of transresveratrol has been shown to improve endothelial function in overweight subjects in a dose-dependent manner (498), and animal studies support this, as resveratrol reportedly improves survival rates, endothelium-dependent smooth muscle relaxation, cardiac contractile, and mitochondrial function in a hypertensive model of heart failure (379). However, while the conjecture that resveratrol may underpin the vasoactive effects of wine seems logical, the majority of red wines actually contain very little of the stilbenes, and thus it is unlikely that it is responsible for the beneficial effects of red wine in vivo (see Section III.J)

3. Tea. Tea is also a rich source of flavan-3-ols; although unlike those found in cocoa and red wine, they are predominantly gallated (Fig. 9). As with wine, there is some controversy regarding the effects of tea on $\mathrm{BP}$, with the presence of caffeine likely to confuse the issue if studies are not properly controlled $(211,301)$. Meta-analysis (211) has indicated that acute, but not chronic, black tea intake affects systolic and diastolic BP in healthy subjects. In agreement with this, regular consumption of both green and black tea (1-9 weeks) have been shown not to significantly influence BP $(28,32,123$, 203, 204, 426, 454)

With regard to endothelial function, the meta-analysis by Hooper et al. (211) indicates that black tea consumption significantly increases the FMD response after acute $(+1.7 \%)$ and long-term consumption $(+3.4 \%)$, whereas that of Ras et al. (369), reporting on nine RCTs, concluded that moderate consumption of black (seven studies) and green (three studies) tea $(2-3 \times 500 \mathrm{ml}$ cups/day) improved FMD by $2.6 \%$. Subgroup analysis indicated that FMD increases occurred in healthy, diseased, young, and old subjects, and in acute $(7,11$, $123,200,226,284)$ and short-term consumption (1-4 weeks) $(123,167,204,344)$ studies in response to both green and black tea. As with in the case of cocoa flavan-3-ols, long-term tea consumption has been shown to produce a sustained increase in baseline FMD levels and an additional acute-on chronic increase in the FMD response (123). Acute intervention with the tea flavan-3-ol, (-)-epigallocatechin-3-O-gallate, also led to improvements in endothelial function, suggesting that the gallate may be partly responsible for the vascular effects of tea (490).

With regard to other risk factors for CVD, many human intervention studies have indicated that neither green nor black tea intake has an effect on blood lipids $(32,296,360,426$, 471). However, a recent study with healthy individuals has reported that black tea decreases triglyceride levels by $35.8 \%$ and the LDL/HDL plasma cholesterol ratio by $20.3 \%$ after 12 weeks of consumption (21). Human intervention studies in both healthy and CAD subjects indicate no effect on platelet aggregation $(124,204,206)$. In contrast, a decrease in platelet activation has been reported in healthy men after a 6-week consumption of black tea (443), whereas 4 weeks of consumption brought about a decrease in p-selectin, a marker of platelet activation (206).

4. Berries. A decrease in $\mathrm{BP}$ has been reported after chronic intakes (6-8 weeks) of mixed berries, anthocyaninrich tea, and chokeberry and blueberry extracts by hypertensive individuals, myocardial infarction survivors, and those with markers of the metabolic syndrome $(27,132,300$, $331,345)$. In contrast, a similar number of studies have reported a lack of efficacy of chronic intake of anthocyanin-rich foods, such as blueberry and cranberry juice in healthy individuals, chronic smokers, people with dyslipidemia, obese subjects, stage 1 hypertensive individuals, and CAD patients $(114,115,182,297,366,448)$. Similarly, grapes and grape seed extract have also been shown not to influence BP in healthy or diseased individuals $(302,472,488,509)$. Animal studies have been more positive, showing BP-lowering effects of berry extracts after long-term consumption, but only in models of hypertension $(130,415,497)$. There is no evident reason on why some studies show a positive effect on BP and others not, although different types of berries may contain very different profiles of (poly)phenols (41).

Improvements in vascular function (increased FMD) have been reported acutely with pure anthocyanins (320 mg) (511), after consumption of a grape extract $(72,275)$ administered with or without a high-fat meal (72), and after consumption of cranberry juice (4 weeks; decrease in carotid femoral pulse wave velocity) (115). Despite these promising results, other studies have failed to observe a positive effect on endothelial function after consumption of grapes $(302,472,488)$. Berry and grape consumption has also been shown to lead to increases in HDL-C $(6,132,366,511)$ and decreases in LDL-C (509) in healthy, at risk, and diseased individuals. Others have reported no changes in blood lipids despite observing changes in other CVD markers $(72,114,115,127,302,472)$. The 


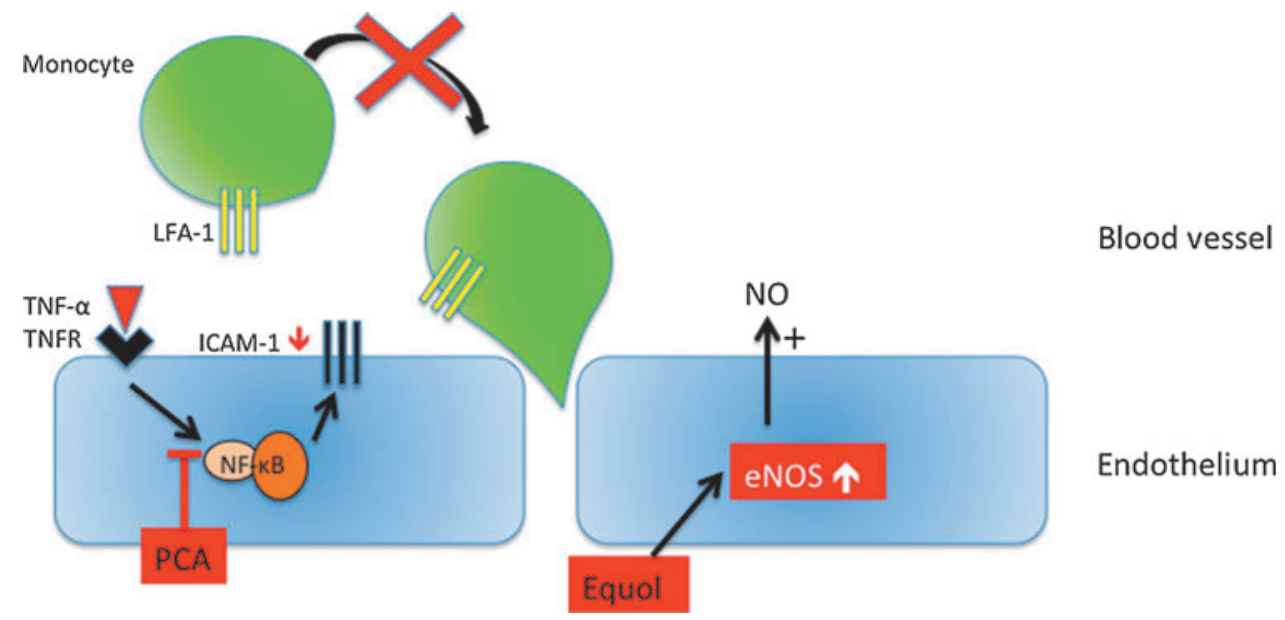

FIG. 39. Graphical summary of some of the mechanisms involved in cardiovascular protection exerted by (poly)phenol metabolites. Protocatechuic acid (3,4-dihydroxybenzoic acid) impairs monocyte adhesion by inhibiting the nuclear content of p65, a subunit of NF- $\kappa$ B, and thus decreasing ICAM-1 and VCAM-1 expression. Moreover, equol induces eNOS expression by increasing NO production. eNOS, endothelial nitric oxide synthase; ICAM-1, intercellular adhesion molecule 1; LFA-1, lymphocyte function-associated antigen-1; NF- $\kappa$, nuclear factor kappa-light-chain enhancer of activated B cells; NO, nitric oxide; PCA, protocatechuic acid; TNF- $\alpha$, tumor necrosis factor- $\alpha$; TNFR, TNF- $\alpha$ receptor. (To see this illustration in color, the reader is referred to the web version of this article at www.liebertpub.com/ars.)

evidence for berries, including Concord grape juice, inhibiting platelet aggregation is positive $(6,132,357)$, and recent evidence suggests that berry consumption may improve insulin sensitivity (448).

5. Pomegranate. Pomegranate has been suggested to have antiatherosclerotic effects in human studies and animal models of disease $(17,19,101,239,389,450)$. The consumption of pomegranate juice (3 months, $240 \mathrm{ml}$ of pomegranate juice/ day) has been reported to lead to significant decreases in stress-induced ischemia, assessed by myocardial perfusion imaging at rest and during stress (treadmill test exercise) with which may decrease myocardial ischemia and improve myocardial perfusion in patients with coronary heart disease

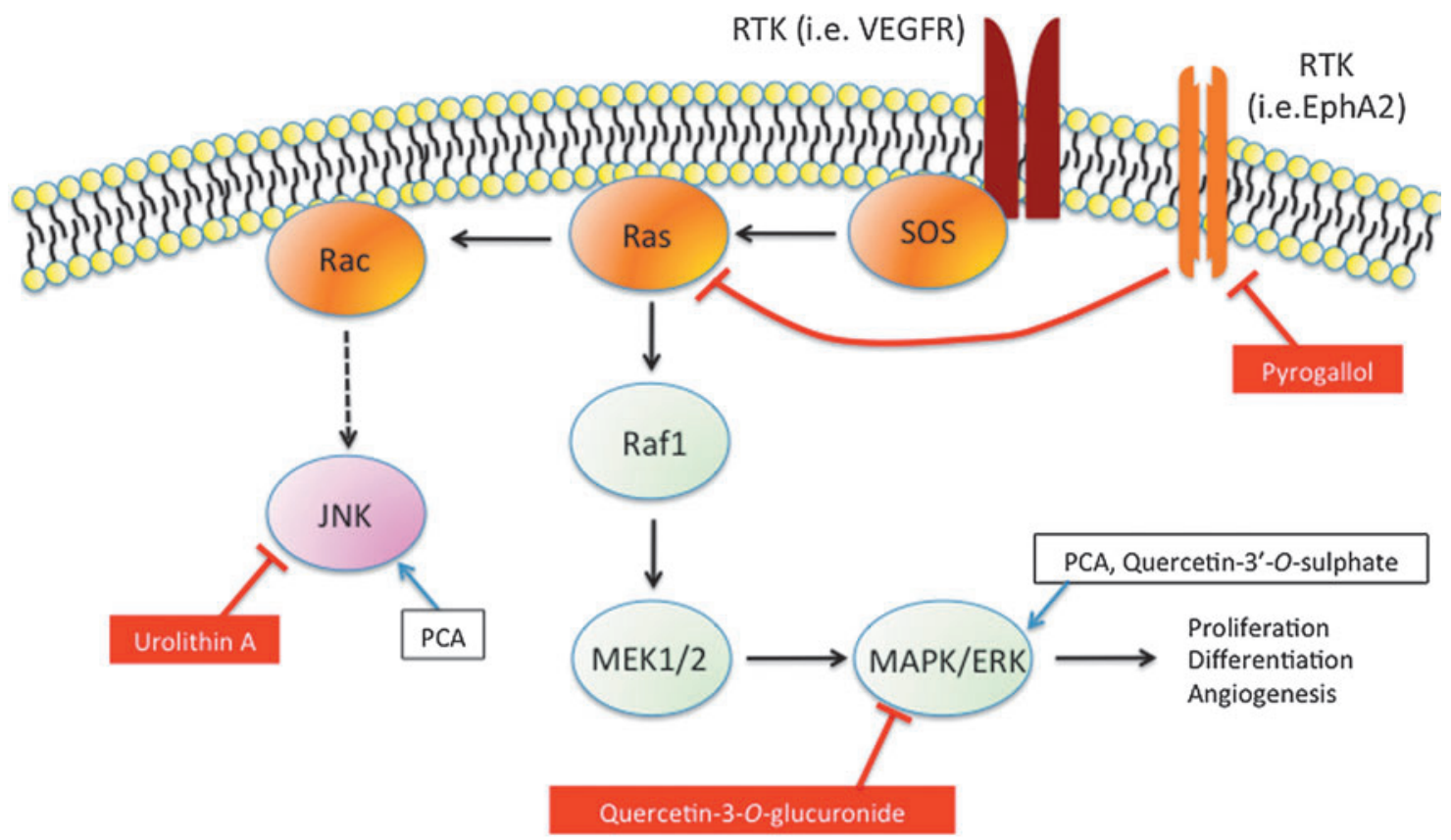

FIG. 40. Graphical summary of some of the mechanisms involved in pathways related to cancer. MAPK/ERK signaling is inhibited by quercetin-3-O-glucuronide and enhanced by PCA and quercetin-3'-O-sulfate. JNK activity is reduced by urolithin A and increased by protocatechuic acid. Pyrogallol (1,2,3-trihydroxybenzene) inhibits EphA2 kinase activation, interfering with ephrinA1 binding (see also Fig. 39). Arrows identify activation interactions, whereas truncated lines classify inhibition interactions. Dotted lines are used to simplify transduction pathways. ERK, extracellular signal-regulated kinases; JNK, c-Jun N-terminal kinase; MAPK, mitogen-activated protein kinase; RTK, receptor tyrosine kinase; VEGFR, vascular endothelial growth factor receptor. (To see this illustration in color, the reader is referred to the web version of this article at www.liebertpub.com/ars.) 
(CHD) (450). Furthermore, daily intake of $240 \mathrm{ml}$ of pomegranate juice for 18 months improved carotid intima-media thickness (CIMT) of at risk individuals relative to a control (similar characteristics, color, and energy), although only in those with the most adverse tertiles for blood lipids (101). Although an uncontrolled intervention study (i.e., the control was no consumption), a daily $50-\mathrm{ml}$ consumption of pomegranate juice for 12 months has also been shown to induce a $12 \%$ decrease in systolic BP and a 30\% decrease in CIMT in patients with carotid artery stenosis (18).

Many animal investigations have supported these these reported cardiovascular effects in humans $(17,19,239,389)$. In an atherosclerotic mouse model, a 3-month supplementation with pomegranate juice, pomegranate fruit liquid extract, pomegranate (poly)phenol powder extract, and, in particular, a pomegranate ground flower extract led to significant decreases in the atherosclerotic lesion area relative to water-fed controls along with decreases in serum glucose and cholesterol levels (19). Furthermore, intervention with pomegranate juice extract for 4 weeks reduced the mean arterial BP, decreased the vascular reactivity in response to catecholamines, and reversed biochemical changes induced by angiotensin II in diabetic rats (311). Further, better-controlled human and animal studies are required to conclude on the likely efficacy of pomegranate against CVD.

6. Citrus fruit. Medium-term (4-5 week), daily consumption of flavanone-rich grapefruit (374) and orange (315) juice has been shown to induce decreases in diastolic BP. In support of these findings, consumption of pure hesperidin, $500 \mathrm{mg} /$ day for 3 weeks, resulted in significant increases in the FMD response compared with a placebo (382). Also, both orange juice and hesperidin significantly improve acetylcholine-mediated vasodilation $6 \mathrm{~h}$ after ingestion (315). Unlike endothelial function, no changes in plasma lipids were observed after intakes of either orange juice or pure hesperidin (315), although one study suggests that consumption of $750 \mathrm{ml}$, but not 250 or $500 \mathrm{ml}$ of orange juice, may significantly increase HDL-C (21\%) and triacylglycerol (30\%) and decrease the LDL/HDL cholesterol ratio by $16 \%$ in hypercholesterolemia patients (263). Although the evidence is limited, the fact that two studies have utilized purified flavonoids provides additional weight to previous investigations $(404,490)$, which suggest that flavonoids themselves are the causal agents in mediating vascular benefits.

7. Coffee. A meta-analysis of 11 intervention studies regarding coffee consumption reports that coffee intake increases systolic BP by $2.4 \mathrm{~mm} \mathrm{Hg}$ and diastolic BP by $1.2 \mathrm{~mm}$ $\mathrm{Hg}$ (224). A correlation between the number of cups of coffee consumed and the increase in BP was observed, and the effect of coffee on BP was reported to be greater in studies with younger populations. These findings are supported by another meta-analysis of 16 RCTs examining coffee and caffeine intake for at least 7 days (334). Here, an increase of $1.22 \mathrm{~mm}$ $\mathrm{Hg}$ in systolic BP and $0.49 \mathrm{~mm} \mathrm{Hg}$ in diastolic BP was observed in response to coffee consumption. However, in studies where caffeine on its own was supplemented, increases in BP were higher $(4.16 \mathrm{~mm} \mathrm{Hg}$ for systolic and $2.41 \mathrm{mg} \mathrm{Hg}$ for diastolic BP), suggesting that coffee (poly)phenols, most notably chlorogenic acids (see Section II.B), may limit the negative effects of caffeine on BP. A more recent meta-analysis, looking at hypertensive subjects, reports an acute increase in BP $3 \mathrm{~h}$ after ingestion of $200-300 \mathrm{mg}$ of caffeine, but no significant effect for longer-term consumption (305). Indeed, analysis of cohort studies provides no evidence of an association between habitual coffee consumption and a higher risk of CVD in hypertensive individuals (305). Indeed, long-term, moderate coffee consumption has been associated with a lower risk of CHD in women (503).

In healthy subjects, acute coffee intake (one cup) led to a decrease in the FMD response, with no effect on endothelial function reported for decaffeinated coffee $(58,343)$. However, if the dose of decaffeinated coffee is increased, an improved FMD response may be measured (59), suggesting that coffee (poly)phenols are able to improve acute endothelial function. Caffeinated coffee intake appears to elevate serum lipids and cholesterol in a dose-dependent manner in patients with hyperlipidemia (223), whereas coffee consumption, but not caffeine, has been found to decrease platelet aggregation acutely (332).

8. Nuts. Whole, unprocessed nuts are rich in flavan-3-ols, flavonols, anthocyanins, and, most notably, procyanidins and phenolic acids (37). A recent review of 19 RCTs concluded that consumption of nuts has no effect on BP (66). However, the majority of these studies were uncontrolled, and BP was often a secondary outcome measure. Improvements in FMD have been observed after 4 weeks of walnut or pistachio consumption in healthy, hypercholesterolemia, or type 2 diabetes patients compared to the same diet without nuts $(288,388$, 401). In contrast, hazelnuts ( $40 \mathrm{~g} /$ day; 4 weeks) failed to elicit an FMD effect (304). Studies with nuts, perhaps expectedly, have given more emphasis to plasma lipid changes. A metaanalysis considering 13 studies concluded that consumption of walnuts for periods of 4-24 weeks is capable of decreasing total and LDL-C, whereas HDL-C and triglycerides were unaffected (23), while another systematic review reported a decrease in total and LDL-C after short-term consumption of almonds, peanuts, pecan nuts, and walnuts, with human studies of 2-3-week duration (316). Whether or not these changes are due to the (poly)phenols found in nuts is presently unclear, although it seems likely that unsaturated fats, mainly oleic and linoleic acids, may contribute to lipid-lowering effects in vivo.

\section{B. (Poly)phenols and neurodegenerative diseases}

A combination of preclinical and epidemiological studies suggests that (poly)phenols may be effective in reversing neurodegenerative pathology and age-related declines in neurocognitive performance, although at present, a direct association between (poly)phenol consumption and improvement in neurological health has not been made. The potential of (poly)phenols to improve neurological health appears to be related to a number of mechanisms, including their ability to interact with intracellular neuronal and glial signaling, to influence the peripheral and cerebrovascular blood flow, and to reduce neuronal damage and losses induced by neurotoxins and neuroinflammation $(148,327,424,428,430-432,492)$. This section will examine the beneficial effects of flavonoids on memory, learning, and neurocognitive performance.

There are a number of studies that have indicated that the consumption of flavonoid-rich plant foods and extracts may 
also be capable of inducing improvements in cognitive performance $(83,276,429,431)$. Human observational and interventional studies have hinted at the potential benefits of such foods and their regular intake [reviewed in (289)]. The efficacy of isoflavones on cognition and aspects of memory are well reported $(67,122,141,145,198,214,255,259)$, and may relate to their potential to mimic the actions of estrogens in the brain $(33,191)$, or to influence the synthesis of acetylcholine and neurotrophic factors $(341,342)$. With regard to other flavonoids, human data are somewhat scarcer. Observational data have suggested that the regular and moderate intake of flavonoid-rich foods, such as wine, berries, and cocoa, may result in cognitive benefits $(83,276,335)$. In addition, two intervention studies have examined the effects of flavonoid-rich extracts of Ginkgo biloba in older adults with (274) and without (310) Alzheimer's disease (AD); two detailed the effects of procyanidin-rich pine bark extracts on healthy older adults $(355,397)$; and one examined the effects of flavan-3-ol-rich cocoa on cognitive function in young healthy female adults (149).

In terms of more commonly consumed flavonoid-rich foods, intervention trials have shown that grape juice, blueberry, and cocoa intake results in positive effects on cognitive outcome measures $(257,258,403)$, although there are studies that suggest no changes in neurophysiological effects (89). With respect to berries, there is a large body of animal behavioral evidence that supports the efficacy of flavonoids on memory and learning. Grape, pomegranate, strawberry, and blueberry $(1 \%-2 \%$ [w/w] freeze-dried fruit/fruit juice), as well as pure (-)-epicatechin and quercetin, have been shown capable of affecting several aspects of memory and learning, notably rapid (484) and slow $(180,229,230,416,495)$ memory acquisition, short-term working memory $(207,363,368,484$, $491)$, long-term reference memory $(65,179)$, reversal learning $(207,484)$, and memory retention/retrieval (473). For example, there is extensive animal evidence suggesting that blueberries are effective at reversing age-related deficits in spatial working memory $(9,26,65,368,383,423,491,494)$. In addition, (-)-epicatechin at a dose of $500 \mu \mathrm{g} / \mathrm{g}$ enhances the retention of rat spatial memory, especially when combined with exercise (473). These observations suggest that flavonoids may be causal agents in inducing the behavioral effects. In addition, two intervention studies have examined the effects of flavonoid-rich extracts of G. biloba in older adults with (274) and without (310) AD; two detailed the effects of procyanidinrich pine bark extracts on healthy older adults $(355,397)$; and one examined the effects of flavan-3-ol-rich cocoa on cognitive function in young healthy female adults (149). While these data suggest an effect of flavonoids in preventing cognitive impairment, at this time, it is not possible to conclude on cause-and-effect relationships between these components and biological effects.

Blueberry-derived flavonoids may also act to enhance the efficiency of spatial memory indirectly by acting on the dentate gyrus (DG), the hippocampal subregion most sensitive to the effects of aging $(56,425)$. Blueberry supplementation has been shown to significantly increase the proliferation of precursor cells in the DG of aged rats (65). This link between DG neurogenesis, cognitive performance, and aging is well documented (120, 246, 260, 420, 441), and may represent another mechanism by which fruits rich in (poly)phenols may improve memory by acting on the hippocampus. In addition to those with berries, animal studies with tea (70) and pomegranate juice (180) or pure flavonols such as quercetin, rutin (363), or fisetin (290) have provided further evidence that dietary flavonoids are beneficial in reversing the course of neuronal and behavioral aging. There are also lines of evidence suggesting that these effects may not be restricted to memory and learning, but may also exert positive changes in psychomotor activity in older animals $(229,422)$.

\section{C. (Poly)phenols and cancer}

A number of epidemiological studies have investigated the relationship between the intake of fruits and vegetables and cancer incidence, with many indicating a positive correlation $(29,137,140,157,510)$ and others suggesting no association $(44,272,273)$. While a number of in vitro experiments have suggested that (poly)phenols may influence carcinogenesis and tumor development $(61,126,306,356,470)$, very few clinical trials have been conducted regarding the role of (poly)phenols in cancer prevention, incidence, or mortality. Two trials have investigated the effects of green tea extracts on oral cancer $(278,463)$. The first suggested that they may reduce the oral mucosa leukoplakia size, a precursor lesion for oral cancer, following a 6 months of intake of $3 \mathrm{~g} /$ day of a mixed tea extract in capsules (278), whereas the more recent study failed to observe similar reductions in oral premalignant lesions or histology after a 12-week green tea extract supplementation, or any changes in cancer incidence 27 months after trial completion (463). Furthemore, tea (poly)phenol intake (100-600 mg/day) did not decrease serum pepsinogen levels, an indicator of increased risk of stomach cancer, after a year of intake (177), or alter abnormal cell proliferation or lesion formation in patients with esophageal precancerous lesions ( $5 \mathrm{mg}$ of decaffeinated green tea extract/day for 1 year) (483). Data have been more promising in men with high-grade prostatic intraepithelial neoplasia, a precursor of prostate cancer, where daily consumption of green tea catechin capsules ( $200 \mathrm{mg}$ of flavan-3-ols/day) for 1 year resulted in only one tumor being diagnosed among the 30 subjects in the green tea group versus 9 cancers diagnosed in the similarly sized control group (30).

It has been suggested that soy isoflavones may have protective effects against breast cancer $(460,501)$. However, a meta-analysis bringing together eight RCTs investigating the effect of isoflavone consumption $(40-120 \mathrm{mg} /$ day, $0.5-3$ years) on the breast density (a biomarker of breast cancer risk) reported no significant effects, although it appeared that there might be a small increase in the breast density of premenopausal women (212). The clinical relevance of these findings was questioned by the authors and needs to be further investigated.

Other clinical studies have focused on how (poly)phenol interventions influence DNA oxidation/damage, which has been correlated with cancer risk (34). For example, a variety of (poly)phenol-rich foods have been found to protect against lymphocyte DNA damage, including anthocyanin-rich juice (700 $\mathrm{ml}$ of a red mixed berry juice/day; 4 weeks) (487), bloodorange juice $(300 \mathrm{ml} /$ day, 3 weeks) (381), purple potato (150 g/day, 6 weeks) (242), and kiwi fruit (500 ml juice, 1 day) (82). Other trials have reported a decrease in urinary levels of 8-hydroxydeoxyguanosine, which is a biomarker of oxidative DNA damage, following the regular consumption of green tea 
(poly)phenols [ 4 cups/day, $146 \mathrm{mg}$ total (poly)phenols/cup, 4 months; 500/1000 mg (poly)phenols/day, 3 months] (176, 287). Interventions aimed at increasing overall fruit and vegetable intake have also been suggested to induce significant reductions in oxidative DNA damage $(230,231,446,455)$, although such findings are disputed $(52,313)$. Indeed, no influence on DNA oxidation is reported for a dealcoholized, procyanidin-rich wine $(500 \mathrm{ml} /$ day, $7 \mathrm{mg}$ procyanidins $/ \mathrm{kg}$ body weight, 4 weeks) (152), cranberry juice $(750 \mathrm{ml} /$ day, 2 weeks) (127), a mixture of grapes and berries (200 g/day, 2 weeks) (63), or after blackcurrant juice consumption $(666 \mathrm{ml} /$ day, $397 \mathrm{mg}$ anthocyanins/day, 3 weeks) (312). The variability observed here is likely to be influenced by the intervention, its duration, the health status of the study population, and differences in methodology.

A summary of the potential effects of dietary (poly)phenols in humans is illustrated in Figure 41.

\section{Conclusions}

A number of small-scale, human intervention studies have shed light on the beneficial effects of various (poly)phenolrich foods on CVD risk factors. However, many of these studies are limited in that they lack proper control or do not fully characterize their intervention, in particular the (poly)phenol content. For example, if a study uses nothing or water as a control, such a control may well lack the various macro- and micronutrients that are part of the (poly)phenol- rich test intervention, and which may themselves exert efficacy (e.g., vitamin $\mathrm{C} / \mathrm{E}$, folate, and Se). Others do not report the (poly)phenol content and the compositional analysis of the test food/drink, creating significant problems when comparing different studies with the same food/beverage. Smaller issues, such as a failure to blind the study and a failure to record compliance and absorption/metabolism data, also limit the interpretation of the existing data. At present, uncertainty remains regarding which specific (poly)phenols are directly responsible for beneficial actions in vivo, as many of these foods/beverages contain a diversity of (poly)phenols classes (see Section II).

At present, the strongest evidence for the efficacy of (poly)phenols against chronic disease exists for flavan-3-olrich foods, in particular cocoa in respect to CVD risk. Many flavan-3-ol studies have used adequate controls in doubleblind randomized studies, with well-characterized intervention foods/drinks. Additionally, they have strived to assess flavan-3-ol absorption and metabolism, a factor critical in building potential cause-and-effect relationships. Flavan-3-ol studies have been further strengthened by interventions, indicating that pure flavan-3-ols mimic the vascular effects exerted by flavan-3-ol-rich foods. As such, there is good evidence to suggest that the acute and/or regular consumption of flavan-3-ol rich cocoa or tea can have a positive effect on the vascular system, mainly through their effects on endothelial function, NO bioavailability, and BP. However, the long-term effects of flavan-3-ol interventions are presently
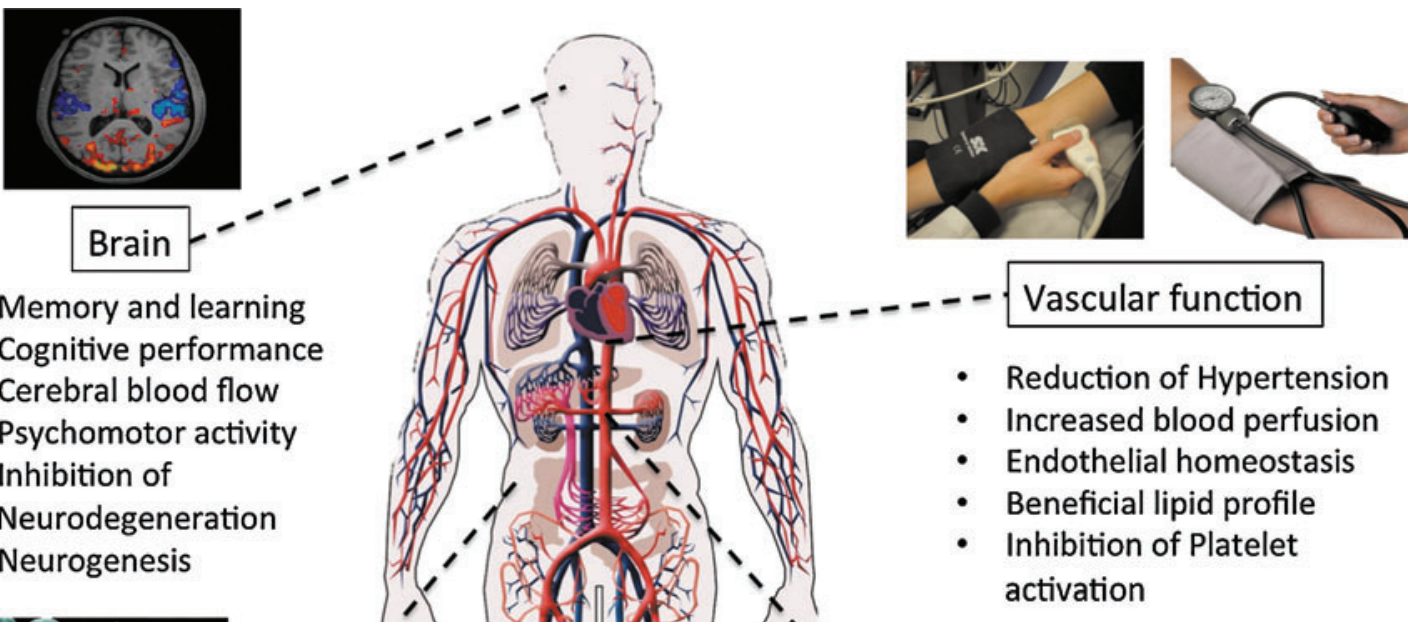

- Memory and learning

- Cognitive performance

- Cerebral blood flow

- Psychomotor activity

- Inhibition of Neurodegeneration

- Neurogenesis

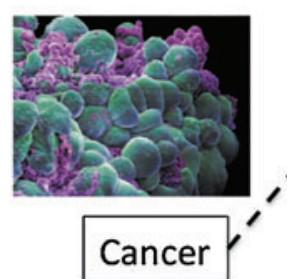

- Inhibition of tumor development

- Detoxification of cancer precursors

- Cancer cell apoptosis

- Inhibition of DNA oxidation

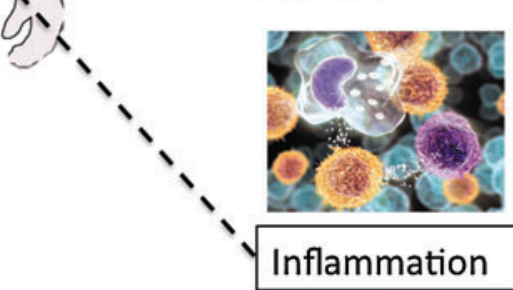

- Inhibition of cytokine/chemokine production mediators

- Reduced of adhesion molecule expression

- Inhibition of Neuroinflammation

FIG. 41. A summary of the potential health benefits of dietary (poly)phenols. (To see this illustration in color, the reader is referred to the web version of this article at www.liebertpub.com/ars.) 
unclear, and there are issues to resolve with respect to the efficacy of tea and caffeine.

Presently, the evidence for anthocyanins, flavonols and flavanones, stilbenes, hydroxycinnamates, and phenolic acids, based on studies with berries, pomegranate, red wine or citrus fruits, and coffee, is inconclusive, mainly due to the insufficiencies in the study design mentioned above. The diversity of (poly)phenols in berries and wine also makes it more difficult to assess putative health effects of individual (poly)phenols. Future studies will need to utilize other pure (poly)phenols in well-designed RCTs, before conclusions can be drawn regarding their specific biological function. Recent studies with flavanone-rich orange juice in parallel with pure flavanones are encouraging, in particular the positive effects exerted by pure flavanones on endothelial function and BP. Data with nuts are undoubtedly complicated by the presence of unsaturated fats, which are also known to be protective against various vascular risk factors. Future work with nuts will need to isolate specific (poly)phenol fractions for intervention to shed further light on the efficacy of nut (poly)phenols.

While there appears to be vast literature regarding the potential of (poly)phenols to improve cardiovascular health, at present, there is insufficient evidence to correlate individual (poly)phenols, with the exception of flavan-3-ol monomers and flavanones, with benefits against CVD risk, particularly with regard to long-term ingestion and risk. As such, additional long-term RCTs are required that utilize better-defined interventions, which are properly controlled and blinded, and that assess physiological endpoints linked to disease. Such studies should include detailed compositional analysis of the (poly) phenol-rich foods and control food/beverage carried out using validated analytical methods. If possible, future studies should include a pure (poly)phenol arm and controls designed accordingly to investigate the efficacy of specific (poly)phenols on functional outcomes. If such control arms are not possible (i.e., lack of a food grade quality, pure (poly)phenol in sufficient quantity), it is crucial that studies attempt to assess the absorption and metabolism of the (poly)phenols from the food/ beverage that functional outcomes can be directly correlated to the presence of (poly)phenols/metabolites in the circulation. Ideally, what now required are long-term, large population RCT studies with healthy or at-risk individuals and with clinically relevant endpoints. Such studies are likely to be expensive, will require collaboration between academia and industry, and will run over a sufficiently long time frame (e.g., 3-12 months) to meet health claim criteria. Ultimately, (poly)phenols also need to be tested in the context of other unavoidable CVD risk factors, such as age, sex, and/or ethnicity. Inclusions of these are likely to increase the study population size considerably, and thus such studies will require cooperation between multiple centers, each running a cohort with the same dietary intervention/control. The outcomes of such future studies, if positive, may ultimately be used to make specific dietary recommendations regarding the efficacy of (poly)phenols in preventing the CVD risk.

Currently, there are also not enough data to clearly associate (poly)phenol consumption to improvements in neurological health. While there is extensive animal evidence for the actions of flavan-3-ols (from cocoa, wine, grape, etc.) and anthocyanins (from blueberry, grape, raspberry, etc.) on memory and learning, this still needs to be translated into humans before firm efficacy conclusions can be made. In such studies, inter- vention periods may need to be very long (12-24 months) before significant benefits emerge. There are limited data in support of a causal relationship between the consumption of flavonoids and behavioral outcomes. To make such relationships, future intervention studies will be required to utilize better-characterized intervention materials, more appropriate controls, and more rigorous clinical outcomes, such as welldesigned human cognitive testing along with hemodynamic and structural changes measurable using MRI and fMRI techniques. Such hemodynamic changes may be further compared to changes in the gray matter density and to biomarkers of neural stem and progenitor cells using proton nuclear magnetic resonance spectroscopy. Such an approach will be essential to provide links between flavonoid intake and brain function in a mechanistic, dynamic, and quantitative way.

Another mechanism by which (poly)phenols may influence brain disease is through the modification of a number of risk factors common to $\mathrm{AD}$, vascular dementia, hypertension, and CVD $(26,48)$. Modulation of these classical cardiovascular risk factors by flavonoids, as detailed in the previous section, may result in reductions in both typical and atypical cognitive function $(43,50)$. The ability of flavonoids to exert peripheral vascular changes $(30,66,78,83,86,97,98,147,226)$ may facilitate more efficient cerebral blood flow (CBF), something that is known to deteriorate with age, to be vital for optimal brain function, and to be decreased in patients with dementia $(163,201,395,485)$. In this respect, flavan-3-ol-rich cocoa has also been shown to exert a positive effect on CBF through the middle cerebral artery in humans $(55,74,80,113)$. In support of these findings, arterial spin-labeling sequence magnetic resonance imaging also indicates that flavan-3-ols increase CBF up to a maximum of $2 \mathrm{~h}$ after ingestion (142).

In relation to dementia, there is a lack of human clinical evidence to date to make solid conclusions regarding function in patient populations, although the consumption of (poly)phenol-rich vegetables, fruit juices, and red wine has been reported to delay the onset of the disease $(13,50)$. However, the vast majority of data in support of their effects against dementia derive from animal studies that have been focused to a greater degree on the mechanism of action rather than functional outcomes. Further studies are required before conclusions may be drawn. As intervention times will have to be long, careful consideration needs will have to be afforded to whether supplementation with individual flavonoids, flavonoid combinations, or simply clearer dietary recommendations on flavonoid intake (including a potentially recommended daily intake level) represents the best initial approach for the rapid translation of these findings into effective interventions for individuals affected by dementia.

Evidence relating to the anticancer effects of (poly)phenols is limited. The majority of available clinical evidence has been with green tea/(poly)phenols in populations at a high risk of cancer development, with results proving inconclusive. In particular, there are some promising data indicating that regular consumption of green tea may retard oral and prostate cancer development. With regard to oral cancer, the efficacy may be apparent due to the direct interaction between (poly)phenols and cancer cells in the mouth, and suggest that similar effects against other GIT cancers (esophagus, stomach, small, and large intestine) may be possible. Future studies need to be designed in collaboration with cancer biologists and clinicians to test this hypothesis. 
While a few of studies suggest that (poly)phenol-rich foods prevent lymphocyte DNA damage, no direct link with a decrease in cancer risk can be established from those studies. Undoubtedly, the oxidation of DNA may contribute to mutation rates and cancer risk; however, it is unlikely that such a reduction in lymphocyte DNA damage would impact on cancer development in sites outside the GIT, for example, breast, colon, and lung. As discussed earlier, more wellcontrolled, long-term intervention studies are needed, in particular those that measure tumor size, cancer incidence, and even cancer mortality as endpoints. Such endpoints are needed in the absence of suitable biomarkers of cancer risk, and should be measured routinely in healthy individuals or cancer patients. Until such studies have been conducted, it will be difficult to fully assess the impact of (poly)phenol-rich foods on cancer development and long-term risk.

Finally, a number of studies have been recently appearing in the literature overcoming previous limitations in the approaches used to unravel the putative mechanisms of action behind the protective effects of (poly)phenols. Identifying metabolites and catabolites and testing them in cell-based experimental models at physiological concentrations are the only ways to fully understand if the in vivo observations actually have molecular basis. While most of the published articles on (poly)phenol bioactivity still describe the effects of molecules that are only present in planta, detailed and elegant studies applying human and microbial metabolites have established reasonable mechanisms through which some candidates in this immense class of plant secondary metabolites may exert their beneficial actions. In this context, it is apparent that they do not act simply as antioxidants in vivo, and their diverse effects are, in most instances, based on more complex and specific modes of action, as discussed in Section V. Along with medium long-term RCTs, new and more refined molecular approaches will be welcome to finally and fully understand how these molecules, which have been relegated to the promising category for far too long, interact with human physiological and pathological processes. As yet, it is too premature to consider the potential use of (poly)phenolic compounds as therapeutic agents.

\section{Acknowledgment}

Supported by "Progetto AGER," grant n. 2011-0283.

\section{References}

1. Adams LS, Zhang Y, Seeram NP, Heber D, and Chen S. Pomegranate ellagitannin-derived compounds exhibit antiproliferative and antiaromatase activity in breast cancer cells in vitro. Cancer Prev Res 3: 108-113, 2010.

2. Adlercreutz H. Lignans and human health. Crit Rev Clin Lab Sci 44: 483-525, 2007.

3. Agewall S, Wright S, Doughty RN, Whalley GA, Duxbury $\mathrm{M}$, and Sharpe N. Does a glass of red wine improve endothelial function? Eur Heart J 21: 74-78, 2000.

4. Aggarwal BB and Sung B. The relationship between inflammation and cancer is analogous to that between fuel and fire. Oncology 25: 414-418, 2011.

5. Al-Shalmani S, Suri S, Hughes DA, Kroon PA, Needs PW, Taylor MA, Tribolo S, and Wilson VG. Quercetin and its principal metabolites, but not myricetin, oppose lipopoly- saccharide-induced hyporesponsiveness of the porcine isolated coronary artery. Br J Pharmacol 162: 1485-1497, 2011.

6. Albers AR, Varghese S, Vitseva O, Vita JA, and Freedman JE. The antiinflammatory effects of purple grape juice consumption in subjects with stable coronary artery disease. Arterioscler Thromb Vasc Biol 24: e179-e180, 2004.

7. Alexopoulos N, Vlachopoulos C, Aznaouridis K, Baou K, Vasiliadou C, Pietri P, Xaplanteris P, Stefanadi E, and Stefanadis $C$. The acute effect of green tea consumption on endothelial function in healthy individuals. Eur J Cardiovasc Prev Rehabil 15: 300-305, 2008.

8. Almeida L, Vaz-da-Silva M, Falcão A, Soares E, Costa R, Loureiro AI, Fernandes-Lopes C, Rocha JF, Nunes T, Wright L, and Soares-da-Silva P. Pharmacokinetic and safety profile of trans-resveratrol in a rising multiple-dose study in healthy volunteers. Mol Nutr Food Res 53: Suppl 1, S7-S15, 2009.

9. Andres-Lacueva C, Shukitt-Hale B, Galli RL, Jauregui O, Lamuela-Raventos RM, and Joseph JA. Anthocyanins in aged blueberry-fed rats are found centrally and may enhance memory. Nutr Neurosci 8: 111-120, 2005.

10. Appeldoom MM, Vincken J-P, Aura A-M, Hollman PC, and Gruppen H. Procyanidin dimers are metabolized by human microbiota with 2-(3,4-dihydroxyphenyl)acetic acid and 5-(3,4-dihydroxyphenyl)- $\gamma$-valerolactone as the major metabolites. J Agric Food Chem 57: 1084-1092, 2009.

11. Ardalan MR, Tarzamni MK, Shoja MM, Tubbs RS, RahimiArdabili B, Ghabili K, and Khosroshahi HT. Black tea improves endothelial function in renal transplant recipients. Transplant Proc 39: 1139-1142, 2007.

12. Aron PM and Kennedy JA. Flavan-3-ols: nature, occurrence and biological activity. Mol Nutr Food Res 52: 79-104, 2008.

13. Arts IC and Hollman PC. Polyphenols and disease risk in epidemiologic studies. Am J Clin Nutr 81: 317S-325S, 2005.

14. Auger C, Hara Y, and Crozier A. Bioavailability of polyphenon E flavan-3-ols in humans with an ileostomy. J Nutr 138: 1535S-1542S, 2008.

15. Aura A-M. Colon-derived microbial metabolites of dietary phenolic compounds. In: Flavonoids and Related Compounds: Bioavailability and Function, edited by Spencer JPE and Crozier A. Oxidative Stress and Disease, Vol. 30, edited by Packer L and Cadenas H. Boca Raton, FL: CRC Press, 2012, pp. 201-231.

16. Aura A-M, Oikarinen S, Mutanen M, Heinonen SM, Adlercreutz HC, Virtanen $\mathrm{H}$, and Poutanen KS. Suitability of a batch in vitro fermentation model using human faecal microbiota for prediction of conversion of flaxseed lignans to enterolactone with reference to an in vivo rat model. Eur J Nutr 45: 45-51, 2006.

17. Aviram M, Dornfeld L, Kaplan M, Coleman R, Gaitini D, Nitecki S, Hofman A, Rosenblat M, Volkova N, Presser D, Attias J, Hayek T, and Fuhrman B. Pomegranate juice flavonoids inhibit low-density lipoprotein oxidation and cardiovascular diseases: studies in atherosclerotic mice and in humans. Drugs Exp Clin Res 28: 49-62, 2002.

18. Aviram M, Rosenblat M, Gaitini D, Nitecki S, Hoffman A, Dornfeld L, Volkova N, Presser D, Attias J, Liker H, and Hayek T. Pomegranate juice consumption for 3 years by patients with carotid artery stenosis reduces common carotid intima-media thickness, blood pressure and LDL oxidation. Clin Nutr 23: 423-433, 2004.

19. Aviram M, Volkova N, Coleman R, Dreher M, Reddy MK, Ferreira D, and Rosenblat M. Pomegranate phenolics from 
the peels, arils, and flowers are antiatherogenic: studies in vivo in atherosclerotic apolipoprotein e-deficient (E 0) mice and in vitro in cultured macrophages and lipoproteins. J Agric Food Chem 56: 1148-1157, 2008.

20. Baba S, Osakabe N, Yasuda A, Natsume M, Takizawa T, Nakamura T, and Terao J. Bioavailability of (-)-epicatechin upon intake of chocolate and cocoa in human volunteers. Free Radic Res 33: 635-641, 2000.

21. Bahorun T, Luximon-Ramma A, Neergheen-Bhujun VS, Gunness TK, Googoolye K, Auger C, Crozier A, and Aruoma OI. The effect of black tea on risk factors of cardiovascular disease in a normal population. Prev Med 54: S98S102, 2012.

22. Balzer J, Rassaf T, Heiss C, Kleinbongard P, Lauer T, Merx M, Heussen N, Gross HB, Keen CL, Schroeter H, and Kelm M. Sustained benefits in vascular function through flavanol-containing cocoa in medicated diabetic patients a double-masked, randomized, controlled trial. J Am Coll Cardiol 51: 2141-2149, 2008.

23. Banel DK and Hu FB. Effects of walnut consumption on blood lipids and other cardiovascular risk factors: a metaanalysis and systematic review. Am J Clin Nutr 90: 56-63, 2009.

24. Barberger-Gateau P, Raffaitin C, Letenneur L, Berr C, Tzourio C, Dartigues JF, and Alperovitch A. Dietary patterns and risk of dementia: the Three-City cohort study. Neurology 69: 1921-1930, 2007.

25. Barron D, Smarrito-Menozzi C, Fumeaux R, and Viton F. Synthesis of dietary phenolic metabolites and isotopicallylabeled dietary phenolics. In: Bioavailability and Function of Flavonoids, edited by Spencer JPE and Crozier A. Oxidative Stress and Disease, Vol. 30, edited by Packer L and Cadenas H. Boca Raton, FL: CRC Press, 2012, pp. 233-280.

26. Barros D, Amaral OB, Izquierdo I, Geracitano L, do Carmo Bassols Raseira M, Henriques AT, and Ramirez MR. Behavioral and genoprotective effects of Vaccinium berries intake in mice. Pharmacol Biochem Behav 84: 229-234, 2006.

27. Basu A, Du M, Leyva MJ, Sanchez K, Betts NM, Wu M, Aston CE, and Lyons TJ. Blueberries decrease cardiovascular risk factors in obese men and women with metabolic syndrome. J Nutr 140: 1582-1587, 2010.

28. Basu A, Sanchez K, Leyva MJ, Wu M, Betts NM, Aston CE, and Lyons TJ. Green tea supplementation affects body weight, lipids, and lipid peroxidation in obese subjects with metabolic syndrome. J Am Coll Nutr 29: 31-40, 2010.

29. Benetou V, Orfanos P, Lagiou P, Trichopoulos D, Boffetta $\mathrm{P}$, and Trichopoulou A. Vegetables and fruits in relation to cancer risk: evidence from the Greek EPIC cohort study. Cancer Epidemiol Biomarkers Prev 17: 387-392, 2008.

30. Bettuzzi S, Brausi M, Rizzi F, Castagnetti G, Peracchia G, and Corti A. Chemoprevention of human prostate cancer by oral administration of green tea catechins in volunteers with high-grade prostate intraepithelial neoplasia: a preliminary report from a one-year proof-of-principle study. Cancer Res 66: 1234-1240, 2006.

31. Bialonska D, Kasimsetty SG, Khan SI, and Ferreira D. Urolithins, intestinal microbial metabolites of pomegranate ellagitannins, exhibit potent antioxidant activity in a cell-based assay. J Agric Food Chem 57: 10181-10186, 2009.

32. Bingham SA, Vorster H, Jerling JC, Magee E, Mulligan A, Runswick SA, and Cummings JH. Effect of black tea drinking on blood lipids, blood pressure and aspects of bowel habit. Br J Nutr 78: 41-55, 1997.
33. Birge SJ. Is there a role for estrogen replacement therapy in the prevention and treatment of dementia? J Am Geriatr Soc 44: 865-870, 1996.

34. Bjelland S and Seeberg E. Mutagenicity, toxicity and repair of DNA base damage induced by oxidation. Mutat Res 531: 37-80, 2003.

35. Boban M, Modun D, Music I, Vukovic J, Brizic I, Salamunic I, Obad A, Palada I, and Dujic Z. Red wine induced modulation of vascular function: separating the role of polyphenols, ethanol, and urates. J Cardiovasc Pharm 47: 695701, 2006.

36. Boesch-Saadatmandi C, Loboda A, Wagner AE, Stachurska A, Jozkowicz A, Dulak J, Döring F, Wolffram S, and Rimbach G. Effect of quercetin and its metabolites isorhamnetin and quercetin-3-glucuronide on inflammatory gene expression: role of miR-155. J Nutr Biochem 22: 293299, 2011.

37. Bolling BW, Chen CY, McKay DL, and Blumberg JB. Tree nut phytochemicals: composition, antioxidant capacity, bioactivity, impact factors. A systematic review of almonds, Brazils, cashews, hazelnuts, macadamias, pecans, pine nuts, pistachios and walnuts. Nutr Res Rev 24: 1-32, 2011.

38. Bonetti A, Marotti I, and Dinelli G. Urinary excretion of kaempferol from common beans (Phaseolus vulgaris L.) in humans. Int J Food Sci 58: 261-269, 2007.

39. Boocock DJ, Faust GE, Patel KR, Schinas AM, Brown VA, Ducharme MP, Booth TD, Crowell JA, Perloff M, Gescher AJ, Steward WP, and Brenner DE. Phase I dose escalation pharmacokinetic study in healthy volunteers of resveratrol, a potential cancer chemopreventive agent. Cancer Epidemiol Biomarkers Prev 16: 1246-1252, 2007.

40. Boocock DJ, Patel KR, Faust GE, Normolle DP, Marczylo $\mathrm{TH}$, Crowell JA, Brenner DE, Booth TD, Gescher A, and Steward WP. Quantitation of trans-resveratrol and detection of its metabolites in human plasma and urine by high performance liquid chromatography. Chromatogr B 848: 182-187, 2007.

41. Borges G, Degeneve A, Mullen W, and Crozier A. Identification of flavonoid and phenolic antioxidants in blackcurrants, blueberries, raspberries, redcurrants and cranberries. J Agric Food Chem 58: 3901-3909, 2010.

42. Borges G, Mullen W, Mullan A, Lean MEJ, Roberts SA, and Crozier A. Bioavailability of multiple components following acute ingestion of a polyphenol-rich drink. Mol Nutr Food Res 5: S268-S277, 2010.

43. Borges G, Roowi S, Rouanet J-M, Duthie GG, Lean MEJ, and Crozier A. The bioavailability of raspberry anthocyanins and ellagitannins in rats. Mol Nutr Food Res 51: 714725, 2007.

44. Botterweck AA, van den Brandt PA, and Goldbohm RA. A prospective cohort study on vegetable and fruit consumption and stomach cancer risk in The Netherlands. Am J Epidemiol 148: 842-853, 1998.

45. Braune A, Engst W, and Blaut M. Degradation of neohesperidin dihydrochalcone by human intestinal bacteria. $J$ Agric Food Chem 53: 1782-1790, 2005.

46. Bredsdorff L, Nielson ILF, Rasmussen SE, Cornett C, Barron D, Bouisset F, Offord E, and Williamson G. Absorption, conjugation and excretion of the flavanones, naringenin and hesperetin from $\alpha$-rhamnosidase - treated orange juice in human subjects. Br J Nutr 103: 1602-1609, 2010.

47. Brenn T. The Tromso heart study: alcoholic beverages and coronary risk factors. J Epidemiol Community Health 40: 249256, 1986. 
48. Breteler MM. Vascular risk factors for Alzheimer's disease: an epidemiologic perspective. Neurobiol Aging 21: 153-160, 2000.

49. Brett GM, Hollands W, Needs PW, Teucher B, Dainty JR, Davis BD, Brodbelt JS, and Kroon PA. Absorption, metabolism and excretion of flavanones from singles portion of orange fruit and juice and effects of anthropometric variables and contraceptive pill use on flavanones excretion. Br J Nutr 101: 1-12, 2008.

50. Bridges JW, French MR, Smith RL, and Williams RT. The fate of benzoic acid in various species. Biochem J 118: 47-51, 1970.

51. Brien SE, Ronksley PE, Turner BJ, Mukamal KJ, and Ghali WA. Effect of alcohol consumption on biological markers associated with risk of coronary heart disease: systematic review and meta-analysis of interventional studies. BMJ 342: d636, 2011.

52. Briviba K, Bub A, Moseneder J, Schwerdtle T, Hartwig A, Kulling S, and Watzl B. No differences in DNA damage and antioxidant capacity between intervention groups of healthy, nonsmoking men receiving 2, 5, or 8 servings/day of vegetables and fruit. Nutr Cancer 60: 164-170, 2008.

53. Brown AL, Lane J, Holyoak C, Nicol B, Mayes AE, and Dadd $\mathrm{T}$. Health effects of green tea catechins in overweight and obese men: a randomised controlled cross-over trial. $\mathrm{Br}$ J Nutr 106: 1880-1889, 2011.

54. Buijsse B, Weikert C, Drogan D, Bergmann M, and Boeing $\mathrm{H}$. Chocolate consumption in relation to blood pressure and risk of cardiovascular disease in German adults. Eur Heart J 31: 1616-1623, 2010.

55. Bulpitt CJ, Shipley MJ, and Semmence A. The contribution of a moderate intake of alcohol to the presence of hypertension. J Hypertens 5: 85-91, 1987.

56. Burke SN and Barnes CA. Neural plasticity in the ageing brain. Nat Rev Neurosci 7: 30-40, 2006.

57. Burkon A and Somoza V. Quantification of free and protein bound trans-resveratrol metabolites and identification of trans-resveratrol-C/O-conjugated diglucuronides-two novel resveratrol metabolites in human plasma. Mol Nutr Food Res 52: 549-557, 2008.

58. Buscemi S, Verga S, Batsis JA, Donatelli M, Tranchina MR, Belmonte S, Mattina A, Re A, and Cerasola G. Acute effects of coffee on endothelial function in healthy subjects. Eur J Clin Nutr 64: 483-489, 2010.

59. Buscemi S, Verga S, Batsis JA, Tranchina MR, Belmonte S, Mattina A, Re A, Rizzo R, and Cerasola G. Dose-dependent effects of decaffeinated coffee on endothelial function in healthy subjects. Eur J Clin Nutr 63: 1200-1205, 2009.

60. Calani L, Del Rio D, Luisa Callegari M, Morelli L, and Brighenti F. Updated bioavailability and $48 \mathrm{~h}$ excretion profile of flavan-3-ols from green tea in humans. Int J Food Sci Nutr 63: 513-521, 2012.

61. Calomme M, Pieters L, Vlietinck A, and Vanden Berghe D. Inhibition of bacterial mutagenesis by citrus flavonoids. Planta Med 62: 222-226, 1996.

62. Carkeet C, Clevidence BA, and Novotny JA. Anthocyanin excretion by humans increases linearly with increasing strawberry dose. J Nutr 138: 897-902, 2008.

63. Carmen Ramirez-Tortosa M, Garcia-Alonso J, Luisa Vidal-Guevara M, Quiles JL, Jesus Periago M, Linde J, Dolores Mesa M, Ros G, Abellan P, and Gil A. Oxidative stress status in an institutionalised elderly group after the intake of a phenolic-rich dessert. Br J Nutr 91: 943950, 2004.
64. Carreau C, Flouriot G, Bennetau-Pelissero C, and Potier M. Enterodiol and enterolactone, two major diet-derived polyphenol metabolites have different impact on ER $\alpha$ transcriptional activation in human breast cancer cells. $J$ Steroid Biochem Mol Biol 110: 176-185, 2008.

65. Casadesus G, Shukitt-Hale B, Stellwagen HM, Zhu X, Lee HG, Smith MA, and Joseph JA. Modulation of hippocampal plasticity and cognitive behavior by short-term blueberry supplementation in aged rats. Nutr Neurosci 7: 309316, 2004.

66. Casas-Agustench P, Lopez-Uriarte P, Ros E, Bullo M, and Salas-Salvado J. Nuts, hypertension and endothelial function. Nutr Metab Cardiovasc Dis 21 Suppl 1: S21-S33, 2011.

67. Casini ML, Marelli G, Papaleo E, Ferrari A, D’Ambrosio F, and Unfer V. Psychological assessment of the effects of treatment with phytoestrogens on postmenopausal women: a randomized, double-blind, crossover, placebo-controlled study. Fertil Steril 85: 972-978, 2006.

68. Cerdá B, Espín JC, Parra S, Martínez P, and Tomás-Barberán FA. The potent in vitro antioxidant ellagitannins from pomegranate juice are metabolised into bioavailable but poor antioxidant hydroxy-6H-dibenzopyran-6-one derivatives by the colonic microflora of healthy humans. Eur J Nutr 43: 205-220, 2004.

69. Cerdá B, Tomás-Barberán FA, and Espín JC. Metabolism of antioxidant and chemopreventive ellagitannins from strawberries, raspberries, walnuts, and oak-aged wine in humans: identification of biomarkers and individual variability. J Agric Food Chem 53: 227-235, 2005.

70. Chan YC, Hosoda K, Tsai CJ, Yamamoto S, and Wang MF. Favorable effects of tea on reducing the cognitive deficits and brain morphological changes in senescence-accelerated mice. J Nutr Sci Vitaminol 52: 266-273, 2006.

71. Chao CL, Hou YC, Chao PD, Weng CS, and Ho FM. The antioxidant effects of quercetin metabolites on the prevention of high glucose-induced apoptosis of human umbilical vein endothelial cells. Br J Nutr 101: 1165-1170, 2009.

72. Chaves AA, Joshi MS, Coyle CM, Brady JE, Dech SJ, Schanbacher BL, Baliga R, Basuray A, and Bauer JA. Vasoprotective endothelial effects of a standardized grape product in humans. Vascul Pharmacol 50: 20-26, 2009.

73. Chen LH, Fang J, Li H, Demark-Wahnefried W, and Lin X. Enterolactone induces apoptosis in human prostate carcinoma LNCaP cells via a mitochondrial-mediated, caspasedependent pathway. Mol Cancer Ther 6: 2581-2590, 2007.

74. Cheng C, Wang X, Weakley SM, Kougias P, Lin PH, Yao Q, and Chen $\mathrm{C}$. The soybean isoflavonoid equol blocks ritonavir-induced endothelial dysfunction in porcine pulmonary arteries and human pulmonary artery endothelial cells. J Nutr 140: 12-17, 2010.

75. Chow HH, Cai Y, Alberts DS, Hakim I, Dorr R, Shahi F, Crowell JA, Yang CS, and Hara Y. Phase I pharmacokinetic study of tea polyphenols following single-dose administration of epigallocatechin gallate and Polyphenon E. Cancer Epidemiol Biomarkers Prev 10: 53-58, 2001.

76. Chu LM, Lassaletta AD, Robich MP, and Sellke FW. Resveratrol in the prevention and treatment of coronary artery disease. Curr Atheroscl Rep 13: 439-446, 2011.

77. Clavel T, Henderson G, Alpert C-A, Philippe C, RigottierGois L, Doré J, and Blaut M. Intestinal bacterial communities that produce active estrogen-like compounds enterodiol and enterolactone in humans. Appl Environ Microbiol 71: 6077-6085, 2005. 
78. Clifford MN. Anthocyanins-nature, occurrence and dietary burden. J Sci Food Agric 80: 1063-1072, 2000.

79. Clifford MN, Copeland EL, Bloxsidge JP, and Mitchell LA. Hippuric acid as a major excretion product associated with black tea consumption. Xenobiotica 30: 317-326, 2000.

80. Clifford MN and Crozier A. Phytochemicals in teas and tisanes and their bioavailability. In: Teas, Cocoa and Coffee: Plant Secondary Metabolites and Health, edited by Crozier A, Ashihara $\mathrm{H}$, and Tomás-Barbéran F. Oxford: Blackwell Publishing, 2011, pp. 45-98.

81. Coldham NG, Darby C, Hows M, King LJ, Zhang AQ, and Saurer MJ. Comparative metabolism of genistein in human and rat gut microflora: detection and identification of the end-products of metabolism. Xenobiotica 32: 45-62, 2002.

82. Collins BH, Horska A, Hotten PM, Riddoch C, and Collins AR. Kiwifruit protects against oxidative DNA damage in human cells and in vitro. Nutr Cancer 39: 148-153, 2001.

83. Commenges D, Scotet V, Renaud S, Jacqmin-Gadda $H$, Barberger-Gateau $\mathrm{P}$, and Dartigues JF. Intake of flavonoids and risk of dementia. Eur J Epidemiol 16: 357-363, 2000.

84. Corder R. Red wine, chocolate and vascular health: developing the evidence base. Heart 94: 821-823, 2008.

85. Corder R, Crozier A, and Kroon PA. Drinking your health? It's too early to say. Nature 426: 119, 2003.

86. Cordova AC, Jackson LS, Berke-Schlessel DW, and Sumpio $\mathrm{BE}$. The cardiovascular protective effect of red wine. J Am Coll Surg 200: 428-439, 2005.

87. Corsini E, Dell'Agli M, Facchi A, De Fabiani E, Lucchi L, Boraso MS, Marinovich M, and Galli CL. Enterodiol and enterolactone modulate the immune response by acting on nuclear factor $-\kappa \mathrm{B}(\mathrm{NF}-\kappa \mathrm{B})$ signaling. J Agric Food Chem 58: 6678-6684, 2010.

88. Coward L, Smith M, Kirk M, and Barnes S. Chemical modification of isoflavones in soyfoods during cooking and processing. Am J Clin Nutr 68: 1486S-1491S, 1998.

89. Crews WD, Jr., Harrison DW, and Wright JW. A doubleblind, placebo-controlled, randomized trial of the effects of dark chocolate and cocoa on variables associated with neuropsychological functioning and cardiovascular health: clinical findings from a sample of healthy, cognitively intact older adults. Am J Clin Nutr 87: 872-880, 2008.

90. Crowe FL, Roddam AW, Key TJ, Appleby PN, Overvad K, Jakobsen MU, Tjonneland A, Hansen L, Boeing H, Weikert C, Linseisen J, Kaaks R, Trichopoulou A, Misirli G, Lagiou P, Sacerdote C, Pala V, Palli D, Tumino R, Panico S, Buenode-Mesquita HB, Boer J, van Gils $\mathrm{CH}$, Beulens JW, Barricarte A, Rodriguez L, Larranaga N, Sanchez MJ, Tormo MJ, Buckland G, Lund E, Hedblad B, Melander O, Jansson JH, Wennberg P, Wareham NJ, Slimani N, Romieu I, Jenab M, Danesh J, Gallo V, Norat T, and Riboli E. Fruit and vegetable intake and mortality from ischaemic heart disease: results from the European Prospective Investigation into Cancer and Nutrition (EPIC)-Heart study. Eur Heart J 32: 1235-1243, 2011.

91. Crozier A, Ashihara H, and Tomás-Barbéran F (eds.). Teas, Cocoa and Coffee: Plant Secondary Metabolites and Health. Oxford: Blackwell Publishing, 2011.

92. Crozier A, Borges G, and Ryan D. The glass that cheers: phenolic and polyphenolic constituents and the beneficial effects of moderate red wine consumption. Biochemist 32: 49, 2010

93. Crozier A, Jaganath IB, and Clifford MN. Dietary phenolics: chemistry, bioavailability and effects on health. Nat Prod Rep 26: 1001-1043, 2009.
94. Crozier A, McDonald MS, Lean MEJ, and Black C. Quantitative analysis of the flavonoid content of tomatoes, onions, lettuce and celery. J Agric Food Chem 45: 590-595, 1997.

95. Crozier A, Yokota T, Jaganath IB, Marks S, Saltmarsh M, and Clifford MN. Secondary metabolites as dietary components in plant-based foods and beverages. In: Plant Secondary Metabolites: Occurrence, Structure and Role in the Human Diet, edited by Crozier A, Clifford MN, and Ashihara H. Oxford: Blackwell Publishing, 2006, pp. 208-302.

96. Crozier TWM, Stalmach A, Lean MEJ, and Crozier A. Espresso coffees, caffeine and chlorogenic acid intake: potential health implications. Food Funct 3: 30-33, 2012.

97. Cutler GJ, Nettleton JA, Ross JA, Harnack LJ, Jacobs DR, Jr., Scrafford CG, Barraj LM, Mink PJ, and Robien K. Dietary flavonoid intake and risk of cancer in postmenopausal women: the Iowa Women's Health Study. Int J Cancer 123: 664-671, 2008.

98. da Silva EL, Piskula MK, Yamamoto N, Moon JH, and Terao J. Quercetin metabolites inhibit copper ion-induced lipid peroxidation in rat plasma. FEBS Lett 430: 405-408, 1998.

99. Dai Q, Borenstein AR, Wu Y, Jackson JC, and Larson EB. Fruit and vegetable juices and Alzheimer's disease: the Kame Project. Am J Med 119: 751-759, 2006.

100. Dauchet L, Amouyel P, Hercberg S, and Dallongeville J. Fruit and vegetable consumption and risk of coronary heart disease: a meta-analysis of cohort studies. J Nutr 136: 2588 2593, 2006.

101. Davidson MH, Maki KC, Dicklin MR, Feinstein SB, Witchger M, Bell M, McGuire DK, Provost JC, Liker H, and Aviram M. Effects of consumption of pomegranate juice on carotid intima-media thickness in men and women at moderate risk for coronary heart disease. Am J Cardiol 104: 936-942, 2009.

102. Davison K, Coates AM, Buckley JD, and Howe PR. Effect of cocoa flavanols and exercise on cardiometabolic risk factors in overweight and obese subjects. Int J Obes 32: 1289-1296, 2008.

103. Day AJ, Bao Y, Morgan MR, and Williamson G. Conjugation position of quercetin glucuronides and effect on biological activity. Free Radic Biol Med 29: 12341243, 2000.

104. Day AJ, Canada FJ, Diaz JC, Kroon PA, Mclauchlan R, Faulds CB, Plumb GW, Morgan MR, and Williamson G. Dietary flavonoid and isoflavone glycosides are hydrolysed by the lactase site of lactase phloridzin hydrolase. FEBS Lett 468: 166-170, 2000.

105. Day AJ, Mellon F, Barron D, Sarrazin G, Morgan MRA, and Williamson G. Human metabolism of dietary flavonoids: identification of plasma metabolites of quercetin. Free Radic Res 35: 941-952, 2001.

106. Day AJ and Morgan MRA. Methods of polyphenol extraction from biological fluids and tissues. In: Methods in Polyphenol Analysis, edited by Santos-Buelga C and Williamson G. Cambridge: Royal Society of Chemistry, 2003, pp. $17-47$.

107. Daykin CA, van Duynhoven JP, Groenewegen A, Dachtler M, van Amelsvoort JM, and Mulder TP. Nuclear magnetic resonance spectroscopic based studies of the metabolism of black tea polyphenols in humans. J Agric Food Chem 53: 1428-1434, 2005.

108. Del Rio D, Calani L, Cordero C, Salvatore S, Pellegrini N, and Brighenti F. Bioavailability and catabolism of green tea flavan-3-ols in humans. Nutrition 26: 1110-1116, 2010. 
109. Del Rio D, Costa LG, Lean ME, and Crozier A. Polyphenols and health: what compounds are involved? Nutr Metab Cardiovasc Dis 20: 1-6, 2010.

110. Del Rio D, Stewart AJ, Mullen W, Burns J, Lean MEJ, Brighenti F, and Crozier A. HPLC-MS ${ }^{\mathrm{n}}$ analysis of phenolic compounds and purine alkaloids in green and black tea. J Agric Food Chem 52: 2807-2815, 2004.

111. Déprez S, Brezillon C, Rabot S, Philippe C, Mila I, Lapierre $\mathrm{C}$, and Scalbert A. Polymeric proanthocyanidins are catabolized by human colonic microflora into low-molecularweight phenolic acids. J Nutr 130: 2733-2738, 2000.

112. Desch S, Schmidt J, Kobler D, Sonnabend M, Eitel I, Sareban M, Rahimi K, Schuler G, and Thiele H. Effect of cocoa products on blood pressure: systematic review and meta-analysis. Am J Hypertens 23: 97-103, 2010.

113. Dinges DF. Cocoa flavanols, cerebral blood flow, cognition, and health: going forward. J Cardiovasc Pharmacol 47 Suppl 2: S221-S223, 2006.

114. Dohadwala MM, Hamburg NM, Holbrook M, Kim BH, Duess MA, Levit A, Titas M, Chung WB, Vincent FB, Caiano TL, Frame AA, Keaney JF, Jr., and Vita JA. Effects of Concord grape juice on ambulatory blood pressure in prehypertension and stage 1 hypertension. Am J Clin Nutr 92: 1052-1059, 2010.

115. Dohadwala MM, Holbrook M, Hamburg NM, Shenouda SM, Chung WB, Titas M, Kluge MA, Wang N, Palmisano J, Milbury PE, Blumberg JB, and Vita JA. Effects of cranberry juice consumption on vascular function in patients with coronary artery disease. Am J Clin Nutr 93: 934-940, 2011.

116. Dohadwala MM and Vita JA. Grapes and cardiovascular disease. J Nutr 139: 1788S-1793S, 2009.

117. Donnini S, Finetti F, Lusini L, Morbidelli L, Cheynier V, Barron D, Williamson G, Waltenberger J, and Ziche M. Divergent effects of quercetin conjugates on angiogenesis. Br J Nutr 95: 1016-1023, 2006.

118. Donovan JL, Manach C, Faulks RM, and Kroon PA. Absorption and metabolism of dietary secondary metabolites. In: Plant Secondary Metabolites. Occurrence, Structure and Role in the Human Diet, edited byCrozier A, Clifford MN, and Ashihara H. Oxford: Blackwell Publishing, 2006, pp. 303-351.

119. Donovan JL, Manach C, Rios L, Morand C, Scalbert A, and Rémésy C. Procyanidins are not bioavailable in rats fed a single meal containing a grape seed extract or the procyanidin dimer B3. Br J Nutr 87: 299-306, 2002.

120. Drapeau E, Mayo W, Aurousseau C, Le MM, Piazza PV, and Abrous DN. Spatial memory performances of aged rats in the water maze predict levels of hippocampal neurogenesis. Proc Natl Acad Sci U S A 100: 14385-14390, 2003.

121. Dueñas M, González-Manzano S, González-Paramás A, and Santos-Buelga C. Antioxidant evaluation of $\mathrm{O}$-methylated metabolites of catechin, epicatechin and quercetin. $J$ Pharm Biomed Anal 51: 443-449, 2010.

122. Duffy $\mathrm{R}$, Wiseman $\mathrm{H}$, and File SE. Improved cognitive function in postmenopausal women after 12 weeks of consumption of a soya extract containing isoflavones. Pharmacol Biochem Behav 75: 721-729, 2003.

123. Duffy SJ, Keaney JF, Jr., Holbrook M, Gokce N, Swerdloff PL, Frei B, and Vita JA. Short- and long-term black tea consumption reverses endothelial dysfunction in patients with coronary artery disease. Circulation 104: 151-156, 2001.

124. Duffy SJ, Vita JA, Holbrook M, Swerdloff PL, and Keaney JF, Jr. Effect of acute and chronic tea consumption on platelet aggregation in patients with coronary artery disease. Arterioscler Thromb Vasc 21: 1084-1089, 2001.

125. DuPont MS, Day AJ, Bennet RN, Mellon FA, and Kroon PA. Absorption of kaempferol from endive, source of kaempferol-3-glucuronide, in humans. Eur J Clin Nutr 58: 947-954, 2004.

126. Duthie SJ and Dobson VL. Dietary flavonoids protect human colonocyte DNA from oxidative attack in vitro. Eur J Nutr 38: 28-34, 1999.

127. Duthie SJ, Jenkinson AM, Crozier A, Mullen W, Pirie L, Kyle J, Yap LS, Christen P, and Duthie GG. The effects of cranberry juice consumption on antioxidant status and biomarkers relating to heart disease and cancer in healthy human volunteers. Eur J Nutr 45: 113-122, 2006.

128. Eeckhaut E, Struijs K, Possemiers, S, Vincken JP, Keukeleire $\mathrm{DD}$, and Verstraete W. Metabolism of lignan macromolecule into eneterolignans in the gastrointestinal lumen as determined in the simulator of the human intestinal microbial ecosystem. J Agric Food Chem 56: 4806-4812, 2008.

129. El Mohsen MA, Marks J, Kuhnle G, Moore K, Debnam E, Kaila Srai S, Rice-Evans C, and Spencer JP. Absorption, tissue distribution and excretion of pelargonidin and its metabolites following oral administration to rats. $\mathrm{Br} J \mathrm{Nutr}$ 95: 51-58, 2006.

130. Elks CM, Reed SD, Mariappan N, Shukitt-Hale B, Joseph JA, Ingram DK, and Francis J. A blueberry-enriched diet attenuates nephropathy in a rat model of hypertension via reduction in oxidative stress. PLoS One 6: e24028, 2011.

131. Engler MB, Engler MM, Chen CY, Malloy MJ, Browne A, Chiu EY, Kwak HK, Milbury P, Paul SM, Blumberg J, and Mietus-Snyder ML. Flavonoid-rich dark chocolate improves endothelial function and increases plasma epicatechin concentrations in healthy adults. J Am Coll Nutr 23: 197-204, 2004.

132. Erlund I, Koli R, Alfthan G, Marniemi J, Puukka P, Mustonen P, Mattila P, and Jula A. Favorable effects of berry consumption on platelet function, blood pressure, and HDL cholesterol. Am J Clin Nutr 87: 323-331, 2008.

133. Espín JC, García-Conesa MT, and Tomás-Barberán FA. Nutraceuticals: facts and fiction. Phytochemistry 68: 29863008, 2007.

134. Espín JC, González-Barrio R, Cerdá B, López-Bote C, Rey AI, and Tomás-Barberán FA. Iberian pig as a model to clarify obscure points in the bioavailability and metabolism of ellagitannins in humans. J Agric Food Chem 55: 1047610485, 2007.

135. Estruch R, Sacanella E, Mota F, Chiva-Blanch G, Antunez E, Casals E, Deulofeu R, Rotilio D, Andres-Lacueva C, Lamuela-Raventos RM, de Gaetano G, and Urbano-Marquez A. Moderate consumption of red wine, but not gin, decreases erythrocyte superoxide dismutase activity: a randomised cross-over trial. Nutr Metab Cardiovasc Dis 21: 4653, 2011.

136. Faridi Z, Njike VY, Dutta S, Ali A, and Katz DL. Acute dark chocolate and cocoa ingestion and endothelial function: a randomized controlled crossover trial. Am J Clin Nutr 88: 58-63, 2008.

137. Favero A, Parpinel M, and Franceschi S. Diet and risk of breast cancer: major findings from an Italian case-control study. Biomed Pharmacother 52: 109-115, 1998.

138. Fehr M, Galliard-Grigioni KS, and Reinhart WH. Influence of acute alcohol exposure on hemorheological parameters and platelet function in vivo and in vitro. Clin Hemorheol Microcirc 39: 351-358, 2008. 
139. Felgines C, Talavéra S, Gonthier M-P, Texier O, Scalbert A, Lamaison JL, and Rémésy C. Strawberry anthocyanins are recovered in urine as glucuro- and sulfoconjugates in humans. J Nutr 133: 1269-1301, 2003.

140. Feskanich D, Ziegler RG, Michaud DS, Giovannucci EL, Speizer FE, Willett WC, and Colditz GA. Prospective study of fruit and vegetable consumption and risk of lung cancer among men and women. J Natl Cancer Inst 92: 1812-1823, 2000.

141. File SE, Jarrett N, Fluck E, Duffy R, Casey K, and Wiseman H. Eating soya improves human memory. Psychopharmacology 157: 430-436, 2001.

142. Fisher ND, Sorond FA, and Hollenberg NK. Cocoa flavanols and brain perfusion. J Cardiovasc Pharmacol 47 Suppl 2: S210-S214, 2006.

143. Flammer AJ, Hermann F, Sudano I, Spieker L, Hermann M, Cooper KA, Serafini M, Luscher TF, Ruschitzka F, Noll G, and Corti R. Dark chocolate improves coronary vasomotion and reduces platelet reactivity. Circulation 116: 2376-2382, 2007.

144. Forester SC and Waterhouse AL. Gut metabolites of anthocyanins, gallic acid, 3-O-methylgallic acid, and 2,4,6trihydroxybenzaldehyde, inhibit cell proliferation of Caco-2 cells. J Agric Food Chem 58: 5320-5327, 2010.

145. Fournier LR, Ryan Borchers TA, Robison LM, Wiediger M, Park JS, Chew BP, McGuire MK, Sclar DA, Skaer TL, and Beerman KA. The effects of soy milk and isoflavone supplements on cognitive performance in healthy, postmenopausal women. J Nutr Health Aging 11: 155-164, 2007.

146. Fraga CG, Actis-Goretta L, Ottaviani JI, Carrasquedo F, Lotito SB, Lazarus S, Schmitz HH, and Keen CL. Regular consumption of a flavanol-rich chocolate can improve oxidant stress in young soccer players. Clin Dev Immunol 12: 11-17, 2005.

147. Fraga CG, Litterio MC, Prince PD, Calabro V, Piotrkowski B, and Galleano M. Cocoa flavanols: effects on vascular nitric oxide and blood pressure. J Clin Biochem Nutr 48: 6367, 2011.

148. Fraga CG and Oteiza PI. Dietary flavonoids: role of (-)epicatechin and related procyanidins in cell signaling. Free Radic Biol Med 51: 813-823, 2011.

149. Francis ST, Head K, Morris PG, and Macdonald IA. The effect of flavanol-rich cocoa on the fMRI response to a cognitive task in healthy young people. J Cardiovasc Pharmacol 47 Suppl 2: S215-S220, 2006.

150. Gao HM and Hong JS. Why neurodegenerative diseases are progressive: uncontrolled inflammation drives disease progression. Trends Immunol 29: 357-365, 2008.

151. Gee JM, DuPont SM, Day AJ, Plumb GW, Williamson G, and Johnson IT. Intestinal transport of quercetin glycosides in rats involves both deglycosylation and interaction with the hexose transport pathway. J Nutr 130: 2765-2771, 2000.

152. Giovannelli L, Pitozzi V, Luceri C, Giannini L, Toti S, Salvini S, Sera F, Souquet JM, Cheynier V, Sofi F, Mannini L, Gori AM, Abbate R, Palli D, and Dolara P. Effects of dealcoholised wines with different polyphenol content on DNA oxidative damage, gene expression of peripheral lymphocytes, and haemorheology: an intervention study in post-menopausal women. Eur J Nutr 50: 19-29, 2011.

153. Glässer G, Graefe EU, Struck F, Veit M, and Gebhardt R. Comparison of antioxidative capacities and inhibitory effects on cholesterol biosynthesis of quercetin and potential metabolites. Phytomedicine 9: 33-40, 2002.
154. Goldberg DM, Yan J, and Soleas GJ. Absorption of three wine-related polyphenols in three different matrices by healthy subjects. Clin Biochem 36: 79-87, 2003.

155. Gómez-Ruiz JA, Leake DS, and Ames JM. In vitro antioxidant activity of coffee compounds and their metabolites. $J$ Agric Food Chem 55: 6962-6969, 2007.

156. Gonthier MP, Donovan JL, Texier O, Felgines C, Remesy C, and Scalbert A. Metabolism of dietary procyanidins in rats. Free Radic Biol Med 35: 837-844, 2003.

157. González CA, Pera G, Agudo A, Bueno-de-Mesquita HB, Ceroti M, Boeing H, Schulz M, Del Giudice G, Plebani M, Carneiro F, Berrino F, Sacerdote C, Tumino R, Panico S, Berglund G, Siman H, Hallmans G, Stenling R, Martinez C, Dorronsoro M, Barricarte A, Navarro C, Quiros JR, Allen N, Key TJ, Bingham S, Day NE, Linseisen J, Nagel G, Overvad K, Jensen MK, Olsen A, Tjonneland A, Buchner FL, Peeters PH, Numans ME, Clavel-Chapelon F, BoutronRuault MC, Roukos D, Trichopoulou A, Psaltopoulou T, Lund E, Casagrande C, Slimani N, Jenab M, and Riboli E. Fruit and vegetable intake and the risk of stomach and oesophagus adenocarcinoma in the European Prospective Investigation into Cancer and Nutrition (EPIC-EURGAST). Int J Cancer 118: 2559-2566, 2006.

158. González-Barrio R, Borges G, Mullen W, and Crozier A. Bioavailability of raspberry anthocyanins and ellagitannins following consumption of raspberries by healthy humans and subjects with an ileostomy. J Agric Food Chem 58: 3933$3939,2010$.

159. González-Barrio R, Edwards CA, and Crozier A. Colonic catabolism of ellagitannins, ellagic acid and raspberry anthocyanins: in vivo and in vitro studies. Drug Met Dispos 39: 1680-1688, 2011.

160. González-Sarrías A, Azorín-Ortuño M, Yáñez-Gascón MJ, Tomás-Barberán FA, García-Conesa MT, and Espín JC. Dissimilar in vitro and in vivo effects of ellagic acid and its microbiota-derived metabolites, urolithins, on the cytochrome P450 1A1. J Agric Food Chem 57: 5623-5632, 2009.

161. González-Sarrías A, Espín JC, Tomás-Barberán FA, and García-Conesa MT. Gene expression, cell cycle arrest and MAPK signaling regulation in Caco-2 cells exposed to ellagic acid and its metabolites, urolithins. Mol Nutr Food Res 53: 686-698, 2009.

162. González-Sarrías A, Giménez-Bastida JA, García-Conesa MT, Gómez-Sánchez MB, García-Talavera NV, Gil-Izquierdo A, Sánchez-Alvarez C, Fontana-Compiano LO, Morga-Egea JP, Pastor-Quirante FA, Martínez-Díaz F, Tomás-Barberán FA, and Espín JC. Occurrence of urothins, gut microbiota ellagic acid metabolites and proliferation markers expression response in the human prostate gland after consumption of walnuts and pomegranate juice. Mol Nutr Food Res 54: 311-322, 2010.

163. González-Sarrías A, Larrosa M, Tomás-Barberán FA, Dolara P, and Espín JC. NF- $\kappa$ B-dependent anti-inflammatory activity of urolithins, gut microbiota ellagic acid-derived metabolites, in human colonic fibroblasts. Br J Nutr 104: 503-512, 2010.

164. Gosnay SL, Bishop JA, New SA, Catterick J, and Clifford MN. Estimation of the mean intakes of fourteen classes of dietary phenolics in a population of young British women aged 20-30 years. Proc Nutr Soc 61: 125A, 2002.

165. Grassi D, Desideri G, Necozione S, Lippi C, Casale R, Properzi G, Blumberg JB, and Ferri C. Blood pressure is reduced and insulin sensitivity increased in glucoseintolerant, hypertensive subjects after 15 days of 
consuming high-polyphenol dark chocolate. J Nutr 138: 1671-1676, 2008.

166. Grassi D, Lippi C, Necozione S, Desideri G, and Ferri C. Short-term administration of dark chocolate is followed by a significant increase in insulin sensitivity and a decrease in blood pressure in healthy persons. Am J Clin Nutr 81: 611614, 2005.

167. Grassi D, Mulder TP, Draijer R, Desideri G, Molhuizen HO, and Ferri $\mathrm{C}$. Black tea consumption dose-dependently improves flow-mediated dilation in healthy males. J Hypertens 27: 774-781, 2009.

168. Grassi D, Necozione S, Lippi C, Croce G, Valeri L, Pasqualetti P, Desideri G, Blumberg JB, and Ferri C. Cocoa reduces blood pressure and insulin resistance and improves endothelium-dependent vasodilation in hypertensives. Hypertension 46: 398-405, 2005.

169. Gredel S, Grad C, Rechkemmer G, and Watzl B. Phytoestrogens and phytoestrogen metabolites differentially modulate immune parameters in human leukocytes. Food Chem Toxicol 46: 3691-3696, 2008.

170. Grimm T, Schäfer A, and Högger P. Antioxidant activity and inhibition of matrix metalloproteinases by metabolites of maritime pine bark extract (pycnogenol). Free Radic Biol Med 36: 811-822, 2004.

171. Grumer HD. Formation of hippuric acid from phenylalanine labelled with carbon-14 in phenylketonuric subjects. Nature 189: 63-64, 1961.

172. Guan S, Ge D, Liu TQ, Ma XH, and Cui ZF. Protocatechuic acid promotes cell proliferation and reduces basal apoptosis in cultured neural stem cells. Toxicol In Vitro 23: 201-208, 2009.

173. Guan S, Jiang B, Bao YM, and An LJ. Protocatechuic acid suppresses $\mathrm{MPP}^{+}$-induced mitochondrial dysfunction and apoptotic cell death in PC12 cells. Food Chem Toxicol 44: 1659-1666, 2006

174. Guarda E, Godoy I, Foncea R, Perez DD, Romero C, Venegas $R$, and Leighton $F$. Red wine reduces oxidative stress in patients with acute coronary syndrome. Int J Cardiol 104: 35-38, 2005.

175. Hagl S, Deusser H, Soyalan B, Janzowski C, Will F, Dietrich $\mathrm{H}$, Albert FW, Rohner S, and Richling E. Colonic availability of polyphenols and D-(-)-quinic acid after apple smoothie consumption. Mol Nutr Food Res 55: 368-377, 2011.

176. Hakim IA, Harris RB, Brown S, Chow HH, Wiseman S, Agarwal S, and Talbot W. Effect of increased tea consumption on oxidative DNA damage among smokers: a randomized controlled study. J Nutr 133: 3303S-3309S, 2003.

177. Hamajima N, Tajima K, Tominaga S, Matsuura A, Kuwabara $\mathrm{M}$, and Okuma $\mathrm{K}$. Tea polyphenol intake and changes in serum pepsinogen levels. Jpn J Cancer Res 90: 136-143, 1999.

178. Hansen AS, Marckmann P, Dragsted LO, Finne Nielsen IL, Nielsen SE, and Gronbaek M. Effect of red wine and red grape extract on blood lipids, haemostatic factors, and other risk factors for cardiovascular disease. Eur J Clin Nutr 59: 449-455, 2005.

179. Haque AM, Hashimoto M, Katakura M, Tanabe Y, Hara Y, and Shido O. Long-term administration of green tea catechins improves spatial cognition learning ability in rats. $J$ Nutr 136: 1043-1047, 2006.

180. Hartman RE, Shah A, Fagan AM, Schwetye KE, Parsadanian $\mathrm{M}$, Schulman RN, Finn MB, and Holtzman DM. Pomegranate juice decreases amyloid load and improves behavior in a mouse model of Alzheimer's disease. Neurobiol Dis 24: 506-515, 2006.

181. Hashimoto M, Kim S, Eto M, Iijima K, Ako J, Yoshizumi M, Akishita M, Kondo K, Itakura $\mathrm{H}$, Hosoda $\mathrm{K}$, Toba $\mathrm{K}$, and Ouchi Y. Effect of acute intake of red wine on flow-mediated vasodilatation of the brachial artery. Am J Cardiol 88: 1457-1560, A9, 2001.

182. Hassellund SS, Flaa A, Sandvik L, Kjeldsen SE, and Rostrup M. Effects of anthocyanins on blood pressure and stress reactivity: a double-blind randomized placebo-controlled crossover study. J Hum Hypertens 26: 396-404, 2012.

183. He FJ, Nowson CA, Lucas M, and MacGregor GA. Increased consumption of fruit and vegetables is related to a reduced risk of coronary heart disease: meta-analysis of cohort studies. J Hum Hypertens 21: 717-728, 2007.

184. Heinonen S-M, Nurmi T, Liukkonen K, Poutanen K, Wähälä K, Deyama T, Nishibe S, and Adlercreutz H. In vitro metabolism of plant lignans: new precursors of mammalian lignans enterolactone and enterodiol. J Agric Food Chem 49: 3178-3186, 2001.

185. Heinonen S-M, Wähälä K, and Adlercreutz H. Identification of urinary metabolites of the red clover isoflavones formononetin and biochanin A in human subjects. J Agric Food Chem 52: 6802-6809, 2004.

186. Heinonen S-M, Wähälä K, Liukkonen K-H, Aura AM, Poutanen $\mathrm{K}$, and Adlercreutz $\mathrm{H}$. Studies of the in vitro intestinal metabolism of isoflavones aid in the identification of their urinary metabolites. J Agric Food Chem 52: 26402646, 2004.

187. Heiss C, Dejam A, Kleinbongard P, Schewe T, Sies H, and Kelm M. Vascular effects of cocoa rich in flavan-3-ols. JAMA 290: 1030-1031, 2003.

188. Heiss C, Finis D, Kleinbongard P, Hoffmann A, Rassaf T, Kelm M, and Sies H. Sustained increase in flow-mediated dilation after daily intake of high-flavanol cocoa drink over 1 week. J Cardiovasc Pharmacol 49: 74-80, 2007.

189. Heiss C, Jahn S, Taylor M, Real WM, Angeli FS, Wong ML, Amabile N, Prasad M, Rassaf T, Ottaviani JI, Mihardja S, Keen CL, Springer ML, Boyle A, Grossman W, Glantz SA, Schroeter $\mathrm{H}$, and Yeghiazarians $\mathrm{Y}$. Improvement of endothelial function with dietary flavanols is associated with mobilization of circulating angiogenic cells in patients with coronary artery disease. J Am Coll Cardiol 56: 218-224, 2010.

190. Heiss C, Kleinbongard P, Dejam A, Perre S, Schroeter H, Sies $\mathrm{H}$, and Kelm M. Acute consumption of flavanol-rich cocoa and the reversal of endothelial dysfunction in smokers. J Am Coll Cardiol 46: 1276-1283, 2005.

191. Henderson VW. Estrogen-containing hormone therapy and Alzheimer's disease risk: understanding discrepant inferences from observational and experimental research. Neuroscience 138: 1031-1039, 2006.

192. Henning SM, Niu Y, Lee NH, hames GD, Minutti RR, Wang $\mathrm{H}$, Go VL, and Heber D. Bioavailability and antioxidant activity of tea flavanols after consumption of green tea, black tea, or a green tea extract supplement. Am J Clin Nutr 80: 1558-1564, 2004.

193. Hertog MG, Sweetnam PM, Fehily AM, Elwood PC, and Kromhout D. Antioxidant flavonols and ischemic heart disease in a Welsh population of men: the Caerphilly Study. Am J Clin Nutr 65: 1489-1494, 1997.

194. Hertog MGL, Hollman PCH, and Katan MB. Content of potentially anticarcinogenic flavonoids of 28 vegetables and 9 fruits commonly consumed in The Netherlands. $J$ Agric Food Chem 41: 1242-1246, 1993. 
195. Hertog MGL, Hollman PCH, and van de Putte B. Content of potentially anticarcinogenic flavonoids of tea infusions, wines and fruit juices in The Netherlands. J Agric Food Chem 40: 2379-2383, 1992.

196. Hijmering ML, de Lange DW, Lorsheyd A, Kraaijenhagen RJ, and van de Wiel A. Binge drinking causes endothelial dysfunction, which is not prevented by wine polyphenols: a small trial in healthy volunteers. Neth J Med 65: 29-35, 2007.

197. Hirsh J. Hyperactive platelets and complications of coronary artery disease. $N$ Engl J Med 316: 1543-1544, 1987.

198. Ho SC, Chan AS, Ho YP, So EK, Sham A, Zee B, and Woo JL. Effects of soy isoflavone supplementation on cognitive function in Chinese postmenopausal women: a double-blind, randomized, controlled trial. Menopause 14: 489-499, 2007.

199. Ho Y, Lee Y-L, and Hsu K-Y. Determination of (+)-catechin in plasma by high-performance liquid chromatography using fluorescence detection. J Chromatogr B 665: 383-389, 1995.

200. Hodgson JM, Burke V, and Puddey IB. Acute effects of tea on fasting and postprandial vascular function and blood pressure in humans. J Hypertens 23: 47-54, 2005.

201. Hodgson JM, Chan SY, Puddey IB, Devine A, Wattanapenpaiboon N, Wahlqvist ML, Lukito W, Burke V, Ward NC, Prince RL, and Croft KD. Phenolic acid metabolites as biomarkers for tea- and coffee-derived polyphenol exposure in human subjects. Br J Nutr 91: 301-306, 2004.

202. Hodgson JM, Morton LW, Puddey IB, Beilin LJ, and Croft KD. Gallic acid metabolites are markers of black tea intake in humans. J Agric Food Chem 48: 2276-2280, 2000.

203. Hodgson JM, Puddey IB, Burke V, Beilin LJ, and Jordan N. Effects on blood pressure of drinking green and black tea. $J$ Hypertens 17: 457-463, 1999.

204. Hodgson JM, Puddey IB, Burke V, Beilin LJ, Mori TA, and Chan SY. Acute effects of ingestion of black tea on postprandial platelet aggregation in human subjects. Br J Nutr 87: 141-145, 2002.

205. Hodgson JM, Puddey IB, Burke V, Watts GF, and Beilin LJ. Regular ingestion of black tea improves brachial artery vasodilator function. Clin Sci 102: 195-201, 2002.

206. Hodgson JM, Puddey IB, Mori TA, Burke V, Baker RI, and Beilin LJ. Effects of regular ingestion of black tea on haemostasis and cell adhesion molecules in humans. Eur J Clin Nutr 55: 881-886, 2001.

207. Hoffman JR, Donato A, and Robbins SJ. Ginkgo biloba promotes short-term retention of spatial memory in rats. Pharmacol Biochem Behav 77: 533-539, 2004.

208. Hoikkala A, Mustonen E, Saastamoinen I. Jokela T, Taponen J, Saloniemi H, and Wähälä K. High levels of equol in organic skimmed Finnish cow milk. Mol Nutr Food Res 51: 782-786, 2007.

209. Holt RR, Actis-Goretta L, Momma TY, and Keen CL. Dietary flavanols and platelet reactivity. J Cardiovasc Pharmacol 47 Suppl 2: S187-S196; discussion S206-S209, 2006.

210. Holt RR, Lazarus SA, Sullards, MC, Zhu QY, Schramm DD, Hammerstone JF, Fraga CG, Schmitz HH, and Keen CL. Procyanidin dimer B2 [epicatechin-(4 $\beta$-8)-epicatechin] in human plasma after the consumption of a flavanol-rich cocoa. Am J Clin Nutr 76: 798-804, 2002.

211. Hooper L, Kroon PA, Rimm EB, Cohn JS, Harvey I, Le Cornu KA, Ryder JJ, Hall WL, and Cassidy A. Flavonoids, flavonoid-rich foods, and cardiovascular risk: a metaanalysis of randomized controlled trials. Am J Clin Nutr 88: 38-50, 2008.
212. Hooper L, Madhavan G, Tice JA, Leinster SJ, and Cassidy A. Effects of isoflavones on breast density in pre- and postmenopausal women: a systematic review and meta-analysis of randomized controlled trials. Hum Reprod Update 16: 745-760, 2010.

213. Hosoda K, Furuta T, and Ishii K. Metabolism and disposition of iosflavone conjugated metabolites in humans after ingestion of Kinako. Drug Metab Disp 39: 1762-1767, 2011.

214. Howes JB, Bray K, Lorenz L, Smerdely P, and Howes LG. The effects of dietary supplementation with isoflavones from red clover on cognitive function in postmenopausal women. Climacteric 7: 70-77, 2004.

215. Huang J, de Paulis T, and May JM. Antioxidant effects of dihydrocaffeic acid in human EA.hy926 endothelial cells. J Nutr Biochem 15: 722-729, 2004.

216. Ishisaka A, Ichikawa S, Sakakibara H, Piskula MK, Nakamura T, Kato Y, Ito M, Miyamoto K, Tsuji A, Kawai Y, and Terao J. Accumulation of orally administered quercetin in brain tissue and its antioxidative effects on rats. Free Radic Biol Med 51: 1329-1336, 2011.

217. Ito $H$. Metabolites of the ellagitannin geraniin and their antioxidant activities. Planta Med 77: 1110-1115, 2011.

218. Jaganath IB and Crozier A. Overview of health promoting compounds in fruits and vegetables. In: Phenolic Compounds of Plant Origin and Health: The Biochemistry Behind Their Nutritional and Pharmacological Value, edited by Fraga C. Chichester, United Kingdom: Wiley, 2009, pp. 1-48.

219. Jaganath IB and Crozier A. Flavonoid metabolism. In: Plant Metabolism and Biotechnology, edited by Ashihara H, Crozier A, and Komamine A. Chichester, United Kingdom: Wiley, 2011, pp. 285-312.

220. Jaganath IB, Mullen W, Edwards CA, and Crozier A. The relative contribution of the small and large intestine to the absorption and metabolism of rutin in man. Free Radic Res 40: 1035-1046, 2006.

221. Jaganath IB, Mullen W, Lean ME, Edwards CA, and Crozier A. In vitro catabolism of rutin by human fecal bacteria and the antioxidant capacity of its catabolites. Free Radic Biol Med 47: 1180-1189, 2009.

222. Janle EM, Lila MA, Grannan M, Wood W, Higgins A, Yousef GG, Rogers R, Kim H, Jackson GS, Ho L, and Weaver CM. Pharmacokinetics and tissue distribution of ${ }^{14} \mathrm{C}$-labeled grape polyphenols in the periphery and central nervous system following oral administration. J Med Food 13: 926-933, 2010.

223. Jee SH, He J, Appel LJ, Whelton PK, Suh I, and Klag MJ. Coffee consumption and serum lipids: a meta-analysis of randomized controlled clinical trials. Am J Epidemiol 153: 353-362, 2001.

224. Jee SH, He J, Whelton PK, Suh I, and Klag MJ. The effect of chronic coffee drinking on blood pressure: a metaanalysis of controlled clinical trials. Hypertension 33: 647652, 1999.

225. Joannou GE, Kelly G, Reeder AY, Waring M, and Nelson C. A urinary profile study of dietary phytoestrogens. The identification and mode of metabolism of new isoflavonoids. J Steroid Biochem Mol Biol 54: 167-184, 1995.

226. Jochmann N, Lorenz M, Krosigk A, Martus P, Bohm V, Baumann G, Stangl K, and Stangl V. The efficacy of black tea in ameliorating endothelial function is equivalent to that of green tea. Br J Nutr 99: 863-868, 2008.

227. Johnsen NF, Hausner H, Olsen A, Tetens I, Christensen J, Knudsen KE, Overvad $\mathrm{K}$, and Tjønneland A. Intake of whole grains and vegetables determines the plasma en- 
terolactone concentration of Danish women. J Nutr 134: 2691-2697, 2004.

228. Johnson K, Sharp P, Clifford MN, and Morgan L. Dietary polyphenols decrease glucose uptake by human intestinal Caco-2 cells. FEBS Lett 579: 1653-1657, 2005.

229. Joseph JA, Shukitt-Hale B, Denisova NA, Bielinski D, Martin A, McEwen JJ, and Bickford PC. Reversals of agerelated declines in neuronal signal transduction, cognitive, and motor behavioral deficits with blueberry, spinach, or strawberry dietary supplementation. J Neurosci 19: 81148121, 1999.

230. Joseph JA, Shukitt-Hale B, Denisova NA, Prior RL, Cao G, Martin A, Taglialatela G, and Bickford PC. Long-term dietary strawberry, spinach, or vitamin $\mathrm{E}$ supplementation retards the onset of age-related neuronal signal-transduction and cognitive behavioral deficits. J Neurosci 18: 80478055, 1998.

231. Joshipura KJ, Hu FB, Manson JE, Stampfer MJ, Rimm EB, Speizer FE, Colditz G, Ascherio A, Rosner B, Spiegelman D, and Willett WC. The effect of fruit and vegetable intake on risk for coronary heart disease. Ann Intern Med 134: 11061114, 2001.

232. Joy S, Siow RC, Rowlands DJ, Becker M, Wyatt AW, Aaronson PI, Coen CW, Kallo I, Jacob R, and Mann GE. The isoflavone equol mediates rapid vascular relaxation: $\mathrm{Ca}^{2+}$ independent activation of endothelial nitric-oxide synthase/ Hsp90 involving ERK1/2 and Akt phosphorylation in human endothelial cells. J Biol Chem 281: 27335-27345, 2006.

233. Kahle K, Huemmer W, Kempf M, Scheppach W, Erk T, and Richling E. Polyphenols are extensively metabolized in the human gastrointestinal tract after apple juice consumption. J Agric Food Chem 55: 10695-10614, 2007.

234. Kahle K, Kempf M, Schreier P, Scheppach W, Schrenk D, Kautenburger T, Hecker D, Huemmer W, Ackermann M, and Richling E. Intestinal transit and systemic metabolism of apple polyphenols. Eur J Nutr 50: 507-522, 2011.

235. Kahle K, Kraus M, Scheppach W, Ackermann M, Ridder F, and Richling, E. Studies on apple and blueberry fruit constituents: do the polyphenols reach the colon after ingestion? Mol Nutr Food Res 50: 418-423, 2006.

236. Kahle K, Kraus M, Scheppach W, and Richling E. Colonic availability of apple polyphenols - a study in ileostomy subjects. Mol Nutr Food Res 49: 1143-1150, 2005.

237. Kalt W, Blumberg JB, McDonald JE, Vinqvist-Tymchuk MR, Fillmore SA, Graf BA, O'Leary JM, and Milbury PE. Identification of anthocyanins in the liver, eye, and brain of blueberry-fed pigs. J Agric Food Chem 56: 705-712, 2008.

238. Kamiyama M, Kishimoto $Y$, Tani M, Utsunomiya $K$, and Kondo K. Effects of equol on oxidized low-density lipoprotein-induced apoptosis in endothelial cells. J Atheroscler Thromb 16: 239-249, 2009.

239. Kaplan M, Hayek T, Raz A, Coleman R, Dornfeld L, Vaya J, and Aviram M. Pomegranate juice supplementation to atherosclerotic mice reduces macrophage lipid peroxidation, cellular cholesterol accumulation and development of atherosclerosis. J Nutr 131: 2082-2089, 2001.

240. Karatzi K, Papamichael C, Aznaouridis K, Karatzis E, Lekakis J, Matsouka C, Boskou G, Chiou A, Sitara M, Feliou G, Kontoyiannis D, Zampelas A, and Mavrikakis M. Constituents of red wine other than alcohol improve endothelial function in patients with coronary artery disease. Coron Artery Dis 15: 485-490, 2004.

241. Kasimsetty SG, Bialonska D, Reddy MK, Thornton C, Willett KL, and Ferreira D. Effects of pomegranate chemical constituents/intestinal microbial metabolites on CYP1B1 in 22Rv1 prostate cancer cells. J Agric Food Chem 57: 1063610644, 2009.

242. Kaspar KL, Park JS, Brown CR, Mathison BD, Navarre DA, and Chew BP. Pigmented potato consumption alters oxidative stress and inflammatory damage in men. J Nutr 141: 108-111, 2011.

243. Kawai Y, Nishikawa, T, Shiba Y, Saito S, Murota K, Shibata N, Kobayashi M, Kanayama M, Uchida K, and Terao J. Macrophage as a target of quercetin glucuronides in human atherosclerotic arteries. Implication in the anti-atherosclerotic mechanism of dietary flavonoids. J Biol Chem 283: 9424-9434, 2008.

244. Kawai Y, Tanaka H, Murota K, Naito M, and Terao J. (- )Epicatechin gallate accumulates in foamy macrophages in human atherosclertic aorta: implication in the anti-atherosclerotic actions of tea catechins. Biochem Biophys Res Comm 374: 527-532, 2008.

245. Kechagias S, Zanjani S, Gjellan S, Leinhard OD, Kihlberg J, Smedby O, Johansson L, Kullberg J, Ahlstrom H, Lindstrom T, and Nystrom FH. Effects of moderate red wine consumption on liver fat and blood lipids: a prospective randomized study. Ann Med 43: 545-554, 2011.

246. Kempermann G, Kuhn HG, and Gage FH. Experience-induced neurogenesis in the senescent dentate gyrus. J Neurosci 18: 3206-3212, 1998.

247. Kida T, Suzuki M, Matsumoto N, Nanjo F, and Hara Y. Identification of biliary metabolites of (-)-epigallocatechin gallate in rats. J Agric Food Chem 48: 4151-4155, 2000.

248. Kikura M, Levy JH, Safon RA, Lee MK, and Szlam F. The influence of red wine or white wine intake on platelet function and viscoelastic property of blood in volunteers. Platelets 15: 37-41, 2004.

249. Kilkkinen A, Stumpf K, Pietinen P, Valsta LM, Tapanainen $\mathrm{H}$, and Adlercreutz $\mathrm{H}$. Determinants of serum enterolactone concentration. Am J Clin Nutr 73: 1094-1100, 2001.

250. Klaus A and Birchmeier W. Wnt signalling and its impact on development and cancer. Nat Rev Cancer 8: 387-398, 2008.

251. Koga $\mathrm{T}$ and Meydani M. Effect of plasma metabolites of (+)-catechin and quercetin on monocyte adhesion to human aortic endothelial cells. Am J Clin Nutr 73: 941-948, 2001.

252. Kohri T, Nanjo F, Suziki M, Seto R, Matsumoto N, Yamakawa M, Hojo H, Hara Y, Desai D, Amin S, Conaway CC, and Chung FL. Synthesis of $(-)-\left[4-^{3} \mathrm{H}\right]$ epigallocatechin gallate and its metabolic fate in rats after intravenous administration. J Agric Food Chem 49: 1042-1048, 2001.

253. Kottra G and Daniel H. Flavonoid glycosides are not transported by the human $\mathrm{Na}^{+}$/glucose transporter when expressed in Xenopus laevis oocytes, but effectively inhibit electrogenic glucose uptake. J Pharmacol Exp Ther 322: 829835, 2007.

254. Krafczyk N and Glomb MA. Characterization of phenolic compounds in rooibos tea. J Agric Food Chem 56: 3368-3376, 2008.

255. Kreijkamp-Kaspers S, Kok L, Grobbee DE, de Haan EH, Aleman A, Lampe JW, and van der Schouw YT. Effect of soy protein containing isoflavones on cognitive function, bone mineral density, and plasma lipids in postmenopausal women: a randomized controlled trial. JAMA 292: 65-74, 2004.

256. Kreuz A, Joubert E, Waldmann $\mathrm{KH}$, and Ternes W. Aspalathin, a flavonoid in Aspalathus linearis (rooibos), is 
absorbed by pig intestine as a C-glycoside. Nutr Res 28: 690-701, 2008.

257. Krikorian R, Nash TA, Shidler MD, Shukitt-Hale B, and Joseph JA. Concord grape juice supplementation improves memory function in older adults with mild cognitive impairment. Br J Nutr 103: 730-734, 2010.

258. Krikorian R, Shidler MD, Nash TA, Kalt W, VinqvistTymchuk MR, Shukitt-Hale B, and Joseph JA. Blueberry supplementation improves memory in older adults. J Agric Food Chem 58: 3996-4000, 2010.

259. Kritz-Silverstein D, Von MD, Barrett-Connor E, and Bressel MA. Isoflavones and cognitive function in older women: the Soy and Postmenopausal Health In Aging (SOPHIA) Study. Menopause 10: 196-202, 2003.

260. Kuhn HG, Dickinson-Anson H, and Gage FH. Neurogenesis in the dentate gyrus of the adult rat: age-related decrease of neuronal progenitor proliferation. J Neurosci 16: 2027-2033, 1996.

261. Kuhnert N, Clifford MN, and Muller A. Oxidative cascade reactions yielding polyhydroxy-theaflavins and theacitrins in the formation of black tea thearubigins: evidence by tandem LC-MS. Food Funct 1: 180-199, 2010.

262. Kuhnert N, Dryman JW, Obuchowicz J, Clifford MN, and Witt M. Mass spectrometric characterization of black tea thearubigins leading to an oxidative cascade hypothesis for thearubigin formation. Rapid Comm Mass Spectrom 24: 3387-3404, 2010.

263. Kurowska EM, Spence JD, Jordan J, Wetmore S, Freeman DJ, Piche LA, and Serratore P. HDL-cholesterol-raising effect of orange juice in subjects with hypercholesterolemia. Am J Clin Nutr 72: 1095-1100, 2000.

264. La Vecchia C, Chatenoud L, Franceschi S, Soler M, Parazzini F, and Negri E. Vegetables and fruit and human cancer: update of an Italian study. Int J Cancer 82: 151-152, 1999.

265. Lai M-Y, Hou Y-C, Hsiu S-L, Chen C-C, and Chao P-D-L. Relative flavone bioavailability of Scutellariae Radix between traditional decoction and commercial power preparation in humans. J Food Drug Anal 10: 75-80, 2002.

266. Lambert JD, Rice JE, Hong J, Hou Z, and Yang CS. Synthesis and biological activity of the tea catechin metabolites, M4 and M6 and their methoxy-derivatives. Bioorg Med Chem Lett 15: 873-876, 2005.

267. Landete JM. Ellagitannins, ellagic acid and their derived metabolites: a review about source, metabolism, functions and health. Food Res Int 44: 1150-1160, 2011.

268. Langcake P and Pryce RJ. A new class of phytoalexins from grapevines Experientia 33: 151-152, 1977.

269. Larrosa M, García-Conesa MT, Espin JC, and Tomás-Barberán FA. Bioavailability and metabolism of ellagic acid and ellagitannins. In: Flavonoids and Related Compounds: Bioavailability and Function, edited by Spencer JPE and Crozier A. Oxidative Stress and Disease, Vol. 30, edited by Packer L and Cadenas H. Boca Raton, FL: CRC Press, 2012, pp. 183-199.

270. Larrosa M, González-Sarrías A, García-Conesa MT, TomásBarberán FA, and Espín JC. Urolithins, ellagic acid-derived metabolites produced by human colonic microflora, exhibit estrogenic and antiestrogenic activities. J Agric Food Chem 54: 1611-1620, 2006.

271. Larrosa M, González-Sarrías A, Yáñez-Gascón MJ, Selma MV, Azorín-Ortuño M, Toti S, Tomás-Barberán F, Dolara P, and Espín JC. Anti-inflammatory properties of a pomegranate extract and its metabolite urolithin- $\mathrm{A}$ in a colitis rat model and the effect of colon inflammation on phenolic metabolism. J Nutr Biochem 21: 717-725, 2010.

272. Larsson SC, Andersson SO, Johansson JE, and Wolk A. Fruit and vegetable consumption and risk of bladder cancer: a prospective cohort study. Cancer Epidemiol Biomarkers Prev 17: 2519-2522, 2008.

273. Larsson SC, Hakansson N, Naslund I, Bergkvist L, and Wolk A. Fruit and vegetable consumption in relation to pancreatic cancer risk: a prospective study. Cancer Epidemiol Biomarkers Prev 15: 301-305, 2006.

274. Le Bars PL, Velasco FM, Ferguson JM, Dessain EC, Kieser $\mathrm{M}$, and Hoerr R. Influence of the severity of cognitive impairment on the effect of the Ginkgo biloba extract EGb 761 in Alzheimer's disease. Neuropsychobiology 45: 19-26, 2002.

275. Lekakis J, Rallidis LS, Andreadou I, Vamvakou G, Kazantzoglou G, Magiatis P, Skaltsounis AL, and Kremastinos DT. Polyphenolic compounds from red grapes acutely improve endothelial function in patients with coronary heart disease. Eur J Cardiovasc Prev Rehabil 12: 596-600, 2005.

276. Letenneur L, Proust-Lima C, Le GA, Dartigues JF, and Barberger-Gateau P. Flavonoid intake and cognitive decline over a 10-year period. Am J Epidemiol 165: 1364-1371, 2007.

277. Li C, Lee MJ, Sheng S, Meng X, Prabhu S, Winnik B, Huang B, Chung JY, Yan S, Ho CT, and Yang CS. Structural identification of two metabolites of catechins and their kinetics in human urine and blood after tea ingestion. Chem Res Toxicol 13: 177-184, 2000.

278. Li N, Sun Z, Han C, and Chen J. The chemopreventive effects of tea on human oral precancerous mucosa lesions. Proc Soc Exp Biol Med 220: 218-224, 1999.

279. Li Y, Ross-Viola JS, Shay NF, Moore DD, and Ricketts ML. Human CYP3A4 and murine Cyp3A11 are regulated by equol and genistein via the pregnane $X$ receptor in a species-specific manner. J Nutr 139: 898-904, 2009.

280. Lin HH, Chen JH, Huang CC, and Wang CJ. Apoptotic effect of 3,4-dihydroxybenzoic acid on human gastric carcinoma cells involving JNK/p38 MAPK signalling activation. Int J Cancer 120: 2306-2316, 2007.

281. Lippi G, Franchini M, Favaloro EJ, and Targher G. Moderate red wine consumption and cardiovascular disease risk: beyond the "French paradox". Semin Thromb Hemost 36: 59-70, 2010.

282. Lodi F, Jimenez R, Moreno L, Kroon PA, Needs PW, Hughes DA, Santos-Buelga C, González-Paramas A, Cogolludo A, Lopez-Sepulveda R, Duarte J, and Perez-Vizcaino F. Glucuronidated and sulfated metabolites of the flavonoid quercetin prevent endothelial dysfunction but lack direct vasorelaxant effects in rat aorta. Atherosclerosis 204: 34-39, 2009.

283. Loke WM, Proudfoot JM, McKinley AJ, Needs PW, Kroon PA, Hodgson JM, and Croft KD. Quercetin and its in vivo metabolites inhibit neutrophil-mediated low-density lipoprotein oxidation. J Agric Food Chem 56: 3609-3615, 2008.

284. Lorenz M, Jochmann N, von Krosigk A, Martus P, Baumann G, Stangl K, and Stangl V. Addition of milk prevents vascular protective effects of tea. Eur Heart J 28: 219-223, 2007.

285. Lotito SB, Zhang WJ, Yang CS, Crozier A, and Frei B. Metabolic conversion of dietary flavonoids alters their antiinflammatory and antioxidant properties. Free Radic Biol Med 51: 454-463, 2011.

286. Lu H, Meng X, Li C, Sang S, Patten C, Sheng S, Hong J, Bai N, Winnik B, Ho CT, and Yang CS. Glucuronides of tea 
catechins: enzymology of biosynthesis and biological activities. Drug Metab Dispos 31: 452-461, 2003.

287. Luo H, Tang L, Tang M, Billam M, Huang T, Yu J, Wei Z, Liang Y, Wang K, Zhang ZQ, Zhang L, and Wang JS. Phase IIa chemoprevention trial of green tea polyphenols in highrisk individuals of liver cancer: modulation of urinary excretion of green tea polyphenols and 8-hydroxydeoxyguanosine. Carcinogenesis 27: 262-268, 2006.

288. Ma Y, Njike VY, Millet J, Dutta S, Doughty K, Treu JA, and Katz DL. Effects of walnut consumption on endothelial function in type 2 diabetic subjects: a randomized controlled crossover trial. Diabetes Care 33: 227-232, 2010.

289. Macready AL, Kennedy OB, Ellis JA, Williams CM, Spencer JP, and Butler LT. Flavonoids and cognitive function: a review of human randomized controlled trial studies and recommendations for future studies. Genes Nutr 4: 227-242, 2009.

290. Maher P, Akaishi T, and Abe K. Flavonoid fisetin promotes ERK-dependent long-term potentiation and enhances memory. Proc Natl Acad Sci U S A 103: 16568-16573, 2006.

291. Manach C, Williamson G, Morand C, Scalbert A, and Rémésy C. Bioavailability and bioefficacy of polyphenols in humans. I. Review of 97 bioavailability studies. Am J Clin Nutr 81: 2230S-2242S, 2005.

292. Mansvelt EP, van Velden DP, Fourie E, Rossouw M, van Rensburg SJ, and Smuts CM. The in vivo antithrombotic effect of wine consumption on human blood platelets and hemostatic factors. Ann N Y Acad Sci 957: 329-332, 2002.

293. Manzano S and Williamson G. Polyphenols and phenolic acids from strawberry and apple decrease glucose uptake and transport by human intestinal Caco-2 cells. Mol Nutr Food Res 54: 1773-1780, 2010.

294. Marks SC, Mullen W, Borges G, and Crozier A. Absorption, metabolism, and excretion of cider dihydrochalcones in healthy humans and subjects with an ileostomy. J Agric Food Chem 57: 2009-2015, 2009.

295. Matsumoto H, Nakamura Y, Iida H, Ito K, and Ohguro H. Comparative assessment of distribution of blackcurrant anthocyanins in rabbit and rat ocular tissues. Exp Eye Res 83: 348-356, 2006.

296. McAnlis GT, McEneny J, Pearce J, and Young IS. Black tea consumption does not protect low density lipoprotein from oxidative modification. Eur J Clin Nutr 52: 202-206, 1998.

297. McAnulty SR, McAnulty LS, Morrow JD, Khardouni D, Shooter L, Monk J, Gross S, and Brown V. Effect of daily fruit ingestion on angiotensin converting enzyme activity, blood pressure, and oxidative stress in chronic smokers. Free Radic Res 39: 1241-1248, 2005.

298. McDonald MS, Hughes M, Lean MEJ, Burns J, Matthews D, and Crozier A. Survey of the free and conjugated flavonol content of sixty five red wines of different geographical origins. J Agric Food Chem 46: 368-375, 1998.

299. McGhie TK, Aingie GD, Barnet LE, Cooney JM, and Jensen DJ. Anthocyanin glycosides from berry fruit are absorbed and excreted unmetabolised by both human and rats. $J$ Agric Food Chem 51: 4539-4548, 2003.

300. McKay DL, Chen CY, Saltzman E, and Blumberg JB. Hibiscus sabdariffa L. tea (tisane) lowers blood pressure in prehypertensive and mildly hypertensive adults. J Nutr 140: 298-303, 2010.

301. McMullen MK, Whitehouse JM, Shine G, and Towell A. Habitual coffee and tea drinkers experienced increases in blood pressure after consuming low to moderate doses of caffeine; these increases were larger upright than in the supine posture. Food Funct 2: 197-203, 2011.
302. Mellen PB, Daniel KR, Brosnihan KB, Hansen KJ, and Herrington DM. Effect of muscadine grape seed supplementation on vascular function in subjects with or at risk for cardiovascular disease: a randomized crossover trial. J Am Coll Nutr 29: 469-475, 2010.

303. Meng X, Sang S, Zhu N, Lu H, Sheng S, Lee MJ, Ho CT, and Yang CS. Identification and characterization of methylated and ring-fission metabolites of tea catechins formed in humans, mice, and rats. Chem Res Toxicol 15: 1042-1050, 2002.

304. Mercanligil SM, Arslan P, Alasalvar C, Okut E, Akgul E, Pinar A, Geyik PO, Tokgozoglu L, and Shahidi F. Effects of hazelnut-enriched diet on plasma cholesterol and lipoprotein profiles in hypercholesterolemic adult men. Eur J Clin Nutr 61: 212-220, 2007.

305. Mesas AE, Leon-Munoz LM, Rodriguez-Artalejo F, and Lopez-Garcia E. The effect of coffee on blood pressure and cardiovascular disease in hypertensive individuals: a systematic review and meta-analysis. Am J Clin Nutr 94: 11131126, 2011.

306. Middleton E, Jr., Kandaswami C, and Theoharides TC. The effects of plant flavonoids on mammalian cells: implications for inflammation, heart disease, and cancer. Pharmacol Rev 52: 673-751, 2000.

307. Miene C, Weise A, and Glei M. Impact of polyphenol metabolites produced by colonic microbiota on expression of COX-2 and GSTT2 in human colon cells (LT97). Nutr Cancer 63: 653-662, 2011

308. Milder IEJ, Kuijsteen A, Arts IC, Feskens EJ, Kampman E, Hollman PC, and Van 't Veer P. Relation between plasma enterodiol and enterolactone and dietary intake of lignans in a Dutch endoscopy-based population. J Nutr 137: 12661271, 2007.

309. Mink PJ, Scrafford CG, Barraj LM, Harnack L, Hong CP, Nettleton JA, and Jacobs DR, Jr. Flavonoid intake and cardiovascular disease mortality: a prospective study in postmenopausal women. Am J Clin Nutr 85: 895-909, 2007.

310. Mix JA and Crews WD, Jr. An examination of the efficacy of Ginkgo biloba extract EGb761 on the neuropsychologic functioning of cognitively intact older adults. J Altern Complement Med 6: 219-229, 2000.

311. Mohan M, Waghulde H, and Kasture S. Effect of pomegranate juice on Angiotensin II-induced hypertension in diabetic Wistar rats. Phytother Res 24 Suppl 2: S196-S203, 2010.

312. Moller P, Loft S, Alfthan G, and Freese R. Oxidative DNA damage in circulating mononuclear blood cells after ingestion of blackcurrant juice or anthocyanin-rich drink. Mutat Res 551: 119-126, 2004.

313. Moller P, Vogel U, Pedersen A, Dragsted LO, Sandstrom B, and Loft $S$. No effect of 600 grams fruit and vegetables per day on oxidative DNA damage and repair in healthy nonsmokers. Cancer Epidemiol 12: 1016-1022, 2003.

314. Monge P, Solheim E, and Scheline RR. Dihydrochalcone metabolism in the rat: phloretin. Xenobiotica 14: 917-924, 1984.

315. Morand C, Dubray C, Milenkovic D, Lioger D, Martin JF, Scalbert A, and Mazur A. Hesperidin contributes to the vascular protective effects of orange juice: a randomized crossover study in healthy volunteers. Am J Clin Nutr 93: 73-80, 2011.

316. Mukuddem-Petersen J, Oosthuizen W, and Jerling JC. A systematic review of the effects of nuts on blood lipid profiles in humans. J Nutr 135: 2082-2089, 2005. 
317. Mulder T, van Platerink CJ, Wijnand Schuyl PJ, and van Amelsvoort JM. Analysis of theaflavins in biological fluids using liquid chromatography-electrospray mass spectrometry. J Chromatogr B 760: 271-279, 2001.

318. Mulder TP, Rietveld AG, and van Amelsvoort JM. Consumption of both black tea and green tea results in an increase in the excretion of hippuric acid into urine. Am J Clin Nutr 81: 256S-260S, 2005.

319. Mullen W, Archeveque M-A, Edwards CA, and Crozier A. Bioavailability and metabolism of orange juice flavanones in humans: impact of a full fat yogurt. J Agric Food Chem 56: 11157-11164, 2008.

320. Mullen W, Borges G, Donovan JL, Edwards CA, Serafini M, Lean MEJ, and Crozier A. Milk decreases urinary excretion but not plasma pharmacokinetics of cocoa flavan-3-ol metabolites in humans. Am J Clin Nut 89: 1784-1791, 2009.

321. Mullen W, Edwards CA, and Crozier, A. Absorption, excretion and metabolic profiling of methyl-, glucuronyl-, glucosyl and sulpho-conjugates of quercetin in human plasma and urine after ingestion of onions. Br J Nutr 96: 107-116, 2006.

322. Mullen W, Edwards CA, Serafini M, and Crozier A. Bioavailability of pelargonidin-3-O-glucoside and its metabolites in humans following the ingestion of strawberries with and without cream. J Agric Food Chem 56: 713-719, 2008.

323. Mullen W, Rouanet J-M, Auger C, Teissedre P-L, Caldwell ST, Hartley RC, Lean MEJ, Edwards CA, and Crozier A. Bioavailability of $\left[2-{ }^{14} \mathrm{C}\right]$ quercetin- $4{ }^{\prime}$-glucoside in rats. $J$ Agric Food Chem 56: 12127-12137, 2008.

324. Mullen W, McGinn J, Lean MEJ, MacLean MR, Gardner P, Duthie GG, and Crozier A. (2002). Ellagitannins, flavonoids and other phenolics in red raspberries and their contribution to antioxidant capacity and vasorelaxation properties. $J$ Agric Food Chem 50: 5191-5196, 2002.

325. Muniyappa R, Hall G, Kolodziej TL, Karne RJ, Crandon SK, and Quon MJ. Cocoa consumption for 2 wk enhances insulin-mediated vasodilatation without improving blood pressure or insulin resistance in essential hypertension. Am J Clin Nutr 88: 1685-1696, 2008.

326. Murphy KJ, Chronopoulos AK, Singh I, Francis MA, Moriarty H, Pike MJ, Turner AH, Mann NJ, and Sinclair AJ. Dietary flavanols and procyanidin oligomers from cocoa (Theobroma cacao) inhibit platelet function. Am J Clin Nutr 77: 1466-1473, 2003.

327. Nagahama $Y$, Nabatame $H$, Okina T, Yamauchi $H$, Narita M, Fujimoto N, Murakami M, Fukuyama H, and Matsuda $\mathrm{M}$. Cerebral correlates of the progression rate of the cognitive decline in probable Alzheimer's disease. Eur Neurol 50: 1-9, 2003.

328. Naissides M, Mamo JC, James AP, and Pal S. The effect of chronic consumption of red wine on cardiovascular disease risk factors in postmenopausal women. Atherosclerosis 185: 438-445, 2006.

329. Naissides M, Pal S, Mamo JC, James AP, and Dhaliwal S. The effect of chronic consumption of red wine polyphenols on vascular function in postmenopausal women. Eur J Clin Nutr 60: 740-745, 2006.

330. Napoli R, Cozzolino D, Guardasole V, Angelini V, Zarra E, Matarazzo M, Cittadini A, Sacca L, and Torella R. Red wine consumption improves insulin resistance but not endothelial function in type 2 diabetic patients. Metab Clin Exper 54: 306-313, 2005.

331. Naruszewicz M, Laniewska I, Millo B, and Dluzniewski M. Combination therapy of statin with flavonoids rich extract from chokeberry fruits enhanced reduction in cardiovascular risk markers in patients after myocardial infraction (MI). Atherosclerosis 194: e179-e184, 2007.

332. Natella F, Nardini M, Belelli F, Pignatelli P, Di Santo S, Ghiselli A, Violi F, and Scaccini C. Effect of coffee drinking on platelets: inhibition of aggregation and phenols incorporation. Br J Nutr 100: 1276-1282, 2008.

333. Njike VY, Faridi Z, Shuval K, Dutta S, Kay CD, West SG, Kris-Etherton PM, and Katz DL. Effects of sugar-sweetened and sugar-free cocoa on endothelial function in overweight adults. Int J Cardiol 149: 83-88, 2011.

334. Noordzij M, Uiterwaal CS, Arends LR, Kok FJ, Grobbee DE, and Geleijnse JM. Blood pressure response to chronic intake of coffee and caffeine: a meta-analysis of randomized controlled trials J Hypertens 23: 921-928, 2005.

335. Nurk E, Refsum H, Drevon CA, Tell GS, Nygaard HA, Engedal $\mathrm{K}$, and Smith AD. Intake of flavonoid-rich wine, tea, and chocolate by elderly men and women is associated with better cognitive test performance. J Nutr 139: 120-127, 2009.

336. Ottaviani JL, Momma TY, Heiss C, Kwik-Uribe C, Schroeter $\mathrm{H}$, and Keen $\mathrm{CL}$. The stereochemical configuration of flavonols influences the level and metabolism of flavonols in humans and their biological activity in vivo. Free Radic Biol Med 50: 237-244, 2011.

337. Ottaviani JI, Momma TY, Kuhnle GK, Keen CL, and Schroeter H. Structurally related (-)-epicatechin metabolites in humans: assessment using de novo chemically synthesized authentic standards. Free Radic Biol Med 52: 1403-1412, 2012.

338. Ozeki Y, Matsuba Y, Abe Y, Umemoto N, and Sasaki N. Pigment biosynthesis I. Anthocyanins. In: Plant Metabolism and Biotechnology, edited by Ashihara H, Crozier A, and Komamine A. Chichester, United Kingdom: Wiley, 2011, pp. $155-181$.

339. Pace-Asciak CR, Rounova O, Hahn SE, Diamandis EP, and Goldberg DM. Wines and grape juices as modulators of platelet aggregation in healthy human subjects. Clin Chim Acta 246: 163-182, 1996.

340. Paixão J, Dinis TC, and Almeida LM. Dietary anthocyanins protect endothelial cells against peroxynitrite-induced mitochondrial apoptosis pathway and Bax nuclear translocation: an in vitro approach. Apoptosis 16: 976-989, 2011.

341. Pan Y, Anthony M, and Clarkson TB. Effect of estradiol and soy phytoestrogens on choline acetyltransferase and nerve growth factor mRNAs in the frontal cortex and hippocampus of female rats. Proc Soc Exp Biol Med 221: 118-125, 1999.

342. Pan Y, Anthony M, and Clarkson TB. Evidence for upregulation of brain-derived neurotrophic factor mRNA by soy phytoestrogens in the frontal cortex of retired breeder female rats. Neurosci Lett 261: 17-20, 1999.

343. Papamichael CM, Aznaouridis KA, Karatzis EN, Karatzi KN, Stamatelopoulos KS, Vamvakou G, Lekakis JP, and Mavrikakis ME. Effect of coffee on endothelial function in healthy subjects: the role of caffeine. Clin Sci 109: 55-60, 2005.

344. Park CS, Kim W, Woo JS, Ha SJ, Kang WY, Hwang SH, Park YW, Kim YS, Ahn YK, and Jeong MH. Green tea consumption improves endothelial function but not circulating endothelial progenitor cells in patients with chronic renal failure. Int J Cardiol 145: 261-262, 2010.

345. Park YK, Kim JS, and Kang MH. Concord grape juice supplementation reduces blood pressure in Korean hypertensive men: double-blind, placebo controlled intervention trial. BioFactors 22: 145-147, 2004. 
346. Pashikanti S, de Alba DR, Boissonneault GA, and Cervantes-Laurean D. Rutin metabolites: novel inhibitors of nonoxidative advanced glycation end products. Free Radic Biol Med 48: 656-663, 2010.

347. Pasquale EB. Eph-ephrin bidirectional signaling in physiology and disease. Cell 133: 38-52, 2008.

348. Patel KR, Brown VA, Jones DJ, Britton RG, Hemingway D, Miller AS, West KP, Booth TD, Perloff M, Crowell JA, Brenner DE, Steward WP, Gescher AJ, and Brown K. Clinical pharmacology of resveratrol and Its metabolites in colorectal cancer patients. Cancer Res 70: 7392-7399, 2010.

349. Pavlica S and Gebhardt R. Protective effects of flavonoids and two metabolites against oxidative stress in neuronal PC12 cells. Life Sci 86: 79-86, 2010.

350. Pearson DA, Paglieroni TG, Rein D, Wun T, Schramm DD, Wang JF, Holt RR, Gosselin R, Schmitz HH, and Keen CL. The effects of flavanol-rich cocoa and aspirin on ex vivo platelet function. Thromb Res 106: 191-197, 2002.

351. Pedersen A, Johansen C, and Grønbaek M. Relations between amount and type of alcohol and colon and rectal cancer in a Danish population based cohort study. Gut 52: 861-867, 2003.

352. Pellegrini N, Pareti FI, Stabile F, Brusamolino A, and Simonetti P. Effects of moderate consumption of red wine on platelet aggregation and haemostatic variables in healthy volunteers. Eur J Clin Nutr 50: 209-213, 1996.

353. Peñalvo JL, Haajanen KM, Botting N, and Adlercreutz H. Quantification of lignans in food using isotope dilution gas chromatography/mass spectrometry. J Agric Food Chem 53: 9342-9347, 2005.

354. Peñalvo JL, Heinonen S-M, Aura A-M, and Adlercreutz H. Dietary sesamin is converted to enterolactone in humans. $J$ Nutr 135: 1056-1062, 2005.

355. Pipingas A, Silberstein RB, Vitetta L, Rooy CV, Harris EV, Young JM, Frampton CM, Sali A, and Nastasi J. Improved cognitive performance after dietary supplementation with a Pinus radiata bark extract formulation. Phytother Res 22: 1168-1174, 2008

356. Plaumann B, Fritsche M, Rimpler H, Brandner G, and Hess RD. Flavonoids activate wild-type p53. Oncogene 13: 16051614, 1996.

357. Polagruto JA, Gross HB, Kamangar F, Kosuna K, Sun B, Fujii H, Keen CL, and Hackman RM. Platelet reactivity in male smokers following the acute consumption of a flavanol-rich grape seed extract. J Med Food 10: 725-730, 2007.

358. Poquet L, Clifford MN, and Williamson G. Effect of dihydrocaffeic acid on UV irradiation of human keratinocyte HaCaT cells. Arch Biochem Biophys 476: 196-204, 2008.

359. Poulose SM and Shukitt-Hale B. Effects of flavonoids on cognitive performance. In: Flavonoids and Related Compounds: Bioavailability and Function, edited by Spencer JPE and Crozier A. Oxidative Stress and Disease, Vol. 30, edited by Packer L and Cadenas H. Boca Raton, FL: CRC Press, 2012, pp. 393-411.

360. Princen HM, van Duyvenvoorde W, Buytenhek R, Blonk C, Tijburg LB, Langius JA, Meinders AE, and Pijl H. No effect of consumption of green and black tea on plasma lipid and antioxidant levels and on LDL oxidation in smokers. Arterioscler Thromb Vasc Biol 18: 833-841, 1998.

361. Prior RL and Wu X. Anthocyanins: structural characteristics that result in unique metabolic patterns and biological activities. Free Radic Res 40: 1014-1028, 2006.

362. Proteggente AR, Basu-Modak S, Kuhnle G, Gordon MJ, Youdim K, Tyrrell R, and Rice-Evans CA. Hesperetin glu- curonide, a photoprotective agent arising from flavonoid metabolism in human skin fibroblasts. Photochem Photobiol 78: 256-261, 2003.

363. Pu F, Mishima K, Irie K, Motohashi K, Tanaka Y, Orito K, Egawa T, Kitamura Y, Egashira N, Iwasaki K, and Fujiwara M. Neuroprotective effects of quercetin and rutin on spatial memory impairment in an 8-arm radial maze task and neuronal death induced by repeated cerebral ischemia in rats. J Pharmacol Sci 104: 329-334, 2007.

364. Puddey IB, Beilin LJ, and Vandongen R. Regular alcohol use raises blood pressure in treated hypertensive subjects. A randomised controlled trial. Lancet 1: 647-651, 1987.

365. Puddey IB, Beilin LJ, Vandongen R, Rouse IL, and Rogers $P$. Evidence for a direct effect of alcohol consumption on blood pressure in normotensive men. A randomized controlled trial. Hypertension 7: 707-713, 1985.

366. Qin Y, Xia M, Ma J, Hao Y, Liu J, Mou H, Cao L, and Ling W. Anthocyanin supplementation improves serum LDLand HDL-cholesterol concentrations associated with the inhibition of cholesteryl ester transfer protein in dyslipidemic subjects. Am J Clin Nutr 90: 485-492, 2009.

367. Radnai B, Tucsek Z, Bognar Z, Antus C, Mark L, Berente Z, Gallyas F, Jr., Sumegi B, and Veres B. Ferulaldehyde, a water-soluble degradation product of polyphenols, inhibits the lipopolysaccharide-induced inflammatory response in mice. J Nutr 139: 291-297, 2009.

368. Ramirez MR, Izquierdo I, do Carmo Bassols Raseira M, Zuanazzi JA, Barros D, and Henriques AT. Effect of lyophilised Vaccinium berries on memory, anxiety and locomotion in adult rats. Pharmacol Res 52: 457-462, 2005.

369. Ras RT, Zock PL, and Draijer R. Tea consumption enhances endothelial-dependent vasodilation; a meta-analysis. PLoS One 6: e16974, 2011.

370. Rechner AR and Kroner C. Anthocyanins and colonic metabolites of dietary polyphenols inhibit platelet function. Thromb Res 116: 327-334, 2005.

371. Rechner AR, Smith MA, Kuhnle G, Gibson GR, Debnam ES, Srai SK, Moore KP, and Rice-Evans CA. Colonic metabolism of dietary polyphenols: influence of structure on microbial fermentation products. Free Radic Biol Med 36: 212-225, 2004.

372. Rein D, Lotito S, Holt RR, Keen CL, Schmitz HH, and Fraga CG. Epicatechin in plasma: in vivo determination and effect of chocolate consumption on plasma oxidative status. J Nutr 130: 2109S-2114S, 2000.

373. Rein D, Paglieroni TG, Wun T, Pearson DA, Schmitz HH, Gosselin R, and Keen CL. Cocoa inhibits platelet activation and function. Am J Clin Nutr 72: 30-35, 2000.

374. Reshef N, Hayari Y, Goren C, Boaz M, Madar Z, and Knobler H. Antihypertensive effect of sweetie fruit in patients with stage I hypertension. Am J Hypertens 18: 13601363, 2005.

375. Riboli E and Norat T. Epidemiologic evidence of the protective effect of fruit and vegetables on cancer risk. Am J Clin Nutr 78: 559S-569S, 2003.

376. Richelle M, Tavazzi I, Enslen M, and Offord EA. Plasma kinetics in man of epicatechin from black chocolate. Eur J Clin Nutr 53: 22-26, 1999.

377. Richling E. Bioavailability of dihydrochalcones. In: Flavonoids and Related Compounds: Bioavailability and Function, edited by Spencer JPE and Crozier A. Oxidative Stress and Disease, Vol. 30, edited by Packer L and Cadenas H. Boca Raton, FL: CRC Press, 2012, pp. 157-165. 
378. Rifler JP, Lorcerie F, Durand P, Delmas D, Ragot K, Limagne E, Mazue F, Riedinger JM, d'Athis P, Hudelot B, Prost M, Lizard G, and Latruffe N. A moderate red wine intake improves blood lipid parameters and erythrocytes membrane fluidity in post myocardial infarct patients. Mol Nutr Food Res 56: 345-351, 2012.

379. Rimbaud S, Ruiz M, Piquereau J, Mateo P, Fortin D, Veksler V, Garnier A, and Ventura-Clapier R. Resveratrol improves survival, hemodynamics and energetics in a rat model of hypertension leading to heart failure. PLoS One 6: e26391, 2011.

380. Rios LY, Bennett RN, Lazarus SA, Rémésy C, Scalbert A, and Williamson G. Cocoa procyanidins are stable during gastric transit in humans. Am J Clin Nutr 76: 1106-1110, 2002.

381. Riso P, Visioli F, Gardana C, Grande S, Brusamolino A, Galvano F, Galvano G, and Porrini M. Effects of blood orange juice intake on antioxidant bioavailability and on different markers related to oxidative stress. J Agric Food Chem 53: 941-947, 2005.

382. Rizza S, Muniyappa R, Iantorno M, Kim JA, Chen $H$, Pullikotil P, Senese N, Tesauro M, Lauro D, Cardillo C, and Quon MJ. Citrus polyphenol hesperidin stimulates production of nitric oxide in endothelial cells while improving endothelial function and reducing inflammatory markers in patients with metabolic syndrome. J Clin Endocrinol Metab 96: E782- E792, 2011.

383. Rolls ET and Kesner RP. A computational theory of hippocampal function, and empirical tests of the theory. Prog Neurobiol 79: 1-48, 2006.

384. Ronksley PE, Brien SE, Turner BJ, Mukamal KJ, and Ghali WA. Association of alcohol consumption with selected cardiovascular disease outcomes: a systematic review and meta-analysis. BMJ 342: d671, 2011.

385. Roomi MW, Ivanov V, Kalinovsky T, Niedzwiecki A, and Rath M. Anticancer effect of lysine, proline, arginine, ascorbic acid and green tea extract on human renal adenocarcinoma line 786-0. Oncol Rep 16: 943-947, 2006.

386. Roowi S, Mullen W, Edwards CA, and Crozier A. Yoghurt impacts on the excretion of phenolic acids derived from colonic breakdown of orange juice flavanones in humans. Mol Nutr Food Res 53: S68-S75, 2009.

387. Roowi S, Stalmach A, Mullen W, Lean MEJ, Edwards CA, and Crozier A. Green tea flavan-3-ols: colonic degradation and urinary excretion of catabolites by humans. J Agric Food Chem 58: 1296-1304, 2010.

388. Ros E, Nunez I, Perez-Heras A, Serra M, Gilabert R, Casals E, and Deulofeu R. A walnut diet improves endothelial function in hypercholesterolemic subjects: a randomized crossover trial. Circulation 109: 1609-1614, 2004

389. Rosenblat M, Volkova N, Coleman R, and Aviram M. Pomegranate byproduct administration to apolipoprotein edeficient mice attenuates atherosclerosis development as a result of decreased macrophage oxidative stress and reduced cellular uptake of oxidized low-density lipoprotein. $J$ Agric Food Chem 54: 1928-1935, 2006.

390. Roura E, Almajano MP, Mata-Bilbao ML, Andrés-Lacueva C, Estruch R, and Lamuela-Raventós RM. Human urine: epicatechin metabolites and antioxidant activity after cocoa intake. Free Radic Res 41: 943-949, 2007.

391. Roura E, Andés-Lacueva C, Estruch R, Mata-Bilbao ML, Izquierdo-Pulido $\mathrm{M}$, Waterhouse $\mathrm{AL}$, and Lamuela-Raventós RM. Milk does not affect the bioavailability of cocoa powder flavonoid in healthy human. Ann Nutr Metab 51: 493-498, 2007.
392. Roura E, Andrés-Lacueva C, Estruch R, Lourdes Mata Bilbao M, Izquierdo-Pulido M, and Lamuela-Raventós RM. The effects of milk as a food matrix for polyphenols on the excretion profile of cocoa (-)-epicatechin metabolites in healthy human subjects. Br J Nutr 100: 846-851, 2008.

393. Roura E, Andrés-Lacueva C, Jáuregui O, Badia E, Estruch R, Izquierdo-Pulido M, and Lamuela-Raventós RM. Rapid liquid chromatography tandem mass spectrometer assay to quantify plasma (-)-epicatechin metabolites after ingestion of a standard portion of cocoa beverage in humans. J Agric Food Chem 53: 6190-6194, 2005.

394. Rowlands DJ, Chapple S, Siow RC, and Mann GE. Equolstimulated mitochondrial reactive oxygen species activate endothelial nitric oxide synthase and redox signaling in endothelial cells: roles for F-actin and GPR30. Hypertension 57: 833-840, 2011.

395. Ruitenberg A, den Heijer T, Bakker SL, van Swieten JC, Koudstaal PJ, Hofman A, and Breteler MM. Cerebral hypoperfusion and clinical onset of dementia: the Rotterdam Study. Ann Neurol 57: 789-794, 2005.

396. Russell WR, Labat A, Scobbie L, and Duncan SH. Availability of blueberry phenolics for microbial metabolism in the colon and the potential inflammatory implications. Mol Nutr Food Res 51: 726-731, 2007.

397. Ryan J, Croft K, Mori T, Wesnes K, Spong J, Downey L, Kure C, Lloyd J, and Stough C. An examination of the effects of the antioxidant Pycnogenol on cognitive performance, serum lipid profile, endocrinological and oxidative stress biomarkers in an elderly population. J Psychopharmacol 22: 553-562, 2008.

398. Sakakibara H, Ogawa T, Koyanagi A, Kobayashi S, Goda T, Kumazawa S, Kobayashi H, and Shimoi K. Distribution and excretion of bilberry anthocyanins in mice. J Agric Food Chem 57: 7681-7686, 2009.

399. Sakata Y, Zhuang H, Kwansa H, Koehler RC, and Doré S. Resveratrol protects against experimental stroke: putative neuroprotective role of heme oxygenase 1. Exp Neurol 224: 325-329, 2010.

400. Sang S, Lee MJ, Yang I, Buckley B, and Yang CS. Human urinary metabolite profile of tea polyphenols analyzed by liquid chromatography/electrospray ionization tandem mass spectrometry with data-dependent acquisition. Rapid Comm Mass Spectrom 22: 1567-1578, 2008.

401. Sari I, Baltaci Y, Bagci C, Davutoglu V, Erel O, Celik H, Ozer O, Aksoy N, and Aksoy M. Effect of pistachio diet on lipid parameters, endothelial function, inflammation, and oxidative status: a prospective study. Nutrition 26: 399-404, 2010.

402. Sarwar N, Thompson AJ, and Di Angelantonio E. Markers of inflammation and risk of coronary heart disease. Dis Markers 26: 217-225, 2009.

403. Scholey AB, French SJ, Morris PJ, Kennedy DO, Milne AL, and Haskell CF. Consumption of cocoa flavanols results in acute improvements in mood and cognitive performance during sustained mental effort. J Psychopharmacol 24: 15051514,2010

404. Schroeter H, Heiss C, Balzer J, Kleinbongard P, Keen CL, Hollenberg NK, Sies H, Kwik-Uribe C, Schmitz HH, and Kelm M. (-)-Epicatechin mediates beneficial effects of flavanol-rich cocoa on vascular function in humans. Proc Natl Acad Sci U S A 103: 1024-1029, 2006.

405. Science Daily. Red wine compound resveratrol demonstrates significant health effects. Available at www.sciencedaily.com/releases/2009/06/090611174052.htm, 2009. 
406. Seeram NP, Aronson WJ, Zhang Y, Henning SM, Moro A, Lee RP, Sartippour M, Harris DM, Rettig M, Suchard MA, Pantuck AJ, Belldegrun A, and Heber D. Pomegranate ellagitannin-derived metabolites inhibit prostate cancer growth and localize to the mouse prostate gland. J Agric Food Chem 55: 7732-7737, 2007.

407. Seeram NP, Henning SM, Zhang Y, Suchard M, Li Z, and Heber D. Pomegranate juice ellagitannin metabolites are present in human plasma and some persist in urine for up to 48 hours. J Nutr 136: 2481-2485, 2006.

408. Self HL, Brown RR, and Price JM. Quantitative studies on the metabolites of tryptophan in the urine of swine. J Nutr 70: $21-25,1960$.

409. Setchell KD, Brown NM, and Lydeking-Olsen E. The clinical importance of the metabolite equol-a clue to the effectiveness of soy and its isoflavones. J Nutr 132: 3577-3584, 2002.

410. Setchell KD, Brown NM, Zimmer-Nechemias L. Brashear WT, Wolfe BE, Kirschner AS, and Heubi JE. Evidence for lack of absorption of soy isoflavone glycosides in humans, supporting the crucial role intestinal metabolism for bioavailability. Am J Clin Nutr 76: 447-453, 2002.

411. Setchell KD, Faughnan MS, Zimmer-Nechemias L, Brown NM, Wolfe BE, Brashear WT, Desai D, Oldfield MF, Botting $\mathrm{NP}$, and Cassidy A. Comparing the pharmacokinetics of daidzein and genistein with the use of ${ }^{13} \mathrm{C}$-labelled tracers in premenopausal women. Am J Clin Nutr 77: 411-419, 2003.

412. Shahrzad S and Bitsch I. Determination of gallic acid and its metabolites in human plasma and urine by high-performance liquid chromatography. J Chromatogr B 705: 8795, 1998.

413. Shahrzad S, Aoyagi K, Winter A, Koyama A, and Bitsch I. Pharmacokinetics of gallic acid and its relative bioavailability from tea in healthy humans. J Nutr 131: 1207-1210, 2001.

414. Sharma M, Li L, Celver J, Killian C, Kovoor A, and Seeram NP. Effects of fruit ellagitannin extracts, ellagic acid, and their colonic metabolite, urolithin A, on Wnt signaling. J Agric Food Chem 58: 3965-3969, 2010.

415. Shaughnessy KS, Boswall IA, Scanlan AP, Gottschall-Pass KT, and Sweeney MI. Diets containing blueberry extract lower blood pressure in spontaneously hypertensive stroke-prone rats. Nutr Res 29: 130-138, 2009.

416. Shif O, Gillette K, Damkaoutis CM, Carrano C, Robbins SJ, and Hoffman JR. Effects of Ginkgo biloba administered after spatial learning on water maze and radial arm maze performance in young adult rats. Pharmacol Biochem Behav 84: 17-25, 2006.

417. Shirai M, Moon JH, Tsushida T, and Terao J. Inhibitory effect of a quercetin metabolite, quercetin 3-O- $\beta$-D-glucuronide, on lipid peroxidation in liposomal membranes. $J$ Agric Food Chem 49: 5602-5608, 2001.

418. Shirai M, Yamanishi R, Moon JH, Murota K, and Terao J. Effect of quercetin and its conjugated metabolite on the hydrogen peroxide-induced intracellular production of reactive oxygen species in mouse fibroblasts. Biosci Biotechnol Biochem 66: 1015-1021, 2002.

419. Shoji T, Masumoto S, Moriichi N, Akiyama H, Kanda T, Ohtake Y, and Goda Y. Apple procyanidin oligomers absorption in rats after oral administration: analysis of procyanidins in plasma using the porter method and highperformance liquid chromatography/tandem mass spectrometry. J Agric Food Chem 54: 884-892, 2006.
420. Shors TJ, Townsend DA, Zhao M, Kozorovitskiy Y, and Gould E. Neurogenesis may relate to some but not all types of hippocampal-dependent learning. Hippocampus 12: 578584, 2002.

421. Shrime MG, Bauer SR, McDonald AC, Chowdhury NH, Coltart CE, and Ding EL. Flavonoid-rich cocoa consumption affects multiple cardiovascular risk factors in a metaanalysis of short-term studies. J Nutr 141: 1982-1988, 2011.

422. Shukitt-Hale B, Carey A, Simon L, Mark DA, and Joseph JA. Effects of Concord grape juice on cognitive and motor deficits in aging. Nutrition 22: 295-302, 2006.

423. Shukitt-Hale B, Cheng V, and Joseph JA. Effects of blackberries on motor and cognitive function in aged rats. Nutr Neurosci 12: 135-140, 2009.

424. Small SA, Chawla MK, Buonocore M, Rapp PR, and Barnes CA. Imaging correlates of brain function in monkeys and rats isolates a hippocampal subregion differentially vulnerable to aging. Proc Natl Acad Sci U S A 101: 7181-7186, 2004.

425. Small SA, Tsai WY, DeLaPaz R, Mayeux R, and Stern Y. Imaging hippocampal function across the human life span: is memory decline normal or not? Ann Neurol 51: 290-295, 2002.

426. Sone T, Kuriyama S, Nakaya N, Hozawa A, Shimazu T, Nomura K, Rikimaru S, and Tsuji I. Randomized controlled trial for an effect of catechin-enriched green tea consumption on adiponectin and cardiovascular disease risk factors. Food Nutr Res 55: 2011; DOI: 10.3402/fnr.v55i0.8326.

427. Spaak J, Merlocco AC, Soleas GJ, Tomlinson G, Morris BL, Picton P, Notarius CF, Chan CT, and Floras JS. Dose-related effects of red wine and alcohol on hemodynamics, sympathetic nerve activity, and arterial diameter. Am J Physiol 294: H605-H612, 2008.

428. Spencer JP. Beyond antioxidants: the cellular and molecular interactions of flavonoids and how these underpin their actions on the brain. Proc Nutr Soc 69: 244-260, 2010.

429. Spencer JP. Flavonoids: modulators of brain function? Br J Nutr 99 E Suppl 1: ES60- ES77, 2008.

430. Spencer JP. The impact of flavonoids on memory: physiological and molecular considerations. Chem Soc Rev 38: 1152-1161, 2009.

431. Spencer JP. The impact of fruit flavonoids on memory and cognition. Br J Nutr 104 Suppl 3: S40-S47, 2010.

432. Spencer JP. The interactions of flavonoids within neuronal signalling pathways. Genes Nutr 2: 257-273, 2007.

433. Spencer JPE and Crozier A (eds.) In: Flavonoids and Related Compounds: Bioavailability and Function. Oxidative Stress and Disease, Vol. 30, edited by Packer L and Cadenas H. Boca Raton, FL: CRC Press, 2012.

434. Stalmach A, Edwards CA, Wightman J, and Crozier, A. Identification of (poly)phenolic compounds in Concord grape juice and their metabolites in human plasma and urine after juice consumption. J Agric Food Chem 59: 95129522, 2011.

435. Stalmach A. Edwards CA, Wightman J, and Crozier A. Gastrointestinal stability and bioavailability of (poly)phenolic compounds following ingestion of Concord grape juice by humans. Mol Nutr Food Res 56: 497-509, 2012.

436. Stalmach A, Mullen W, Barron D, Uchida K, Yokota T, Cavin C, Steiling H, Williamson G, and Crozier A. Metabolite profiling of hydroxycinnamate derivatives in plasma and urine following the ingestion of coffee by humans: identification of biomarkers of coffee consumption. Drug Met Disp 37: 1759-1768, 2009. 
437. Stalmach A, Mullen W, Pecorari M, Serafini M, and Crozier, A. Bioavailability of C-linked dihydrochalcone and flavanone glucosides in humans following ingestion of unfermented and fermented rooibos teas. J Agric Food Chem 57: 7104-7111, 2009.

438. Stalmach A, Mullen W, Steiling H, Williamson G, Lean MEJ, and Crozier A. Absorption, metabolism, and excretion of green tea flavan-3-ols in humans with an ileostomy. Mol Nutr Food Res 54: 323-334, 2010.

439. Stalmach A, Steiling H, Williamson G, and Crozier A. Bioavailability of chlorogenic acids following acute ingestion of coffee by humans with an ileostomy. Arch Biochem Biophys 501: 98-105, 2010.

440. Stalmach A, Troufflard S, Serafini M, and Crozier A. Absorption, metabolism and excretion of Choladi green tea flavan-3-ols by humans. Mol Nutr Food Res 53: S44-S53, 2009.

441. Stangl D and Thuret S. Impact of diet on adult hippocampal neurogenesis. Genes Nutr 4: 271-282, 2009.

442. Steffen Y, Gruber C, Schewe T, and Sies H. Mono-Omethylated flavanols and other flavonoids as inhibitors of endothelial NADPH oxidase. Arch Biochem Biophys 469: 209-219, 2008.

443. Steptoe A, Gibson EL, Vuononvirta R, Hamer M, Wardle J, Rycroft JA, Martin JF, and Erusalimsky JD. The effects of chronic tea intake on platelet activation and inflammation: a double-blind placebo controlled trial. Atherosclerosis 193: 277-282, 2007.

444. Stoupi S, Williamson G, Drynan JW, Barron D, and Clifford $\mathrm{MN}$. A comparison of the in vitro biotransformation of (-)epicatechin and procyanidin B2 by human faecal microbiota. Mol Nutr Food Res 54: 747-759, 2010.

445. Stoupi S, Williamson G, Drynan JW, Barron D, and Clifford MN. Procyanidin B2 catabolism by human fecal microflora: partial characterization of 'dimeric' intermediates. Arch Biochem Biophys 501: 73-78, 2010.

446. Stoupi S, Williamson G, Viton F, Barron D, King LJ, Brown $\mathrm{JE}$, and Clifford MN. In vivo bioavailability, absorption, excretion, and pharmacokinetics of $\left[{ }^{14} \mathrm{C}\right]$ procyanidin $\mathrm{B} 2$ in male rats. Drug Met Disp 38: 287-291, 2010.

447. Strack D and Wray V. Anthocyanins. In: The Flavonoids: Advances in Research Since 1986, edited by Harborne JB. London: Chapman and Hall, 1992, pp. 1-22.

448. Stull AJ, Cash KC, Johnson WD, Champagne CM, and Cefalu WT. Bioactives in blueberries improve insulin sensitivity in obese, insulin-resistant men and women. J Nutr 140: 1764-1768, 2010.

449. Suganuma M, Okabe S, Oniyama M, Tada $\mathrm{Y}$, Ito $H$, and Fujiki $\mathrm{H}$. Wide distribution of $\left[{ }^{3} \mathrm{H}\right](-)$-epigallocatechin gallate, a cancer preventive tea polyphenol, in mouse tissue. Carcinogenesis 19: 1771-1776, 1998.

450. Sumner MD, Elliott-Eller M, Weidner G, Daubenmier JJ, Chew MH, Marlin R, Raisin CJ, and Ornish D. Effects of pomegranate juice consumption on myocardial perfusion in patients with coronary heart disease. Am J Cardiol 96: 810-814, 2005.

451. Suri S, Liu XH, Rayment S, Hughes DA, Kroon PA, Needs PW, Taylor MA, Tribolo S, and Wilson VG. Quercetin and its major metabolites selectively modulate cyclic GMP-dependent relaxations and associated tolerance in pig isolated coronary artery. Br J Pharmacol 159: 566-575, 2010.

452. Takagaki A, Otani S, and Nanjo F. Antioxidative activity of microbial metabolites of (-)-epigallocatechin gallate produced in rat intestines. Biosci Biotechnol Biochem 75: 582-585, 2011.
453. Taubert D, Roesen R, Lehmann C, Jung N, and Schomig E. Effects of low habitual cocoa intake on blood pressure and bioactive nitric oxide: a randomized controlled trial. JAMA 298: 49-60, 2007.

454. Taubert D, Roesen R, and Schomig E. Effect of cocoa and tea intake on blood pressure: a meta-analysis. Arch Intern Med 167: 626-634, 2007.

455. Thompson HJ, Heimendinger J, Gillette C, Sedlacek SM, Haegele A, O'Neill C, and Wolfe P. In vivo investigation of changes in biomarkers of oxidative stress induced by plant food rich diets. J Agric Food Chem 53: 6126-6132, 2005.

456. Thompson HJ, Heimendinger J, Haegele A, Sedlacek SM, Gillette C, O'Neill C, Wolfe P, and Conry C. Effect of increased vegetable and fruit consumption on markers of oxidative cellular damage. Carcinogenesis 20: 2261-2266, 1999.

457. Tognolini M, Giorgio C, Mohamed IH, Barocelli E, Calani L, Reynaud E, Dangles O, Borges G, Crozier A, Brighenti F, and Del Rio D. Perturbation of the EphA2-EphrinA1 system by colonic (poly)phenol catabolites in human prostate cancer cells. J Agric Food Chem 2012 [Epub ahead of print]; DOI: $10.1021 /$ jf205305m.

458. Tomás-Barberán FA, Cienfuegos-Jovellanos E, Marín A, Muguerza B, Gil-Izquierdo A, Cerda B, Zafrilla P, Morillas J, Mulero J, Ibarra A, Pasamar MA, Ramón D, and Espín JC. A new process to develop a cocoa powder with higher flavonoid monomer content and enhanced bioavailability in healthy humans. J Agric Food Chem 55: 3926-3935, 2007.

459. Tribolo S, Lodi F, Connor C, Suri S, Wilson VG, Taylor MA, Needs PW, Kroon PA, and Hughes DA. Comparative effects of quercetin and its predominant human metabolites on adhesion molecule expression in activated human vascular endothelial cells. Atherosclerosis 197: 50-56, 2008.

460. Trock BJ, Hilakivi-Clarke L, and Clarke R. Meta-analysis of soy intake and breast cancer risk. J Natl Cancer Inst 98: 459471, 2006.

461. Trzeciakiewicz A, Habauzit V, Mercier S, Barron D, UrpiSarda M, Manach C, Offord E, and Horcajada MN. Molecular mechanism of hesperetin-7-O-glucuronide, the main circulating metabolite of hesperidin, involved in osteoblast differentiation. J Agric Food Chem 58: 668-675, 2010.

462. Tsang C, Auger C, Mullen W, Bornet A, Rouanet JM, Crozier A, and Teissedre PL. The absorption, metabolism and excretion of flavan-3-ols and procyanidins following the ingestion of a grape seed extract by rats. Br J Nutr 94: 170-181, 2005.

463. Tsao AS, Liu D, Martin J, Tang XM, Lee JJ, El-Naggar AK, Wistuba I, Culotta KS, Mao L, Gillenwater A, Sagesaka YM, Hong WK, and Papadimitrakopoulou V. Phase II randomized, placebo-controlled trial of green tea extract in patients with high-risk oral premalignant lesions. Cancer Prev Res 2: 931-941, 2009.

464. Unno T. Kondo K, Itakura H, and Takeo T. Analysis of (-)epigallocatechin gallate in human serum obtained after ingesting green tea. Biosci Biotechnol Biochem 60: 2066-2068, 1996.

465. Urpi-Sarda M, Monagas M, Khan N, Lamuela-Raventos RM, Santos-Buelga C, Sacanella E, Castell M, Permanyer J, and Andres-Lacueva C. Epicatechin, procyanidins and phenolic microbial metabolites after cocoa intake in humans and rats. Anal Bioanal Chem 394: 1545-1556, 2009.

466. Urpi-Sarda M, Ramiro-Puig E, Romos-Romero S, Llorach R, Castell M, González-Manzano S, Santos-Buelga C, and Andres-Lacueva C. Distribution of epicatechin metabolites 
in lymphoid tissues and testes of young rats with a cocoaenriched diet. Br J Nutr 103: 1393-1397, 2010.

467. Urpi-Sarda M, Zamora-Ros R, Lamuela-Raventos R, Cherubini A, Jauregui O, de la Torre R, Covas MI, Estruch R, Jaeger $\mathrm{W}$, and Andres-Lacueva C. HPLC-tandem mass spectrometric method to characterize resveratrol metabolism in humans. Clin Chem 53, 292-299, 2007.

468. van de Wetering K, Burkon A, Feddema W, Bot A, de Jonge $\mathrm{H}$, Somoza $\mathrm{V}$, and Borst $\mathrm{P}$. Intestinal breast cancer resistance protein $(\mathrm{BCRP}) / \mathrm{Bcrp} 1$ and multidrug resistance protein 3 (MRP3)/Mrp3 are involved in the pharmacokinetics of resveratrol. Mol Pharmacol 7: 876-885, 2009.

469. van de Wiel A and de Lange DW. Cardiovascular risk is more related to drinking pattern than to the type of alcoholic drinks. Neth J Med 66: 467-473, 2008.

470. van Erk MJ, Roepman P, van der Lende TR, Stierum RH, Aarts JM, van Bladeren PJ, and van Ommen B. Integrated assessment by multiple gene expression analysis of quercetin bioactivity on anticancer-related mechanisms in colon cancer cells in vitro. Eur J Nutr 44: 143-156, 2005.

471. van het Hof $\mathrm{KH}$, de Boer HS, Wiseman SA, Lien N, Westrate JA, and Tijburg LB. Consumption of green or black tea does not increase resistance of low-density lipoprotein to oxidation in humans. Am J Clin Nutr 66: 1125-1132, 1997.

472. van Mierlo LA, Zock PL, van der Knaap HC, and Draijer R. Grape polyphenols do not affect vascular function in healthy men. J Nutr 140: 1769-1773, 2010.

473. van Praag H, Lucero MJ, Yeo GW, Stecker K, Heivand N, Zhao C, Yip E, Afanador M, Schroeter H, Hammerstone J, and Gage FH. Plant-derived flavanol (-)-epicatechin enhances angiogenesis and retention of spatial memory in mice. J Neurosci 27: 5869-5878, 2007.

474. Vastrano BC, Chen Y, Zhu N, Ho CT, Zhou Z, and Rosen RT. Isolation and identification of stilbenes in two varieties of Polygonum cuspidatum. J Agric Food Chem 48: 253-256, 2000.

475. Vaz-da-Silva M, Loureiro AI, Falcao A, Nunes T, Rocha JF, Fernandes-Lopes C, Soares E, Wright L, Almeida L, and Soares-da-Silva P. Effect of food on the pharmacokinetic profile of trans-resveratrol. Int J Clin Pharmacol Ther 46: 564570, 2008.

476. Verzelloni E, Pellacani C, Tagliazucchi D, Tagliaferri S, Calani L, Costa LG, Brighenti F, Borges G, Crozier A, Conte A, and Del Rio D. Antiglycative and neuroprotective activity of colon-derived polyphenol catabolites. Mol Nutr Food Res 55 Suppl 1: S35-S43, 2011.

477. Verzelloni E, Tagliazucchi D, Del Rio D, Calani L, and Conte A. Antiglycative and antioxidative properties of coffee fractions. Food Chem 124: 1430-1435, 2011.

478. Vitaglione P, Donnarumma G, Napolitano A, Galvano F, Gallo A, Scalfi L, and Fogliano V. Protocatechuic acid is the major human metabolite of cyanidin-glucosides. J Nutr 137: 2043-2048, 2007.

479. Vitaglione P, Sforza S, Galaverna G, Ghidini C, Caporaso N, Vescovi PP, Fogliano V, and Marchelli R. Bioavailability of trans-resveratrol from red wine in humans. Mol Nutr Food Res 49: 495-504, 2005.

480. Walle T, Hsieh F, DeLegge MH, Oatis JE, Jr., and Walle UK. High absorption but very low bioavailability of oral resveratrol in humans. Drug Met Disp 32: 1377-1382, 2004.

481. Wang D, Wei X, Yan X, Jin T, and Ling W. Protocatechuic acid, a metabolite of anthocyanins, inhibits monocyte adhesion and reduces atherosclerosis in apolipoprotein Edeficient mice. J Agric Food Chem 58: 12722-12728, 2010.
482. Wang JF, Schramm DD, Holt RR, Ensunsa JL, Fraga CG, Schmitz HH, and Keen CL. A dose-response effect from chocolate consumption on plasma epicatechin and oxidative damage. J Nutr 130: 2115S-2119S, 2000.

483. Wang LD, Zhou Q, Feng CW, Liu B, Qi YJ, Zhang YR, Gao SS, Fan ZM, Zhou Y, Yang CS, Wei JP, and Zheng S. Intervention and follow-up on human esophageal precancerous lesions in Henan, northern China, a high-incidence area for esophageal cancer. Cancer Chemother 29 Suppl 1: 159-172, 2002.

484. Wang Y, Wang $\mathrm{L}, \mathrm{Wu} \mathrm{J}$, and Cai J. The in vivo synaptic plasticity mechanism of EGb 761-induced enhancement of spatial learning and memory in aged rats. $\mathrm{Br} J$ Pharmacol 148: 147-153, 2006.

485. Wang Z, Fernandez-Seara M, Alsop DC, Liu WC, Flax JF, Benasich AA, and Detre JA. Assessment of functional development in normal infant brain using arterial spin labeled perfusion MRI. Neuroimage 39: 973-978, 2008.

486. Wang-Polagruto JF, Villablanca AC, Polagruto JA, Lee L, Holt RR, Schrader HR, Ensunsa JL, Steinberg FM, Schmitz $\mathrm{HH}$, and Keen CL. Chronic consumption of flavanol-rich cocoa improves endothelial function and decreases vascular cell adhesion molecule in hypercholesterolemic postmenopausal women. J Cardiovasc Pharmacol 47 Suppl 2: S177-S186; discussion S206-S209, 2006.

487. Weisel T, Baum M, Eisenbrand G, Dietrich H, Will F, Stockis JP, Kulling S, Rufer C, Johannes C, and Janzowski C. An anthocyanin/polyphenolic-rich fruit juice reduces oxidative DNA damage and increases glutathione level in healthy probands. Biotechnol J 1: 388-397, 2006.

488. Weseler AR, Ruijters EJ, Drittij-Reijnders MJ, Reesink KD, Haenen GR, and Bast A. Pleiotropic benefit of monomeric and oligomeric flavanols on vascular health-a randomized controlled clinical pilot study. PLoS One 6: e28460, 2011

489. Whelan AP, Sutherland WH, McCormick MP, Yeoman DJ, de Jong SA, and Williams MJ. Effects of white and red wine on endothelial function in subjects with coronary artery disease. Int Med J 34: 224-228, 2004.

490. Widlansky ME, Hamburg NM, Anter E, Holbrook M, Kahn DF, Elliott JG, Keaney JF, Jr., and Vita JA. Acute EGCG supplementation reverses endothelial dysfunction in patients with coronary artery disease. J Am Coll Nutr 26: 95102, 2007.

491. Williams CM, El Mohsen MA, Vauzour D, Rendeiro C, Butler LT, Ellis JA, Whiteman M, and Spencer JP. Blueberry-induced changes in spatial working memory correlate with changes in hippocampal CREB phosphorylation and brain-derived neurotrophic factor (BDNF) levels. Free Radic Biol Med 45: 295-305, 2008.

492. Williams RJ, Spencer JP, and Rice-Evans C. Flavonoids: antioxidants or signalling molecules? Free Radic Biol Med 36: 838-849, 2004.

493. Williamson G and Clifford MN. Colonic metabolites of berry polyphenols: the missing link to biological activity? Br J Nutr 104 Suppl 3: S48-S66, 2010.

494. Willis LM, Shukitt-Hale B, and Joseph JA. Recent advances in berry supplementation and age-related cognitive decline. Curr Opin Clin Nutr Metab Care 12: 91-94, 2009.

495. Winter JC. The effects of an extract of Ginkgo biloba, EGb 761, on cognitive behavior and longevity in the rat. Physiol Behav 63: 425-433, 1998.

496. Winterbone MS, Tribolo S, Needs PW, Kroon PA, and Hughes DA. Physiologically relevant metabolites of quercetin 
have no effect on adhesion molecule or chemokine expression in human vascular smooth muscle cells. Atherosclerosis 202: 431-438, 2009.

497. Wiseman W, Egan JM, Slemmer JE, Shaughnessy KS, Ballem K, Gottschall-Pass KT, and Sweeney MI. Feeding blueberry diets inhibits angiotensin II-converting enzyme (ACE) activity in spontaneously hypertensive stroke-prone rats. Can J Physiol Pharmacol 89: 67-71, 2011.

498. Wong RH, Howe PR, Buckley JD, Coates AM, Kunz I, and Berry NM. Acute resveratrol supplementation improves flow-mediated dilatation in overweight/obese individuals with mildly elevated blood pressure. Nutr Metab Cardiovasc Dis 21: 851-856, 2011.

499. Woods E, Clifford MN, Gibbs M, Hampton S, Arendt J, and Morgan L. Estimation of mean intakes of 14 classes of dietary phenols in a population of male shift workers. Proc Nutr Soc 62: 60A, 2003.

500. Woodward G, Kroon P, Cassidy A, and Kay C. Anthocyanin stability and recovery: implications for the analysis of clinical and experimental samples. J Agric Food Chem 57: 5271-5278, 2009.

501. Wu AH, Yu MC, Tseng CC, and Pike MC. Epidemiology of soy exposures and breast cancer risk. Br J Cancer 98: 9-14, 2008.

502. Wu CH, Lin MC, Wang HC, Yang MY, Jou MJ, and Wang CJ. Rutin inhibits oleic acid induced lipid accumulation via reducing lipogenesis and oxidative stress in hepatocarcinoma cells. J Food Sci 76: T65-T72, 2011.

503. Wu JN, Ho SC, Zhou C, Ling WH, Chen WQ, Wang CL, and Chen YM. Coffee consumption and risk of coronary heart diseases: a meta-analysis of 21 prospective cohort studies. Int J Cardiol 137: 216-225, 2009.

504. Wu X, Cao G, and Prior RL. Absorption and metabolism of anthocyanins in elderly women after consumption of elderberry or blueberry. J Nutr 132: 1865-1871, 2002.

505. Wu X, Pittman HE, McKay S, and Prior RL. Aglycones and sugar moieties alter anthocyanin absorption and metabolism after berry consumption in weanling pigs. J Nutr 135: 2417-2424, 2005.

506. Yang JH, Hsia TC, Kuo HM, Chao PD, Chou CC, Wei YH, and Chung JG. Inhibition of lung cancer cell growth by quercetin glucuronides via G2/M arrest and induction of apoptosis. Drug Metab Dispos 34: 296-304, 2006.

507. Yeh SL, Yeh CL, Chan ST, and Chuang CH. Plasma rich in quercetin metabolites induces G2/M arrest by upregulating PPAR- $\gamma$ expression in human A549 lung cancer cells. Planta Med 77: 992-998, 2011.

508. Yip EC, Chan AS, Pang H, Tam YK, and Wong YH. Protocatechuic acid induces cell death in HepG2 hepatocellular carcinoma cells through a c-Jun N-terminal kinase-dependent mechanism. Cell Biol Toxicol 22: 293-302, 2006.

509. Zern TL, Wood RJ, Greene C, West KL, Liu Y, Aggarwal D, Shachter NS, and Fernandez ML. Grape polyphenols exert a cardioprotective effect in pre- and postmenopausal women by lowering plasma lipids and reducing oxidative stress. J Nutr 135: 1911-1917, 2005.

510. Zhang SM, Hunter DJ, Rosner BA, Giovannucci EL, Colditz GA, Speizer FE, and Willett WC. Intakes of fruits, vegetables, and related nutrients and the risk of non-Hodgkin's lymphoma among women. Cancer Epidemiol Biomarkers Prev 9: 477-485, 2000.

511. Zhu Y, Xia M, Yang Y, Liu F, Li Z, Hao Y, Mi M, Jin T, and Ling W. Purified anthocyanin supplementation improves endothelial function via NO-cGMP activation in hypercholesterolemic individuals. Clin Chem 57: 1524-1533, 2011.
512. Zilkens RR, Burke V, Hodgson JM, Barden A, Beilin LJ, and Puddey IB. Red wine and beer elevate blood pressure in normotensive men. Hypertension 45: 874-879, 2005.

513. Zini A, Del Rio D, Stewart AJ, Mandrioli J, Merelli E, Sola P, Nichelli P, Serafini M, Brighenti F, Edwards CA, and Crozier A. Do flavan-3-ols from green tea reach the human brain. Nutr Neurosci 9: 57-61, 2006.

Address correspondence to: Prof. Alan Crozier Plant Products and Human Nutrition Group School of Medicine University of Glasgow Joseph Black Building Glasgow G12 8QQ United Kingdom

E-mail: alan.crozier@glasgow.ac.uk

Date of first submission to ARS Central, February 24, 2012; date of final revised submission, July 6, 2012; date of acceptance, July 15, 2012.

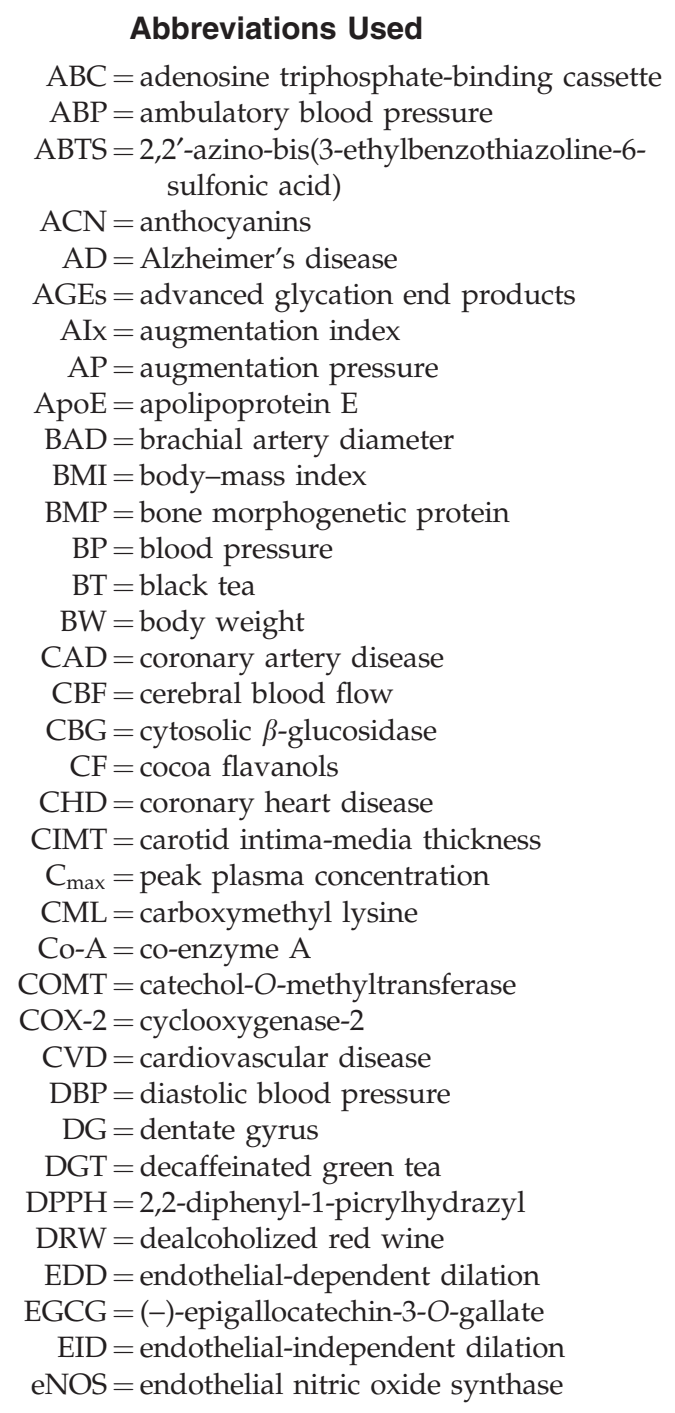




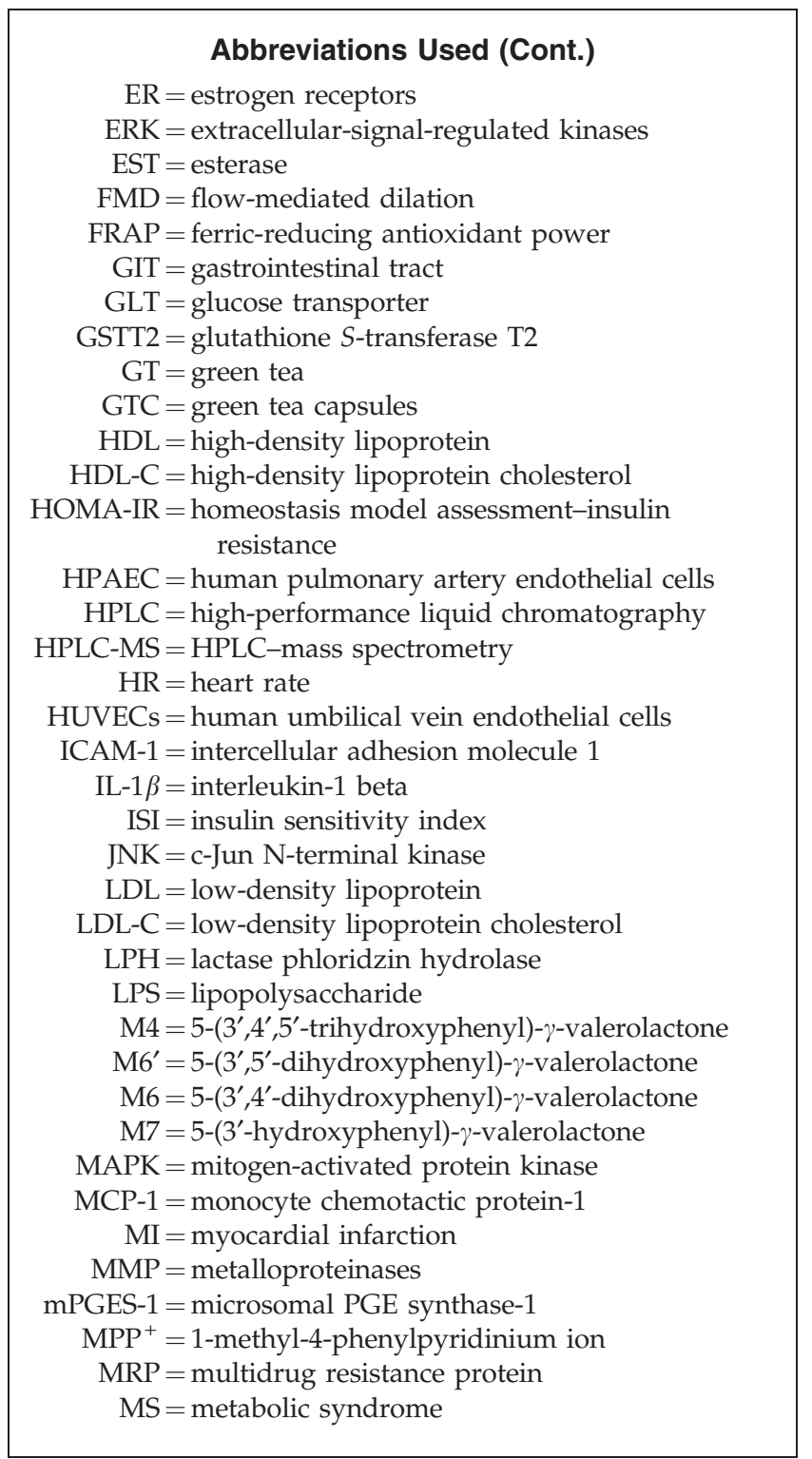

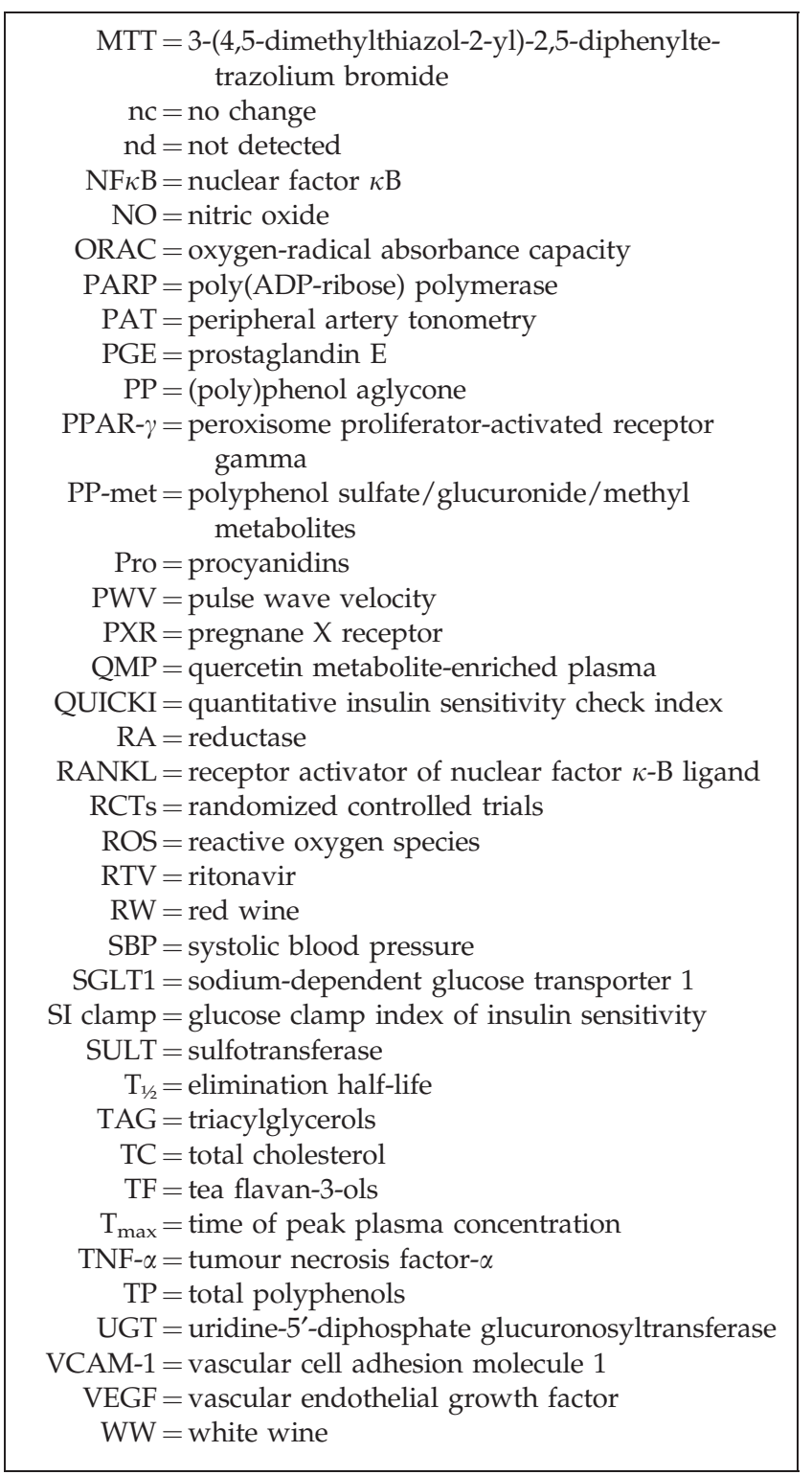

